University of Louisville

ThinkIR: The University of Louisville's Institutional Repository

Electronic Theses and Dissertations

8-2007

\title{
Science inquiry learning environments created by national board certified teachers.
}

Jon Saderholm

University of Louisville

Follow this and additional works at: https://ir.library.louisville.edu/etd

\section{Recommended Citation}

Saderholm, Jon, "Science inquiry learning environments created by national board certified teachers." (2007). Electronic Theses and Dissertations. Paper 1245.

https://doi.org/10.18297/etd/1245

This Doctoral Dissertation is brought to you for free and open access by ThinkIR: The University of Louisville's Institutional Repository. It has been accepted for inclusion in Electronic Theses and Dissertations by an authorized administrator of ThinkIR: The University of Louisville's Institutional Repository. This title appears here courtesy of the author, who has retained all other copyrights. For more information, please contact thinkir@louisville.edu. 


\title{
SCIENCE INQUIRY LEARNING ENVIRONMENTS CREATED BY NATIONAL BOARD CERTIFIED TEACHERS
}

\author{
By \\ Jon Saderholm, NBCT \\ B.A., Berea College, 1987 \\ M.Ed., University of Louisville, 1999

\begin{abstract}
A Dissertation
Submitted to the Faculty of the

Graduate School of the University of Louisville

in Partial Fulfillment of the Requirements

for the Degree of
\end{abstract} \\ Doctor of Philosophy \\ Department of Teaching and Learning \\ University of Louisville \\ Louisville, Kentucky
}

August 2007 
Copyright 2007 by Jon Saderholm

All rights reserved 
SCIENCE INQUIRY LEARNING ENVIRONMENTS CREATED BY NATIONAL BOARD CERTIFIED TEACHERS

\author{
By \\ Jon Saderholm \\ B.A., Berea College, 1987 \\ M.Ed., University of Louisville, 1999 \\ A Dissertation Approved on
}

June 25, 2007

By the following Dissertation Committee:

Dissertation Co-Major Advisor

Dissertation Co-Major Advisor 


\section{DEDICATION}

This work is dedicated to my wife Kimberly around whom my world revolves. 


\section{ACKNOWLEDGEMENTS}

I wish to acknowledge the contribution of the teachers who participated in this study. They did so out of interest in their profession, and willingness to help a fellow teacher. I also wish to acknowledge the work of my wife Kimberly, and my sons Benjamin and Ian, who worked long hours processing the data for this study. Lastly, I would like to acknowledge my advisors, Tom Tretter and Robert Ronau whose broad perspectives and kind input enabled me to conceive of this project and see it to fruition. 


\title{
ABSTRACT \\ SCIENCE INQUIRY LEARNING ENVIRONMENTS CREATED BY NATIONAL BOARD CERTIFIED TEACHERS
}

\author{
Jon Saderholm
}

June 25. 2007

The purpose of this study was to discern what differences exist between the science inquiry learning environments created by National Board Certified Teachers (NBCTs) and non-NBCTs. Four research questions organized the data collection and analysis: (a) How do National Board Certified science teachers' knowledge of the nature of science differ from that of their non-NBCT counterparts? (b) How do the frequencies of student science inquiry behaviors supported by in middle/secondary learning environments created by NBCTs differ from those created by their non-NBCT counterparts? (c) What is the relationship between the frequency of students' science inquiry behaviors and their science reasoning and understanding of the nature of science? (d) What is the impact of teacher perceptions factors impacting curriculum and limiting inquiry on the existence of inquiry learning environments?

The setting in which this study was conducted was middle and high schools in Kentucky during the period between October 2006 and January 2007. The population sampled for the study was middle and secondary science teachers certified to teach in Kentucky. Of importance among those were the approximately 70 National Board 
Certified middle and high school science teachers. The teacher sample consisted of 50 teachers, of whom 19 were NBCTs and 31 were non-NBCTs.

This study compared the science inquiry teaching environments created by NBCTs and non-NBCTs along with their consequent effect on the science reasoning and nature of science (NOS) understanding of their students. In addition, it examined the relationship with these science inquiry environments of other teacher characteristics along with teacher perception of factors influencing curriculum and factors limiting inquiry.

This study used a multi-level mixed methodology study incorporating both quantitative and qualitative measures of both teachers and their students. It was a quasiexperimental design using non-random assignment of participants to treatment and control groups and dependent pre- and post-tests (Shadish, Cook, \& Campbell, 2002). Teacher and student NOS understanding was measured using the Student Understanding of Science and Science Inquiry (SUSSI) instrument (Liang, et. al, 2006). Science inquiry environment was measured with the Elementary Science Inquiry Survey (ESIS) (Dunbar, 2002) which was given both to teachers and their students. Science inquiry environment measurements were triangulated with observations of a stratified random sub-sample of participating teachers. Observations were structured using the low-inference Collaboratives for Excellence in Teaching Practice (CETP) Classroom Observation Protocol (COP) (Lawrenz, Huffman, \& Appleldoorn 2002), and the high-inference Reform Teaching Observation Protocol (RTOP) (Piburn \& Sawada, 2000).

NBCTs possessed more informed view of NOS than did non-NBCTs. Additionally, high school science teachers possessed more informed views regarding 
NOS than did middle school science teachers, with the most informed views belonging to high school science NBCTs. High school science NBCTs created learning environments in which students engaged in science inquiry behaviors significantly more frequently than did high school science non-NBCTs. Middle school science NBCTs, on the other hand, did not create learning environments that differed in significant ways from those of middle school science non-NBCTs. Students of high school science NBCTs possessed significantly higher science reasoning than did students of high school science nonNBCTs. Middle school students of science NBCTs possessed no more science reasoning ability than did middle school students of science non-NBCTs. NOS understanding displayed by students of both middle school and high school science NBCTs was not distinguished from students of non-NBCTs.

Classroom science inquiry environment created by non-NBCTs were correlated with science teachers' perceptions of factors determining the curriculum, and the factors limiting inquiry. NBCT classroom science inquiry environment were not correlated with science teacher perceptions. They were, however, strongly correlated with science teacher attendance at science workshops and negatively correlated with teacher perception that experience limits inquiry.

The results of this study have implications for policy, practice, and research. Having a science teacher who is an NBCT appears to benefit high school students; however, the benefit for students of middle school science NBCTs appears only when the teacher is also experienced. Additionally, science NBCTs appear to be able to create more controlled science inquiry learning environments than do science non-NBCTs. At the high school level the practice of using data to explain patterns appears to positively 
affect student science reasoning. Implications results of this study have for further research include examining the differences of the NBPTS certification process for middle and high school teachers; deeper investigation of the causes of the differences in science reasoning between students of NBCTs and non-NBCTs; and studies of the relationship between the NBPTS certification process and teacher efficacy and personal agency. 


\section{TABLE OF CONTENTS}

LIST OF TABLES ............................................................. xviii

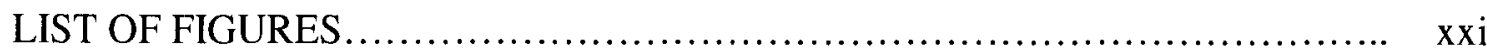

CHAPTER 1-INTRODUCTION............................................... 1

CHAPTER 2-REVIEW OF LITERATURE .................................. 8

Introduction....................................................... $\quad 8$

Nature of Science........................................................

The Structure of Scientific Knowledge............................ $\quad 9$

The Nature of the Scientific Enterprise.......................... 10

Science in tentative...................................... 11

Science is an antagonistic endeavor....................... 11

Science is not a normal activity........................... 12

Science is not extended common sense .................... 14

Science is a social action.................................... 15

Conclusion.............................................. 17

Nature of Science Instruction........................................ 17

Constructivism as the Referent for Science Pedagogy............... 17

Psychological Constructivism.................................. 18

Social Constructivism....................................... 20

The Special Relationship Between Constructivism and Science....... 21 
The History of Science Instruction in the U.S. ........................... 22

The National Call for Science Inquiry Instruction........................ 25

Description of Science Inquiry Instruction................................ 27

Practice Described in National Standards......................... $\quad 27$

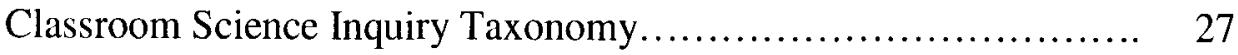

Science Inquiry Learning Environments........................ 29

Factors Impacting Science Inquiry Instruction.................. 30

Current State of Science Inquiry Instruction....................... 33

Impacting Inquiry Abilities in the Classroom............................ 34

Assessing Students' Inquiry Knowledge and Ability............... 34

Impact on Inquiry-Related Skills............................... 34

Impact on Content Knowledge............................... 35

Relationships with Teacher Characteristics....................... 36

Teacher Efficacy and Personal Agency................................. $\quad 37$

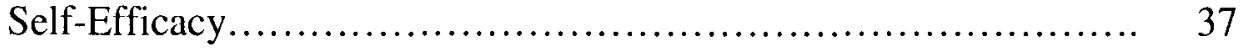

Personal Agency ............................................ 38

The National Board for Professional Teaching Standards.................. 39

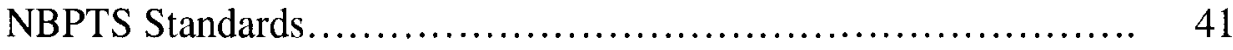

NBPTS Science Portfolio..................................... 42

National Board Certification Correlation with Teacher Quality....... 43

National Board Certification as Effective Professional Development. 47

Literature Review Conclusion....................................... 48

CHAPTER 3-METHODOLOGY .......................................... 50 
Rationale.............................................................. 50

Overview of Methodology ........................................... 52

Science Reasoning and NOS Understanding Operational Definitions. $\quad 53$

Study Design.................................................. 54

Data Collection Schedule...................................... $\quad 55$

Study Population.............................................. 57

NBCTs............................................... 57

Non-NBCTs......................................... 57

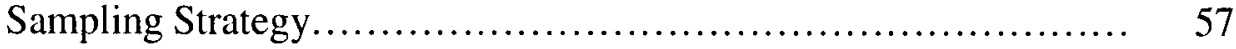

Solicitation of teacher participants....................... 58

Selection of student participants......................... $\quad 60$

Participants.................................................... 60

Description of teacher sample............................ $\quad 60$

Description of student sample........................... $\quad 65$

Summary................................................. 67

Variables and Measures............................................. 67

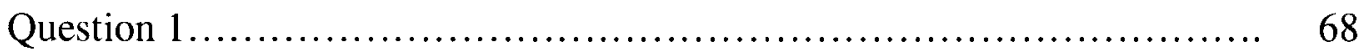

Instrumentation................................................. 68

Analysis.................................................... 70

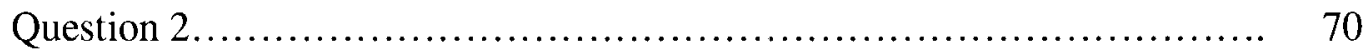

Instrumentation.............................................. 71

Observation Protocols......................................... 72

Establishing Instrument Validity................................ $\quad 74$ 
Correlation between teacher and student ESIS responses..... 74

Internal consistency reliability......................... 75

Comparison of teacher and student ESIS response trends..... 77

Factor analysis..................................... 78

Analysis........................................................ 79

MANOVA............................................. 79

Observation........................................... 79

Documentary analysis................................. 80

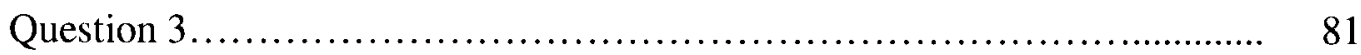

Variables.................................................... 81

Independent variables................................ 81

Dependent variables................................ 81

Instrumentation............................................... 82

Analysis................................................. 83

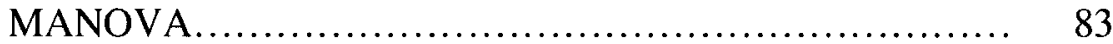

Regression.............................................. 84

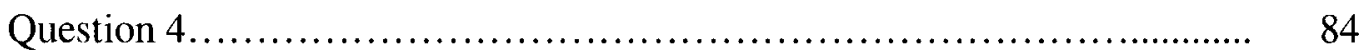

Classroom Level Variables....................................... 86

Analysis...................................................... 86

Correlation.............................................. 86

Validity ............................................................ 87

Construct Validity........................................ 87 
External Validity

Statistical Conclusion Validity .................................... 88

Effect size............................................... 88

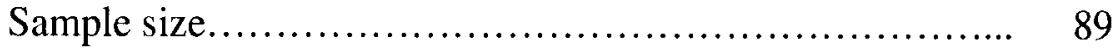

Self-report bias........................................... 89

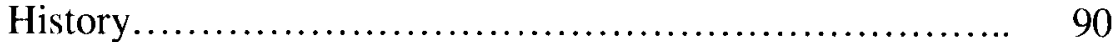

Maturation............................................. 90

Limitations.............................................................. 91

Inferences Regarding Teachers and Teacher Practice............... 91

Inferences Regarding the Effect of Teacher Characteristics and Practice on Student Achievement................................. 93

CHAPTER 4-RESULTS.......................................................... 95

Introduction........................................................... 95

Question 1 Results............................................ 95

NOS Understanding Comparison.................................... 95

NBCT status............................................ 97

Middle/high school status.................................... $\quad 99$

Non-education degree................................. 99

Certification rank...................................... 100

NBCT status-middle/high school interaction............... 100

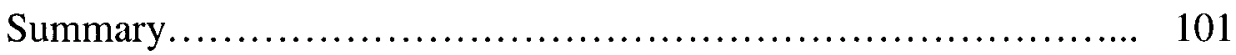

Significant results..................................... 101

Non-significant results............................... 102 
Overall.

Analysis of Classroom Inquiry Behaviors

Other Classroom Pedagogical Practices.

Classroom Observation

Susan

Teacher and school description.

Observation description and analysis

Document analysis

Audrey

Teacher and school description 112

Observation description and analysis

Document analysis

Robert

Teacher and school description.

Observation description and analysis

Document analysis

Rhonda. 116

Teacher and school description.......................... 116

Observation description and analysis

Document analysis.

Jackie. 
Teacher and school description.

Observation description and analysis................... 118

Document analysis.................................. 119

Kyle...................................................... 119

Teacher and school description........................... 119

Observation description and analysis.................... 119

Document analysis.................................. 120

Jennie................................................... 120

Teacher and school description........................ 120

Observation description and analysis................... 121

Document analysis................................. 121

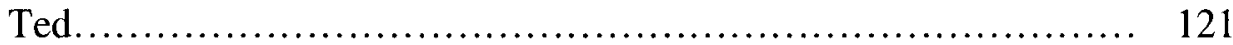

Teacher and school description........................ 122

Observation description and analysis.................... 122

Document analysis................................. 122

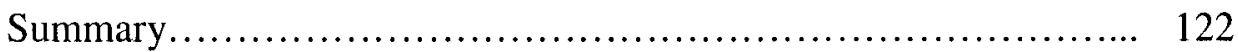

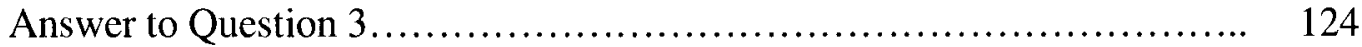

Differences in Student Science Reasoning and NOS Understanding. 124

Relationship of Student Science Reasoning and NOS Understanding with Teacher Variables................................... 127

High school student achievement and teacher variables...... 128

Middle school student achievement and teacher variables..... 128

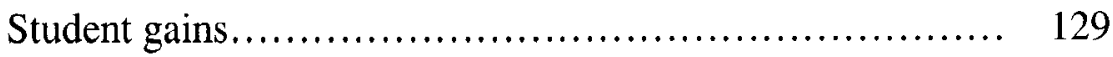


Differences in Teacher Practice................................. 130

Students of middle school non-NBCTs.................... 130

Students of high school non-NBCTs...................... 132

Students of middle school NBCTs....................... 133

Students of high school NBCTs........................... 134

Summary.................................................... 134

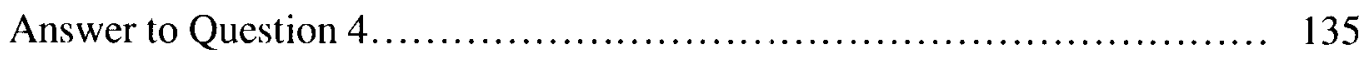

Middle and High School Groups............................... 135

Teacher characteristics................................. 135

Teaching context.................................... 137

NBCTs and non-NBCTs................................. 140

Teacher characteristics............................... 140

Teaching context................................... 142

Results Summary............................................... 145

CHAPTER 5-DISCUSSION AND IMPLICATIONS ........................ 147

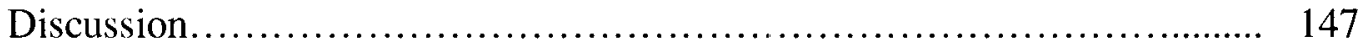

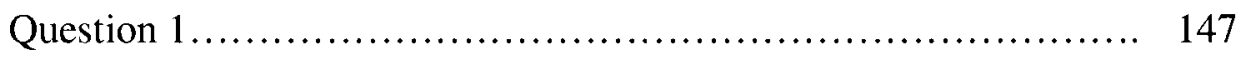

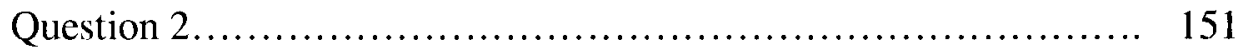

Question 3.............................................. 155

Student science reasoning ............................. 156

Student NOS understanding............................ 158

Differences were noted on first phase of assessment......... 159

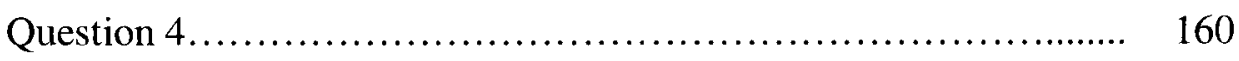


Implications.

Implications for classroom practice............................... 163

Implications for future research............................... 164

Implications for policy..................................... 165

Implications for teacher education............................ 166

Implications for the NBPTS certification process............... 166

Conclusion............................................................ 167

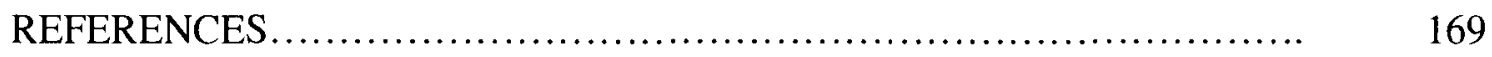

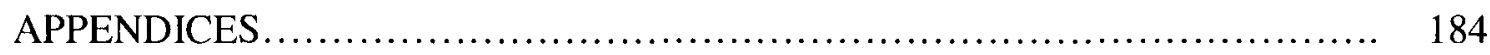

Appendix A: Research Question—Data Source Matrix................... 185

Appendix B: ESIS Questionnaire.................................... 186

Appendix C: SUSSI Assessment................................... 190

Appendix D: CETP Observation Protocol............................... 193

Appendix E: RTOP Observation Protocol................................ 194

Appendix F: Course Information................................. 199

Appendices G1-G6: Course Documentation........................ 202

CURRICULUM VITAE........................................... 229 


\section{LIST OF TABLES}

Table 3.1 Phases of Data Collection........................................ 56

Table 3.2 Teacher Demographics Describing First and Second Phase Samples....... 61

Table 3.3 Teacher non-Education College Degrees............................. 62

Table 3.4 Teacher Licensure Level....................................... 63

Table 3.5 Description of Years Experience for Teacher Sample by Experience Level.. 65

Table 3.6 Number of Students by Grade Level................................... 65

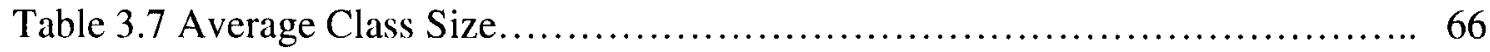

Table 3.8 Teacher Perceptions of Participating Class' Achievement................ 66

Table 3.9 School-level Demographic Variables............................. 66

Table 3.10 Teacher/Student ESIS Correlations by Grade, NBCT Status, and 75 Experience......................................................

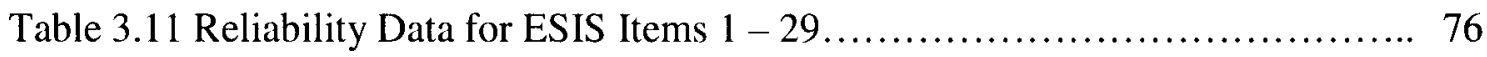

Table 3.12 ESIS Item Factor Assignment............................... 76

Table 3.13 LTSR Question-Construct Assignment........................... 82

Table 3.14 Teacher Background and Context Measures........................ 85

Table 4.1 Means and (Standard Deviations) of Teachers' SUSSI Scores Across Five Dimensions of Teacher Categorization.................................. 97

Table 4.2 ESIS Teacher Behavior Variable Assignment........................ 103

Table 4.3 ESIS Results for non-NBCT and NBCT Groups..................... 104

Table 4.4 Multivariate and Univariate Analysis of Variance F Ratios for High School NBCT, Non-Education Degree, and Experience Level on Inquiry Environment 106 
Factors

Table 4.5 Multivariate and Univariate Analysis of Variance F Ratios for Middle

School NBCT, Non-Education Degree, and Experience Level on Inquiry

Environment Factors.

Table 4.6 Teacher General Pedagogical Practice Variables

Table 4.7 Characteristics of Observed Teachers 108

Table 4.8 Means and Standard Deviations of Students' Science Reasoning and NOS

Understanding Scores.

Table 4.9 One-Way Analyses of Variance of Effects of Student Assignment on

Phase-2 Science Reasoning and NOS Understanding.

Table 4.10 Multivariate and Univariate Analyses of Variance F Ratios for High

School NBCT Status, Degree and Experience Effects on Student Science

Reasoning and NOS Understanding.

Table 4.11 Multivariate and Univariate Analyses of Variance F Ratios for Middle

School NBCT Status, Degree and Experience Effects on Student Science

Reasoning and NOS Understanding.

Table 4.12 Simultaneous Regression of Science Reasoning on Inquiry Environment Factors and Students' Grade in School.

Table 4.13 Middle School Teacher Characteristics Correlations with Classroom Inquiry Practices

Table 4.14 High School Teacher Characteristics Correlations with Classroom Inquiry Practices.

Table 4.15 Middle School Teaching Contexts Correlations with Classroom Inquiry

Practice.

Table 4.16 High School Teaching Contexts Correlations with Classroom Inquiry

Practice.

Table 4.17 Teacher Constructed Responses to the "Other" Category.

Table 4.18 Non-NBCT Characteristics Correlations with Classroom Inquiry Practice.. 141

Table 4.19 NBCT Characteristics Correlations with Classroom Inquiry Practice. 142

Table 4.20 Non-NBCT Teaching Context Correlations with Classroom Inquiry 
Practice.

Table 4.21 NBCT Teaching Context Correlations with Classroom Inquiry Practice.....144 


\section{LIST OF FIGURES}

Figure 2.1 Spectrum of Classroom Inquiry Activities............................ 29

Figure 3.1 Quasi-Experimental Study Design..................................... 54

Figure 3.2 Phases of Data Collection and Analysis.............................. 58

Figure 3.3 Experience of Second Phase Teacher Sample Paneled by NBCT Status and Experience Level..................................................... 64

Figure 3.4 Comparison of Mean Teacher and Student Responses to ESIS Classroom Inquiry Questions............................................................ 77

Figure 4.1 Graph of Student Science Reasoning Score Means from Phase-1 and Phase-2 of Data Collection.

Figure 4.2 Graph of Student NOS Understanding Score Means from Phase-1 and Phase-2 of Data Collection. 


\section{INTRODUCTION}

"Children should be led to make their own investigations, and to draw their own inferences. They should be told as little as possible, and induced to discover as much as possible."-Herbert Spencer, 1864, p. 124-125.

"The call for students to be familiar with the methods of science inquiry and reasoning and to understand the concepts and processes of the sciences remains a visible, but largely unmet, national educational goal." -America's Lab Report, 2006, p. vii.

Science is not merely a body of knowledge describing the natural world, but a practice that creates knowledge of the natural world. Subsequently, in order for science teachers to be able to claim success in their endeavor, they must not only teach students the knowledge created by science, but also how scientific knowledge is created. Without the structure provided by the skills of science reasoning and the dispositions taught by understanding the nature of science, the content of science becomes an endless litany of facts indistinguishable from those of any other domain. Furthermore, the body of knowledge describing the natural world is so large and evolving so rapidly it is simply incorrect to say a student who learned the set of facts describing science as it existed during his schooling has "learned science." Much of the science instruction occurring in the U.S. today teaches the facts of science without reference to the intellectual and social 
processes that created them. Fortunately however, a spectrum of teaching practices is present in U.S. classrooms. Represented among these practices are those of accomplished teachers who do provide students access to the full intellectual, social, and factual experience of science. The purpose of this project is to identify these effective teachers and analyze their practice.

A review of the history of science education in the United States shows that educational goals and pedagogical techniques have evolved as psychological understanding developed, responding to societal imperatives as the twentieth century unfolded (DeBoer, 1991). However, as illustrated by the two quotes at the beginning, throughout the 150 years between the Civil War and the turn of the millennium, the call for students to learn scientific inquiry skills and habits of thought continued to be broadcast unchanged and unanswered, even during the modern era (DeBoer, 1991). Science for All Americans (American Association for the Advancement of Science (AAAS), 1990) is nearly twenty years old and yet, in large part, $21^{\text {st }}$ century science classrooms and scholastic environments still resemble those that existed when it was published. Current practice in science classrooms does not emphasize science inquiry, or indeed, deep understanding of schematic science knowledge (Marlow, \& Stevens, 1999; Ruiz-Primo, Li \& Shavelson, 2002). The relative stability of science teaching practice contributing to this state of affairs may be due to a confluence of institutional and teacher qualities.

Upon first analysis, one might understand this institutional inertia to be related to the high stakes testing environment characterizing many schools, or to funding and staffing levels that are not conducive to the creation of classroom inquiry environments- 
and no doubt these factors do impact the state of science inquiry practice in U.S. classrooms. On a deeper level however, this resistance may be traced to more foundational issues. The pragmatic teacher philosophy motivating practice has emphasized teacher control of a large group of passive learners. Schools have been described as organizations that do not have consensus on their desired product, cannot directly observe the process of production, and have difficulty describing how they make their products. Organizations such as this are best described as coping organizations (Cuban, 1995). It should not be surprising that such organizations resist reform.

If changes in this aspect of science instruction are to be systematically enacted, looking to institutions so described for their genesis is unreasonable. Instead, looking for those teachers who are able to create effective science inquiry environments within current institutional contexts and studying how these teachers navigate and mediate existing institutional characteristics to do so would be a much more effective strategy. Because it is improbable that public school structures will quickly change in a way that encourages science teachers to enact science inquiry instruction, modeling innovations based upon the experience of successful practitioners may lead more directly to the recommended evolution in U.S. science teaching.

Managing active science inquiry instruction requires a challenging set of teacher habits and skills. Teachers must be able to create environments in which students actively pursue the answers to questions using scientific reasoning and methods (NRC, 2000). Because it requires the maintenance of dynamic equilibrium between competing and disparate forces, science inquiry is among the most difficult forms of science instruction to enact (Baker, Lang, \& Lawson, 2002). These inquiry environments are constructivist 
in nature, require a reconceptualization of textbook resources, and require both teacher and student to assume new roles (Anderson, 2002). Teaching science as inquiry is challenged by teacher beliefs (Wallace, 2004; Roehrig \& Luft, 2004), along with parent, student, and administrator dispositions (Wenning, 2005a). In general, teachers lack the necessary science inquiry instruction skills, and institutional characteristics and administrative policies prevent teachers from gaining them (NRC, 2006).

Any study of teacher practice or student learning must have a theory of learning as its foundation. This study is grounded in the paradigm that individuals construct the understandings they possess. This paradigm is taken to be true whether understanding is constructed from the unstructured social or physical environment, or from the intentional learning environment created in a classroom. To view the act of teaching as simply the delivery of knowledge is to miss its essential quality, casting students in the form of automata whose unique perspectives and abilities to make choices may be ignored. Constructivist approaches to teaching are informed by the recognition of the primacy of students' perspectives and their capacity to make choices. This interpretation helps define the role of a teacher for this study.

Classrooms are considered to be learning environments dynamically controlled by teachers in which students construct knowledge. The extent to which teachers are able to conceptualize the content to be learned, organize and manage the learning sequence, anticipate students' cognitive and affective orientations, and manage the activity in the classroom influences the success of students learning the material. Effective science teachers are comfortable in these fluid environments encouraging students to reason scientifically and engage in activity emulating the practice of science. 
A pool of accomplished science teachers has been identified that may serve as a source of effective implementation of inquiry-based teaching which may server to inform the practice of other science teachers attempting to create science inquiry environments in their classrooms. The National Board for Professional Teaching Standards (NBPTS) developed standards of accomplished practice for science instruction (NBPTS, 2003a, 2003b). The first Adolescence and Young Adult Science (AYA Science) certificates were issued in 1998, and the first Early Adolescence Science (EA Science) certificates were issued in 1999. To earn this certification, teachers must prepare a portfolio documenting, among other things, their ability to enact active science inquiry instruction. Evidence for this portfolio is developed from classroom instruction and requires submission of documentary and videotape evidence (NBPTS, 2004a, 2004b). The NBPTS estimates that the entire certification attempt requires some 200 to 400 hours of concentrated effort from candidates (NBPTS, 2004a).

The NBPTS certification process has been shown to identify accomplished teaching practice and those teachers who perform to this rigorous set of standards. National Board Certification has been shown to effectively distinguish accomplished teachers from among the elementary school teacher population. Bond, Smith, Baker, and Hattie (2000) identified 13 categories of accomplished teaching. NBCTs outperformed their non-certified counterparts on 11 of the 13 categories. NBCTs also have been shown to utilize assessment more effectively (O'Sullivan, 2005). More recently, the NBPTS certification process was shown to improve science teachers' inquiry and assessment abilities (Lustick \& Sykes, 2006). 
Given the calls by national science education organizations (AAAS, 1994; NRC, 1996) for increased science inquiry instruction and student initiative in the design of questions and experiments, and in light of the evidence that supportive inquiry learning environments are not common in American schools, it is important to study the practices of those teachers who are able to navigate the personal and institutional obstacles to enact effective science inquiry instruction. The contrast of the explicit nature of the NBPTS active science inquiry expectations with the evidence that science teachers as a whole do not teach using the inquiry model (Ruiz-Primo, Li \& Shavelson, 2002), indicates that differences may be found between the science inquiry pedagogical practice of NBCTs and their non-NBCT counterparts. Comparing the practices of these accomplished teachers against those of their counterparts may shed light on teacher characteristics and behaviors that more effectively promote active science inquiry among students.

In spite of the breadth and depth of studies describing science inquiry instruction and learning, this study is well situated to contribute to science education research because little research has been conducted to date investigating the practice and impact of middle and secondary National Board Certified science teachers. No studies to date have investigated the science inquiry pedagogical practice of middle and high school science NBCTs. The majority of research on effect of NBPTS certification on student achievement has concerned elementary school children. Only two studies have examined the impact of the NBPTS certification process on science teachers (Lustick, 2002; Lustick \& Sykes, 2006), but they did not directly examine the classroom practice of NBCTs or its impact on students. 


\section{Research Questions}

This study explores the science inquiry pedagogical practices and nature of science understanding of accomplished middle and high school science teachers as they construct science inquiry learning environments. Furthermore, the study attempts to discern what, if any, effect these environments have on middle and high school students' scientific reasoning and nature of science comprehension. Specifically, this study attempts to answer the following questions:

1. How do National Board Certified science teachers' knowledge of the nature of science differ from that of their non-NBCT counterparts?

2. How do the frequencies of student science inquiry behaviors supported by in middle/secondary learning environments created by NBCTs differ from those created by their non-NBCT counterparts?

3. What is the relationship between the frequency of students' science inquiry behaviors and their science reasoning and understanding of the nature of science?

4. What is the impact of teacher perceptions factors impacting curriculum and limiting inquiry on the existence of inquiry learning environments? 


\section{LITERATURE REVIIEW}

\section{Introduction}

The research hypothesis tested by this study was that National Board Certified Teachers (NBCTs) create learning environments that positively impact students' science reasoning skills and understanding of the nature of science (NOS) in measurably different ways from non-NBCTs. An assumption grounding this research was that teachers may change students' science reasoning and NOS conceptions. This chapter describes both science reasoning and NOS and provides supporting documentation that both may be affected by instruction.

The epistemological paradigms situating this study are psychological and social constructivism. Teacher classroom behaviors were considered to be associated with student outcomes through the creation and management of social learning environments in which students psychologically construct knowledge. Since these paradigms are related to the way science is practiced and the way its knowledge is accumulated, this chapter will explicate their connection.

Science classrooms do not exist in a vacuum, but rather are positioned within a rich history of U.S. education and situated under the microscope of public policy. In order to discern qualities of practice that distinguish one group of teachers from another, understanding the current state of instruction, its historical context, and current policy environment is important. To this end, the history of science instruction relating to 
inquiry pedagogy in particular is described, as well as current standards and policy recommendations.

This study compared groups of middle and secondary science teachers characterized by professional and demographic factors. The relationships of these factors with teaching practices is established. Of importance to the instructional decisions teachers make is their sense of self efficacy and personal agency. The impact of expertise is important as well. In particular, a description of the formation of the National Board for Professional Teaching Standards (NBPTS) is provided, as is a description of the National Board Certification process, and evidence of its correlation with accomplished teaching.

\section{Nature of Science}

\section{The Structure of Scientific Knowledge}

The body of knowledge of the natural world characterized as scientific knowledge is different from other forms of knowledge in several important ways. Chief among them is the absolute authority granted to observable reality. This is documented in an account drawn from the field of astronomy. In 1992, astronomer Andrew Lyne was on the verge of announcing his discovery of the first extra-solar planet. A few days before the international meeting at which he was scheduled to speak, he found that his "discovery" was actually the result of not properly accounting for the orbit of the Earth around the sun in his calculations. Anticipating embarrassment and shame, he announced his mistake at his presentation in front of hundreds of prominent astronomers. Instead, the assembled scientists responded with an extended ovation (Seife, 2000). In that moment, those scientists were celebrating the process of science. One of their members felt so strongly 
about the veracity of the scientific process that he was willing to risk his career in its pursuit. The willingness to toss out conclusions in the face of new evidence is precisely (and ironically) what makes scientific theories so strong.

Observations themselves do not explain physical reality. Meaning is attached to observations primarily by the scientific paradigm under which the scientist operates. A set of measurements seen as meaningless when viewed from one perspective may be interpreted as a prime exemplar of a theory when viewed from a different perspective. In the Structure of Scientific Revolutions, Kuhn (1970) described instances of this in which data already in the hands of researchers remained meaningless until a shift in "reality" could enable their explanation. His examples included the Copernican revolution, Lavoisier's discovery of oxygen, and Einstein's theory of relativity. Kuhn's (1970) conception was that the evolution of scientific knowledge proceeds by the slow accumulation of evidence supporting a theoretical structure, punctuated by revolutions in the paradigm through which that evidence is interpreted.

\section{The Nature of the Scientific Enterprise}

Much has been written about the nature of science and the qualities of scientific inquiry that distinguish science from other forms of study (e.g. AAAS, 1990; Kuhn, 1996; Lederman, 1992; Popper, 1968; Russell, 1914; Schwab, 1962a). Science, like other modes of inquiry, attempts to make meaning of the world; however, science differs from other meaning-making pursuits in important ways. The purpose of science is "the acquisition of objective knowledge concerning the structure and behavior of the physical universe" (Hubble, 1954, p. 6). Science is differentiated from other intellectual pursuits through its use of induction and falsification (Popper, 1968). Current research and 
national science education reform documents have identified several important aspects of the nature of science (Lederman, 1999). Scientific knowledge is tentative. It is simultaneously empirically based and subjective. Science requires a combination of objective observations while involving human creativity to draw inferences. Lastly, science is socially embedded (Lederman, 1999). A thorough discussion of the nature of science is a critical component of the foundation of this study.

Science is tentative. At its core, this philosophical statement reflects scientists' recognition that they cannot know or observe all events. This is a result of the primacy of observation. Perfect scientific explanations are impossible because all observations cannot be made (AAAS, 1990). Scientific hypotheses must account for all pertinent observations. A necessary consequence of this requirement is that science is progressive (Popper, 1962), meaning it always moves forward because each new theory must account for previous theories as it predicts new undiscovered laws (Hubble, 1954). In recognition of the necessarily limited perspective of scientists, all scientific theories are constructed to be falsifiable (Popper, 1962). Thus every scientific hypothesis must be designed to incorporate the tools of its own demise since it must be falsifiable in order to guarantee an appeal to the natural world.

Science is an antagonistic endeavor. A consequence of the tentative nature of scientific hypotheses is that the scientific enterprise is typified by attempts to disprove or modify existing hypotheses and theories. For this reason, science is antagonistic in nature-setting the ideas of one practitioner against those of another, or even against oneself (Popper, 1962). This process ensures that at whatever stage a line of inquiry 
exists, the currently accepted theory most effectively explains the observations (Wolpert, 1993).

Science is not a normal activity. A common conception is that abstract reasoning is an ability that develops innately in humans in the absence of intentional instruction, given sufficient psychological development (Wolpert, 1993). An inference might be drawn that the advanced scientific and technological state to which industrialized societies have developed is a natural stage for any culture, given sufficient time and resources. This inference relies on the assumption, however, that the possession of scientific reasoning ability is grounded solely in abstract reasoning capacity and that cultural factors are not also influential. The capacity to reason abstractly does not automatically lead to the ability to do so. In fact, historical analysis shows that in all instances of cultures that developed abstract reasoning, the development of these skills may be traced to a single ancient Greek origin (Cromer, 1993). If environmental and cultural factors do indeed affect the nature and extent of the intellectual skills humans develop, then the possession of scientific habits of mind is seen to be constrained by cultural identity (Diamond, 1999). The capacity to reason abstractly is a necessary but not sufficient condition for the actual development of the abstract thinking necessary for scientific reasoning (Cromer, 1993).

Throughout their evolution, humans have been making meaning of the natural world. Early hunter-gatherers may have negotiated their world using simple superstitions and cultural mores to inform their behavior. As culture evolved and writing and mathematical skills developed, natural patterns and cycles could be discerned and recorded. The perspective gained by these skills enabled these cultures to predict seasonal 
and meteorological events. For the majority of human history this perspective simply augmented the existing mythic and superstitious explanations of the natural world. Many cultures, including the Babylonian and Indian cultures for example, reached this level of sophistication only to remain there for millennia, not gaining the skills of logical abstraction and scientific methodology (Cromer, 1993).

In Uncommon Sense: The Heretical Nature of Science, Cromer (1993) argued that the conditions necessary for the birth of scientific habits of mind are not a naturally occurring aspect of culture, but are aberrations. He argued that abstract thought only germinated once in human history in ancient Greece, and all other instances where it exists could be traced to this seminal event. As part of Cromer's evidence, he documented the thousands of years that advanced civilizations existed without developing abstract algebra or logical proof. Only the ancient Greek culture spontaneously generated these concepts, from which evolved scientific rationality that is the foundation of our Western world-view today.

The argument that the ability to reason abstractly is an aberration and not a necessary condition of advanced culture focuses attention on those processes existing today that either enhance or hinder hypothetico-deductive reasoning in our culture. If the habits of thought that are hallmarks of our technological culture are not naturally occurring, then they must be brought into existence and nurtured in a systematic, intentional way. If Cromer's (1995) argument is valid, then the role of the teacher is exceptionally important in the development of scientific habits of thought for the next generation. 
Science is not extended common sense. A perspective held by distinguished thinkers including Thomas Huxley and Alfred North Whitehead was that science as a meaning-making activity is nothing more than systematized common sense (Wolpert, 1993). However, philosophy of science literature describes scientific thought as quite different from common sense. Examination of the gedanken experiments Galileo, Newton, and Einstein performed in their analyses of simple states of motion highlights that "science often explains the familiar in terms of the unfamiliar" (Wolpert, 1993, p. 3). Popper (1962) argued that experience yields knowledge of singular observations, not universal principals. Science is the inductive process through which individual observations are synthesized and extended, enabling the prediction of as-yet-unseen phenomena. For instance, the instruction universally presented in physics classes to "just ignore friction for a minute" is a request for students to consider concepts that are-at least in the present-beyond their capacity to directly observe, and therefore beyond the reach of common sense. Mendeleyev's synthesis of many individual observations to create a periodic table of elements and subsequently use it to predict the existence of new, undiscovered elements is an example of this process.

The application of scientific and logical thought enables the human mind to encompass concepts of greater expanses of distance and time than human senses alone are able to perceive. The proof of the irrationality of $\pi$, the wave/particle duality of light, and extent of geologic history are examples of this. But the enhanced perception that logical thought enables does not automatically occur as a consequence of human development. For instance, the ability to conceptualize scale correctly and effectively has been shown not only to be related to age (development), ability (academic status), but 
also to training (expertise) (Tretter, Jones, Andre, Negishi, \& Minogue, 2006). The cognitive skills necessary to "do science" are developmentally linked, meaning that younger children cannot perform certain science tasks regardless of their training (Lawson, 1978). However, developmental stage is clearly only a necessary but not sufficient condition for facility with science (Lawson, 1984). Development of these skills is contingent on experience and training as well.

Science is a social action. Science is not done by lone scientists in isolation, but by complex networks of scientists influenced and informed by their social circumstance (AAAS, 1990). Kuhn (1962) argued that the social and historical context in which science is conducted influences the explanations created by scientists to explain observations. Since then, discussions about the sociology of science have caused a reevaluation of the rationality of scientists in light of the perspective that scientists' judgments are socially contingent (Whitley, 2000). This contingency may originate subtly in the way individuals construct meaning from language. It may also take explicit form in the manipulation or suppression of scientific results due to social or political contexts. Evaluation of the nature and progress of science in this light alters the perspective that science is a natural and purely rational mode of investigation.

Modern science may be described as a reputational work organization (Whitley, 2000). This is to say scientists do their work for social and economic reasons as well as for its intrinsic enjoyment (AAAS, 1990). The solitary scientist practicing alone in his laboratory is becoming less and less characteristic of the image of science held by many in the public. With the recognition of the economic and political benefits bestowed by science, nations and corporations have increasingly become engaged in the support and 
production of scientific research by introducing social, political, and economic dimensions into the research process (Whitley, 2000).

Examples of governmental and corporate influence on what science was done and what results were reported are numerous. For instance, U.S. funding allocated toward pure nuclear physics research decreased dramatically after the fall of the Soviet Union (NRC, 2001). More recently, the Union of Concerned Scientists issued the results of a survey demonstrating "pervasive and dangerous political influence" at the Food and Drug Administration (FDA) (Union of Concerned Scientists, 2006).

The modern practice of science institutionalizes this influence. Drafts of text submitted by scientists for publication in peer-reviewed journals are edited both for style and substantive issues depending on the editorial policies of the journal to which they are submitted. This practice has significantly impacted the direction science takes because it alters methods utilized or topics studied—subtly changing the existing body of research (Toulmin, 1995). Researchers must refer back precisely to this same body of work to justify their next research studies, thereby propagating earlier influences throughout subsequent scientific activities.

The influence of culture on science can be subtle. Kitcher (1995) argued that, in spite of agreement that the ideal scientific discourse should enable the logical structure of arguments to be transparent, idiomatic structures prevent it. Indeed, scientific writers actively use figuration as they attempt to persuade readers. Genes are called "selfish," and the Earth is a "living cell" (Lyne, 1995). Each of these descriptions evokes images and connections unique to each reader. 
Conclusion. The description of the practice of science provided here bears little resemblance to the image of science most students possess when they leave compulsory education. If students' primary experience of science is in school, this mismatch may only be understood to originate in the instructional decisions made by teachers, and in the policy context created by state and federal governments. A description of science instruction as it is commonly practiced, along with the history of education and educational paradigms upon which that instruction rests, will help to explain this apparent mismatch and position this study within a historical and epistemological framework.

\section{Nature of Science Instruction}

\section{Constructivism as the Referent for Science Pedagogy}

The term constructivism is applied to two distinct areas of study. Psychological constructivism is a theory that individuals learn by building meaning from interactions with their environment. Social constructivism is a theory that individuals construct meaning from their interactions with their social environment. As such, social constructivism may be considered to be an adaptation of psychological constructivism. Social constructivism has two important interpretations. Social constructivism describes the way in which disciplines have evolved in the course of human history through communication and negotiation (Phillips, 2000). But social constructivism is also the theory that individuals construct meaning from their social environment. This is because humans are social communicating beings constructing important meaning from interactions with other members of their communities.

As a theory of learning, constructivism has powerful qualities to explain the successes and failures experienced by students and their teachers. Psychological 
constructivism has relevance for education because it transforms the paradigm organizing classrooms from a paradigm which explains teaching and learning as knowledge transmission from teacher to student, into one explaining education as a process by which teachers scaffold experiences from which students control their own knowledge acquisition. Social constructivism enhances this educational paradigm shift, but it also has particular philosophical implications for science education because, in addition to informing both the modes of learning and teaching, social constructivism also informs the way scientific knowledge is created.

Two ideas informing both teaching and learning are central to constructivism: Knowledge is invented and relies on previous knowledge and knowledge is constructed in order to reach goals. Teaching from a constructivist perspective enables the teacher to anticipate students' prior knowledge or misconceptions to inform instruction. Learning in a constructivist environment enables students to gain awareness of the constructed nature of understanding. With this awareness students are enabled to develop a more emancipative, critical relationship with knowledge and its producers (Tobin, 1998)—a relationship with special importance in the context of understanding the nature of science.

\section{Psychological Constructivism}

The philosophical concept that knowledge is constructed either through interaction between mind and world, or during interaction between two minds can be traced back to the Socratic method as a mode of philosophic enquiry as reported by Plato in the Socratic Dialogues (Plato, trans. 1980). Emmanuel Kant was the first philosopher, however, to explicitly discuss the concept that knowledge is "the product of our own cognitive apparatus" (Phillips, 2000, p. 8). Kant's work formed the foundation for 
Piaget's psychological constructivism. Piaget conceived the theory that knowledge does not and cannot have the purpose of producing representations of an independent reality but instead has an adaptive function enabling its user more effectively to pursue his goals (von Glasersfeld, 2005). This perspective is firmly rooted in the pragmatist philosophy of John Dewey which holds that truth is constructed by the interaction between the individual and the natural world and that experimentation could be the central arbiter of truth (Dewey, 1933).

Psychological constructivism describes the relationship between the perception of reality and the reality itself. Bateson (1981, as cited in Larochelle \& Bednarz, 1998) illuminates the central concept:

...all searches for sources of the self "beneath the skin and between the ears" would amount to a project as vain as to ask whether the "me" of the blind person starts as the handle of the stick, halfway up the stick, or at the tip of the stick. (p. 9)

By this, Bateson was arguing that objective reality beyond the self is not knowable because it can only be perceived by the mind through the senses and is thus confounded by them.

This school of thought holds that knowledge cannot be transmitted-neither can it be neutral. Instead, it is constructed, negotiated, motivated by goals, and perpetuated for as long as it organizes its creator's reality. In this perspective, knowledge becomes a tool to be used as individuals work to achieve their goals (Larochelle \& Bednarz, 1998). In addition, previous experiences form the framework from which individuals confront each new experience. 
Psychological constructivism may form the foundation of a variety of teaching paradigms. It may simply be the foundation of a didactic theory a teacher may use to interpret and contribute to the formation of knowledge by informing how and to what extent the teacher communicates knowledge to students. It may also provide an analytic schema with which the teacher anticipates the student's cognitive activity. Communication, in this paradigm, is conceived as a means of serving to "orient" students' efforts at knowledge construction (Larochelle \& Bednarz, 1998). Not only can constructivism inform teachers' understanding of their students' process of cognition, but it may also inform teachers' own conceptions of their understanding of their students' conceptions (Morf, 1998). Constructivist teachers realize that their perceptions of students are as bound to their own prior knowledge and experience as those same students' knowledge of the curriculum are bound to their prior knowledge and experience.

\section{Social Constructivism}

Social constructivism describes how a child, born into a social order, becomes a functioning member of that society (Duveen, 1997). In this perspective, society and social interaction are not merely environmental concepts one learns about, but the mind interacts with social life, creating itself in the process. Emerging from the foundational work by philosophers including Marx and Weber, Vygotsky developed a modern theory of social constructivism. He viewed symbolic thought as a social process-an external dialogue that becomes internalized through social interaction (Vygotsky, 1978).

Vygotsky used the phrase zone of proximal development (ZPD) to describe the cognitive space defined by the actual cognitive level of an individual and the proximal potential for 
development in that domain. In this zone social interactions cause cognitive changes in an individual (Tudge, 1990).

Vygotsky understood learning in a formal school setting as distinctly different from that which occurred spontaneously in everyday interactions. He viewed the school structure as providing the opportunity to form higher cognitive processes such as voluntary attention and logical memory (Hausfather, 1996; Panofsky, John-Steiner \& Blackwell, 1990). A set of higher cognitive functions may be seen to include those associated with scientific reasoning. In order for these functions to be formed, teachers must carefully scaffold instruction that incorporates opportunities for students to practice them in meaningful ways.

\section{The Special Relationship between Constructivism and Science}

The study of science is grounded in constructivism in two ways. First, psychological constructivism explains the relationship between mind and object. It bridges a philosophical gap between rationalists such as Descartes, who viewed knowledge as flowing from clear and indubitable ideas, and empiricists such as Locke, who viewed knowledge as the consequence of sensory experience. Kant (1781/1998) connected these perspectives by asserting that knowledge required both mental effort and sensory experience. Because perception is mediated through the senses, one can never have a direct experience of "reality" (Bredo, 2000). The study of this supposed "reality" is precisely the domain of science.

Second, science is a social act as it is the result of argumentation. Scientific knowledge is accumulated through an antagonistic process of continual contestation until only the simplest and most predictive ideas remain. Tobin (1998) draws the distinction 
between the form, nature and impact of the "taught curriculum" as being at odds with the actual nature of science. If students are to come to understand the true nature of scientific knowledge as being socially constructed, argumentation must have a central role in the science classroom. In order for students to understand how scientific knowledge is accumulated, as well as its tentative nature, they must participate in activity which constructs knowledge in much the same way. Teachers who engage students in activities that are aligned with this view of knowledge construction will likely have greater impact on their students' understandings of NOS.

\section{The History of Science Instruction in the United States}

There are perhaps two permanent trends in U.S. science education-the call for what is now described as constructivist science inquiry experiences for students, and the predominance of its antithesis, the ever-present, didactic textbook centered mode of instruction (DeBoer, 1991). DeBoer traces this dynamic equilibrium over the previous 150 years. Spencer (1820-1903), a prominent nineteenth century British political philosopher posited:

No extent of acquaintance with the meaning of words, can give the power of forming correct inferences respecting causes and effects. The constant habit of drawing conclusions from data, and then of verifying those conclusions by observation and experiment, can alone give the power of judgment correctly. And that it necessitates this habit is one of the immense advantages of science. (Spencer, 1864, as cited in DeBoer, 1991)

Spencer's observation was still valid at the turn of the last century. In 1893 the National Education Association (NEA) recommended three purposes for the study of science: "To 
interest children in nature, to develop mental abilities to investigate carefully, make truthful statements, and to develop a taste for original investigation, and lastly [italics added] the acquisition of knowledge" (DeBoer, 1991, p. 44). In 1909, Dewey argued the same point, recognizing that the instruction of science as a set of facts led to the necessary obfuscation of the subject. Furthermore, the essential nature of science is not as a set of facts but a method of knowing. Dewey argued that only by prioritizing the teaching of science as method over science as subject matter would its potential impact be seen (Dewey, 1910).

From 1917 to 1957 known in education as the "Progressive Era," two distinct but significant developments occurred that impacted science education in the United States. The U.S. public school curriculum changed to provide more practical education for working class people. This period was characterized by socially relevant curriculum and methods enabling students to solve everyday problems. This included the creation of a general science course that would provide an overview of science and train students to observe, imagine, and reason (DeBoer, 1991).

Second, concurrent with the Progressive Era, Thorndike $(1901 ; 1922)$ suggested that students could not transfer knowledge and skills from one situation to another-which implied that direct instruction and repetition was the most effective way to promote cognitive development. Furthermore, a proponent of intelligence testing, Thorndike espoused the theory that real effects of intelligence were measurable. This instructional philosophy fundamentally conflicted with the progressive methods promoted by Dewey. The popularization of Thorndike's pedagogy coincided with the development of standardized achievement testing. The combination of a pedagogy promoting repetition 
of simple tasks and an efficient assessment model within the institutional structure of public schools was a powerful educational force that forced Dewey's progressivism out of mainstream thought (Levin, 1991).

The status quo remained at the end of the Second World War. Teachers still relied on textbooks which were written in a way that discouraged students from thinking critically. Concerns about post-war planning for education prompted renewed discussion regarding the place of critical thinking in the curriculum.

Critical reflective analysis of our courses indicates that many of us are victims of the 'traditional approach' to the teaching of the exact sciences. ...A fair criticism of our present courses is that they are too nearly limited to a 'giving back' by students of information which we, or our textbook writers, deem essential. (Aptekar, 1945, as cited in DeBoer, 1991, p. 106).

Nearly twenty years later, in spite of the Cold War with the launch of Sputnik and the call for preparation of more scientists and engineers, the typical educational experience of students had not changed. A curriculum theorist, Schwab (1962b) critically described the instruction of science "as a nearly unmitigated rhetoric of conclusions [italics in original] in which the current and temporary constructions of scientific knowledge are conveyed as empirical, literal, and irrevocable truths" (p. 24).

This trend has continued into the more recent past. The frequently quoted passage from A Nation At Risk (1983), "If an unfriendly foreign power had attempted to impose on America the mediocre educational performance that exists today, we might well have viewed it as an act of war. As it stands, we have allowed this to happen to ourselves," 
summarizes the perspective of many during the 1980s. A Nation at Risk made specific recommendations for science education that form a refrain in this historical summary: The teaching of science in high school should provide graduates with an introduction to: (a) The concepts, laws, and processes of the physical and biological sciences; (b) the methods of scientific inquiry and reasoning; (c) the application of scientific knowledge to everyday life; and (d) the social and environmental implications of scientific and technological development.

\section{A National Call for Science Inquiry Instruction}

The call for science education to adopt a constructivist pedagogy to most effectively teach students continues to the present day. In 1990, the American Association for the Advancement of Science (AAAS) published Science for All Americans (SFAA), redefining scientific habits of mind, understanding and skill that are “...essential for all citizens in a world shaped by science and technology," (AAAS, 1990, p. xiii). SFAA asserted that the current science curriculum was the accretion of concepts from decades of programs, initiatives and projects. Science instruction had devolved to the state of endlessly covering content without taking the time to provide it with utility or meaning by teaching thinking, questioning and science inquiry skills (AAAS, 1990).

SFAA began with a description of the scientific world view and the nature of scientific inquiry. From this, the perspective of the AAAS could be inferred that scientific inquiry should hold a central role in the understanding of all scientifically literate citizens. SFAA (AAAS, 1990) described five qualities of scientific inquiry: (a) The demand for evidence, (b) the blend of logic and imagination, (c) the explanatory and 
predictive nature, (d) the effort to try to identify and avoid bias, and (e) the nonauthoritarian perspective.

During the 1990's two important sets of standards documents were published, Benchmarks for Science Literacy (Benchmarks) (AAAS, 1993) and the National Science Education Standards (NSES) (NRC, 1996). Science inquiry skills and dispositions permeated both. For example, Benchmarks defines seven Scientific Inquiry standards, eight Scientific Enterprise standards, and three Scientific World View standards (AAAS, 1993). The NSES defined seven Science as Inquiry standards for students, and scientific inquiry was represented in the majority of the teacher standards (NRC, 1996). In addition, the NRC (2000) published a volume highlighting the role of inquiry in science instruction, Inquiry and the National Science Education Standards, that specifically addressed the need for inquiry instruction, as well as the strategies that support inquiry instruction.

Not only do the standards documents call for a reduction in the quantity of science content and introduction of new inquiry content, they also call to some extent for shifting the locus of control in the classroom toward the student and realigning modes of communication. Science for All Americans described science as a complex social activity and described the vital nature of the dissemination of scientific information (AAAS, 1990). The NSES science inquiry standard explicated identifying questions, designing experiments, and communicating and defending scientific arguments among other goals (NRC, 1996). These activities do not typically occur in a standard textbook-centered didactic classroom. 


\section{Description of Science Inquiry Instruction}

\section{Practice Described in National Standards}

The NSES described five essential elements of classroom inquiry. The extent to which teachers enable and encourage students to enact these elements influences the quality of the inquiry learning students experience. In particular, learners should:

a. engage in scientifically oriented questions;

b. give priority to evidence in responding to questions;

c. formulate explanations from evidence;

d. connect explanations to scientific knowledge; and

e. communicate and justify explanations. (NRC, 2000, p. 29)

The NSES (NRC, 1996) also enumerated skills necessary for students to enact scientific inquiry. Student should be able to: (a) Identify questions that guide scientific investigations, (b) design and conduct scientific investigations, (c) use technology to improve investigations and communication, (d) formulate and revise models using evidence and logic, (e) recognize and analyze alternative explanations, and (f) communicate and defend a scientific argument. These six skills operationally define science inquiry for this study. Caution is needed in interpreting this list of skills to mean that all instruction should focus on these qualities, or that students need only these skills. There are a variety of learning environments in which students can learn these skills.

\section{Classroom Science Inquiry Taxonomy}

Colburn (2000) presented a taxonomy of science inquiry pedagogical techniques. He described a spectrum of activities characterized by decreasing levels of teacher control. The taxonomy begins with structured inquiry, in which the teacher provides 
students the materials, questions, and procedures but did not inform them of the outcome beforehand. Guided inquiry reduces the structure of the activity by allowing students to create their own procedure while still being provided the material and questions to be answered. Open inquiry is similar to guided inquiry with the exception that students also formulate their own question to investigate. Open inquiry has the most characteristics of science as it is practiced. Colburn (2000) concluded the taxonomy with the concept of the learning cycle in which students begin with activity to investigate a concept before the teacher introduces it formally. The instruction concludes with students returning to the investigation in possession of the knowledge and perspective provided by the teacher.

Wenning (2005) expanded the work of Colburn and others in his description of a continuum of inquiry-oriented science pedagogy. He placed the various inquiry teaching practices on a continuum of intellectual sophistication and locus of control (Figure 2.1). Discovery learning uses experience to teach content knowledge. The focus of discovery learning is not on the inquiry experience. Teachers use interactive demonstrations to model concepts or skills during which they ask students probing questions eliciting prediction or explanation of phenomenon. Inquiry lessons differ subtly from interactive demonstrations in that the teacher focuses more explicitly on the scientific process involved. Students become actors in inquiry labs. Three levels of inquiry labs are characterized by increasing student ownership of questions and procedures. Guided inquiry labs incorporate teacher-identified problems and leading questions, along with clearly stated learning objectives. Bounded inquiry labs provide a clearly stated learning objective but students are asked to design and conduct an experiment to attain it. Free inquiry labs provide opportunity for students to ask a researchable question and then 
design and conduct appropriate experiments to answer it. Two forms of hypothetical inquiry listed in Figure 2.1 differ from free inquiry in that specific hypotheses are being formulated and tested as contrasted with the less sophisticated question answering process occurring in the free inquiry setting.

\begin{tabular}{|c|c|c|c|c|c|c|}
\hline $\begin{array}{c}\text { Discovery } \\
\text { Learning }\end{array}$ & $\begin{array}{c}\text { Interactive } \\
\text { Demonstration }\end{array}$ & $\begin{array}{l}\text { Inquiry } \\
\text { Lesson }\end{array}$ & $\begin{array}{l}\text { Guided } \\
\text { Inquiry } \\
\text { Lab }\end{array}$ & $\begin{array}{l}\text { Bounded } \\
\text { Inquiry } \\
\text { Lab }\end{array}$ & $\begin{array}{l}\text { Free } \\
\text { Inquiry } \\
\text { Lab }\end{array}$ & $\begin{array}{c}\text { Pure Hypothetical } \\
\text { Inquiry }\end{array}$ \\
\hline Low & \multicolumn{5}{|c|}{$\begin{array}{c}\leftarrow \text { Intellectual } \\
\text { Sophistication } \rightarrow\end{array}$} & High \\
\hline Teacher & \multicolumn{5}{|c|}{$\leftarrow$ Locus of Control $\rightarrow$} & Student \\
\hline
\end{tabular}

Figure 2.1. Spectrum of Science Classroom Inquiry Activities

Even in a constructivist classroom, didactic instruction may be a very effective practice. Depending on the nature of students and content to be learned, inquiry instruction can be very ineffective. America's Lab Report (NRC, 2006) enumerated four principals of instructional design that can help laboratory experiences achieve intended learning goals. Labs should:

a. be designed with clear learning goals in mind;

b. be intentionally placed in the flow of instruction;

c. be designed to integrate the goals of learning content and learning about science inquiry; and

d. incorporate ongoing student reflection and communication. (p. 197)

\section{Science Inquiry Learning Environments}

The activity of science as practiced by the scientific community does have parallels in the classroom; for example, learning communities may be established in science classes in ways similar to those in the scientific community. Crawford, Krajcik, 
and Marx (1999) enumerated seven qualities of classrooms found to make learning communities flourish: (a) Tasks connected to real-world applications generate more collaborative interactions, (b) collaborative interactions increase when participants initiate them, (c) instructional support increases group decision making, (d) group productivity increases when participants have ownership, (e) public sharing of results increases cognitive awareness, (f) interactions with outside experts increases participant investment in a project, and (g) teamwork answering self-generated questions increases participant ownership for outcomes.

\section{Factors Impacting Science Inquiry Instruction}

Promoting the developments of student inquiry skills is perhaps the most complex and challenging instructional task science teachers face. This task requires a dynamic balancing of a multitude of institutional, cognitive, behavioral, temporal, and pedagogical socio-cultural elements. For example, Tretter and Jones (2003) found that increasing the sophistication of an activity and gradually shifting the locus of control toward students may result in significant gains in student inquiry understanding. In a non-equivalent control group quasi-experimental study, Chang and Mao (1999) found that Taiwanese ninth grade earth science students exposed to instruction that required them to interact in small groups, engage in tasks without clear structure and emphasizing active research processes achieved at significantly higher levels and demonstrated more positive attitudes toward the subject matter than students receiving regular instruction in the control group.

Students' cognitive levels and prior knowledge affect the success of inquiry instruction. For example, Kuhn, Black, and Keselman (2000) found that students' incorrect mental models of multivariate causality prevented them from reaping benefit 
from classroom inquiry experiences. Effective instruction may ameliorate this however. Kuhn, Black, and Keselman (2000) implemented an innovative virtual environment in which students solved problems associated with flooding in a town. It simultaneously targeted both mental models and inquiry abilities of middle school students. The researchers found that when students' foundational concepts were targeted, in addition to their surface comprehension, students were able to correctly incorporate inquiry concepts and were able to transfer these to other contexts.

There is evidence that classroom environment has a significant impact on students' science inquiry learning. Nolen (2003) constructed a hierarchical linear model (HLM) explaining students' perceptions of their science classrooms. Students' shared perception that the class was fast paced and focused on right answers was negatively related to science achievement while the perception that class was focused on learning and honored independent thinking was positively related to achievement. In an ethnographic study of a high school physics classroom, Kelly and Chen (1999) found that level of scientific discourse displayed in students' writing was related to the way teachers framed activities and social practices present in the classroom environment over time.

Research has shown that students' role orientation interacts with their perception of the classroom environment. For example, Nolen (2003) discussed how task-oriented students responded differently from ego-oriented students to a science classroom environment. She applied Nichols' (1989) definition of task-orientation as holding mastery as an important personal goal and ego-orientation as "striving to demonstrate superior ability" (p. 348). Ego-oriented students responded to activities that promoted the most direct route to academic success as compared with other tasks and resisted those 
tasks perceived to carry higher risk. Qualities of these high-risk activities included openended or fuzzy questions, group work, and self-directed learning. Task-oriented students on the other hand, responded to the open-ended group work that typifies the actual practice of science.

Some aspects of the science community may actually hinder the development of a learning community in a classroom. Children from groups typically underrepresented in science, technology, engineering, and mathematics (STEM) fields often do not respond well to the competition and social comparison that characterizes the actual science community (Nolen, 2003). Even when accounting for socio-economic status, these students from these demographic or cultural groups have been described as needing a more supportive environment in order to flourish (Ferguson, 2002; Ogbu, 2003). Von Secker (2002) found that although inquiry-based teacher behaviors were associated with increases in overall student science achievement, they were also associated with increases in an achievement gap between students of differing demographic profiles.

Other characteristics that typify the socio-economic environments in which many minority children learn also impact their capacity to learn science. In an analysis of research on urban science education issues, Calabrese-Barton (2002) identified three dimensions categorizing issues that impact the science achievement of urban minority children: Equity, social justice, and sense of place. Equity in this analysis is expanded to include not only equity of school monetary and material resources but also resources of teacher training and experience. Students in urban schools frequently have uncertified or poorly qualified science teachers (Darling-Hammond, 1999). This issue of equity is compounded, however, as urban districts typically have large entrenched bureaucracies 
and layers of distrust between administration and teachers may have accumulated over the years (Blumenfeld, Fishman, \& Krajcik, 2000; Kahle, Meece, \& Scantlebury, 2000). Issues of social justice may impact the achievement of urban children through low expectations, student resistance, and teacher knowledge (Griffard \& Wandersee, 2001; Tobin, Roth, \& Zimmerman, 2001). Researchers describe how cultural expectations at home create barriers to success in science by honoring a child's labor more than his education (Calabrese Barton \& Yang, 2000). Teachers may ameliorate these problems by making purposeful connections between the intended science curriculum, and science as it understood personally and within the culture of the family (Hammond, 2001).

\section{Current State of Inquiry Instruction}

The Committee on High School Science Laboratories of the NRC was commissioned to study the current state of inquiry experiences in U.S. high schools and make recommendation for future steps (NRC, 2006). This report concluded that inquiry experiences in U.S. schools consist of laboratory instruction not focused on clear learning goals, and use materials that emphasize procedural details but leave students uncertain about what they are supposed to learn.

This is likely caused by the confluence of two factors. Teachers are often poorly prepared to provide authentic inquiry experiences - having little experience in either their university science or science education preparation (Brown \& Melear, 2006). In addition, textbook science is presented to students as absolute and impartial (Schwab, 1962b). Teachers embody the nature of the knowledge. When teachers practice presents science as a litany of facts, they may produce the inappropriate presentation of science as merely factual causing students to incorrectly understand the role of scientists merely as creating 
authoritative objective knowledge. This is opposed to social or spiritual knowledge which is easily understood to be defined subjectively (Desautels, 1998). This incorrectly positions science outside the experience of most students, limiting its power to impact their lives.

\section{Impacting Inquiry Abilities in the Classroom}

\section{Assessing Students' Inquiry Knowledge and Ability}

The NSES (NRC, 2000) calls for assessment of three aspects of learning in inquiry-based classrooms: What students know and understand, what information is "fuzzy or missing," and what students can do with what they know. Assessment should determine whether students are able to generate questions, develop explanations, design and conduct investigations, and use data to support or reject explanations.

Assessment of science inquiry is particularly challenging for teachers because science inquiry is a complex process incorporating conceptual knowledge, analytic skills, and behaviors. Furthermore, assessment is complicated by its diverse purposes:

Diagnostic, formative, summative, comparative, in support of professional development, or for program development (Hein \& Lee, 2000). In support of science inquiry learning, Hein and Lee (2000) recommend assessments in which students are asked to provide some of the information rather than simply responding to a prompt, or to demonstrating skills or use materials.

\section{Impact of Science Inquiry Instruction on Inquiry-Related Skills}

A significant body of work exists in science education research supporting the impact of inquiry instruction on inquiry related skills. In a summary of research findings, Haury (1993) listed positive impacts on students': (a) Laboratory skills, (b) graphing, and 
interpreting data; (c) scientific literacy and understanding of science processes; (d) procedural knowledge; and (e) construction of logico-mathematical arguments. Tretter and Jones (2003) reported that although a science-inquiry instructional approach did not significantly impact student standardized test scores, it did positively impact high school student engagement and classroom grades and created more uniform classroom performance.

Suits (2004) found that students who had received inquiry-based laboratory instruction as opposed to standard confirmatory laboratory instruction scored higher on tests of six investigating skills. Hofstein, Navon, Kipnis, and Mamlok-Naaman (2005) confirmed these results in Israel. This research team studied high school chemistry students who were taught either didactically or through an inquiry-based approach. The inquiry instruction classes (experimental group) asked more extensive and higher quality questions after reading a science journal article than did the control group. Middle school students exposed to a science inquiry summer workshop showed significantly greater interest in science and in pursuing science careers than those who applied but were not admitted to the program (Gibson \& Chase, 2002). Johnson and Lawson (1998) reported that student reasoning ability explains a greater proportion of the variance in performance of students in expository college biology classes than student science reasoning ability did in inquiry based classes.

\section{Impact of Inquiry Instruction on Content Knowledge}

Inquiry-based instruction has been shown to positively impact student science content knowledge. For instance, Chang and Mao (1999) found a positive impact of inquiry-group instruction on Taiwanese students' content knowledge and attitudes toward 
earth science. In her analysis of the National Educational Longitudinal Study (NELS:88) data, von Secker (2002) created a hierarchical linear model (HLM) of the impact of teacher inquiry behaviors on student science achievement. She found that on average, students' science achievement increased by 0.58 standard deviations for each standard deviation increase in the emphasis placed on inquiry by their teachers. An important detail should be emphasized however. The format of the assessment instrument may cause it to be insensitive to this effect, however, because many standardized instruments do not assess science inquiry skills (NRC, 2006; Tretter \& Jones, 2003).

\section{Relationships with Teacher Characteristics}

Experienced science teachers have higher dispositions toward the pedagogical use of inquiry than their novice counterparts (Damnjanovic, 1999; Flick, 1995; Roehrig \& Luft, 2004). Significant impact of teacher professional development in science inquiry has been shown to exist if the professional development is formatted well (Jeanpierre, Oberhause \& Freeman, 2005; Marlow \& Stevens, 1999), and of sufficient length (Supovitz \& Turner, 2000). However, ensuring teacher understanding of NOS will not guarantee it is enacted in their classrooms. Abd-El-Khalick, Bell, and Lederman (1998) found that several factors mediated between teachers' understanding of NOS and their classroom practice. Among these are teacher intentions, teacher content knowledge, teacher pedagogical knowledge, students' needs, teacher autonomy, and time. Also, the correct perspective of NOS will not necessarily be created by students' experience of "doing science." 


\section{Teacher Efficacy and Personal Agency}

Teaching is an act that occurs within the physical space and time of the classroom where activity is planned and executed, resources are gathered, organized and used, communication is exchanged, and behavior modulated. But teaching also occurs in the intellectual space shared in thoughts, perceptions and emotions of teacher and student, as knowledge and skill are constructed and linked. Because so much of this endeavor occurs in realms that may not be seen or touched but are vitally linked to real objects and events, teacher beliefs about their capacity to create changes in students' understandings play an important role determining the teacher's success. For this reason, discussion of the role of teacher efficacy and personal agency in teacher activity and the consequent level of student success in science classrooms is important. Self Efficacy

The foundation of the self efficacy construct originated in the concept that the location of locus of control could be perceived to be either internal or external (Rotter, 1966). Armor and others (1976) found locus of control to be a powerful factor relating teacher variables with student learning. In 1977, Bandura extended this concept by developing the construct of self efficacy as a person's estimate of his capacity to successfully reach a particular goal. Bandura's original work differentiated self efficacy from outcome expectation, which is a person's expectation that a particular activity will lead to a specific outcome. Self efficacy beliefs relate to a person's sense that he can perform a set of actions while outcome expectation describes a person's sense that a set of action will lead to a particular result. Both are important concepts explaining a person's decision to undertake a specific course of action (Bandura, 1977). 
Teachers' sense of self efficacy has been shown to be an important predictor of many educational outcomes. For instance, self efficacy has been related to student achievement (Armor et al., 1976), levels of teacher planning and organization (Allinder, 1994), and willingness to try new methods to meet the needs of students (Berman, McLaughlin, Pauly, \& Zellman, 1977). Furthermore, higher self efficacy was positively related to teachers' enthusiasm for teaching (Allinder, 1994), and commitment to teaching (Coladarci, 1992).

\section{Personal agency}

Personal agency concerns how people exercise control over their lives (Goddard, Hoy, \& Woolfolk-Hoy, 2004). Personal agency extends self efficacy to include both capability beliefs, which are synonymous with self efficacy, and context beliefs which are evaluations of the responsiveness of one's environment (Ford, 1992). Bandura (2006) described four core properties of personal agency: (a) Intentionality, the capacity to form intentions along with plans and strategies for their realization; (b) forethought, the capacity to bring anticipated outcomes to bear on current actions; (c) self-reactiveness, the capacity to self-start linking thought and action; and lastly (d) self-reflectiveness, the metacognitive ability to change courses of behavior based upon assessment of the effectiveness of that behavior to realize a goal. Bandura (2006) extended the definition of personal agency to include three forms: Individual, proxy, and collective. Proxy and collective agency describe those situations in which a person does not have the personal control over the conditions in which he functions but can leverage control by influencing others (proxy agency) or working with others (collective agency). 
Self efficacy and personal agency beliefs have been shown to have important relationships to teaching practice. Riggs and Enochs (1990) applied Bandura's (1977) self efficacy construct to the development of the Science Teacher Efficacy Beliefs Instrument (STEBI). Ramey-Gassert, Shroyer, and Staver (1996) used the STEBI to study elementary science teachers' teaching efficacy beliefs. They categorized three types of factors impacting science teaching efficacy: Antecedent, internal, and external. Antecedent factors include teacher preparation and experience. Internal factors include teacher interest and attitudes toward science. External factors include student, workplace, and community variables. Cannon and Shaarmann (1996) found that cooperative field experiences increased the science teaching efficacy of preservice elementary teachers. Enochs, Schaarmann, and Riggs (1995) found that elementary teachers' self efficacy was correlated with science courses taken, perceived teaching effectiveness, and instructional practice. Haney, Lumpe, Czerniak, and Egan (2002) found that teachers with high personal agency beliefs were more likely to design instruction incorporating inquiry, attend to student prior knowledge, and use available resources. These teachers are also more likely to present science content that was appropriate for their students in an interesting and engaging way.

\section{The National Board for Professional Teaching Standards}

"A Nation at Risk: The Imperative for Educational Reform" (National Commission on Excellence in Education, 1983) was produced by a commission of university presidents and professors, corporate executives, school board members, education commissioners, former governors, school administrators and teachers. This 1980's report responded to a perceived state of disrepair in American schools. The 
commission had several recommendations with respect to the teaching profession. Among them were:

1. Salaries for the teaching profession should be increased and should be professionally competitive, market-sensitive, and performance-based. Salary, promotion, tenure, and retention decisions should be tied to an effective evaluation system that includes peer review so that superior teachers might be rewarded, average ones encouraged, and poor ones either improved or terminated.

2. School boards should adopt an 11-month contract for teachers. This would ensure time for curriculum and professional development, programs for students with special needs, and a more adequate level of teacher compensation.

3. School boards, administrators, and teachers should cooperate to develop career ladders for teachers that distinguish among beginning instructor, experienced teacher, and master teacher. (NCEE, 1983)

In 1986 in response to this call, A Nation Prepared: Teachers for the $21^{\text {st }}$ Century recommended the creation of a national body whose purpose was to create rigorous standards for accomplished practice in the teaching profession and the creation of a process by which expert teachers could receive this certification (Carnegie Forum on Education and the Economy, 1986). The consequence of this call was the endowment in 1987 of the National Board for Professional Teaching Standards (NBPTS) by the Carnegie Foundation. The NBPTS board of directors was composed of a majority of classroom teachers, but also included school administrators, higher education officials, 
school board leaders, governors and state legislators, business and community leaders, and teacher union leaders. The board's mission was to:

1. Advance the quality of teaching and learning by maintaining high and rigorous standards for what accomplished teachers should know and be able to do;

2. provide a national voluntary system certifying teachers who meet these standards; and

3. advocate related education reforms to integrate National Board Certification in American education and capitalize on the expertise of National Board Certified Teachers (NBPTS, 2006a).

The NBPTS began its work by publishing What Teachers Should Know and Be Able to Do (NBPTS, 2002). This document enumerated five fundamental descriptions or Core Propositions that the NBPTS asserted were the foundation of accomplished teaching: (a) Teachers are committed to students and their learning; (b) teachers know the subjects they teach and how to teach those subjects to their students; (c) teachers are responsible for managing and monitoring student learning; (d) teachers think systematically about their practice and learn from experience; and (e) teachers are members of learning communities.

\section{NBPTS Standards}

The NBPTS constituted committees comprised primarily of classroom teachers but including experts in child development, teacher educators and discipline-specific experts to write standards for each certificate offered (NBPTS, 2003a, 2003b). Each standards document consists of between ten and fifteen descriptions of accomplished 
teaching practice grounded in the five Core Propositions. A draft of each standards document underwent repeated revisions before being approved by the NBPTS Board of Directors. Each document was then widely disseminated within the education community for public comment and subsequent revision before its final form was adopted by the NBPTS Board of Directors (NBPTS, 2006b).

\section{NBPTS Science Portfolio}

Between 1987 and 1992, The NBPTS built a national certification system upon this set of five core propositions that enabled teachers to receive formal recognition for accomplished practice in the same way that other professions do. This provided a route for teachers to advance their careers without following typical paths into counseling or administration (NBPTS, 2006c). The first NBPTS certificates were available in 1994. The Adolescent/Young Adult Science (AYA Science) certificate, the high school level certification, was first available in 1998 and first Early Adolescence Science (EA

Science) certificates for middle school teachers were issued in 1999. Both are still offered to teachers.

To earn an NBPTS Adolescent/Young Adult Science certification, teachers must prepare a portfolio containing four entries: (a) Teaching a Major Idea Over Time, (b) Active Science Inquiry, (c) Whole Class Discussions About Science, and (d) Documented Accomplishments. The first three entries for candidates are developed from classroom instruction. Candidates must provide evidence of accomplished practice including instructional documents, student work samples and videotapes of science instruction. The fourth entry includes evidence of the teacher as learner, communicator and collaborator in which the candidate must show evidence of out-of-class interactions 
that extend and complement the teacher's presence in the classroom (NBPTS, 2004a). The NBPTS Early Adolescence Science certificate has similar requirements (NBPTS, 2004b).

Each entry has limitations on number of pages for description, analysis, and reflection, and artifact evidence. The final component completed by a candidate for certification is a standardized on-demand assessment containing six pedagogical content knowledge questions, each of which must be answered by a candidate within 30 minutes. This assessment is taken at an Educational Testing Service (ETS) sanctioned standardized testing center. All candidate responses to prompts must be word processed. Portfolio entries are scored on a four-point scale by trained assessors. Each classroom entry is worth $16 \%$ of the total score, the documented accomplishments entry is worth $12 \%$ of the total score, and the assessment center is worth $40 \%$ of the total. In order to certify, the candidate must receive an aggregate score of 2.75 (NBPTS, 2004a, 2004b). Research on National Board Certification Correlation with Teacher Quality

A number of studies to date have examined the filtering nature of National Certification, comparing the achievement of students of those teachers who became certified with those of teachers who attempted but did not. A study of elementary students in Arizona showed that students of NBCTs received the benefit of the equivalent of one extra month of instruction over the course of a year compared to students of nonNBCTs. The researchers' ex post facto causal comparative longitudinal design used teacher and principal surveys to gather data on teacher and school characteristics and longitudinal student achievement data gathered from the SAT-9 in grades three through six (Vandevoort, Amrien-Beardsley, \& Berliner, 2004). 
A study of North Carolina Middle Childhood Generalist and Early Adolescent Language Arts NBPTS certified teachers identified thirteen dimensions across which to compare teachers including (a) use of knowledge, (b) pedagogical content knowledge, (c) problem solving strategies, (d) adaptation of goals for diverse learners, (e) decision making, (f) creating challenging objectives, (g) creating classroom climate, (h) perception of classroom events, (i) sensitivity to context, (j) monitoring and providing feedback, (k) testing of hypotheses, (l) respect for students, and (m) passion for teaching. Researchers examined the practice of 65 teachers, 32 of whom held NBPTS certification. Data collected included teacher lesson plans, classroom observations, and interview transcriptions. Student data included student work sarnples and student responses to prompts provided by the researchers. Researchers analyzed the data blind to the certification status of the teachers. Analysis of teacher data indicated NBCTs had more expertise than their non-NBCT counterparts on all thirteen teacher dimensions with eleven of the thirteen comparisons significantly different (Bond, Smith, Baker, \& Hattie, 2000).

As of 2007, studies have shown that teachers who merely attempted certification but did not certify were more effective than those who did not. A study of 108,000 student records from the Miami-Dade school district revealed a rank ordering of student achievement as measured by end-of-year exams with highest achievement belonging to students of NBCTs, smaller gains for NBCT candidates, and less for teachers who failed or withdrew from the NBPTS process. Of importance to this study is the observation that students were not randomly paired with teachers. Descriptive statistics in the study indicated that more able students were more likely to be paired with NBCTs than other 
teachers. In addition, NBCTs were more likely to be experienced and have higher degrees than other teachers in the sample. A significant benefit of having an NBPTS certified teacher remained even after accounting for the effect of these other teacher characteristics (Cavalluzzo, 2004).

Goldhaber and Anthony (2005) found that National Board Certification was an effective indicator of teacher quality but found no evidence of the certification process itself impacting teacher quality. Their study utilized an ex post facto causal comparative longitudinal design, studying the impact of teacher certification on elementary student end-of-course assessments in North Carolina over three years. Results included the finding that NBCTs appear to have a greater impact on younger minority children and that the impact seems to be robust against the possibility that students are non-randomly assigned to NBCTs. Some confounding results of this study include evidence that teachers are less effective during the year of their certification and that they may be less effective after certification. The authors indicate that the latter result could be an artifact of a small post-certification sample or that teachers are spending more time with out-ofclassroom tasks after certification. These studies' limitations include the use of regression to examine nested data which violates the independence assumption. This tends to bias results making the effect size seem larger than it is (Raudenbush \& Bryk, 2002).

The literature supports the inference that NBCTs foster greater depth of understanding in their students (Smith, Gordon, Colby, \& Wang, 2005). Smith and colleagues found that NBCTs out-performed their non-NBCT colleagues on six of seven student outcome measures. Methodology in this study examined both the depth of work from a randomly chosen sample of each teacher's students and the depth of the teacher's 
aims for student achievement as were expressed in planning documents. Smith et al. found that the majority of student outcomes were at the surface level, regardless of teacher certification status. The researchers also found, however, that students of NBCTs were more than twice as likely to achieve "deep" learning outcomes. In addition, the researchers found that the students of NBCTs scored higher on six measures of writing skill than those of the non-certified counterparts.

However, some studies have not found differences between teachers based on NBCT status. Stone (2002) applied the Tennessee Value-Added Assessment System (TVAAS) to 16 NBCTs teaching in Tennessee. The teacher-effect scores are based on the Terra-Nova standardized assessments administered during elementary school years. Not all tests are given to all students in any given year so the number of teachers who could be included was limited. Stone's analysis used the Chattanooga standard of students achieving at $115 \%$ of the national norm gain as evidence of exemplary teaching. He found NBCTs were no more likely to have students score at this level than other teachers. Sanders, Ashton, and Wright (2005) compared the end of grade scores for three levels of North Carolina teachers associated with National Board Certification (NBC) against the general population of teachers in North Carolina. The three levels of teachers were: NBCTs; teachers who intended to pursue NBC in the future; and teachers who had attempted and failed. The researchers' hierarchical model found greater variation within each group of teachers than between groups, and limited significant benefit to students on end of year tests, using a gain-score method including previous year's scores as covariates. Reviewers cited limitations of this study which included a change in the endof-year assessment that occurred during the study (results were based on change-scores), 
insufficient reporting of sampling and other techniques, and inclusion of conclusions as results that could indicate poor sampling (NBPTS, 2006d).

\section{National Board Certification as Effective Professional Development}

The National Board Certification process includes characteristics that, if examined separately, are recognized in the research literature as effectively enabling change in professional practice. Preparing for and writing the National Board Certification (NBC) portfolio is inherently analytical and reflective. The common method applied by NBC candidates completing the certification process is to work with other candidates in a collegial group under the tutelage of a mentor who may or may not be an NBCT (NBPTS, 2001). Aspects of this model have been described as positively impacting teaching performance. Participating in collegial groups has been shown to be an effective method to change teachers' practice (Keedy \& Robbins, 1993). Reflective practice results in increased student performance and higher levels of teacher satisfaction (Giovannelli, 2003; Schön, 1987; Dewey, 1933).

There are relatively few studies that have probed how National Board certification affects teachers who attempt it. Lustick (2002) found that the National Board Certification process helped teachers manage uncertainty of outcomes, complexity of task and professional isolation. Lustick (2002) posited a framework that categorized the impact the certification process might have on teachers of differing initial skill sets. Novice teachers may perceive significant change while expert teachers may perceive less change as a result of attempting certification. Irwin-Beck (2002) found that teachers who had pursued National Board Certification reported it to be a valuable professional development activity, but there was no difference in the opinions of veteran and non- 
veteran teachers regarding their perspective of its effectiveness. Serafini (2001) described the National Board certification process as one of "...aligning to the vision of accomplished practice..." Some research supports the process of National Board certification as shifting teachers' focus from activity-based to standards-based instruction (Taylor, 2000).

Lustick and Sykes (2006) investigated the impact of the National Board Certification process on high school science teachers. The researchers asked candidates to respond to questions and analyze student work and videotape evidence at three points during their certification attempt: Before they began serious work, while they were working, and the year after they finished but before they knew if they had certified. Lustick and Sykes (2006) found the most significant improvement in candidates' understanding of assessment and inquiry. One limitation of this study is that the researchers did not actually observe the classroom practice of candidates. An assertion in this study that National Board Certification impacts teacher practice would be stronger if it was associated with observations of changes in pedagogical skill in a classroom context.

\section{Literature Review Conclusion}

This review of literature has shown science inquiry is a specialized mode of inquiry, uniquely connected to the constructivist paradigm of knowledge creation. It has also been shown that students benefit generally from science inquiry instruction, and that in spite of calls for it over the previous 150 years, science inquiry instruction has not been commonly practiced in U.S. schools. The success of the NBPTS certification process has been shown to identify accomplished teachers and to be an effective professional 
development activity. In particular, the NBPTS certification process has been shown to positively impact science teacher assessment and science inquiry capability of students in P-12 classrooms.

To date, no studies exist, however, connecting NBPTS certification with teacher understanding of the nature of science, teacher inquiry teaching practice, or student science reasoning and NOS understanding. This study investigated these areas. In particular, this study's purpose is to answer the following questions:

1. How does National Board Certified science teachers' knowledge of the nature of science differ from that of their non-certified counterparts?

2. How do the frequencies of student science inquiry behaviors supported by the learning environments created by NBCTs differ from those created by their non-NBCT counterparts?

3. What is the relationship between the frequency of students' science reasoning behaviors and their science reasoning and understanding of the nature of science?

4. What is the impact of teacher perceptions factors impacting curriculum and limiting inquiry on the existence of inquiry learning environments? 


\section{METHODOLOGY}

\section{Rationale}

Measuring the impact of teacher professional qualities on student skills and understanding is a difficult endeavor at best. As students progress through school, their time with individual teachers represents an ever decreasing proportion of their life and experience. The confounding effect of prior life and formal education experience increases with time as well so discerning the impact of an individual teacher or intervention becomes increasingly difficult. For these and other reasons, education studies should be designed with carefully considered goals in mind and their results should be stated clearly, bounded by the limitations inherent within the chosen design.

The nature of a study's research questions should drive the choice of methodology most appropriate to answer them (Ercikan \& Roth, 2006). Qualitative studies of educational experiences quickly become entangled in the many relationships and interactions that connect each child to his world-a web of connections that grows increasingly complex as years of life and educational experience increase. Quantitative education studies of these experiences are bound by the diminishing effect size, the proliferation of confounds and counterfactuals, and the nested nature of human studies data.

Qualitative studies position a researcher as a lens through which the study is conducted. The goal of the qualitative researcher is to describe or evaluate the research 
setting as it is, accepting that his presence impacts the "truth" being investigated, and that both he and his participants may be changed as a result of the interaction (Rossman \& Rallis, 2003). Qualitative research is typically descriptive or evaluative (Rossman \& Rallis, 2003). Qualitative studies do not lend themselves to searching for generalizable trends and effects because they occur in naturalistic settings and are situated in the particular context in which the phenomenon is being studied (Rossman \& Rallis, 2003). In addition, such studies are very labor intensive which makes them difficult to enact on a large scale.

Quantitative studies on the other hand, originate in the positivist stance that external reality exists independently of the observers, is observable, and knowable. Quantitative researchers often claim to be able to separate themselves from the measurement, believing that controlled experimental conditions can uncover underlying patterns that are applicable to other settings. In order to obtain this generalizability of results, quantitative studies sacrifice many of the relationships and connections that are essential to make their results resonate with the experienced lives of the reader.

Social science research may be thought of as describing an underlying reality in much the same that an artist does as he translates his perspective into a work of art. The artist balances perception and interpretation as he strives to express a fundamental experience of reality. In order for the social science researcher to paint an adequate picture of a student's experience as he learns science-a picture that is useful in the search for ways to make students' experiences in science class more meaningful-the researcher cannot ignore either of the conflicting realities that define the positivist and phenomenological perspectives. A pragmatic stance must be assumed that enables use of 
the strength each method provides to answer questions while simultaneously looking to the other to ameliorate its weaknesses (Tashakkori \& Teddlie, 1998). The quantitative approach draws the outline of what is occurring, while the qualitative approach provides the hue and value.

This study's methodology arose from the researcher's recognition that all of the essential validity of educational studies cannot be generated through controlled experiments or qualitative studies alone (Tashakkori \& Teddlie, 1998). The more complex the interactions studied, the more difficult it is to control the setting, or even discern the underlying principals governing it. Clearly there are some common behavior characteristics and patterns that are shared among all humans which can and should be measured. Just as clearly every human interaction is uniquely constructed and situated and cannot be adequately described by numerical trends (Rossman \& Rallis, 2003). One of the fundamental qualities of the human condition is its idiosyncratic situated nature. For this reason, studies that begin to answer the range of questions one might ask about any aspect of the human condition need to include both quantitative and qualitative approaches and analyses. Mixed-method designs enable the researcher to utilize the strengths of quantitative and qualitative approaches simultaneously (Tashakkori \& Teddlie, 1998).

\section{Overview of Methodology}

Description of this study's sample, methodology, instrumentation, and analysis is organized around the four research questions (see Appendix A). A preliminary section operationalizes the latent constructs being investigated. Before specific questions are addressed a general discussion is presented describing the study design, population, 
sampling strategy and subsequent teacher and student samples. Sections describing the specific methodology used to answer each research question include discussion of the instrumentation, analysis, and issues associated with that question. Discussion concludes with sections describing validity concerns and limitations of the study.

\section{Science Reasoning and NOS Understanding Operational Definitions}

For this study, science inquiry learning is defined by the six abilities necessary to do scientific inquiry according to the National Science Education Standards science inquiry standards: (a) Identify questions and concepts that guide scientific investigations, (b) design and conduct scientific investigations, (c) use technology and mathematics to improve investigations and communications, (d) formulate and revise scientific explanations and models using logic and evidence, (e) recognize and analyze alternative explanations and models, and (f) communicate and defend a scientific argument (NRC, 1996). Science inquiry learning was also defined by an understanding of the nature of science underlying the application of these abilities (NRC, 1996). For this study, this definition of science inquiry learning does not include specific measurement skills or knowledge of scientific facts.

Because one assumption of this study was that a learning environment supportive of the acquisition of science inquiry skills and understanding is one that frequently engages students in behaviors described by the NSES science inquiry standards, students' science inquiry skills were operationalized in this study as the ability to control variables, differentiate between observations and inferences, and design experiments.

Understanding the nature of science was operationalized by the extent to which respondents expressed agreement with "more informed views" regarding six constructs: 
(a) Observation and inferences, (b) change in scientific theories, (c) scientific laws vs. theories, (d) social and cultural influence on science, (e) imagination and creativity in scientific investigations, and (f) methodology of scientific investigations.

Study Design

This was a multi-level mixed-method study. The teacher was the unit of analysis, but important information about teacher effectiveness was gathered by examining student level outcomes. To account for this complexity, analyses were completed of both teacher and student variables. At the teacher level, this was a simple correlation design comparing teacher behaviors between teacher groups defined by NBCT status, experience level, and non-education degree level.

At the student level this was a quasi-experimental study using an untreated comparison group design with dependent pretest and posttest samples (Shadish, Cook \& Campbell, 2002). The treatment in this design was having an NBCT as a science teacher. The control group consisted of students taught by non-NBCTs (both experienced and inexperienced). The student groups were not randomly assigned (See Figure 3.1.).

$\begin{array}{cccc}\mathrm{NR} & \mathrm{O}_{1} & \mathrm{X} & \mathrm{O}_{2} \\ \mathrm{NR} & \mathrm{O}_{1} & \mathrm{O}_{2}\end{array}$

Figure 3.1. Quasi-experimental Study Design

The use of a student pretest served to account for any initial differences between the student groups due to lack of random assignment. In this design, selection bias was presumed to be present, but the pretest enabled exploration of its size and direction. Several other threats to validity existed. The selection-instrumentation threat occurred if the assessment instruments were differentially sensitive to members of different groups. Selection-regression threats existed if subjects were selected for group membership by 
virtue of some special merit. In these cases, they may have tended to score less extremely on other measures. This was not an issue in the present study because while NBCT status determined group membership, NBCT status was associated with accomplished teacher practice on the NBPTS assessments and was not necessarily associated with instruments used at this level measuring student performance. Finally, selection-history continued to be a threat because no control existed for the experiences of subjects between pretest and posttest (Shadish, Cook, \& Campbell, 2002).

This study may be described as mixed-model design because both quantitative and qualitative data and analyses were employed. Within the taxonomy established by Tashakkori and Teddlie (1998) it was as a Type VII, or parallel mixed-model study. This was because data collection and analyses were simultaneously qualitative and quantitative. Qualitative data provided direction for analytic decisions (such as which teachers to interview), triangulated with the conclusions of the quantitative analysis, and provided explanation for results.

\section{Data Collection Schedule}

Once enrolled, full participation in this study consisted of completing two phases of documentation (Table 3.1). The first phase occurred in early October 2006. It consisted of assessments of both teacher and student understanding of the nature of science (NOS) using the Student Understanding of Science and Science Inquiry (SUSSI) instrument (Liang, et al, 2006), as well as student understanding of science reasoning using Lawson's Test of Scientific Reasoning (LTSR) (Lawson, 2000). Both instruments are described later in this chapter. The second phase occurred between early December and late January, depending on the participating teacher's schedule. Measurements during this 
phase consisted of surveys of student and teacher perspectives of classroom science inquiry behaviors in addition to teacher perspectives of classroom and school environments, and a reassessment of student science reasoning and NOS understanding. In this description the concept of assessment phase is used rather than that of pre/post assessment because the first set of assessments occurred well into cycle of instruction and did not occur before or after any predetermined intervention. Figure 3.2 displays how data sources flowed into various analyses completed for this study. Particular instruments shown in the figure are described in detail in the following sections describing research question-specific methodology and instrumentation. Rectangles represent data sources and circles represent steps of analysis. Shaded areas represent quantitative data or analyses while unshaded areas represent qualitative data or analyses.

Table 3.1

Phases of Data Collection

\begin{tabular}{lll}
\hline \multicolumn{1}{c}{ Data } & \multicolumn{1}{c}{ First Phase } & \multicolumn{1}{c}{ Second Phase } \\
\hline Student NOS Understanding & Student SUSSI & Student SUSSI \\
Student Science Reasoning & Student LTSR & Student LTSR \\
Teacher NOS Understanding & Teacher SUSSI & \\
Student Perspectives of & & Student ESIS \\
Classroom Science Inquiry & & \\
Behaviors & & Teacher ESIS \\
Teacher Perspectives of & \\
Classroom Science Inquiry & \\
Behaviors & & \\
Teacher Education and & Study enrollment form and \\
Experience & Teacher ESIS \\
Teacher Perspectives of & & Teacher ESIS and \\
Classroom Learning & Classroom Information \\
Environment & Sheet \\
\hline
\end{tabular}




\section{Study Population}

$N B C T s$. This study compared the science inquiry teaching practices of the population of Adolescent/Young Adult Science (AYA Science) and Early Adolescence Science (EA Science) NBCTs in Kentucky against the population of Kentucky teachers as a whole. The participation of all Kentucky AYA Science and EA Science NBCTs was sought because there were fewer than 40 practicing AYA Science and 29 practicing EA Science NBCTs in the state (http://www.nbpts.org/resources/nbct_directory, 2006).

Non-NBCTs. Two comparison groups were formed from the other high school and middle school science teachers in Kentucky. One group was formed from teachers matching the NBCTs on years of experience and school demographics with priority given to matching years' experience. The other group was formed from novice teachers with fewer than five years of teaching experience and matched on school demographics. This definition of novice teacher was derived from the NBPTS restriction that candidates must have more than three years teaching experience in order to be eligible to pursue NBPTS certification (NBPTS, 2004a, 2004b).

\section{Sampling Strategy}

Because the goal of this study was to find and report results that might be generalized to other teachers who did not participate in the study, teachers selected were as representative of the general population of teachers as possible (Tashakkori \& Teddlie, 1998). A purposive sample of Kentucky middle and high school teachers stratified by National Board Certification status and experience level was selected. 


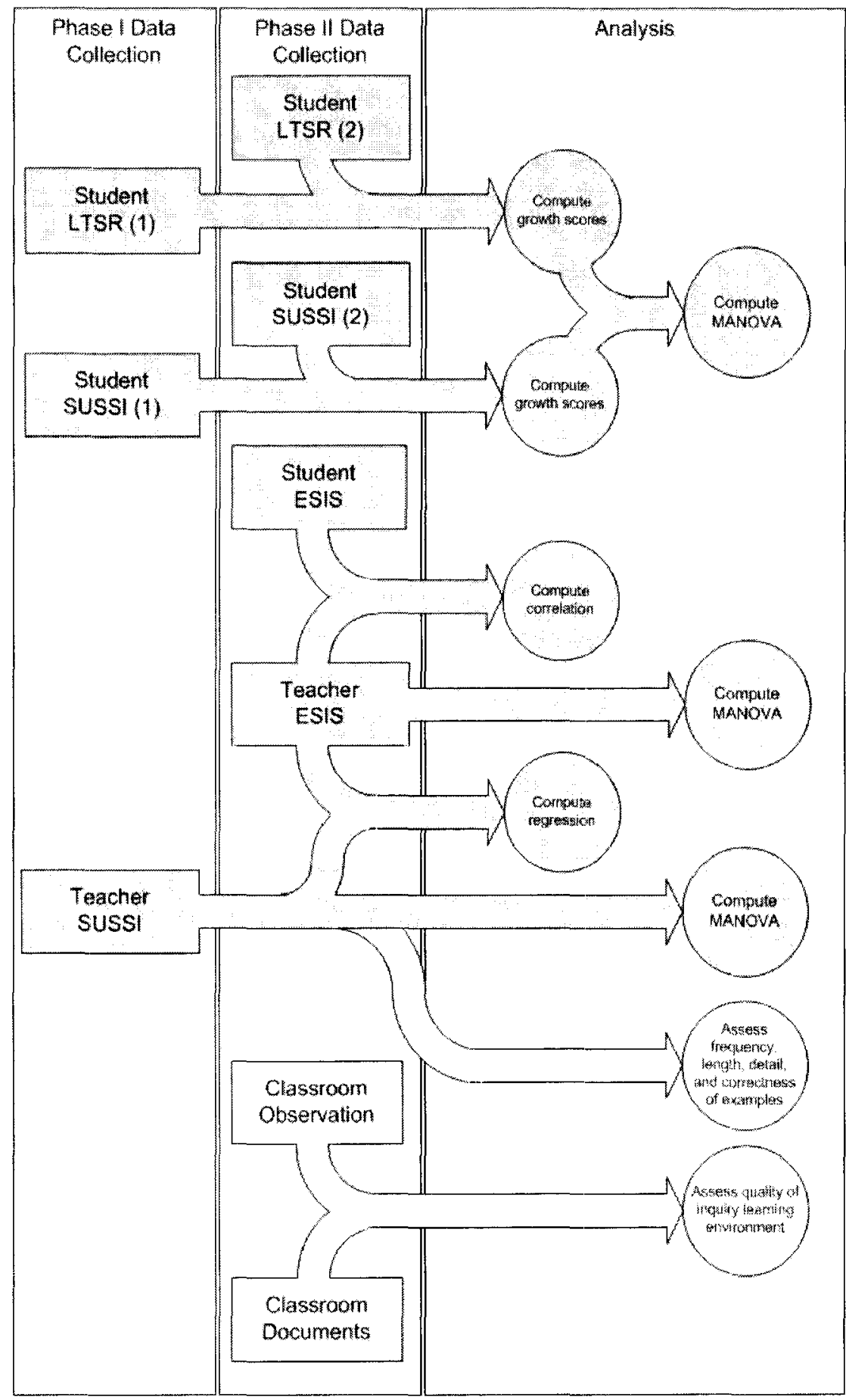

Figure 3.2. Phases of Data Collection and Analysis.

Solicitation of teacher participants. Electronic and mail contact information for Kentucky teachers was available through data requests to the Kentucky Educational Professional Standards Board (EPSB). All Kentucky AYA Science and EA Science 
NBCTs were invited to participate in this study. With three groups, if a moderate effect size could be attained, 42 to 54 subjects per group are necessary to detect differences between the groups. If a large effect size could be attained, 25 to 32 subjects per group would suffice (Stevens, 2002).

The non-NBCT sample was identified in two ways. Teachers in the same schools as NBCTs who agreed to participate were invited to participate in the study. Additional science teachers who taught in middle and high schools similar to those in which the NBCTs were teaching were also invited to participate. Institutional similarity was determined from school information obtained from the National Center for Education Statistics (NCES) describing school variables for teachers participating in this study (www.nces.ed.gov). These included location, SES status, size, and percentage of minority and migrant students.

Contact information of potential participants was gathered from the MAX Data System of the Kentucky Department of Education (http://kdemaxport3.kde.state.ky.us:7777/pls/portal_code.pkg_max.main?p_act=portal_c ode.pkg_max.sp_home_page) and through a data request to the KY EPSB. Teacher lists were cross-referenced between both data sources because the MAX Data System did not provide email addresses, and although the EPSB data did include email addresses, this source included several incorrect entries for each teacher. Early in the school year, teachers and their principals received electronic comnunication inviting them to participate in the study. Those who agreed to participate received instructions and instruments through the mail. 
Selection of student participants. The homogeneity of the student sample was increased by including only one class from each teacher in the study. This was important because in many high schools, after the sophomore year students may elect to enroll in advanced, standard, or lower track courses. Classes were selected that represented the most general population by excluding those that were either populated with extremely high functioning or low functioning students. To inform the selection, information was requested from participating teachers for all courses they taught: (a) Number of student, (b) number of English language learners, (c) number of students with an individual education plan, (d) course level (remedial, standard, honors), (e) grade level of students (number of each), and (f) ethnic group populations. Further artifact documentation was requested from those teachers being observed including lesson plans and student handouts related to the lesson observed.

\section{Participants}

Description of the teacher sample. Of 93 teachers who agreed to participate and for whom school and district-level permission was secured, 63 returned the first phase of documentation. Of those, 50 returned the second phase of documentation. Teachers participating in this study represented 38 Kentucky public school districts and 53 schools in the first phase, and 32 Kentucky public school districts and 45 schools in the second phase. Because the teacher demographic data was gathered during the second phase of data collection, teacher demographic variables other than National Board Certification status, years of experience, and course and grade level taught were only available for those who returned the second phase of assessments. Table 3.2 displays data describing teacher experience level, grade-level assignment, subject area, and school schedule for 
NBCTs and non-NBCTs participating in the first phase and second phase of data collection. In spite of attrition, the teacher sample remained surprisingly similar between the first and second phases of data collection. For these reasons, there appears to be no reason to suspect the presence of selection bias in the sample of teachers who completed both phases of data collection.

Table 3.2

Teacher Demographics Describing First and Second Phase Samples

\begin{tabular}{|c|c|c|c|c|}
\hline \multirow[b]{2}{*}{ Variable } & \multicolumn{2}{|c|}{ First Phase } & \multicolumn{2}{|c|}{ Second Phase } \\
\hline & $\begin{array}{c}\text { non-NBCT } \\
(\mathrm{n}=42)\end{array}$ & $\begin{array}{c}\text { NBCT } \\
(n=21)\end{array}$ & $\begin{array}{c}\text { non-NBCT } \\
(\mathrm{n}=31)\end{array}$ & $\begin{array}{c}\text { NBCT } \\
(n=19)\end{array}$ \\
\hline \multicolumn{5}{|c|}{ Experience Level } \\
\hline Novice $(0-5$ years $)$ & $15(36 \%)$ & -- & $12(39 \%)$ & -- \\
\hline Mid-Career ( $6-15$ years) & $14(33 \%)$ & $12(57 \%)$ & $8(26 \%)$ & $10(53 \%)$ \\
\hline Adv.-Career (16-32 years) & $13(31 \%)$ & $9(43 \%)$ & $11(36 \%)$ & $9(47 \%)$ \\
\hline \multicolumn{5}{|c|}{ Grade Taught } \\
\hline $6^{\text {th }}$ Grade & $8(19 \%)$ & -- & $6(19 \%)$ & -- \\
\hline $7^{\text {th }}$ Grade & $7(17 \%)$ & $5(24 \%)$ & $5(16 \%)$ & $5(26 \%)$ \\
\hline $8^{\text {th }}$ Grade & $4(10 \%)$ & $2(10 \%)$ & $4(13 \%)$ & $2(11 \%)$ \\
\hline $9^{\text {th }}$ Grade & $9(21 \%)$ & $6(29 \%)$ & $6(19 \%)$ & $4(21 \%)$ \\
\hline $10^{\text {th }}$ Grade & $7(17 \%)$ & $2(10 \%)$ & $6(19 \%)$ & $2(11 \%)$ \\
\hline $11^{\text {th }}$ Grade & $7(17 \%)$ & $6(29 \%)$ & $4(13 \%)$ & $6(32 \%)$ \\
\hline \multicolumn{5}{|c|}{ Subject Taught } \\
\hline Middle School Science & $19(45 \%)$ & $7(33 \%)$ & $15(48 \%)$ & $7(37 \%)$ \\
\hline Life Science & $6(14 \%)$ & $3(14 \%)$ & $4(13 \%)$ & $3(16 \%)$ \\
\hline Earth Science & $3(7 \%)$ & $1(5 \%)$ & $2(7 \%)$ & $1(5 \%)$ \\
\hline Physical Science & $13(31 \%)$ & $10(48 \%)$ & $10(32 \%)$ & $8(42 \%)$ \\
\hline \multicolumn{5}{|c|}{ School Schedule } \\
\hline Standard Schedule & $34(81 \%)$ & $14(67 \%)$ & $25(81 \%)$ & $14(74 \%)$ \\
\hline $4 \times 4$ Block Schedule & $7(17 \%)$ & $6(29 \%)$ & $6(19 \%)$ & $4(21 \%)$ \\
\hline AB Block Schedule & $1(2 \%)$ & $1(5 \%)$ & 0 & $1(5 \%)$ \\
\hline
\end{tabular}

Data describing participating teachers' non-education degree level and subject area were also collected as teachers' science education may explain their classroom inquiry practice. A teacher's non-education degree represents the highest degree in a science, technology, engineering, or mathematics (STEM) content area. It is used as a measure of teacher content knowledge. Table 3.3 displays teachers' self-report of their 
educational experience. One NBCT possessed a doctor of veterinary degree but because she was the only participant who possessed that level of training, she was included in the MA/MS category. Fifteen participants held only education-related degrees because middle school science teachers may practice in Kentucky without a non-education degree. Chi-square analysis of the frequency of non-education degree level between the NBCT and non-NBCT groups indicates that no significant difference in non-education degree level exists between the groups, $\chi^{2}(2)=0.544, p=0.762$. Chi-square analysis of the frequency of non-education degree level between middle and high school teachers did indicate the existence of significantly more degrees for high school teachers, however, $\chi^{2}(2)=21.278, p<0.001$. Further investigation indicated that when teachers were divided into four groups simultaneously describing NBCT status and middle/high school assignment, significant differences in the frequency of non-education degree remained, $\chi^{2}(2)=24.696, p<0.001$, with $50 \%$ of high school NBCTs and no middle school non-

NBCTS possessing Masters degrees.

Table 3.3

Teacher non-Education College Degree

\begin{tabular}{lcccc}
\hline & \multicolumn{2}{c}{ non-NBCT } & \multicolumn{2}{c}{ NBCT } \\
\hline Degree & $\mathrm{BA} / \mathrm{BS}$ & $\mathrm{MA} / \mathrm{MS}$ & $\mathrm{BA} / \mathrm{BS}$ & MA/MS \\
\hline Biology & $9^{\mathrm{c}}(29 \%)$ & 0 & $7^{\mathrm{a}}(37 \%)$ & $1^{\mathrm{b}}(5 \%)$ \\
Chemistry & $4^{\mathrm{c}}(13 \%)$ & $1(3 \%)$ & $1^{\mathrm{a}}(5 \%)$ & $1^{\mathrm{b}}(5 \%)$ \\
Physics/Engineering & $1(3 \%)$ & 0 & $2(13 \%)$ & 0 \\
Earth Science & 0 & $1(3 \%)$ & 0 & 0 \\
Animal Science & $2(6 \%)$ & 0 & $1(5 \%)$ & $2^{\mathrm{d}}(11 \%)$ \\
Health & $2(6 \%)$ & 0 & 0 & 0 \\
Psychology & $1(3 \%)$ & 0 & 0 & 0 \\
Mathematics & 0 & $1(3 \%)$ & 0 & 0 \\
None & $11(35 \%)$ & -- & $4(21 \%)$ & -- \\
\hline
\end{tabular}

Indicates percentage of total number of teachers in that category (non-NBCT/NBCT). a,b,c Indicates double major.

${ }^{\mathrm{d}}$ One participant holds a Doctor of Veterinary Medicine degree. 
All participating teachers and students were selected from Kentucky middle and high school classrooms. Several teachers possessed elementary school certifications, however. Table 3.4 presents frequency data for licensure level and teaching rank. In Kentucky a Rank III license is granted with initial certification at the Bachelor's degree level. Teachers must complete a master's degree or equivalent program within five years in order to receive Rank II credential. Rank II is required for teachers to maintain their teaching license. A Rank I credential is granted to a teacher with 30 hours of post-masters graduate work or NBCT status (www.kyepsb.ky.gov).

Table 3.4

Teacher Licensure Level

\begin{tabular}{lcc}
\hline & non-NBCT & NBCT \\
\hline \multicolumn{2}{c}{ School Level } \\
Elementary Level & 3 & \\
Middle School & 11 & 0 \\
High School & 17 & 6 \\
II & Rank & 13 \\
II & 7 & 0 \\
I & 17 & 0 \\
H & 7 & 19 \\
\hline
\end{tabular}

In Kentucky, certification Rank I is granted with NBCT status as well as by 30 hours of post-master's degree graduate credit.

Figure 3.3 displays histograms of teacher experience levels (Novice, Mid-Career, Advanced-Career) paneled by NBCT status. This information was important because teacher experience level may have explained classroom practice. In order to compare experience levels between NBCT and non-NBCT groups, the teacher sample was divided into three groups: Those with five or fewer years teaching experience (Novice), those with five to fifteen years experience (Mid-Career), and those with sixteen to thirty-two years experience (Advanced-Career). Table 3.5 indicates that there was substantial similarity between the levels of experience of the comparable NBCT and non-NBCT 
groups. No NBCTs were represented in the novice experience level because in order to be eligible to pursue National Board Certification, a teacher must have at least three years' teaching experience. Independent t-tests of years teaching experience between the NBCT and non-NBCT groups indicated no significant differences in experience level for Mid-Career teachers $\mathrm{t}(16)=0.076, \mathrm{p}=0.940$ or for Advanced-Career teachers $\mathrm{t}(18)=-$ $0.086, \mathrm{p}=0.932$. No comparison was available for Novice teachers because NBCTs were not represented in this group.

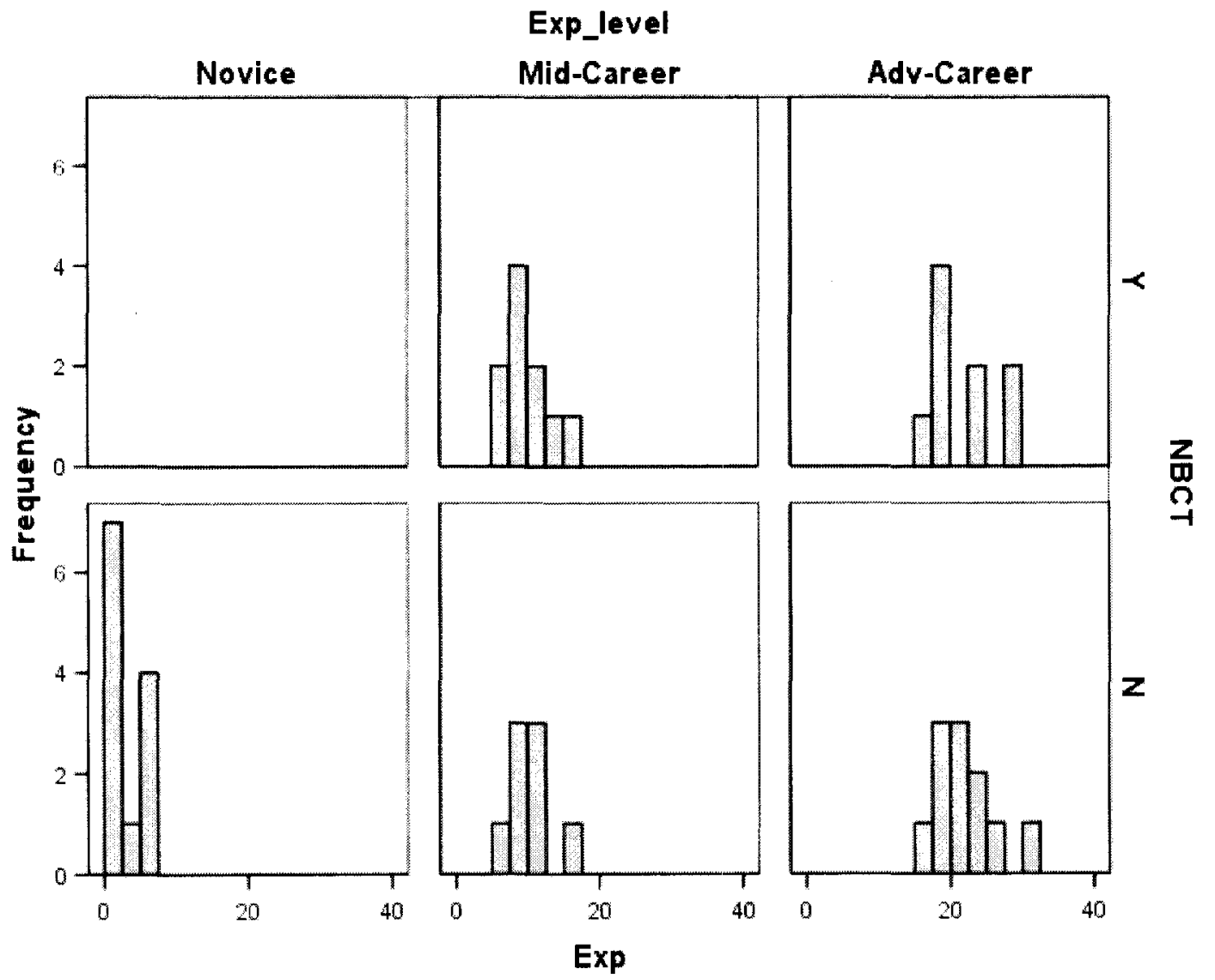

Figure 3.3

Experience of Second Phase Teacher Sample Paneled by NBCT and Experience Level 
Table 3.5

Description of Years Experience for Teacher Sample by Experience Level

\begin{tabular}{lcccc}
\hline & \multicolumn{2}{c}{ non-NBCT } & \multicolumn{2}{c}{ NBCT } \\
\cline { 2 - 5 } Experience Level & $\mathrm{n}$ & Mean (SD) & $\mathrm{n}$ & Mean (SD) \\
\hline 0-5 years & 12 & $2.67(2.015)$ & -- & - \\
5-15 years & 8 & $10.00(2.828)$ & 10 & $10.10(2.726)$ \\
16-32 years & 11 & $21.73(4.292)$ & 9 & $21.56(4.613)$ \\
\hline
\end{tabular}

Description of the student sample. Description of the student sample originated in data from teacher enrollment forms and the classroom information sheets completed during the second phase of assessments. Data describing class ethnicity were difficult to analyze due to participant data-entry idiosyncrasies, but sufficient data were present to enable description of classes as majority white with small percentages of African American and Latin American students. Both student assessments have been shown to be developmentally linked. This means that as students mature they tend to score higher of the assessments. Therefore, accounting for the age-level of students in this study was necessary. Table 3.6 displays the grade level of students participating in this study organized by the NBCT status of their teacher and by phase of data collection.

Table 3.6

Number of Students by Grade Level

\begin{tabular}{lcccc}
\hline & \multicolumn{2}{c}{ First Phase $(\mathrm{n}=1520)$} & \multicolumn{2}{c}{ Second Phase $(\mathrm{n}=1181)$} \\
Grade Level & non-NBCT & NBCT & non-NBCT & NBCT \\
\hline $6^{\text {th }}$ & 207 & 0 & 141 & 0 \\
$7^{\text {th }}$ & 179 & 116 & 127 & 108 \\
$8^{\text {th }}$ & 88 & 55 & 106 & 51 \\
$9^{\text {th }}$ & 203 & 164 & 144 & 100 \\
$10^{\text {th }}$ & 162 & 45 & 134 & 42 \\
$11^{\text {th }}$ & 154 & 147 & 82 & 146 \\
Sub-Total & 993 & 527 & 734 & 447 \\
\hline
\end{tabular}

Table 3.7 displays data describing the average NBCT and non-NBCT class size. It is clear that any differences in average class size appear to favor non-NBCTs. Table 3.8 
displays teacher perception of their students' achievennent level compared to that of students in the entire school.

Table 3.7

Average Class Size

\begin{tabular}{ccc}
\hline Class Size & non-NBCT & NBCT \\
Mean (SD) & Mean (SD) \\
\hline $6^{\text {th }}$ Grade & $26.63(2.669)$ & -- \\
$7^{\text {th }}$ Grade & $27.14(3.132)$ & $25.40(2.074)$ \\
$8^{\text {th }}$ Grade & $27.75(3.862)$ & $27.50(3.536)$ \\
$9^{\text {th }}$ Grade & $24.22(2.949)$ & $29.67(2.805)$ \\
$10^{\text {th }}$ Grade & $25.29(5.880)$ & $22.50(2.121)$ \\
$11^{\text {th }}$ Grade & $22.14(5.367)$ & $26.33(3.386)$ \\
\hline
\end{tabular}

Table 3.8

Teacher Perception of Participating Class' Achievement ${ }^{\text {a }}$

\begin{tabular}{lcc}
\hline Rating & non-NBCT $(\mathrm{n}=31)$ & NBCT $(\mathrm{n}=19)$ \\
\hline Blank & 1 & 1 \\
Wide Range & $6(19 \%)$ & $1(5 \%)$ \\
Low & $6(19 \%)$ & $5(26 \%)$ \\
Average & $11(35 \%)$ & $6(32 \%)$ \\
High & $7(23 \%)$ & $6(32 \%)$ \\
\hline
\end{tabular}

${ }^{\text {a }}$ Compared to other students in the teacher's school.

Because much of the variance in student achievement is between-school variation, data were collected from the National Center for Education Statistics (NCES) describing school variables for teachers participating in this study (location, SES status, size, and percentage of minority and migrant students) (www.nces.ed.gov). Table 3.9 displays these school-level demographic variables.

Table 3.9

School-level Demographic Variables

\begin{tabular}{lcc}
\hline Variable & non-NBCT & NBCT \\
\hline \multicolumn{3}{c}{ Locale } \\
Rural, Far from Town & schools) & \\
Rural, Near Town & 6 & 5 \\
Small Town & 11 & 6 \\
Urban Fringe of Mid-Size City & 3 & 4 \\
Mid-Size Central City & 0 & 1 \\
Urban Fringe of Large City & 2 & 0 \\
& 7 & 2
\end{tabular}


Large Central City 221

Magnet School

School Description

Title 1 School

$1 \quad 1$

School Demographics

Number of Students [Mean (SD)] $\quad 876(387) \quad 1033(502)$

Number of Teachers

$50(20)$

$57(25)$

Student:Teacher Ratio

$17.5(1.76)$

$17.9(1.90)$

Number of Migrant Students

$8(13)$

$11(16)$

Percentage Minority

$8.23(8.8)$

$7.23(9.30)$

\section{Summary}

Grade-level certification, teaching experience, and non-education degree were all identified as potential counterfactuals to the research hypotheses. The analysis presented in this section indicated that NBCT and non-NBCT groups participating in this study were substantially similar on these variables, however. Data reported in these tables indicate there was substantial similarity among the students and schools of the NBCTs and their non-NBCT counterparts participating in this study. The reduction in the size of teacher and student samples from phase one to phase two of data collection created a situation in which approximately $26 \%$ of students involved in the first phase of data collection were missing the second phase of data. This affected those analyses in which change in student knowledge and understanding were measured. For this reason, only those teachers and students who completed both phases of data collection were included in the analysis. The significant similarity between first and second phase teacher and student demographics diminishes the chances of selection bias affecting the reliability of results.

\section{Variables and Measures}

In addition to data collected relating directly to the research questions, other data were collected addressing potential counterfactuals explaining trends in the data. Teacher 
professional qualities shown to impact student achievement were collected. For example, state licensure status, post-secondary degrees granted, number of years teaching experience (Cavalluzzo, 2004) and number of hours of graduate-level science coursework (Monk \& King-Rice, 1994) have been shown to effect teacher effectiveness while research experience (Schwartz \& Lederman, 2002) has been shown to have an impact on teacher understanding of nature of science. Additionally, teacher perceptions of school climate and factors that limit their inquiry practice, along with demographic data describing student dispositions and ability, and general teacher pedagogical practice were collected on a demographic sheet attached to the Elementary Science Inquiry Study (ESIS) teacher instrument (See Appendix B).

Question 1: How do National Board Certified science teachers' knowledge of the nature of science differ from that of their non-certified counterparts?

One factor cited in the research literature impacting teachers' science inquiry teaching ability is their own understanding of the nature of science inquiry. For example, in a qualitative case study Schwartz and Lederman (2002) found that teachers who were experienced with science inquiry provided better instruction in science inquiry. Effective inquiry instruction develops understanding of the nature of science as well as science inquiry skills (Von Secker \& Lissitz, 1999). The first question addresses differences in teacher NOS understanding.

\section{Instrumentation}

Significant controversy exists concerning the validity and reliability of measurements of NOS understanding (Abd-El-Khalick, Bell, \& Lederman, 1998). For this reason, careful consideration was paid to the selection of NOS understanding 
instrumentation and interpretation of the data gathered from application of those instruments. Well known instruments include the Views on Nature of Science (VNOS) (Lederman, Abd-El-Khalick, Bell, \& Schwartz, 2002) and the Views on ScienceTechnology-Society (VOSTS) instrument (Aikenhead \& Ryan, 1992). The former is an interview protocol, and the latter is a multiple choice instrument consisting of items and distractors derived from student misconceptions regarding NOS. The complex nature of these instruments limited their suitability for this study however.

To measure the understanding of the NOS that formed the foundation for science inquiry teaching, teachers completed the Student Understanding of Science and Science Inquiry (SUSSI) instrument (See Appendix C) (Liang, et al., 2006). The SUSSI instrument was developed using a multi-stage process. International science standards documents were examined for NOS and science inquiry concepts. Seven standards were identified: (a) Tentativeness of scientific knowledge, (b) the difference between observations and inferences, (c) the presence of subjectivity and objectivity in science, (d) the role of creativity and rationality in science, (e) the social and cultural embeddedness of science, (f) the difference between scientific theories and laws, and ( $g$ ) the role of scientific methods (Liang, et al., 2006).

Items were written based on the VOSTS instrument (Aikenhead \& Ryan, 1992) and VNOS (Lederman, Abd-El-Khalick, Bell \& Schwartz, 2002). Items were then vetted by an international panel of experts. The instrument was revised based on the results of two phases of pilot study. The instrument consists of 24 items describing six domains of understanding: (a) Observation and inferences, (b) change in scientific theories, (c) difference between scientific laws and theories, (d) social and cultural influence on 
science, (e) role of imagination and creativity in scientific investigations, and (f) methodology of scientific investigations. Subjects in several countries were asked to rate their agreement with each statement on a five anchor Likert-type scale from "strongly disagree" to "strongly agree" and cite examples to justify answers. Results suggested that the SUSSI instrument has validity not only assessing NOS concepts but may do so across cultural boundaries (Liang, et al., 2006).

Analysis

In order to discern differences in NOS understanding between the NBCT and nonNBCT samples in this study, independent $t$-tests were used to compare teachers on each of the six SUSSI sub-scales as well as the total score. In order to assess the impact of potential counterfactuals, separate MANOVAs were computed to compare teachers on experience level, non-education degree, teaching rank., and middle/high school assignment.

Question 2: How do the frequencies of student science inquiry behaviors supported by learning environments created by NBCTs differ from those created by their non-certified counterparts?

Dependent teacher variables in this study were frequencies of teacher science inquiry instructional behaviors. The definition of these behaviors was based on the seven NSES high school science inquiry standards (NRC, 1996). The behaviors were operationalized as frequency ratings of teacher and student behaviors such as "use data to construct a reasonable explanation" and "choose appropriate tools for an investigation." 


\section{Instrumentation}

For this study, the classroom inquiry environment teachers created was assessed using the Elementary Science Inquiry Survey (ESIS) (Dunbar, 2002). This survey consists of 29 items describing student and teacher behaviors indicative of strong or weak inquiry instruction. The Likert-type scale was a frequency rating on a scale with six levels ranging from "never" to "more that 5 times a week." The ESIS instrument was originally developed to assess the classroom inquiry practice of fourth and fifth grade teachers but was suitable for middle and high school teachers because the inquiry behaviors it assessed are generally related to effective science inquiry pedagogy and not developmentally linked. As part of the validation process, Dunbar (2002) factor analyzed the results using varimax rotation and Kaiser-normalization to determine their structure. The factor analysis provided a six factor solution explaining $66 \%$ of the variance in responses, but the researcher then forced a five factor solution to match the number of "abilities necessary to do scientific inquiry" in the NSES. This solution explained $54 \%$ of the variance (Dunbar, 2002). Dunbar's factor structure was used to collapse results from this study.

For the purposes of this study, teachers completed an adapted version of Dunbar's original instrument. Adaptations consisted of re-anchoring items following Dunbar's recommendations after his validation of the instrument (Dunbar, 2002), replacing the demographic information section with one representative of this population of Kentucky middle and high school teachers. To strengthen the construct validity of the ESIS, a panel of experts rated the items constituting the instrument according to the NSES high school 
science inquiry standards. No changes to the instrument were indicated as the result of this review.

\section{Observation protocols}

Observations of classroom inquiry teaching were obtained to triangulate ESIS results describing classroom science inquiry practices. Observations were made of a randomly selected sub-sample of those teachers who agreed to be observed. This subsample consisted of five middle school and three high school teachers which constituted $16 \%$ of the total teacher sample. Classrooms were observed using both low and high inference protocols.

Low inference observations were structured using the Collaboratives for Excellence in Teacher Preparation (CETP) Classroom Observation Protocol (COP) (Lawrenz, Huffman, \& Appleldoorn, 2002), which organized the tabulation of the frequency of instructional activities, the level of student engagement, and cognitive activity present in the classroom (See Appendix D). Low inference observations were used to assess basic teaching qualities and learning environment characteristics including type of teacher behavior, level of student engagement, and level of student cognitive activity. The CETP Core Evaluation Classroom Observation Protocol (COP) was developed to evaluate of the effect of the CETP (Lawrenz, Huffman, \& Appleldoorn, 2002), a professional development program started by the NSF in 1992. The portion of the instrument that was used in this study included timed observations of teacher and student behaviors.

High inference observations were structured by the Reform Teaching Observation Protocol (RTOP), an instrument designed to assess reform teaching practices in K-16 
mathematics and science classes. This protocol was developed at Arizona State University to measure reformed classroom teaching. Reformed teaching is characterized by several qualities: (a) It incorporates students' preconceptions; (b) it recognizes that learning progresses from concrete to abstract; and (c) learning does not occur in isolation, but in community of learners; and (d) it causes students to engage in activities that cause them to reflect on their work (Piburn \& Sawada, 2000).

The RTOP instrument consists of 25 items distributed into three groups: Lesson design and development, content, and classroom culture. The second and third groups are divided into two groups each, creating five subscales: (a) Lesson design and implementation, (b) propositional knowledge, (c) procedural knowledge, (d) communicative interactions, and (e) student/teacher relationships (See Appendix E) (Piburn \& Sawada, 2000). The RTOP provides a total score on a scale from 0 to 100 that may be used to rate classroom environment, but it also enables the collection of evidence gathered in response to prompts, analyzed qualitatively.

The RTOP is a structured observation protocol requiring training to enhance the reliability of observations and inferences. Prior to observation, the researcher was trained to use the RTOP using the online training materials (http://PhysicsEd.BuffaloState.Edu). Training consisted of taking notes while viewing three short video segments depicting classroom instruction. Observation notes were then used to rate the observation on the standard RTOP form according to the five subscales listed earlier. After this trial rating was complete, it was compared to the standard rating archived on the website. The researcher noted differences between his rating and the standard rating. The researcher then reviewed the video material noting where the discrepancies had occurred and 
rectifying them. The researcher then repeated this process until his assessment of the lesson approximated the standard assessment available with the training materials. Establishing Instrument Validity

Triangulation. Self-reported data may be biased (Shadish, Cook \& Campbell, 2002). Teacher perceptions of the learning environment they created may not have accurately portrayed the environment as it was perceived by students. Therefore, in order to verify teacher perceptions of the science inquiry activity occurring in the classroom, students also completed the ESIS instrument.

Substantial similarity between student and teacher responses would correspond to a large probability that both teacher and student survey responses reflected observations of the same setting and would indicate that teacher responses to the ESIS survey were adequate measures of their classroom inquiry behavior. Lack of correlation between teacher and student responses would limit the confidence with which the teacher responses could be interpreted.

Correlation between teacher and student ESIS responses. In order to compare the two sets of survey responses, inter-rater analysis was the appropriate measure of fidelity because this was a situation in which multiple individuals were rating the same characteristic. To do this, each teacher's response to each of the twenty-nine questions was correlated with the mean response to that question from students in that teacher's class. Correlations between teacher and student responses were aggregated according to significance level. Correlations with significance less than 0.70 had greater than $30 \%$ probability that they were in agreement. Correlations with significance less than 0.20 had greater than $80 \%$ probability that they were in agreement. Table 3.10 displays the 
percentage of teacher and student correlations with three levels of statistical significance, aggregated by teacher grade-level assignment, NBCT status, and experience level. This analysis indicated insufficient levels of agreement between teacher and student ratings of inquiry-related classroom behaviors, implying further analysis was warranted.

Table 3.10

Teacher/Student ESIS Correlations by Grade, NBCT Status, and Experience

\begin{tabular}{lcccc}
\hline \multirow{2}{*}{ Characteristic } & \multicolumn{4}{c}{ Percent Teacher-Student Correlations } \\
\cline { 2 - 5 } & $\mathrm{N}$ & $1>\mathrm{p}>0.7$ & $0.7>\mathrm{p}>0.2$ & $\mathrm{p}<0.2$ \\
\hline $6^{\text {th }}$ & 6 & Grade & $17 \%$ & $50 \%$ \\
$7^{\text {th }}$ & 10 & $10 \%$ & $50 \%$ & $40 \%$ \\
$8^{\text {th }}$ & 6 & $33 \%$ & $0 \%$ & $67 \%$ \\
$9^{\text {th }}$ & 10 & $10 \%$ & $30 \%$ & $60 \%$ \\
$10^{\text {th }}$ & 8 & $13 \%$ & $25 \%$ & $63 \%$ \\
$11^{\text {th }}$ & 10 & $20 \%$ & $20 \%$ & $60 \%$ \\
\multicolumn{5}{c}{ NBCT Status } \\
non-NBCT & 31 & $24 \%$ & $26 \%$ & $50 \%$ \\
NBCT & 19 & $11 \%$ & $21 \%$ & $68 \%$ \\
5 years or fewer & 11 & $28 \%$ & $27 \%$ & $45 \%$ \\
Between 5 and 16 years & 19 & $10 \%$ & $32 \%$ & $58 \%$ \\
16 years or more & 20 & $15 \%$ & $35 \%$ & $50 \%$ \\
\hline Overall & 50 & $18 \%$ & $26 \%$ & $56 \%$ \\
\hline \multicolumn{5}{c}{}
\end{tabular}

Internal consistency reliability. Analysis continued with examination of the internal consistency of each set of responses to determine if either seemed to be more stable measures of classroom inquiry environment. The mean total ESIS-score for all teachers was 62.48 with a standard deviation of 14.352 . The mean total ESIS-score for all students was 72.30 with a standard deviation of 14.96 . The internal consistency (Cronbach's alpha) of the ESIS instrument based on 50 teacher cases was 0.820 . The internal consistency of the student responses however, was 0.937 . These results indicated that student responses to the ESIS may have been a more stable and trustworthy measure of classroom inquiry behaviors than teacher responses. Table 3.11 displays how the 
instrument would perform if each of the items were deleted from the survey. Poorly

performing items would decrease the internal consistency of the instrument as a whole,

increasing the variance of the scale and thus decreasing the reliability (Cronbach's alpha).

Although some items appeared to have contributed variance to the total score, no item

significantly adversely impacted the performance of the instrument as a whole.

Table 3.11

Reliability Data for ESIS Items 1-29

\begin{tabular}{lcccccc}
\hline & \multicolumn{3}{c}{ Teacher Responses } & \multicolumn{3}{c}{ Student Responses } \\
\cline { 2 - 7 } & Scale & Scale & Cronbach's & Scale & Scale & Cronbach's \\
Item & Mean if & Variance if & Alpha if & Mean if & Variance if & Alpha if \\
\hline 1 & 60.54 & Deleted & Deleted & Deleted & Deleted & Deleted \\
2 & 59.96 & 200.611 & 0.824 & 67.56 & 603.750 & 0.937 \\
3 & 59.96 & 203.876 & 0.821 & 67.18 & 600.614 & 0.936 \\
4 & 60.28 & 199.798 & 0.817 & 67.43 & 600.315 & 0.936 \\
5 & 60.04 & 203.141 & 0.821 & 66.99 & 599.171 & 0.936 \\
6 & 60.34 & 178.025 & 0.800 & 67.16 & 574.580 & 0.936 \\
7 & 60.66 & 187.617 & 0.811 & 66.92 & 581.151 & 0.936 \\
8 & 60.02 & 184.306 & 0.805 & 67.18 & 579.292 & 0.934 \\
9 & 60.90 & 191.643 & 0.811 & 67.17 & 579.124 & 0.935 \\
10 & 61.00 & 193.959 & 0.817 & 67.04 & 579.422 & 0.935 \\
11 & 60.06 & 186.588 & 0.807 & 67.09 & 576.373 & 0.934 \\
12 & 59.70 & 190.622 & 0.815 & 67.18 & 576.612 & 0.934 \\
13 & 60.52 & 181.928 & 0.802 & 67.10 & 577.283 & 0.934 \\
14 & 61.48 & 192.949 & 0.812 & 67.35 & 574.864 & 0.934 \\
15 & 60.72 & 187.920 & 0.808 & 66.90 & 577.397 & 0.935 \\
16 & 60.54 & 197.764 & 0.820 & 67.54 & 582.404 & 0.935 \\
17 & 61.20 & 204.204 & 0.833 & 67.73 & 585.244 & 0.936 \\
18 & 60.96 & 196.774 & 0.817 & 67.31 & 579.661 & 0.934 \\
19 & 60.74 & 194.645 & 0.818 & 67.58 & 587.468 & 0.936 \\
20 & 61.12 & 205.659 & 0.829 & 67.31 & 596.299 & 0.939 \\
21 & 59.80 & 189.061 & 0.813 & 67.06 & 575.407 & 0.934 \\
22 & 60.34 & 182.637 & 0.807 & 67.20 & 575.526 & 0.934 \\
23 & 60.38 & 181.261 & 0.801 & 67.04 & 572.909 & 0.934 \\
24 & 60.32 & 201.242 & 0.826 & 66.91 & 580.276 & 0.935 \\
25 & 58.54 & 191.192 & 0.813 & 66.55 & 578.887 & 0.935 \\
26 & 58.48 & 193.847 & 0.814 & 66.87 & 573.301 & 0.935 \\
27 & 59.30 & 187.153 & 0.812 & 67.02 & 572.871 & 0.934 \\
28 & 61.44 & 200.333 & 0.821 & 67.21 & 575.899 & 0.934 \\
29 & 60.10 & 189.480 & 0.815 & 66.95 & 572.880 & 0.934 \\
\hline & & & & & &
\end{tabular}


Comparison of teacher and student ESIS response trends. Another comparison between teacher and student responses to the ESIS survey items was the creation of a chart of mean teacher and student responses to each item. If the two sources of data tracked in the same way-that is, increasing and decreasing the same way from item to item - then this analysis would add validity to those measures of the classroom inquiry behaviors. Those items for which the teachers reported a relatively high value while the students reported a relatively low value would merit examination. Figure 3.4 displays the mean teacher and student responses to the 29 ESIS classroom inquiry survey items. Items $7,8,9,10,12,13,16,19,20$, and 26 potentially merited further inspection because teacher and student trends were in opposite directions for these, while for the other items the increasing or decreasing trends matched.

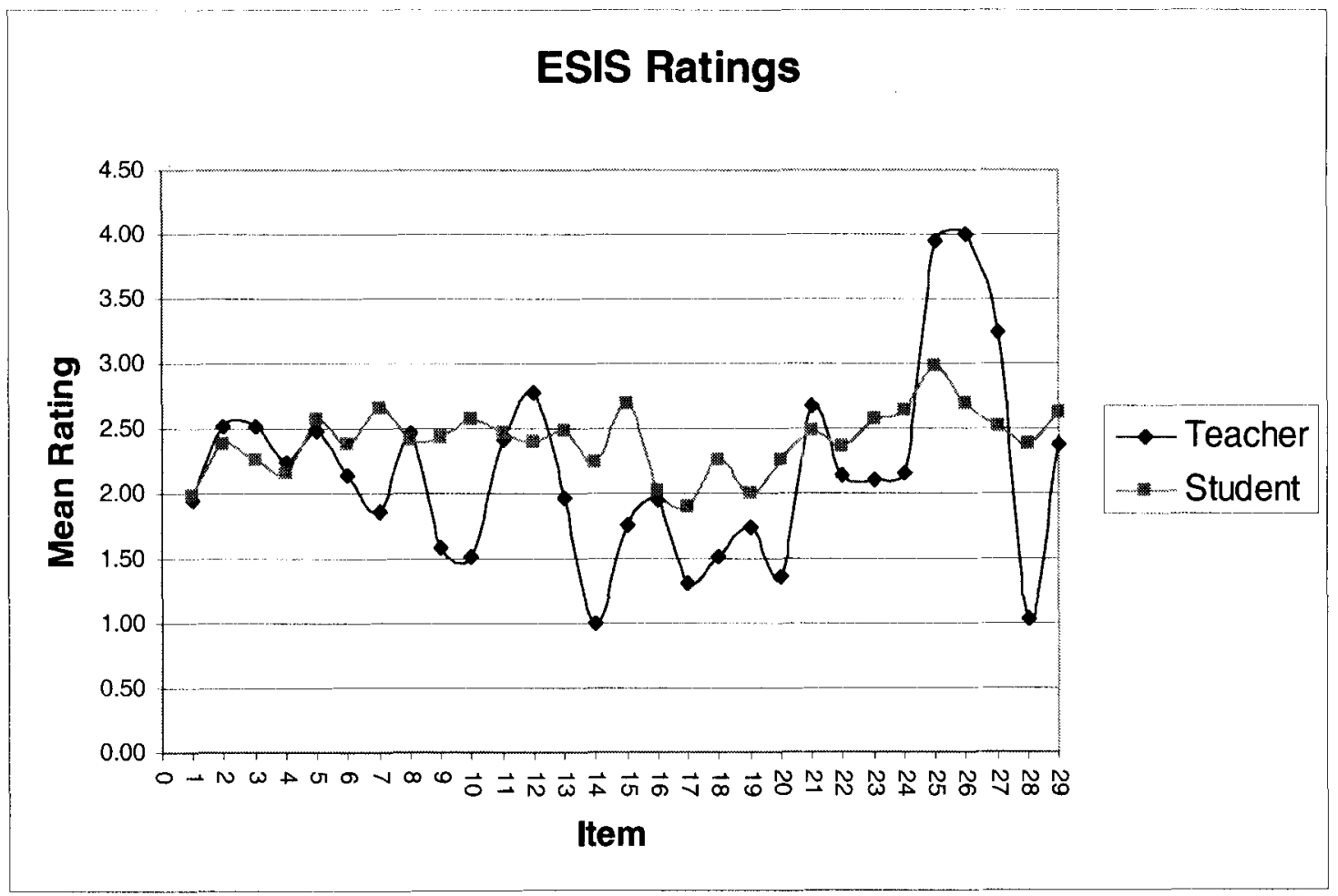

Figure 3.4. Comparison of Mean Teacher and Student Responses to ESIS Classroom Inquiry Questions 
Factor analysis. The 29 ESIS questions were intended to reflect the five inquiry behaviors described in the NSES (Dunbar, 2002). Dunbar established a factor structure during the validation phase of instrument development using a sample of $186 \mathrm{New}$ Mexico fourth and fifth grade teachers. Too few teachers participated in this study, however, to enable determination of the factor structure organizing these responses from these middle and high school teachers.

Instead, in this study, student responses were factor analyzed and the structure that emerged was compared against the original structure proposed by Dunbar (Table 3.12). When reordered to match Dunbar's factors, the majority of student responses replicated the factor structure found in the Dunbar's original study. For example, Dunbar interpreted the factor explaining the greatest proportion of the variance (Items 6, 9, 11, $12,13,14,15,16,18,19,21,22,23,27$, and 29) to correspond to Investigation. Student responses in the current study to ten of those fifteen items collapsed into the same factor, but only explained about seven percent of the variance. A reasonable interpretation of this reordering of factors is that although students participating in this study and Dunbar's teachers responded in similar ways to these classroom inquiry behaviors, differing perceptions and priorities cause the corresponding factors to explain different amounts of the variance in the responses of the two groups.

Table 3.12

ESIS Item Factor Assignment

\begin{tabular}{llclc}
\hline Factor & $\begin{array}{l}\text { Original } \\
\text { Structure }\end{array}$ & $\begin{array}{c}\text { \%Variance } \\
\text { Explained }\end{array}$ & $\begin{array}{l}\text { Study Student } \\
\text { Structure }\end{array}$ & $\begin{array}{c}\text { \%Variance } \\
\text { Explained }\end{array}$ \\
\hline 1 Investigation & $6,9,11,12$, & $40.26 \%$ & $6,7,8,9,10$, & $6.65 \%$ \\
& $13,14,15,16$, & & $11,12,13,14$, & \\
& $18,19,21,22$, & & 15 & \\
& $23,27,29$ & & & \\
2 Use of data for & $2,3,4,5,6,8$, & $4.76 \%$ & $1,2,3,4,5$ & $5.54 \%$ \\
explanation & $9,13,16$ & & &
\end{tabular}


3 Science dialogue

$21,22,23,25, \quad 4.35 \%$

$21,22,23,24$,

$37.26 \%$ 26,27

$25,26,27,28$, 29

4 Approach to science

7,10

17,24

$2.90 \%$

$16,17,18,19$

$3.86 \%$

5 Inquiry duration and

$2.26 \%$

20

$3.52 \%$

questions

The internal reliability of student responses in this study and the similarity of the factor structure displayed by student responses with Dunbar's original structure provided justification for collapsing responses into Dunbar's five factors to facilitate analysi, and to use student responses (rather than teacher responses) as a measure of classroom inquiry behaviors. ESIS factor sub-scores were tabulated following the factor structure displayed by students in this study.

Analysis

MANOVA. Because important differences exist between middle and high schools, teacher and student data were disaggregated into middle school and high school groups. Multivariate analysis of variance (MANOVA) was completed separately for middle and high school groups using NBCT status, teacher experience level, and non-education degree as independent variables, and student responses to the first four ESIS factors as dependent variables. The fifth factor was eliminated because it corresponded to a single negatively worded question.

Observation. The researcher interviewed participating teachers before and after observations, gathering background and context information regarding the observed lesson, class activities, classroom context, and school climate. The low-inference observation protocol structured observations of teacher behavior, student engagement, and student cognitive activity every five minutes (see Appendix D). Analysis of this data 
consisted of tabulating frequencies of instructional behaviors and counting the extent of student engagement and cognitive activity.

Simultaneously with these low-inference observations, the researcher scripted notes describing the physical environment of the room, along with description of teacher and student activity, and discourse present as the lesson progressed. Field notes consisted of a running record of descriptive data and observer comments recording researcher emotional reactions, analytic insights, and questions for further analysis (Rossman \& Rallis, 2003). Within a couple days of the observation the field notes were transcribed into a computer file. At this point, the notes were augmented to provide thick description (Geertz, 1973) necessary to interpret and understand the activities, discourse and social relationships that were observed during the lesson (Denzin, 1994). Because qualitative data were being analyzed to triangulate inferences drawn from other data sources and analysis they were coded according to the five categories representing sub-scales of the RTOP observation protocol: (a) Lesson design and implementation, (b) propositional knowledge, (c) procedural knowledge, (d) communicative interactions, and (e) student/teacher relationships. Interactions were coded as implying strength or weakness for each of the five RTOP codes. Coded observation notes were used as a data source from which to complete the RTOP classroom inquiry inventory and interpret its results.

Documentary analysis. Teachers who were observed were invited to submit the set of documents supporting the science unit they selected for observation. Requested documents included: unit and lesson plans, worksheets, assignment sheets, assessment instruments, and student work samples. These documents were analyzed for reformed teaching qualities including: Levels of student communication and student choice; the 
cognitive level of teacher questions; and classroom policies supportive of high levels of student engagement (Piburn \& Sawada, 2000).

Question 3: What is the relationship between the frequency of students' science inquiry behaviors and their science reasoning and understanding of the nature of science?

Good teaching may be differentiated from effective teaching (Fenstermacher, 2005). A teacher may enact good teaching practice without effectively changing student understanding or behavior. Effective teachers recognize the myriad forces impacting students' lives and learning, and adapt their practice in ways to promote individual student achievement. For this reason, connecting teacher practice with student achievement is important—especially when accomplished teachers were being studied. This study was designed to discover if an enhanced science inquiry learning environment created by participating teachers improved students' science reasoning skills and NOS comprehension.

\section{Variables}

Independent variables. Students' demographic and academic variables describing their perception of the learning environment in their classrooms (ESIS survey) and their science reasoning (LTSR) and NOS understanding (SUSSI) were collected. In order to protect student identities, teachers recorded student information on a pre-coded sheet (see Appendix F) and then distributed test packets with the corresponding code to the appropriate student. Teachers recorded classroom-level demographic information as well.

Dependent variables. For this study, student science inquiry knowledge was conceptualized as a combination of science reasoning and understanding of the nature of science. Science reasoning skills were operationalized as understanding (a) control of 
variables, (b) difference between observations and inferences, and (c) experimental design (Lawson, 2000). NOS comprehension was operationalized by the SUSSI instrument (Liang, et al, 2006) as described earlier in this chapter.

\section{Instrumentation}

In addition to the SUSSI instrument assessing NOS understanding, Lawson's Test of Scientific Reasoning (LTSR) (Lawson, 2000) assesses reasoning skills associated with science inquiry. Lawson's assessment was initially created in 1978 to test formal operational thinking (its initial title was "Lawson's Classroom Test of Formal Reasoning") (Lawson, 1978). The assessment's first version included fifteen questions covering topics including proportional reasoning, control of variables, combinatorial reasoning, and probability. The LTSR received construct validity through reference to developmental cognitive theory. Other forms of validity were assessed through comparison of test scores with interview and performance tasks, and through factor analysis. The current version of the assessment (Lawson, 2000) contains twenty-four questions that assess scientific reasoning more extensively than previous versions (See Appendix $\mathrm{G}$ ). The current form of the assessment received validity through the series of studies in which it was used in the intervening period of time and from the progression of research hypotheses and consequential structural changes to the instrument (Lawson, personal communication, July 22, 2006). Table 3.13 categorizes the questions with the construct they were intended to measure.

Table 3.13

\begin{tabular}{ll}
\multicolumn{2}{l}{ LTSR Question- } \\
\hline Question & Construct Assignment \\
\hline 1,2 & Construct \\
3,4 & Displaced volume \\
$5-8$ & Proportional reasoning
\end{tabular}




\begin{tabular}{lll}
9,10 & Control of variables \\
$11-14 ; 19-20$ & Observation and inference \\
$15-18$ & Probability \\
$21-24$ & Experimental design \\
\hline
\end{tabular}

MANOVA. MANOVA was determined to be the appropriate test to detect differences among three or more groups on more than one interval-level dependent variable (Stevens, 2002). Differences in student science reasoning and NOS understanding related to teacher characteristics were uncovered using separate MANOVAs to compare student achievement scores for middle and high school groups and teacher NBCT status, experience level, and non-education degree.

In order to utilize the LTSR and SUSSI data in these quantitative analyses, responses were assigned numerical values. For the purposes of these analyses the ordinal values assigned to each measure were assumed to represent underlying interval-level constructs. The LTSR is a two-tier multiple choice test in which each pair of questions first targets a basic concept, and then identifies if the student answered correctly for the right reason. Students only received credit for an answer if target-concept answer and its follow-up answer were both correct. For this reason, in this study the 24-question LTSR scaled from 0 to 12 points. The SUSSI responses were coded from "SD—strongly disagree" through "SA—strongly agree." The prompts describe either "more informed views" or "more naïve views." Prompts were translated with +2 corresponding to the "most informed view" through -2 corresponding to the "most naïve view." 0 was assigned to the response indicating no opinion. Aggregate scores were calculated for each of the sub-categories of the LTSR and SUSSI instruments and used as separate dependent variables in the analysis. 
Regression. The sub-scores defined by the four ESIS factors along with student grade-level were regressed against the LTSR total score and the SUSSI total score in order to determine what classroom inquiry environmental factors potentially affected student science reasoning and NOS understanding. Prior to calculating regression equations however, missing data were imputed with the series mean using the SPSS $14^{\circledR}$ Replace Missing Values routine (SPSS, 2005). This analysis was completed separately for students of NBCTs and non-NBCTs and for middle school and high school teachers because when the teacher sample was disaggregated according to both simultaneously the cell-size was reduced to problematic levels.

Question 4: What is the impact of teacher perceptions factors impacting curriculum and limiting inquiry on the existence of inquiry learning environments? In addition to responding to instruments measuring NOS understanding (SUSSI) (Liang, et al., 2006) and the classroom inquiry behavior (ESIS) (Dunbar, 2002), teachers also responded to questions rating selected other factors potentially impacting their classroom inquiry practice (Table 3.14). Understanding the relationship among factors like these and classroom environment was important if changes in teacher experiences or institutional characteristics were to be suggested. In order to discern any patterns relating school-level instructional contexts to students' perceptions of classroom inquiry behaviors, ESIS teacher background question responses (Table 3.14) were correlated with the four ESIS factors emerging from student responses.

Teacher responses were disaggregated according to two different organizational schemes: Middle school or high school teacher, and NBCT or non-NBCT. Teacher background responses were structured using three different scales of variables and 
therefore required different methods of correlation. The Likert-type questions with five or six levels of response were correlated with the four ESIS factor sub-scores using Pearson product moment correlation. Those questions requiring ranking of the three most important factors impacting curriculum development or requiring responses of yes or no to effects impacting inquiry instruction were correlated with sub-scores using polyserial correlation. Polyserial correlation relates ordinal variables with continuous variables by assuming the ordinal variables have an underlying continuous construct.

Table 3.14

Teacher Background and Context Measures

Number graduate science courses

[values of 0 - none through 6 - more than five]

College science course scientific inquiry emphasis

[values of 0 - not at all through 5 - all courses very inquiry based]

Science education workshops in last five years

[values of 0 - none through 5 - more than four]

Conducted or assisted with scientific research

[values of 0 -never through 5 - more than once]

Familiarity with National Science Education Standards

[values of 0 - unaware through 5 - very familiar]

Familiarity with the Kentucky Program of Studies

[values of 0 - unaware through 5 - very familiar]

Familiarity with the Kentucky Core Content for Assessment

[values of 0 - unaware through 5 - very familiar]

Collaboration with other teachers planning science lessons

[0 - not at all through 5 - regular collaboration]

Rank of three most important factors in determining your science curriculum content

[values of 1,2, or 3]

District curriculum

State or national standards

Textbook

Available instructional materials

Guidance from principal

Other teachers' input

Other (specify)

Factors limiting use of the inquiry method of teaching science [yes or no]

There is nothing that limits the use of inquiry in my classroom

Lack of experience with inquiry, lack of awareness of inquiry

Reluctance to give up the role of primary source of classroom information

There isn't enough time 
Have tried it and it wasn't successful

Doubts about students' capability to do inquiry

Concern about discipline, large class size, potential chaos, etc.

Insufficient time and support for implementation

Insufficient background in science

Disagreement with the notion that inquiry is necessary

Doesn't have the necessary materials

Lack of parent and community support

Parental concerns about nonuse of science textbooks

Other (please specify in the space below)

\section{Classroom Level Variables}

Variables associated with classes selected for participation were collected (See Appendix F). These variables were those associated with general pedagogical practice identified from the National Education Longitudinal Study of 1988 (NELS:88) teacher survey instrument (NCES, 1988). Included were ratings of class demographic variables, use of instructional resources, ratings of classroom and professional climate, and standard professional behavior.

Analysis

Correlation. NBPTS certification has been demonstrated to identify effective teachers (Smith, Gordon, Colby \& Wang,, 2005; O’Sullivan, et al., 2005; Cavalluzzo, 2004; Vandevoort, Amrein-Beardsley \& Berliner, 2004; Goldhaber, \& Anthony, 2004; Bond, Smith, Baker \& Hattie, 2001). In order to provide some explanation for this correlation, teacher qualities should be studied and correlated with certification status. For example, teacher science inquiry instruction skills that were correlated with certification status may have actually been caused by teacher NOS understanding rather than pedagogical training or other factors. To discern the extent to which teacher science inquiry instructional strategies were related to teacher NOS understanding, teacher 
SUSSI scores (NOS understanding measure) were regressed on teacher ESIS scores (inquiry instructional practices).

\section{Validity}

\section{Construct Validity}

Issues of construct validity (Shadish, Cook, \& Campbell, 2002) enter this study in two ways. A debate continues in the science education community regarding the nature of science and pedagogical practices that support it (Abd-El-Khalick, Bell, \& Lederman, 1998; Abd-El-Khalick \& Lederman, 2000). Therefore any assertions of measurement of the nature of science are likely to be open to interpretation. In addition, questions may be raised about the extent to which the LTSR (Lawson, 2000) and SUSSI (Liang, et al., 2006) instruments measure important student science inquiry understandings and skills. Abd-El-Khalick and Lederman (2000) asserted that measuring participants' NOS understanding with an instrument consisting of prompts or questions created beforehand forces a false categorization of participants' philosophy into a researcher-defined classification that says as much about the researcher's perceptions and participants, and furthermore, that NOS conceptions should not be understood to be history-independent. This is to say that the most informed perspective as uncovered by an instrument created in the 1970s would not necessarily be the most informed perspective as exposed by a $21^{\text {st }}$ century instrument. Similarly, the extent to which the ESIS operationalizes the science inquiry pedagogy construct may be debated. These issues were ameliorated by comparison of results from the three instruments. 


\section{External Validity}

External validity describes the extent to which inferences made based upon this study's results may be generalized to the greater population of U.S. teachers and students. NBCT and non-NBCTs were purposively selected to represent the novice-expert dimension as well as to match the groups on school environment factors. For this reason, the extent to which inferences made from this study were generalizable to other populations of teachers depended on how well participating teachers modeled the population of teachers in Kentucky, or nationally.

All teachers selected for this study practiced in the state of Kentucky which has had a long-standing high-stakes reform environment (Kentucky Education Reform Act, 1990). Science inquiry has a central place in the Kentucky High School Core Content Document and is assessed in the Kentucky Core Content Test (KCCT). An argument can be constructed that this environment discourages focus on science inquiry instruction.

\section{Statistical Conclusion Validity}

Effect size. Cohen (1988) categorizes $\eta^{2}=0.02$ as a small effect size, $\eta^{2}=0.15$ as a moderate effect size, and $\eta^{2}=0.35$ as a large effect size. The effect sizes measured at various points during this study were all categorized as small, ranging from 0.01 to 0.06 . This may be because instruments used to measure science inquiry understanding in this study may have assessed constructs that were resistant to the inquiry instruction as operationalized in this study. For instance, Lawson's Test of Scientific Reasoning was initially designed to measure formal operational thinking (Lawson, 1978). As such, its score may have described intellectual development to a greater extent than science inquiry learning. 
In addition, Lederman and others have asserted that understanding of nature of science concepts is highly contextualized, causing students to understand questions to have very different meaning from the adults who constructed them (Lederman, Abl-ElKhalick \& Bell, 2002). For this reason, the pencil-and-paper instrument utilized to gather NOS data may have been insensitive to constructs being studied.

As was discussed earlier in this chapter, the period of instruction during which participating students were taught by their teachers represented only a small percentage of students' total educational experience. For this reason, the conceptual change students underwent may have been relatively small in size. A benefit for this study is that science instruction in elementary school is generally limited, as is science inquiry instruction at all levels. For these reasons differences science inquiry teaching among teacher groups may be detectable.

Sample size. The teacher sample for this study was small for creating statistically significant results. For certain analyses, too few subjects displaying pertinent professional or demographic factors were included in the study to enable statistically valid conclusions. Diversity of measurements including both quantitative and qualitative elements, however, was intended to ameliorate this weakness by generating other forms of evidence to explain emergent patterns or by describing phenomena that were not detectable using quantitative methods alone.

Self-report bias. When a subject is asked to reflect on his qualities or actions, there is a tendency to attempt to please the researcher by anticipating answers desired by the researcher or to attempt to bias responses to improve the subject's image (Cook \& Campbell, 1979). Teddlie and Stringfield (1993) found that teacher surveys did not 
accurately describe school climate, and that student survey was a much better measure. The researchers postulated that surprising result was because teacher perspective was confounded by instructional intentions and planning along with the distractions of teaching. Self-report bias was a validity threat in this study due to the prevalence of survey data in the analysis. Confirmatory student surveys, observation of randomly selected classrooms, and documentary analysis ameliorated the threat of self-report bias.. As in the work by Teddlie and Stringfield (1993), student responses in the present study were a much more sensitive measure of classroom climate than their teachers' responses. History. The students' science reasoning and understanding of NOS might have grown regardless of their experiences in science class. As this study design included no elements to track experiences of students in the months before the first phase of assessment, or between first and second phases of assessment, the possibility exists that other courses or experiences may have caused changes in their science inquiry knowledge. Furthermore, this study was designed to detect differences in teacher attributes and instructional decisions, but did not contain effective methods to trace the origin of those differences to particular experiences.

Maturation. The intellectual state of students in middle school and early high school is changing at a large rate. Students participating in this study might have shown improvement on the instruments regardless of what instruction was provided. This is especially true of the LTSR which has been shown to be sensitive to developmental level (Lawson, 1979). The pattern of results indicated that no significant changes occurred between phases of student science reasoning and NOS understanding assessment indicating that the threat of maturation to validity was not present. 


\section{Limitations}

Important answers to education questions should relate to solutions affecting student outcomes and, therefore, are causal in nature. This was a relatively small-sample, quasi-experimental study. Because random assignment to experimental and control conditions did not occur and effect sizes were small, the most general causal arguments are untenable. Therefore, results of this study should not be construed as providing definitive solutions for either identifying exemplary teachers or describing effective practices. However, this study was designed to distinguish among teachers based upon their NOS understanding, the classroom environment they create, and the consequent effect on student understanding while addressing important counterfactuals such as teacher science education, teaching experience, and pedagogical training. Consequently, the results of this study should be used to corroborate existing findings and to point toward fertile areas in which to design and conduct experimental studies.

\section{Inferences Regarding Teachers and Teacher Practice}

Although the teacher sample could not be randomly selected, teachers were purposively selected to be matched on important demographic factors. Even though all participants were drawn from the population of Kentucky middle and high school teachers because the sample represented the full spectrum of teachers and schools, results may be generalizable to the general population of U.S. science teachers. Because participating NBCTs were not studied before they attempted certification, conclusions should not be drawn regarding the effect of the certification process on teaching quality. Therefore this study can neither confirm nor refute other studies that have shown an effect on teacher quality due to attempting NBPTS certification; however, some 
inferences may be drawn from results correlating differences between teacher characteristics and specific teacher practices. Inferences from this study may suggest directions for research that are likely to provide important answers that improve teacher practice creating science inquiry learning environments.

This study included limited direct measures of teacher practice and did not attempt to richly describe student dispositional, achievement, or background variables. In-depth examination of the relationship between other pedagogical behaviors constituting the entire suite of effective science teacher behaviors and student achievement was not a purpose of this study. Instead, the study focused on the unique contribution of teacher characteristics to the science inquiry environments they create, and any subsequent effect these environments may have had on student science reasoning and NOS understanding. Within this scope, this study may begin to situate the creation of science inquiry learning environments within the corpus of effective middle and high school teacher practices that comprise the toolbox accomplished teachers bring to their regular instruction.

The only direct measures of teacher practice in this study were structured interviews of a stratified random sub-sample of participating teachers. The two observation protocols chosen to structure observations were well validated and shown to be reliable (Appeldoorn, 2004; Piburn \& Sawada, 2000). Observations occurred on a single occasion without calculation of inter-rater reliability, and did not include any systematic interviews. In spite of these limitations, the freedom participants were given to choose a lesson demonstrating their inquiry instruction and clear patterns evident in 
observation data lend strength to inferences that might be drawn from triangulating patterns observed in teacher and student surveys and assessments.

\section{Inferences Regarding the Effect of Teacher Characteristics and Practice on Student}

\section{Achievement}

This study was also designed to correlate teacher characteristics and classroom inquiry environment with student science reasoning and NOS understanding. However, measurement of these two student constructs relied on single instruments. This introduces a risk of mono-method bias into the design (Shadish, Cook, \& Campbell, 2002). Monomethod bias occurs because measurement methods are created using a particular definition of the construct being measured. Using a single measurement method, then, restricts inferences that may be drawn to those reflecting the single way in which the construct was operationalized by the instrument. Additionally, significant discussion exists in the science education field regarding whether nature of science may be assessed by fixed-response or Likert-type response instruments (Abd-El-Khalick \& Lederman, 2000). For this reason, caution should be taken when drawing inferences regarding either the nature or extent of nature of science understanding displayed by either teachers or students participating in this study. Trends in the data triangulating student achievement results with teacher characteristics or classroom inquiry environment descriptions may indicate what particular dimensions of student science reasoning or NOS understanding would provide direction for further studies.

The design of this study did not capture the entire arc of the instructional cycle from the beginning of the year through the end of the course, limiting inferences that can be drawn describing the effect of participating teachers on students' understanding. 
Because much of the variation in student achievement may have been related to other aspects of instruction not measured in this study, the study design was sensitive to only a small proportion of the total effect participating teachers may have had on student science reasoning or NOS understanding. Therefore, caution should be employed when attempting to draw broad inferences about science teacher quality or the effectiveness of science instructional strategies from these results. 


\section{RESULTS}

\section{Introduction}

Results discussed in this chapter are organized around the answers to the four research questions. Throughout the process, multiple analyses were completed on both teacher and student data. For this reason, multivariate tests would seem to be the most appropriate technique. Examination of the teacher sample however indicated that the sample size of 50 teachers decreased the cell-size to a few teachers for analysis, limiting the power of this method to detect differences. Nearly 1200 students participated in this study however, which did provide sufficient size for multivariate analysis.

Question I Findings:

National Board Certified science teachers' knowledge of the nature of science is significantly higher than their non-certified counterparts

\section{NOS Understanding Comparison}

In order to answer the research question about knowledge of science inquiry and nature of science for NBCTs compared to non-certified counterparts, independent-t tests were calculated for scores of each of the six SUSSI sub-categories and for the SUSSI total score. The SUSSI (Liang, 2006) consisted of six sets of four statements to which respondents were prompted to rate their agreement on a five-level Likert-type scale ranging from strongly disagree through strongly agree (see Appendix C). Responses were coded in decreasing order from strong agreement with the scientifically-informed 
statement $(+2)$ through unsure $(0)$ to strong agreement with the scientifically-uninformed statement $(-2)$. Scores were tabulated for each of the six sub-categories and for the total score. A sub-score of +8 would result from strong agreement with scientifically-informed views on all four questions comprising that sub-score, whereas a sub-score of -8 would indicate the strongest agreement with scientifically-uniformed views. Therefore, combining all six sub-scores, an individual who was perfectly informed regarding the nature of science would receive a total score of +48 on the SUSSI assessment. However, willingness to "strongly agree" $(+2)$ or "strongly disagree" $(-2)$ with a statement, as opposed to merely agreeing $(+1)$ or disagreeing $(-1)$, also may have been related to teacher personality traits in addition to teacher understanding of the concept reflected in the item. For this reason, agreement (not necessarily "strong agreement") with the most informed viewpoint (corresponding to a score of +1 ) was interpreted to indicate a scientifically solid understanding of inquiry and nature of science. When viewed from this perspective, a sub-score of +4 (and a total score of +24 ) indicated the teacher possessed an informed viewpoint on the nature of science. Sub-scores near zero with small standard deviation indicated a state of being unsure about the topic. Sub-total scores near zero with large standard deviations suggested agreement with both informed and uniformed views about the topic.

T-tests were used to compare NBCT and non-NBCT samples. Because the ratio of sample sizes between the non-NBCT sample and the NBCT sample was greater than $1.5 / 1$, the t-test was sensitive to non-normality in the data (Stevens, 2002). Levene's test for equality of variances was $0.144(\mathrm{df}=1,61, \mathrm{p}=0.705)$ indicating that no violation of the equality of variances assumption existed. The normality of the teacher SUSSI total 
score was calculated with the Shapiro-Wilk normality test statistic. For non-NBCT sample the statistic was $0.963(\mathrm{df}=42, \mathrm{p}=0.196)$, and for the NBCT sample the statistic was $0.920(\mathrm{df}=21, \mathrm{p}=0.086)$ indicating that neither distribution violated the normality assumption. For these reasons the significance of the t-statistic may be interpreted (Stevens, 2002). Because the two groups were being compared on seven variables however, a Bonferonni correction was appropriate (Stevens, 2002). The Bonferonni correction in this situation indicated that to be significant at the uncorrected $p<0.05$ level, significance in this case must be less than $\mathrm{p}=0.007$. Under this more conservative test, only three comparisons showed significance (see Table 4.1). Because one of the major purposes of this study is to locate relationships among teacher understanding, teacher practice, and student understanding that may lead to other productive studies however, uncorrected significance levels were reported as well.

NBCT status. Test descriptive statistics (mean and standard deviation) and the significance of the corresponding test statistics comparing groups of teachers SUSSI subscore and total scores are displayed in Table 4.1. As measured by the SUSSI instrument, NBCTs appeared to have significantly more informed views than non-NBCTs regarding methodology of scientific investigations and overall NOS understanding (see Table 4.1first section).

Table 4.1

Means and (Standard Deviations) of Teachers' SUSSI Scores across Five Dimensions of Teacher Categorization

\begin{tabular}{|c|c|c|c|c|c|c|c|}
\hline & $\begin{array}{l}\text { Observing } \\
\text { \& Inferring } \\
\text { in Science }\end{array}$ & $\begin{array}{c}\text { Change in } \\
\text { Theories }\end{array}$ & $\begin{array}{l}\text { Science } \\
\text { Laws vs. } \\
\text { Theories }\end{array}$ & $\begin{array}{c}\text { Social \& } \\
\text { Cultural } \\
\text { Influence }\end{array}$ & $\begin{array}{c}\text { Imagine } \\
\& \\
\text { Creativity }\end{array}$ & $\begin{array}{l}\text { Method } \\
\text { in } \\
\text { Science }\end{array}$ & $\begin{array}{l}\text { Total } \\
\text { Score }\end{array}$ \\
\hline \multicolumn{8}{|c|}{ NBPTS Certification ${ }^{a}$} \\
\hline non-NBCT & 0.94 & 2.06 & -2.35 & 2.61 & -1.32 & 1.32 & $\begin{array}{l}3.26 \\
857)\end{array}$ \\
\hline $\begin{array}{l}(\mathrm{n}=31) \\
\mathrm{NBCT}\end{array}$ & $\begin{array}{c}(1.81) \\
1.53\end{array}$ & $\begin{array}{c}(1.71) \\
2.32\end{array}$ & $\begin{array}{l}(2.73) \\
-1.79\end{array}$ & $\begin{array}{c}(2.78) \\
3.37\end{array}$ & $\begin{array}{c}(3.84) \\
0.74\end{array}$ & $\begin{array}{c}(2.95) \\
3.47\end{array}$ & $\begin{array}{c}(8.57) \\
9.63\end{array}$ \\
\hline
\end{tabular}




\begin{tabular}{|c|c|c|c|c|c|c|c|}
\hline$(\mathrm{n}=19)$ & $(1.84)$ & $(1.80)$ & $(3.26)$ & $(2.59)$ & $(4.48)$ & $(2.63)$ & $(9.90)$ \\
\hline p-value & 0.270 & 0.623 & 0.512 & 0.343 & $0.091^{\dagger}$ & $0.012 *$ & $0.020 *$ \\
\hline \multicolumn{8}{|c|}{ Middle/High School Assignment $^{a}$} \\
\hline Middle & 1.00 & 2.14 & -3.27 & 2.82 & -2.68 & 2.09 & 2.09 \\
\hline$(\mathrm{n}=28)$ & $(1.83)$ & $(1.21)$ & $(2.37)$ & $(2.34)$ & $(3.21)$ & $(2.69)$ & $(7.11)$ \\
\hline High & 1.29 & 2.18 & -1.25 & 2.96 & 1.14 & 2.18 & 8.50 \\
\hline$(n=22)$ & $(1.84)$ & $(2.08)$ & $(3.04)$ & $(3.00)$ & $(4.12)$ & $(3.27)$ & $(10.32)$ \\
\hline $\mathrm{p}$-value & 0.587 & 0.933 & $0.013^{*}$ & 0.852 & $0.001 * * *$ & 0.919 & $0.017 *$ \\
\hline \multicolumn{8}{|c|}{ Non-Education Degree } \\
\hline None & 1.71 & 1.67 & -3.29 & 2.14 & -3.00 & 2.29 & 2.22 \\
\hline$(\mathrm{n}=7)$ & $(2.14)$ & (1.13) & $(3.20)$ & $(2.12)$ & $(3.70)$ & $(1.70)$ & $(7.78)$ \\
\hline BA/BS & 1.19 & 2.08 & -2.24 & 2.92 & -0.24 & 2.32 & 6.03 \\
\hline$(n=37)$ & $(1.75)$ & $(1.89)$ & $(2.83)$ & $(2.79)$ & $(4.37)$ & $(2.89)$ & $(9.13)$ \\
\hline $\mathrm{MS} / \mathrm{PhD}$ & 0.33 & 2.33 & -0.17 & 3.67 & 0.50 & 0.50 & 7.50 \\
\hline$(n=6)$ & (1.97) & $(1.37)$ & $(2.64)$ & (2.94) & (2.59) & (2.59) & (13.94) \\
\hline p-value & 0.389 & 0.646 & 0.195 & 0.539 & 0.383 & 0.444 & 0.473 \\
\hline \multicolumn{8}{|c|}{ Certification Rank } \\
\hline Rank III & 1.57 & 0.257 & -2.43 & 3.00 & -2.86 & 0.57 & 2.43 \\
\hline$(n=7)$ & $(0.54)$ & $(1.13)$ & $(0.98)$ & $(2.24)$ & $(4.30)$ & $(2.30)$ & $(6.85)$ \\
\hline Rank II & 0.82 & 1.76 & -2.76 & 2.41 & -1.53 & 1.29 & 2.00 \\
\hline$(n=17)$ & $(2.04)$ & $(2.02)$ & $(2.86)$ & $(3.36)$ & $(3.52)$ & $(3.29)$ & $(9.08)$ \\
\hline Rank I & 1.27 & 2.31 & -1.65 & 3.19 & 0.73 & 3.12 & 8.96 \\
\hline$(n=26)$ & $(1.91)$ & $(1.67)$ & $(2.27)$ & $(2.38)$ & $(4.24)$ & (2.69) & $(9.50)$ \\
\hline $\mathrm{p}$-value & 0.607 & 0.488 & 0.466 & 0.656 & $0.060^{\dagger}$ & $0.046 *$ & $0.036 *$ \\
\hline \multicolumn{8}{|c|}{ NBCT-School Level Interaction ${ }^{b}$} \\
\hline NM & 0.73 & 2.27 & -3.20 & 3.07 & -2.33 & 1.40 & 1.93 \\
\hline$(n=16)$ & (1.63) & $(1.28)$ & $(2.04)$ & $(2.55)$ & $(3.24)$ & $(2.92)$ & (7.29) \\
\hline $\mathrm{NH}$ & 1.13 & 1.88 & -1.56 & 2.19 & -0.38 & 1.25 & 4.50 \\
\hline$(n=15)$ & $(1.10)$ & $(2.06)$ & $(3.10)$ & $(2.99)$ & $(4.21)$ & $(3.07)$ & $(9.68)$ \\
\hline YM & 1.57 & 1.86 & -3.43 & 2.29 & -3.43 & 3.57 & 2.43 \\
\hline$(n=12)$ & $(2.23)$ & (1.07) & (3.16) & $(1.89)$ & $(3.26)$ & $(1.27)$ & $(7.25)$ \\
\hline YH & 1.50 & 2.58 & -0.83 & 4.00 & 3.17 & 3.42 & 13.83 \\
\hline$(n=7)$ & (1.68) & $(2.11)$ & (3.04) & $(2.80)$ & $(3.10)$ & $(3.23)$ & $(8.90)$ \\
\hline p-value & 0.672 & 0.712 & $0.091^{\dagger}$ & 0.326 & $0.000 * * *$ & 0.103 & $0.004^{* *}$ \\
\hline
\end{tabular}

Note. The p-value rows indicate significance of the score differences between groups represented in each table section.

a Results of independent t-tests.

${ }^{b}$ Results of ANOVA.

${ }^{\dagger} \mathrm{p}<0.10 . * \mathrm{p}<0.05 . * * \mathrm{p}<0.01 . * * * \mathrm{p}<0.001$

Two trends should be noted here as they will become important in light of further analysis. Two sub-scores were notably smaller than others: Scientific Laws vs. Theories, 
and Imagination and Creativity in Scientific Investigations. This trend existed throughout the teacher sample, regardless of experience or training, and throughout the student sample regardless of grade-level. This trend will be addressed in the discussion chapter.

Middle/high school status. Because the pre-service educational experiences, course assignment, and school climates of middle and elementary school teachers are markedly different from those of high school teachers, teachers were also compared across middle/high school assignment (Table 4.1-second section). Data analysis reveal that high school teachers were significantly better informed regarding the difference between scientific laws and theories $(\mathrm{p}<0.05)$, the role of imagination and creativity in science $(p<0.001)$, and overall NOS understanding $(p<0.05)$.

Non-education degree. The third section of Table 4.1 displays SUSSI score categorized by non-education degree level. The analysis of variance (ANOVA) of teacher SUSSI score with teacher non-education degree level indicated that there was no significant difference in teacher NOS understanding between three levels of noneducation degree level (No degree, $\mathrm{BA} / \mathrm{BS}, \mathrm{MA} / \mathrm{MS} / \mathrm{PhD}$ ). However, examination of the differences in total scores (2.22 for no degree, 6.03 for Bachelors, 7.50 for Masters) suggested there may be a difference that this sample did not adequately capture. Small sample size or widely unequal sample size among groups may have attenuated the statistical significance comparing these groups (Table 4.1-third section). Because nearly all scores of teachers without any non-education degree were lower than those of teachers with other degrees (typically science-related degrees), there may be reason to suspect that trends existed in teacher responses that the ANOVA was unable to detect. 
Certification rank. The fourth section of Table 4.1 displays data describing teacher NOS understanding by teacher certification rank. Examination of possible differences among these three groups was important because increasing certification rank corresponds to increased pedagogical training. Analysis of variance among these three groups indicated that significant differences existed between teacher ranks for the subscores of Imagination in Science, Methodology in Science, and Total Score (Table 4.1fourth section). Tukey post hoc analysis indicated that the difference in Total Score originated from differences between Ranks I and II. Rank I and Rank III did not differ significantly, but this may be because Rank III group size was too small to enable estimation of significance (Stevens, 2002). This lack of significant difference also may have been an artifact of the cell-size differences between NBCTs and non-NBCTs. NBCT status-middle/high interaction. Differences in education and training were detected between middle school and high school teachers. In order to explore the interaction between NBCT status and middle/high school-level assignment, NBCT status and middle/high school teaching assignment were combined into a four-level variable against which SUSSI sub-scores and total score were compared. This was necessary because both NBCT status and middle/high school assignment had only two levels, and therefore, ANOVA post hoc tests were not possible for either. The last section of Table 4.1 displays numbers of teachers represented by each level of the NBCT-School-Level interaction variable. This variable was labeled using a two-character system in which the first letter corresponds to NBCT status $(\mathrm{Y} / \mathrm{N})$ and the second letter corresponds to middle/high school assignment (M/H). Significant differences existed for Imagination in Science and Total Score measures. Tukey post hoc analyses indicated that this 
significance occurred because high school NBCTs scored significantly higher than middle school NBCTs and either high school or middle school non-NBCTs.

\section{Summary}

Significant results. Middle and high school science NBCTs outperformed their non-NBCT counterparts on the omnibus SUSSI Total Score and on the Science Methodology sub-scale at the $\mathrm{p}<0.05$ level and on the Imagination and Creativity in Science sub-scale at the $\mathrm{p}<0.10$ level. High school teachers outperformed middle school teachers at the $\mathrm{p}<0.05$ level on the Science Laws vs. Theories sub-scale, but this only reflected high school teachers' state of being less uninformed—meaning both high school and middle school teachers' scores reflected levels of uninformed understanding. Additionally, high school teachers outperformed middle school teachers at the $\mathrm{p}<0.001$ level on the Imagination and Creativity in Science sub-scale and at the $\mathrm{p}<0.05$ level on the Total Score.

Significant differences were detected between teachers grouped by certification rank on the Imagination and Creativity in Science and Method in Science sub-scores and on Total Score. In this comparison, increasing rank benefited teachers on both sub-scales, and Rank I status benefited teachers on the Total Score. In this case, Rank I status may have served as a proxy for NBCT status. When teachers were grouped by the four-level NBCT — middle/high school assignment variable, high school NBCTs outperformed all other teachers at the $\mathrm{p}<0.10$ level on the Science Laws vs. Theories subscale, at the $\mathrm{p}<$ 0.001 level on the Imagination and Creativity in Science sub-scale, and at the $\mathrm{p}<0.01$ level on the Total Score. 
Non-significant results. No differences in teacher NOS understanding were found between the three levels of non-education degree (none, $\mathrm{BA} / \mathrm{BS}$, or MA/MS/PhD). This is interesting as it tends to validate previous research indicating implicit NOS instruction is less effective changing levels of understanding (Abd-El-Khalick \& Lederman, 2000). Also, no differences were detected for any group of teachers on the Observing and Inferring in Science, Changes in Theories, or Social and Cultural Influence in Science sub-scores.

Overall. Significant differences on understanding nature of science were detected between middle and high school teachers and between NBCTs and their non-NBCT counterparts. NBCTs have more informed views of NOS as do high school teachers. Furthermore, differences seem to be most evident in the Imagination and Creativity in Science and Method in Science sub-scales, but differences on all sub-scales tended to benefit NBCTs and high school teachers contributing to significantly higher overall NOS understanding as well. Interestingly, teachers grouped by non-education degree was the single comparison that did not display any significance.

\section{Question 2 Findings:}

NBCTs create classroom learning environments in which their students display science inquiry behaviors more frequently than those created by their non-certified counterparts

This study relied on two sources of data of classroom inquiry behaviors: A survey of perceived instructional behaviors and observation of a sample of teachers. Use of the survey enabled the sampling of a larger proportion of classroom inquiry environments, but it also introduced self-report bias as a potential counterfactual. Observation of a 
representative sample of teachers provided data to triangulate with the teacher survey, enhancing the trustworthiness of interpretations.

\section{Classroom Inquiry Environment Description}

The ESIS survey (Dunbar, 2002) was administered to both middle and high school science teachers and their students in order to obtain data addressing potential teacher self-report bias. Each survey item was coded so that higher values corresponded to more frequent science inquiry practice. Responses to each item were rated on a scale from 0 to 5 . Thus the total score for the 29 classroom inquiry items on the instrument scaled between 0 and 145 points. Higher values indicated more frequent classroom behaviors supportive of student science inquiry and less frequent behaviors that inhibit it. Because a variety of science instructional strategies have been shown to be effective, very high scores on this scale did not necessarily indicate better instruction. Instead, the optimal frequency of classroom inquiry practice should be established by correlating the score on this instrument it with maximal student achievement. Table 4.2 summarizes the behaviors assessed by each ESIS item. Table 4.3 displays teacher and student means and standard deviations for responses to each item.

Table 4.2

ESIS Teacher Behavior Variable Assignment

\begin{tabular}{|c|c|}
\hline Item & Teacher Behavior \\
\hline & [values of $0-$ never through $5-$ exclusively] \\
\hline $\mathrm{E}^{\mathrm{a}}$ & Memorize scientific facts or information independently? \\
\hline E2 & Use data to construct a reasonable explanation? \\
\hline E3 & Seek and recognize patterns (trends in the data or observations)? \\
\hline E4 & Detect sequences (the order of events in a science activity)? \\
\hline $\mathrm{E}^{\mathrm{a}}$ & Follow a set series of steps to get the right answer to a question? \\
\hline & [values of $0-$ never through $5-5 x$ or more per week] \\
\hline E6 & $\begin{array}{l}\text { Ask questions during investigations that lead to further ideas, questions and } \\
\text { investigations? }\end{array}$ \\
\hline$E 7^{\mathrm{a}}$ & $\begin{array}{l}\text { Wait to act until the teacher gives instructions for the next step in the } \\
\text { investigation? }\end{array}$ \\
\hline
\end{tabular}




\begin{tabular}{ll}
\hline E8 & $\begin{array}{l}\text { Use science process skills (e.g., observing, classifying, recording data) } \\
\text { connected to a specific science topic? }\end{array}$ \\
E9 & $\begin{array}{l}\text { Choose appropriate tools for an investigation? } \\
\text { E10 }\end{array}$ \\
Wait for the teacher's explanation before expressing an observation or \\
conclusion?
\end{tabular}

${ }^{a}$ Negatively worded item reverse coded in the analysis.

Table 4.3

ESIS Results for non-NBCT and NBCT Groups

\begin{tabular}{lcccc}
\hline & \multicolumn{2}{c}{ non-NBCT } & \multicolumn{2}{c}{ NBCT } \\
Teacher \\
Item & Mean (SD) & $\begin{array}{c}\text { Student } \\
(\mathrm{n}=647 \text { to 669) } \\
\text { Mean (SD) }\end{array}$ & $\begin{array}{c}\text { Teacher } \\
(\mathrm{n}=19)\end{array}$ & $\begin{array}{c}\text { Student } \\
(\mathrm{n}=394 \text { to 406) } \\
\text { Mean (SD) }\end{array}$ \\
\hline E1 $^{\text {a }}$ & $3.1(0.788)$ & $2.0(1.003)$ & $3.0(0.745)$ & $2.0(0.924)$ \\
E2 & $2.5(0.626)$ & $2.3(0.925)$ & $2.6(0.607)$ & $2.4(0.880)$ \\
E3 & $2.4(0.615)$ & $2.3(1.598)$ & $2.7(0.562)$ & $2.3(0.900)$ \\
E4 & $2.3(0.682)$ & $2.1(1.030)$ & $2.2(0.647)$ & $2.2(0.958)$ \\
E5 $^{\text {a }}$ & $2.5(0.677)$ & $2.5(1.073)$ & $2.5(0.618)$ & $2.7(0.949)$ \\
E6 $^{\text {a }}$ & $2.2(1.424)$ & $2.2(1.772)$ & $2.1(1.580)$ & $2.7(1.531)$ \\
E7 $^{\text {a }}$ & $3.2(1.584)$ & $2.6(1.591)$ & $3.1(1.026)$ & $2.7(1.479)$
\end{tabular}




\begin{tabular}{lcccc} 
E8 & $2.4(1.358)$ & $2.3(1.464)$ & $2.6(1.170)$ & $2.6(2.114)$ \\
E9 & $1.5(1.091)$ & $2.4(1.513)$ & $1.8(1.032)$ & $2.5(1.507)$ \\
E10 $^{\text {a }}$ & $3.6(1.331)$ & $2.6(1.630)$ & $3.4(1.300)$ & $2.6(1.522)$ \\
E11 & $2.3(1.222)$ & $2.4(1.490)$ & $2.6(1.216)$ & $2.6(1.404)$ \\
E12 & $2.5(1.313)$ & $2.4(2.349)$ & $3.3(1.447)$ & $2.7(2.587)$ \\
E13 & $2.0(1.291)$ & $2.4(1.682)$ & $1.9(1.286)$ & $2.6(1.388)$ \\
E14 & $1.1(1.118)$ & $2.2(1.770)$ & $0.8(0.713)$ & $2.3(1.457)$ \\
E15 & $1.6(1.112)$ & $2.7(1.614)$ & $1.9(1.268)$ & $2.8(1.495)$ \\
E16 & $1.9(1.237)$ & $2.0(2.223)$ & $1.9(1.129)$ & $2.0(1.324)$ \\
E17 & $3.4(1.547)$ & $1.9(1.463)$ & $4.1(1.663)$ & $1.9(1.494)$ \\
E18 & $1.5(1.121)$ & $2.3(2.041)$ & $1.5(0.964)$ & $2.4(1.520)$ \\
E19 & $1.6(1.199)$ & $2.1(2.358)$ & $1.9(1.487)$ & $2.1(1.640)$ \\
E20 ${ }^{\text {a }}$ & $3.5(1.092)$ & $2.4(1.694)$ & $3.8(1.425)$ & $2.1(1.641)$ \\
E21 & $2.5(1.411)$ & $2.5(1.669)$ & $3.0(1.414)$ & $2.5(1.502)$ \\
E22 & $2.1(1.544)$ & $2.3(1.55)$ & $2.2(1.537)$ & $2.5(1.461)$ \\
E23 & $2.0(1.155)$ & $2.5(1.534)$ & $2.3(1.447)$ & $2.7(1.532)$ \\
E24 ${ }^{\text {a }}$ & $2.9(1.231)$ & $2.5(1.467)$ & $2.8(1.686)$ & $2.8(1.531)$ \\
E25 & $3.8(1.440)$ & $2.9(1.570)$ & $4.1(0.809)$ & $3.2(1.650)$ \\
E26 & $4.0(1.169)$ & $2.6(1.969)$ & $4.1(0.970)$ & $2.9(1.578)$ \\
E27 & $3.0(1.509)$ & $2.5(1.607)$ & $3.6(1.165)$ & $2.7(1.522)$ \\
E28 ${ }^{\text {a }}$ & $3.9(1.204)$ & $2.4(1.500)$ & $4.1(0.737)$ & $2.4(1.440)$ \\
E29 & $2.4(1.427)$ & $2.6(1.756)$ & $2.4(1.610)$ & $2.7(1.567)$ \\
\hline Total & $61.32(14.732)$ & $72.01(15.346)$ & $64.37(13.889)$ & $72.79(14.259)$ \\
\hline a This
\end{tabular}

${ }^{\mathrm{a}}$ This item is negatively coded. In all cases higher values indicate higher levels of inquiry instruction.

Analysis of Classroom Science Inquiry Behaviors

Student responses to the ESIS survey appeared to be more sensitive to classroom climate than those of their teachers. For this reason, aggregate student ESIS responses rather than teacher responses were used to characterize each classroom. Additionally, because differences in teacher NOS understanding between middle and high school teachers were detected, analysis of classroom inquiry behaviors was completed separately for middle and high school teachers. As is described in the methodology chapter, a factor analysis of student ESIS responses resulted in five factors, one of which was represented 
only a single negatively coded response. For this reason, only the first four ESIS factors were included in the analysis.

Student responses were disaggregated according to middle or high school enrollment. Multivariate analysis of variance (MANOVA) was completed using NBCT status, teacher experience level, and non-education degree as independent variables and student responses to the first four ESIS factors as dependent variables. At the high school level, students of NBCTs rated their teacher significantly higher on all classroom inquiry environment factors than did those of non-NBCTs, and no other independent variable contributed significantly (Table 4.4). At the middle school level, a significant difference in classroom inquiry environment was associated only with interactions NBCT status with teacher variables-teacher experience and non-education degree (Table 4.5). MANOVA indicated that for middle school students the interaction between NBCT status and teacher experience level, along with the interaction between NBCT status and non-education degree level contributed significantly to their perceptions of classroom inquiry environment.

Table 4.4

Multivariate and Univariate Analyses of Variance F Ratios for High School NBCT, NonEducation Degree, and Experience Level, on Inquiry Environment Factors

\begin{tabular}{|c|c|c|c|c|c|}
\hline \multirow[b]{2}{*}{ Variable } & \multirow[b]{2}{*}{ MANOVA } & \multicolumn{4}{|c|}{ ANOVA } \\
\hline & & Investigation & $\begin{array}{l}\text { Use of Data } \\
\text { to Explain }\end{array}$ & $\begin{array}{c}\text { Science } \\
\text { Dialogue }\end{array}$ & $\begin{array}{l}\text { Approach } \\
\text { to Science }\end{array}$ \\
\hline $\operatorname{NBCT}(\mathrm{N})$ & $4.860^{\mathrm{a} * * *}$ & $16.274^{\mathrm{c}} * * *$ & $4.096^{\mathrm{c}} *$ & $14.041^{\mathrm{c} * * *}$ & $4.793^{\mathrm{c} *}$ \\
\hline Degree (D) & $0.162^{\mathrm{a}}$ & $0.113^{\mathrm{c}}$ & $0.149^{\mathrm{c}}$ & $0.091^{\mathrm{c}}$ & $0.001^{\mathrm{c}}$ \\
\hline Experience (E) & $0.835^{\mathrm{b}}$ & $2.416^{\mathrm{d} \dagger}$ & $0.339^{\mathrm{d}}$ & $0.482^{\mathrm{d}}$ & $1.241^{\mathrm{d}}$ \\
\hline$N \times D$ & $3.264^{\mathrm{a} *}$ & $11.513^{\mathrm{c} * * *}$ & $2.580^{\mathrm{c}}$ & $9.705^{\mathrm{c} * *}$ & $6.791^{\mathrm{c}_{* *}}$ \\
\hline $\mathrm{N} \times \mathrm{E}$ & $4.688^{\mathrm{a} * *}$ & $8.242^{\mathrm{C} * * *}$ & $5.500^{\mathfrak{c}} *$ & $10.038^{\mathfrak{C}} * *$ & $11.721^{\mathrm{c} * * *}$ \\
\hline $\mathrm{D} \times \mathrm{E}$ & $1.345^{\mathrm{b}}$ & $2.764^{\mathrm{d} \bar{t}}$ & $0.737^{\mathrm{d}}$ & $2.287^{\mathrm{d}}$ & $1.544^{\mathrm{d}}$ \\
\hline
\end{tabular}

Note. F ratios are Wilk's approximations of Fs.

${ }^{\mathrm{a}}$ Multivariate $\mathrm{df}=4,479 .{ }^{\mathrm{b}}$ Multivariate $\mathrm{df}=8,958 .{ }^{\mathrm{c}}$ Univariate $\mathrm{df}=1,482$.

${ }^{\mathrm{d}}$ Univariate $\mathrm{df}=2,482$.

${ }^{\dagger} \mathrm{p}<0.01$. * $\mathrm{p}<0.05$. ** $\mathrm{p}<0.01$. *** $\mathrm{p}<0.001$. 
Table 4.5

Multivariate and Univariate Analyses of Variance F Ratios for Middle School NBCT, Non-Education Degree, and Experience, on Inquiry Environment Factors

\begin{tabular}{lccccc}
\hline & & \multicolumn{4}{c}{ ANOVA } \\
\cline { 3 - 6 } \multicolumn{1}{c}{ Variable } & MANOVA & Investigation & $\begin{array}{c}\text { Use of Data } \\
\text { to Explain }\end{array}$ & $\begin{array}{c}\text { Science } \\
\text { Dialogue }\end{array}$ & $\begin{array}{c}\text { Approach to } \\
\text { Science }\end{array}$ \\
\hline NBCT (N) & $1.224^{\mathrm{a}}$ & $3.568^{\mathrm{c}} \mathrm{c}$ & $0.440^{\mathrm{c}}$ & $0.217^{\mathrm{c}}$ & $1.179^{\mathrm{c}}$ \\
Degree (D) & $1.813^{\mathrm{b} \dagger}$ & $2.254^{\mathrm{d}}$ & $0.177^{\mathrm{d}}$ & $1.885^{\mathrm{d}}$ & $0.070^{\mathrm{d}}$ \\
Experience (E) & $1.656^{\mathrm{b}}$ & $2.841^{\mathrm{d} \dagger}$ & $0.665^{\mathrm{d}}$ & $1.613^{\mathrm{d}}$ & $0.487^{\mathrm{d}}$ \\
N x D & $2.573^{\mathrm{a} *}$ & $2.024^{\mathrm{c}}$ & $1.389^{\mathrm{c}}$ & $0.301^{\mathrm{c}}$ & $4.108^{\mathrm{d}^{\mathrm{c}} *}$ \\
N x E & $2.894^{\mathrm{a} *}$ & $4.819^{\mathrm{c} *}$ & $7.111^{\mathrm{c} * *}$ & $8.195^{\mathrm{c} * *}$ & $7.854^{\mathrm{c} * *}$ \\
D x E & $1.075^{\mathrm{b}}$ & $1.550^{\mathrm{d}}$ & $0.101^{\mathrm{d}}$ & $2.675^{\mathrm{d}}$ & $0.873^{\mathrm{d}}$ \\
\hline
\end{tabular}

Note. F ratios are Wilk's approximations of Fs.

${ }^{\mathrm{a}}$ Multivariate $\mathrm{df}=4,353 .{ }^{\mathrm{b}}$ Multivariate $\mathrm{df}=8,706 .{ }^{\mathrm{c}}$ Univariate $\mathrm{df}=1,356$.

${ }^{\mathrm{d}}$ Univariate $\mathrm{df}=2,356$.

${ }^{\dagger} \mathrm{p}<0.01,{ }^{*} \mathrm{p}<0.05, * * \mathrm{p}<0.01$

\section{Other Classroom Pedagogical Practices}

Data was also collected from participating teachers regarding their general pedagogical practice (Table 4.6). Of these variables, independent-t tests between NBCT and non-NBCT groups indicated the only significant response was use of audio-visual materials $\mathrm{t}(46)=2.134, \mathrm{p}=0.038$. MANOVA was used to compare responses to these questions from teachers across experience level and non-education degree. Only Use of other Materials was significant at the $\mathrm{p}<0.10$ level in a comparison among the three levels of teacher experience $F(2,46)=2.617, p=0.089$. In general, these results showed that other general pedagogical practices beyond use of inquiry were similar for teachers independent of their NBCT status, years' experience, or non-education degree status.

Table 4.6

Teacher General Pedagogical Practice Variables

\section{Description}

1. Amount of homework assigned each week [number of hours/week]

2. Frequency of homework interactions [0-never thru Always-3]

- Keep records of who turned in assignment

- Return assignments with grades or corrections

- Discuss assignments in class 
3. Use of instructional materials [Not used-0 thru Primarily-3]

- Textbooks

- Reading materials other than textbooks

- Audio-visual materials

- Other

4. Percentage of textbook covered in course $[0-49 \%-0 ; 50-59 \%-1 ; 60-69 \%-2 ; 70-79 \%-3 ; 80-89 \%-4 ; 90-99 \% \& 100 \%-5]$

5. Opinion regarding course textbook [SD- $-2 ; \mathrm{D}--1 ; \mathrm{N}-0 ; \mathrm{A}-+1 ; \mathrm{SA}-+2$ ]

- Reading level is too difficult

- Helps develop problem-solving skills

- Explains concepts clearly

- Good suggestions for homework assignments

- Good job covering subject matter

- Considered interesting by most students

6. Time spent in class each week on various activities [0- None through 5-5+hours]

- Providing whole-class instruction

- Providing small-group instruction

- Providing individual-student instruction

- Maintaining order/discipline

- Administering tests

- Performing routine administrative tasks

- Conducting labs

\section{Classroom Observation}

In order to triangulate data, stratified random sampling was used to select eight teachers from participants who agreed to be observed. Attempts were made to distribute these observations across NBCT status and middle school or high school assignment. Table 4.7 displays NBCT-status, grade-level and content area taught, and number of years' experience of each observed teacher. Teachers are identified by pseudonym.

Table 4.7

Characteristics of Observed Teachers

\begin{tabular}{lcrlc}
\hline Pseudonym & NBCT status & Grade & Content & Experience \\
\hline Susan & $\mathrm{Y}$ & $11^{\text {th }}$ & Physical Science & 29 \\
Audrey & $\mathrm{Y}$ & $11^{\text {th }}$ & Chemistry & 28 \\
Robert & $\mathrm{N}$ & $10^{\text {th }}$ & Intro to Chemistry and Physics & 9 \\
Ted & $\mathrm{Y}$ & $7^{\text {th }}$ & $7^{\text {th }}$ Grade Science & 15 \\
Rhonda & $\mathrm{N}$ & $7^{\text {th }}$ & $7^{\text {th }}$ Grade Science & 2 \\
Jackie & $\mathrm{N}$ & $7^{\text {th }}$ & $7^{\text {th }}$ Grade Science & 4 \\
Kyle & $\mathrm{N}$ & $8^{\text {th }}$ & $8^{\text {th }}$ Grade Science & 32 \\
Jennie & $\mathrm{N}$ & $6^{\text {th }}$ & $6^{\text {th }}$ Grade Science & 6 \\
\hline
\end{tabular}


Teachers were observed on a date convenient for them on which they indicated they would be teaching using an inquiry-oriented pedagogy. Two observation protocols were used: The Reformed Teaching Observation Protocol (RTOP) (Piburn \& Sawada, 2000), and the CETP Classroom Observation Protocol (Lawrenz, Huffman, \& Appleldoorn, 2002). The RTOP is a high-inference protocol evaluating the nature of teacher and student discourse. It includes subscales rating: Lesson design and implementation, content, procedural knowledge, and classroom culture. It generates both a total score and five sub-scores. The CETP is a low-inference protocol that tallies teacher and student behaviors during five-minute intervals during the course of instruction. It rates instructional strategy, student engagement, and cognitive activity.

Each observation occurred on a date of the teacher's choosing on which he or she was teaching using science inquiry related instruction. With the exception of Audrey who taught on a $4 \times 4$ block schedule, all observations occurred late in the semester in the class participating in the study. Most observations occurred in January which, in Audrey's case, meant that she was teaching a new class in a new semester. This did not present a problem for the study because the observation was intended to triangulate teacher behaviors and not student behaviors. What follows is a short description of each teacher's science instruction and the learning environment he or she created.

Susan

Teacher and school description. Susan is an experienced National Board Certified chemistry teacher. She has a master's degree in animal science. That she attained National Board Certification in 2000 when there were few NBCTs in Kentucky is evidence of her professionalism and innovative character. Susan clearly feels comfortable 
in her position both in her classroom and her school. She claimed simultaneously to have a strong relationship with the principal, at the same time she believed the assistant principals were frustrated with her because she tended to interpret the school rules in favor of keeping students in her class instead of sending them off for behavioral consequences. Her master's degree in animal science originated from in her interest in horses. When she retires in a couple years, Susan may open a stable.

The school where Susan teaches is the only high school in her small rural county. Approximately 28 teachers teach the 430 students who are enrolled. The school is about 95\% white with fewer than 5\% African American or Hispanic students. The school is not recognized either as a Title I school, or a magnet school, and no free or reduced-price lunch data are available (NCES, 2007). Susan's school uses an A-B block schedule which effectively doubles the length of each class period, increasing the length of the school day so that it encompasses to two calendar days instead of one. This means students attend each class in their schedule every other day for the entire year.

Observation description and analysis. The course observed was $11^{\text {th }}$ grade physical science. The science class of 25 students represented a mix of student abilities and interests. The length of the class was 90 minutes. The entire period was filled with student investigation with very little teacher-directed instruction. The lesson was a continuation of an investigation using titration to determine the citric acid content of food. Class began with "What's your favorite indicator?" an activity Susan used to engage the class as she took attendance. She only allowed students to choose phenolphthalein as their favorite indicator if they could spell it. 
After this, for the first 20 minutes she used choral questioning to review the previous day's activity and set the stage for the day's learning experience. Responses to Susan's questions were politely provided, and no segment students monopolized the discussion or refrained from participating. Students had brought food samples to analyze for citric acid content. Susan provided equipment and indicator, but students were to create the procedure and control variables. She explicitly scaffolded instruction regarding controlling variables in her decision to not calibrate the indicator and in the discussion she coordinated regarding the consequences of that decision for her students' procedures.

The effect scaffolding instruction had on her students experience was distinct. All students remained engaged for the entire class session. Student-student and studentteacher dialogue was multi-faceted. Students volunteered to the teacher that they were energized by responsibility to design their own procedure. The student work-product of this lesson was a lab report intended to be a portfolio entry. Students finished the class period writing this portfolio entry.

Document analysis. Susan created the documents supporting this inquiry activity (see Appendix G1). Included was a document from the previous day's lesson that contained a general discussion section describing ascorbic acid as an example of an acid, along with its biological role in human physiology. This activity prepared the students to titrate food product to determine ascorbic acid content. The questions associated with this activity included those assessing basic declarative knowledge about ascorbic acid, procedural knowledge describing the experiment, and higher order understanding justifying inferences from observation. 
The document associated with the current day's activity was a rubric describing the writing portfolio piece, the work-product associated with the students' activity. The portfolio writing was a technical lab report describing the student-created procedure testing the ascorbic acid content of food products the students brought to class. Susan's rubric included a list of required elements accompanied by descriptions and guiding questions. The rubric provided limited feedback for students during the writing process, therefore, they would require either extensive knowledge of standard lab report formats, or interaction with a science teacher during the revision period.

Audrey

Teacher and school description. Audrey is an experienced National Board Certified chemistry teacher. She was one of the first two science teachers certified in Kentucky by the NBPTS in 1999. She also expresses confidence in her role in her school. Audrey discussed how, during discussions regarding schedule changes at her school, she told the administration they could make any changes they wanted but they couldn't change her block schedule. She described this exchange in a way that made clear her expectation was that her request would be honored.

Like Susan, Audrey's school is the only high school in her county. Approximately 80 teachers teach about 1500 students. The student body is approximately $95 \%$ white, $3 \%$ African American, with the remaining 2\% split between Hispanic, Native American, and Asian American. Audrey's school is not identified as a magnet school or a Title I school, and no free or reduced-price lunch data are available (NCES, 2007). The school utilizes a $4 \times 4$ block schedule so the entire arc of instruction for a course occurs in one semester. This meant that because Audrey was observed in January, she was teaching a class that 
did not participate in the assessment. Additionally and, more importantly, the observed class was at the beginning of the arc of instruction rather than at its end as was the case for the other teachers.

Observation description and analysis. On arrival, Audrey's students understood they were to situate themselves for class. Immediately upon arrival students wrote answers to pre-lab questions on a half-sheet of paper as a check on out-of-class work. During this time Audrey consulted a student regarding her sub-standard performance regarding the lab safety rules quiz which was preventing the student from participating in any laboratory activity. When finished, students passed their work forward following a predetermined routine. Audrey then discussed the answers to the pre-lab questions with the class. Within this discussion was an exchange focusing on the role of logic, evidence, imagination, and creativity in science, and the impact of bias in science - two important NOS concepts. Audrey also emphasized the perspective that the phrase "scientific thinking" more accurately describes the process of science than does the phrase "scientific method." During this phase of instruction students were in a passive mode. They arrived to class having read the handout, but Audrey still described the activity in detail. The handout was highly structured, but she noted that she did this because it was early in the course of instruction with new students.

The instruction Audrey selected to be observed was a Flinn ChemTopic ${ }^{\circledR}$ Lab entitled Observation and Experiment: Introduction to the Scientific Method. During the activity students mixed two chemicals and an indicator in a zipper-lock plastic bag and observed the chemical and physical changes that occurred. Students were to control 
mixing the chemicals in such a way as to be able to explain specifically which mixture of chemicals produced what changes and to be able to justify their conclusions.

During the course of instruction Audrey circulated around the class interacting with students in a casual manner. Early in the activity, when students asked procedural questions, Audrey provided direct answers rather than using guided questioning. Later in the activity Audrey's interactions with students shifted toward guided questioning. Near the end of the lesson, she was conferencing with a student who had not completed sufficient observations to determine whether calcium chloride is still calcium chloride when mixed with water (one of the foci of the activity). Audrey demonstrated difficulty conveying the answer to the student without being direct.

Document analysis. Students completed a lab handout including charts to help organize their activity and a series of questions to help organize their analysis (see Appendix G2). Audrey's activity was highly structured, providing explicit procedural instructions, prompting students to think of questions they might ask about their observations, and asking for description of specific descriptions of particular observations or inferences. This handout structured the activity in a way that supported students' inquiry, diminishing the need for teacher guidance.

\section{Robert}

Teacher and school description. Robert is a non-NBCT high school chemistry teacher. His entire teaching career has been at this school. Robert came to teaching from factory work when the garment factory in which he was employed moved to El Salvador. Robert has also served as the girls' basketball coach for most of his career at this school. Robert teaches in a four-person science department. His school is the only high school in 
the county. About 660 students are enrolled and taught by about 33 teachers. The student body is about $98 \%$ White and $2 \%$ African American and Hispanic. The school is not identified as a magnet school, and Title I data is not available (NCES, 2007). Robert teaches on a standard six-period schedule. The course observed was a $10^{\text {th }}$ grade Introduction to Chemistry and Physics (ICP) course. Included among the students was a special education student with an aide.

Observation description and analysis. Robert chose a lesson for observation from a set of commonly known chemistry inquiry lessons. There was no handout for students associated with the activity. Robert began the lesson by dividing the students into groups and providing them an Erlenmeyer flask in which he had mixed a solution of water, glucose, potassium hydroxide, and methylene blue indicator. The solution remained blue until shaken, at which time it became clear. After sitting a short time the solution became blue again. Students were tasked to design procedures that would conclusively determine what reaction explained the color change in the solution. Robert told students he would provide them with any equipment they desired.

During the course of the lesson, one group of students decided that blowing into the solution through a tube might provide evidence supporting their hypothesis that the presence of oxygen was the important factor causing the changing color of the solution. Instead of asking for equipment, they rolled up a piece of paper and blew through it. When Robert observed their activity he chastised the students for hazardous behavior and questioned them about components of human breath and how this information affected the quality of their arguments. During this exchange, students appeared to conclude that breath is not pure oxygen and thus, their argument was confounded by multiple variables. 
Throughout the activity Robert declined to answer students' direct questions, instead redirecting students to think differently or repeat an observation. The spectrum of student interests and abilities was significant as evidenced by their levels of interaction and behavior. Of 25 students, approximately 5 were off-task or not totally engaged. Students determined that the presence of oxygen in the solution caused the change in color but did not construct a conclusive argument for their assertion. As the end of class approached Robert called the students back together and discussed the results of their activity with them, writing their ideas on the board. As class ended he told them the correct answer which is that the indicator responds to dissolved oxygen, changing color in response to the oxygen introduced by the vigorous shaking.

Document analysis. Robert did not provide any documents for analysis.

\section{Rhonda}

Teacher and school description. Rhonda is a novice non-NBCT seventh grade teacher working in a large consolidated Kentucky urban district. The school in which Rhonda teaches enrolls about 730 students in grades six, seven, and eight and employs approximately 43 teachers. The student-body is approximately $37 \%$ African American, $61 \%$ White, and 2\% Hispanic and Asian. The school is not identified as a charter or magnet school and is recognized as a Title I school. The class chosen to be observed contained approximately 30 students constituted by a mix of ethnicities and including one special education student with an instructional aide.

District-level administration dictates that all elementary and middle school science curriculum be guided by the Foss Science Modules ${ }^{\circledR}$. This means that Rhonda has significantly less freedom to choose instructional strategies and content than did other 
teachers participating in the study. For this reason, less may be inferred from observations of Rhonda's practice than from other teachers'. This was the first year the Foss modules were being used in the school. Rhonda stated that she liked the modules because they came with all the materials she needed, relieving her of the burden of purchasing them with her own funds. In addition she shared that the modules included lots of good inquiry activities and were of high interest to her students.

Observation description and analysis. The lesson Rhonda chose to be observed occurred at the beginning of a unit on chemical change. In it students were tasked to recreate a mystery powder that consisted of a mixture of household chemicals. The day's lesson began as students were asked to write a short description of a procedure that would enable identification of the powder. Students were clustered in groups of three or four. One member from each group went to get supplies including (1) the mystery mixture, (2) vials of potential constituent powders, (3) a tray on which the test reactions were to take place, and (4) an eye dropper which was to be used to deposit water on each test mixture. Students observed the reaction of the unknown powder with water, looking for fizzing or other indications of chemical change. They then mixed known powders methodically, comparing each reaction with water to the reaction of the unknown.

Rhonda circulated continually throughout the procedure, guiding students without providing overt instruction. During the class period no direct instruction describing how to control variables was provided. By the end of class most groups had correctly identified the parts of the mixture, but students had employed what appeared to a guessand-check method rather than methodically observing and varying their mixtures. Rhonda did not engage students in discussions requiring them to justify their assertions. 
Document analysis. The activity was structured by a handout including charts on which to record results and a short list of open-ended questions (see Appendix G3). The presence of blank tables and short open-ended questions asking for observations and inferences supported effective inquiry instruction but did not provide sufficient scaffolding to move students from their standard guess-and-check mode of thought toward a more methodical control of variables without the intervention of a teacher. Jackie

Teacher and school description. Jackie is a non-NBCT middle school life science teacher. She teaches seventh grade at a middle school situated in a small rural Kentucky community. Her school enrolls about 430 students and employs 23 teachers creating a student teacher ratio of approximately 19:1. The student body is comprised of approximately 97\% White, $2 \%$ African American, and 1\% Hispanic students in grades six through eight. This school is neither a charter nor magnet school, and is not designated as a Title I school (NCES, 2007).

Observation description and analysis. The class selected for observation contained 22 students equally distributed between boys and girls and representing a range of achievement levels. The lesson Jackie chose to be observed was a small-group activity in which two students in the group were selected to be "parents" and another one or two students played the role of a child. The parents chose traits for themselves and then determined the traits inherited by the child using a Punnett square. Jackie provided explicit instruction regarding the choices she thought students should make. Student choice only existed to the extent that they chose their traits and chose the materials with which to make the poster which was the work-product for the lesson. This lesson was 
characterized by the limited extent to which science inquiry could be used in its characterization.

Document analysis. The documents Jackie presented for this activity included a handout from the previous day that structured student activity flipping coins to determine which genotype and phenotype describing various traits a "smiley face" child would inherit from his or her parents (see Appendix G4). After all traits had been determined, the student was prompted to draw the smiley face described by traits he had determined.

The document associated with the observed lesson was a rubric guiding the production of the work-product for this lesson, which was a poster describing the "Smiley Face Family" produced by each group of students. Of note on this rubric is the equal weight given to Required Elements, Labels, Accuracy, Attractiveness, and Grammar. This activity did not appear to have strong potential for student science inquiry, and Jackie did not teach in a way the made use of the limited opportunity this activity provided. Kyle

Teacher and school description. Kyle is an experienced non-NBCT eighth grade teacher. He teaches in the same school with Jackie. His assignment is physical science. Kyle is a hands-on teacher who is known in the community to have energized his students to enjoy science. He frequently holds design competitions in which students attempt the build the tallest, strongest or most successful device.

Observation description and analysis. The class he chose to be observed occurred on a day during which students were constructing bridges out of balsa wood strips. Instead of designing the bridges themselves, Kyle had provided diagrams of standard 
truss structures students were to use as templates. He provided explicit instructions regarding what he thought the best methods were for students to apply as they worked. He indicated when questioned, however, willingness to allow students to utilize other patterns to build their bridge if they so wished. He indicated that triangles were the strongest shape around which to design a bridge.

The entire class period consisted of student-directed work as Kyle circulated around the room interacting with small groups of students. The level of student engagement was rather low because several students in each group remained unoccupied. Kyle recognized this as a problem but noted that dividing the class into smaller groups was not an option because he had limited equipment. The observation did not provide evidence of whether Kyle thought this was an inquiry lesson, or if it was the most inquiry-oriented lesson he could provide during the study observations window.

Document analysis. The documents Kyle provided accompanying this activity included a teacher-produced instruction sheet and a two-page reproduced handout describing acceptable construction techniques and a page of design suggestions (see Appendix G5). No instructions related to hypothesis testing, control of variables, or any other aspect of science inquiry were present either in the primary instruction or in the documents. It appeared that science inquiry practice was not a focus of this instruction; instead the focus was on bridge construction.

\section{Jennie}

Teacher and school description. Jennie was a non-NBCT sixth grade teacher with six years experience. Jennie's school enrolls about 600 students in grades six through eight. It is neither a charter nor magnet school. It is designated as a Title I school. The 
student body is approximately $97 \%$ White, and 3\% African American and Hispanic (NCES, 2007). The school operates in a populous rural Kentucky county with a large county seat and many elementary, middle and high schools.

Observation description and analysis. The lesson Jeannie selected to be observed was an activity modeling the rock cycle. Student dialogue and displays posted on a bulletin board clearly indicated that students were familiar with the rock cycle concept and vocabulary. The lesson consisted of (1) grating chocolate, white chocolate, and butterscotch chips, (2) arranging the chips in layers, (3) compressing them under a book, and then (4) heating them on a hot plate. The work product was supposed to look like sedimentary or metamorphic rock. Students had limited success producing anything that looked like rock, but their discourse did indicate that they understood the underlying concepts. During a short interview after class, Jennie stated that she didn't believe that inquiry based instruction had a valid role in middle school pedagogy.

Document analysis. The documents Jeannie provided supporting this instructional activity included an instruction sheet guiding student activity including direction about when to answer the attached questions (see Appendix G6). These questions consisted of a series of "Identify" and "Describe" questions prompting students to reflect on how their activities modeled the rock cycle. The final question asked students to identify two ways the simulation did not portray the rock cycle as it appears in nature. The science inquiry content of the documentation was consistent with the observed inquiry content of the lesson and with Jennie's shared opinion that inquiry was not an appropriate instructional tool for middle school students.

\section{Ted}


Teacher and school description. Ted is an experienced NBCT seventh grade teacher. The school in which Ted teaches enrolls about 940 students and employs approximately 46 teachers. This is not a magnet school and is not designated as a Title I school. The student body is comprised of about $97 \%$ White and 3\% African American and Hispanic students (NCES, 2007). The school operates in the large central community of a populous rural Kentucky county.

Observation description and analysis. Ted recognized that the lesson he selected to be observed did not focus on inquiry, but in his judgment it was the lesson closest to inquiry that he would be teaching during the several weeks when the observation was to occur. Ted taught life science and was teaching a unit on heredity. The lesson was an extension of instruction focusing on the Punnett square as a tool to predict inheritance patterns. The activity was a survey of students in class regarding their possession of certain genetic traits such as the ability to roll one's tongue, or taste a particular chemical. As students raised their hands in response to Ted's queries, he tallied their responses on the board. The students then calculated proportions and created bar graphs displaying the data.

Document analysis. Ted did not provide any documents for analysis.

\section{Summary}

In all cases, the high school teachers were able to schedule, design, and enact instruction that utilized student inquiry. Susan and Audrey selected lessons that focused on inquiry as content using chemistry concepts as the medium. Robert selected a lesson that focused on chemistry concepts using inquiry as the method of instruction. The two NBCTs were able to enact inquiry instruction that appeared both compelling and 
engaging to students. The non-NBCT participant was able to select a potentially engaging inquiry lesson, but did not appear to engage his class as effectively as the other two high school teachers.

The middle school teachers were very different, however. Only in the case of Rhonda, whose instruction was constrained by a standlardized district curriculum did a middle school teacher utilize inquiry instruction. In all other cases, the teachers selected lessons to be observed that utilized inquiry only minimally, if at all. Jennie's opinion that inquiry was not an appropriate mode of science instruction for middle school students may help explain some of this trend.

Ted, the single middle school NBCT who was observed, did recognize that the instruction he selected to be observed was not a good example of inquiry, but he had scheduled no other lesson that was a better example. He did not explain the reason for this circumstance. Several possible situations may explain the middle school teachers' difficulty finding an inquiry lesson during the observation period. This may be evidence of the infrequency of inquiry in their classrooms or that the units they were teaching at that time did not provide a rich content base from which to design inquiry experiences for middle school students. An alternative explanation may be that the constraints on their professional time prevented them from marshalling the resources necessary to enact an inquiry activity.

Although there were no significant differences in the number of years of experience between middle and high school teachers and between NBCTs and nonNBCTs in the teacher sample as a whole, in the observed sub-sample of teachers there clearly were differences in years of experience among the NBCTs and non-NBCTs. This 
may have been a factor contributing to the differences in practice observed between the two groups. This potential counterfactual is limited to some extent by the triangulation of these results with other quantitative findings in this study.

\section{Question 3 Findings:}

High school students who perceived that they used data for explanation more frequently displayed higher levels of science reasoning. No classroom inquiry environment factors were related to differences in their NOS understanding

Differences in student science reasoning and NOS understanding. Analyses of differences between students' scientific reasoning and NOS understanding scores were completed before exploring for any potential relationships between those student abilities and frequency of classroom behaviors. Students were grouped according to the four-level of the NBCT—school level assignment variable because important differences exist between the culture and climate of middle and high schools and between the developmental stage of middle and high school students. Table 4.8 displays the means and standard deviations of science reasoning and NOS understanding scores for students of middle school NBCTs and non-NBCTs, and students of high school NBCTs and nonNBCTs for both phase 1 and phase 2 of data collection.

Table 4.8

Means and Standard Deviations of Students' Science Reasoning and NOS Understanding Scores

\begin{tabular}{lccccc}
\hline & \multicolumn{3}{c}{ Science Reasoning } & \multicolumn{2}{c}{ NOS Understanding } \\
\cline { 2 - 6 } Student Assignment & $\mathrm{N}$ & Phase 1 & Phase 2 & Phase 1 & Phase 2 \\
\hline MS non-NBCT & 346 & $2.33(1.68)$ & $2.51(1.93)$ & $-0.37(6.49)$ & $-0.40(6.80)$ \\
MS NBCT & 153 & $2.65(1.66)$ & $2.61(1.82)$ & $1.22(6.22)$ & $0.19(5.90)$ \\
HS non-NBCT & 322 & $3.63(2.43)$ & $3.57(2.71)$ & $1.31(7.05)$ & $2.08(7.54)$ \\
HS NBCT & 273 & $4.57(2.30)$ & $4.58(2.43)$ & $1.79(7.01)$ & $2.12(7.07)$ \\
\hline
\end{tabular}


Figure 4.1 displays the mean science reasoning scores for each group of students for phase 1 and phase 2 of data collection. A clear pattern distinguishes the science reasoning scores of students by group but no difference is evident between phases of data collection. Of particular note is the difference between students of high school NBCTs and non-NBCTs. Because science reasoning is developmentally linked (meaning older students perform at higher levels than younger students) one would expect to see difference between middle and high school students, but detecting a difference correlated with teacher NBCT status between students drawn from the same developmental group raises interesting questions.

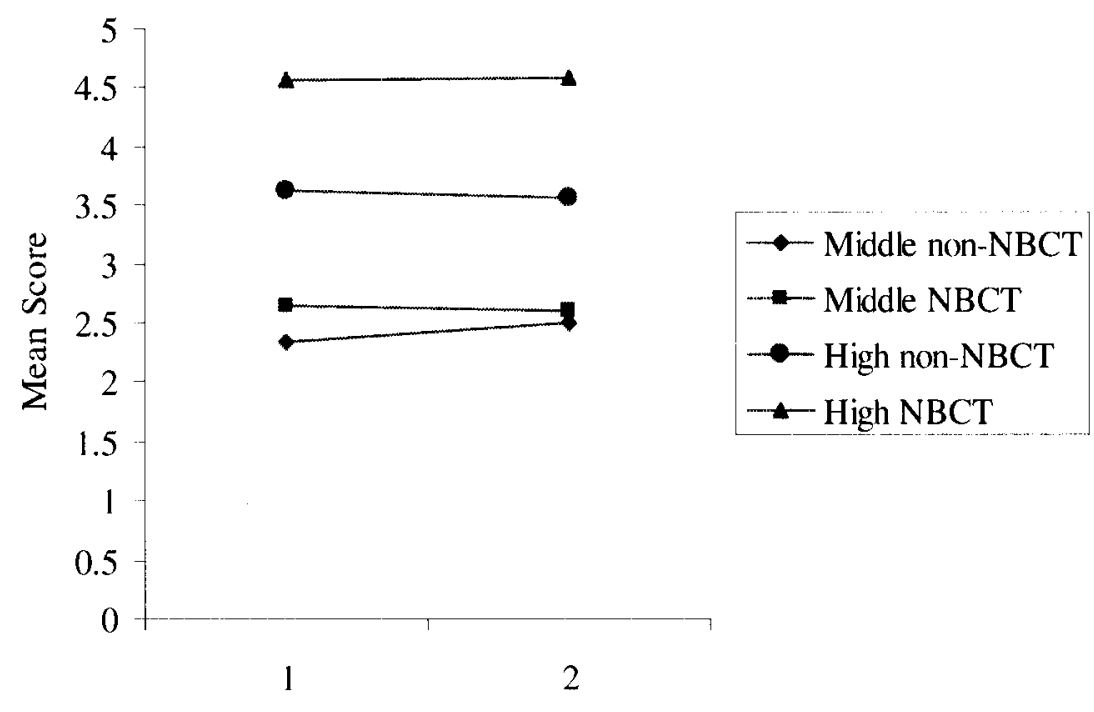

Assessment Phase

Figure 4.1. Graph of Student Science Reasoning Score Means from Phase 1 and Phase 2 of Data Collection.

Figure 4.2 displays the mean NOS understanding scores for each group of students for phase 1 and phase 2 of data collection. Some growth appears to have occurred for high school students. Students of middle school non-NBCTs are distinguished by their relatively uninformed views of NOS, but students of middle school 
NBCTs exhibit a puzzling trend. Figure 4.2 should be interpreted in light of the large standard deviations present in all student NOS understanding data (see Table 4.1).

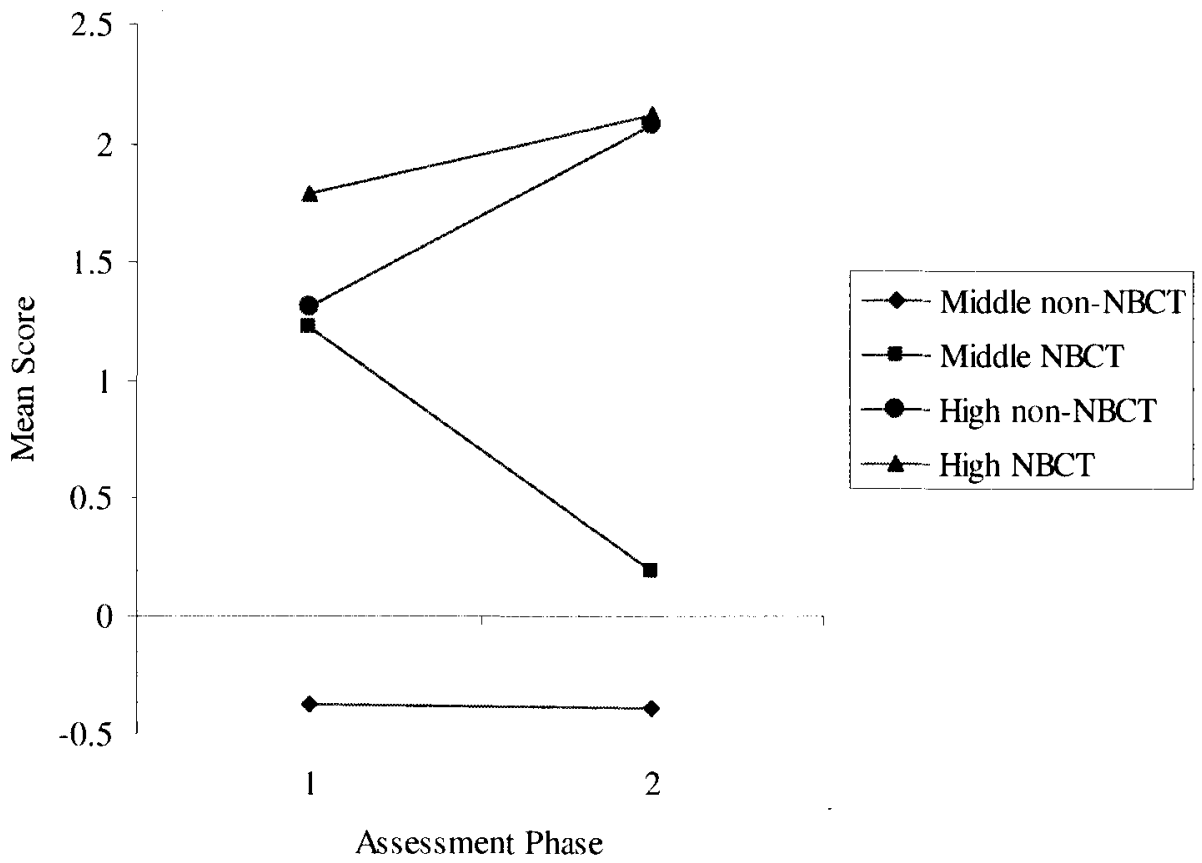

Figure 4.2. Graph of Student NOS Understanding Score Means from Phase 1 and Phase 2 of Data Collection

ANOVA was used to compare student science reasoning and NOS understanding between levels of this variable (Table 4.9). Significant differences were detected at the $p$ $<0.001$ level between levels of the NBCT - school level assignment variable. Post hoc analyses of the ANOVA of science reasoning scores indicated that significance was contributed by differences between students of high school NBCTs and all others and between students of high school non-NBCTs and all middle school students, but that students of middle school NBCTs and non-NBCTs were not significantly different from each other. Post hoc analyses of the ANOVA of NOS understanding scores indicated that significance was contributed by differences between high school students and middle school students regardless of the NBCT status of their teachers. 
Table 4.9

One-Way Analyses of Variance of Effects of Student Assignment on Phase 2 Science Reasoning and NOS Understanding

\begin{tabular}{rcccc}
\hline Variable and Source & df & SS & MS & F \\
\hline Science Reasoning & & & & \\
Between Groups & 3 & 756.491 & 252.164 & $47.831^{* * *}$ \\
Within Groups & 1090 & 5746.424 & 5.272 & \\
NOS Understanding & & & & \\
Between Groups & 3 & 1498.304 & 499.435 & $10.238^{* * *}$ \\
Within Groups & 1090 & 53671.310 & 48.783 & \\
\hline$* * * \mathrm{p}<0.001$ & & & &
\end{tabular}

Relationship of student science reasoning and NOS understanding with teacher

variables. Multivariate analysis of variance (MANOVA) was performed on the second phase student assessment data using teacher NBCT status, experience level, and noneducation degree level as independent variables with student grade level as a covariate. Student science reasoning and nature of science understanding were dependent variables. Substantial variability in cell size existed so the multivariate test would be sensitive to non-normality of the data (Stevens, 2002). Levene's test for equality of variances was $22.597(\mathrm{df}=3,1090, \mathrm{p}<0.001)$ for the science reasoning assessment, and was $4.299(\mathrm{df}$ $=3,1090, \mathrm{p}<0.01)$ indicating that a violation of the equality of variances assumption existed for both data sets. The normality of the student science reasoning and nature of science understanding data was calculated with the Shapiro-Wilk normality test statistic. For the science reasoning data the statistic was $0.921(\mathrm{df}=1486, \mathrm{p}<0.001)$, and for nature of science understanding it was $0.978(\mathrm{df}=1486, \mathrm{p}<0.001)$ indicating that both distributions violated the normality assumption. Examination of histograms of the data indicated that student science reasoning measurements were positively skewed, and nature of science understanding was leptokurtic. In addition, Box's test for equality of covariance matrices revealed significant differences in the variability between both 
middle and high school groups. For these reasons the significance of the test should be interpreted cautiously.

High school student achievement and teacher characteristics. The MANOVA for high school students indicated statistically significant effects for teacher NBCT status, experience level, NBCT status - teacher non-education degree interaction, and teacher non-education degree - experience level interaction (Table 4.10).

Table 4.10

Multivariate and Univariate Analyses of Variance F Ratios for High School Student NBCT Status, Degree, and Experience Effects on Science Reasoning and NOS Understanding

\begin{tabular}{lccc}
\hline & & \multicolumn{2}{c}{ ANOVA } \\
\cline { 3 - 4 } \multicolumn{1}{c}{ Variable } & MANOVA & Science Reasoning & NOS Understanding \\
\hline NBCT (N) & $11.710^{\mathrm{a} * * * *}$ & $3.644^{\mathrm{c}}$ & $22.737^{\mathrm{c} * * *}$ \\
Degree (D) & $1.823^{\mathrm{a}}$ & $0.271^{\mathrm{c}}$ & $2.799^{\mathrm{c}}$ \\
Experience (E) & $15.475^{\mathrm{b} * * *}$ & $24.978^{\mathrm{c} * * *}$ & $13.522^{\mathfrak{c}^{*} * * *}$ \\
N x D & $17.890^{\mathrm{a} * * *}$ & $22.486^{\mathrm{c} * * *}$ & $21.472^{\mathrm{c}^{*} * *}$ \\
N x E & $0.670^{\mathrm{a}}$ & $0.759^{\mathrm{c}}$ & $0.298^{\mathrm{c}}$ \\
D x E & $13.006^{\mathrm{b} * * *}$ & $22.415^{\mathrm{c} * * *}$ & $9.273^{\mathrm{c} * * *}$ \\
\hline
\end{tabular}

Note. F ratios are Wilk's approximations of Fs.

${ }^{a}$ Multivariate $\mathrm{df}=2,585 .{ }^{\mathrm{b}}$ Multivariate $\mathrm{df}=4,1170 .{ }^{\mathrm{c}}$ Univariate $\mathrm{df}=1,595$. p $<0.01 . * \mathrm{p}<0.05 . * * \mathrm{p}<0.01 .{ }^{* * *} \mathrm{p}<0.001$.

The significance at the high school level was created by differences in both student science reasoning and nature of science understanding. Examination of the estimated marginal means indicated that significant effect of teacher NBCT status on science reasoning benefited students' of NBCTs, while the significant effect of NBCT status on NOS understanding benefited students' of non-NBCT's. Otherwise, increased education and experience benefited students in the expected direction.

Middle school student achievement and teacher characteristics. MANOVA was completed for middle school students measuring differences in science reasoning and NOS understanding related to teacher NBCT status, non-education degree, and 
experience level. It indicated statistically significant main effects for teacher non-

education degree, and teacher experience level (Table 4.11).

Table 4.11

Multivariate and Univariate Analyses of Variance F Ratios for Middle School Student NBCT Status, Degree, and Experience Effects on Science Reasoning and NOS Understanding

\begin{tabular}{lccc}
\hline & & \multicolumn{2}{c}{ ANOVA } \\
\cline { 3 - 4 } \multicolumn{1}{c}{ Variable } & MANOVA & Science Reasoning & NOS Understanding \\
\hline NBCT (N) & $0.437^{\mathrm{a}}$ & $0.680^{\mathrm{c}}$ & $0.310^{\mathrm{c}}$ \\
Degree (D) & $6.423^{\mathrm{b}} * * *$ & $11.588^{\mathrm{c} * * *}$ & $0.756^{\mathrm{c}}$ \\
Experience (E) & $3.796^{\mathrm{b} * *}$ & $5.282^{\mathrm{c}} * *$ & $1.751^{\mathrm{c}}$ \\
N x D & $0.381^{\mathrm{a}}$ & $0.747^{\mathrm{c}}$ & $0.000^{\mathrm{c}}$ \\
N x E & $1.211^{\mathrm{a}}$ & $2.330^{\mathrm{c}}$ & $0.008^{\mathrm{c}}$ \\
D x E & $0.755^{\mathrm{b}}$ & $1.415^{\mathrm{c}}$ & $0.200^{\mathrm{c}}$ \\
\hline
\end{tabular}

Note. F ratios are Wilk's approximations of Fs.

${ }^{a}$ Multivariate $\mathrm{df}=2,488 .{ }^{\mathrm{b}}$ Multivariate $\mathrm{df}=4,978 .{ }^{\mathrm{c}}$ Univariate $\mathrm{df}=1,499$. $\mathrm{p}<0.01{ }^{*} \mathrm{p}<0.05$. ** $\mathrm{p}<0.01$. $^{* * *} \mathrm{p}<0.001$.

The significant effects detected in the multivariate test were created by differences on student science reasoning alone, with no effect on student nature of science understanding. Examination of the marginal means for this student group indicated increasing teacher non-education degree was associated with lower science reasoning skill while increasing experience level was associated with higher student science reasoning. Sample size differences may have been associated with these counterintuitive results as there were significant differences (ratio $>1.5 / 1$ ) between the sizes of middle school teacher groups organized by both teacher non-education degree and experience rank.

Student Gains. All differences in student performance on the science reasoning and NOS assessments were detected in the first phase data measured in October. In order to detect if any change in student understanding was attributable to teacher characteristics, MANOVA was completed using the difference between phase 2 and 
phase 1 science reasoning total score and NOS understanding total score as dependent variables, and teacher NBCT--middle/high school status, experience level, and noneducation degree, as independent variables with grade as a covariate. Examination of the estimated marginal means tables indicated in all cases zero gain was in the $95 \%$ confidence interval, indicating no growth in either science reasoning or NOS understanding. This means that there were no significant differences in scores related to teacher status or environment variables between the first and second rounds of data collection.

\section{Differences in Teacher Practice}

Having established that differences existed between students' of NBCTs and their non-NBCT counterparts and between middle and high school classrooms, it is important to attempt to link differences in teacher practice that may be associated with these differences in student achievement. To do this, the sub-scores defined by the four classroom inquiry environment factors described earlier in this chapter and student gradelevel were regressed against the science reasoning total score and the NOS understanding total score. Prior to calculating regression equations however, missing data were imputed with the series mean using the SPSS $14^{(}$Replace Missing Values routine (SPSS, 2005). Again, for this analysis, the data were divided into groups corresponding to the two levels of NBCT status and two levels of middle/high school assignment.

Students of middle school non-NBCTs. The intact data were analyzed prior to disaggregation into groups to determine if assumptions relating to the structure of the data were valid. The residuals were examined for each analysis to evaluate the normality and homoscedasticity assumptions. The normality plot of expected cumulative 
probability vs. the observed cumulative probability was nearly linear along the diagonal, indicating the residuals were normally distributed. Partial regression plots were randomly scattered, which reflected lack of violation of the homogeneity assumption. Additionally, neither influential data points (values of Cook's D ranged from 0.00 to 0.032 ), nor multicollinearity (the Variance Inflation Factor displayed values from 1.085 to 2.898 ) were observed in the data (Stevens, 2002).

The four classroom inquiry environment factors were entered into the equation relating classroom inquiry behaviors with student science reasoning skills, the significance of which was $p=0.112$. The adjusted $R^{2}$-value was 0.016 , indicating that only about $2 \%$ of the variance in student scientific reasoning was explained by the linear combination of the behaviors defined by the four classroom inquiry environment factors and student grade level. The standardized regression coefficients indicated that GradeLevel was a significant predictor at the $\mathrm{p}<0.05$ level, and Approach to Science negatively predicted student science reasoning at the $\mathrm{p}<0.01$ level (Table 4.12). Similar analysis of the relationship between NOS understanding (SUSSI total score) indicated that although the important assumptions were met by the data, no significant relationship existed between the dependent and independent variables except between student's grade in school.

Table 4.12

Simultaneous Regression of Science Reasoning on Inquiry Environment Factors and Students' Grade Level in School

\section{Corr}

with

\begin{tabular}{llllllll} 
Predictors & $\mathrm{R}$ & $\mathrm{R}^{2}$ & Adj. $\mathrm{R}^{2}$ & $\mathrm{~B}$ & $\mathrm{SEB}$ & $\beta$ & Score \\
\hline
\end{tabular}

$\begin{array}{lcccc} & \text { Students of Middle School non-NBCTs }(N=250) & & \\ \text { Investigation } & 0.032 & 0.017 & 0.205^{\dagger} & 0.012 \\ \text { Use of Data } & 0.023 & 0.036 & 0.052 & 0.014 \\ \text { Science Dialogue } & -0.007 & 0.015 & -0.041 & -0.041\end{array}$


Approach to Science

$\begin{array}{llll}-0.095 & 0.037 & -0.255^{* *} & -0.111\end{array}$

Grade in School

$0.090 \quad 0.140$

0.042

0.062

$0.189 \quad 0.036 \quad 0.016$

\begin{tabular}{lcccc}
\hline & Students of High School non-NBCTs $(N=276)$ & & \\
Investigation & 0.017 & 0.025 & 0.073 & 0.037 \\
Use of Data & 0.087 & 0.053 & $0.124^{\dagger}$ & 0.107 \\
Science Dialogue & -0.021 & 0.021 & -0.089 & -0.015 \\
Approach to Science & -0.041 & 0.055 & -0.069 & 0.002 \\
Grade in School & 0.458 & 0.206 & $0.143^{*}$ & 0.142
\end{tabular}

$0.193 \quad 0.037 \quad 0.017$

\begin{tabular}{lcccc}
\hline & Students of Middle School NBCTs $(N=116)$ & & \\
Investigation & -0.017 & 0.024 & -0.104 & 0.016 \\
Use of Data & 0.031 & 0.062 & 0.058 & 0.044 \\
Science Dialogue & 0.032 & 0.023 & 0.183 & 0.109 \\
Approach to Science & -0.024 & 0.051 & -0.055 & -0.010 \\
Grade in School & 0.269 & 0.352 & 0.075 & 0.074
\end{tabular}

$\begin{array}{lll}0.164 & 0.027 & -0.017\end{array}$

Students of High School NBCTs $(N=245)$

$\begin{array}{lcccc}\text { Investigation } & 0.018 & 0.023 & 0.079 & 0.204 \\ \text { Use of Data } & 0.123 & 0.054 & 0.176^{*} & 0.235 \\ \text { Science Dialogue } & -0.002 & 0.019 & -0.011 & 0.154 \\ \text { Approach to Science } & 0.028 & 0.048 & 0.050 & 0.165 \\ \text { Grade in School } & 0.023 & 0.156 & 0.010 & 0.019\end{array}$

$\begin{array}{lll}0.253 & 0.064 \quad 0.045^{* *}\end{array}$

$\mathrm{p}<0.10,{ }^{*} \mathrm{p}<0.05,{ }^{* *} \mathrm{p}<0.01$

Students of high school non-NBCTs. Examination of the normality plot of expected cumulative probability vs. the observed cumulative probability was nearly linear along the diagonal, indicating the residuals were normally distributed. Partial regression plots were randomly scattered, which reflected lack of violation of the homogeneity assumption. Additionally, neither influential data points (values of Cook's

D ranged from 0.00 to 0.060 ), nor multicollinearity (the Variance Inflation Factor displayed values from 1.020 to 2.644) were observed in the data (Stevens, 2002).

Again, four classroom inquiry environment factors were entered into the equation relating classroom inquiry behaviors with student science reasoning skills, the 
significance of which was $p=0.102$. The adjusted $R^{2}$-value was 0.017 indicating that about $2 \%$ of the variance in student scientific reasoning was explained by the four ESIS factors and student grade level. The standardized regression coefficients indicated that Grade-Level $(\mathrm{p}<0.05)$ and Use of Data $(\mathrm{p}<0.10)$ were significant predictors of student scientific reasoning. Similar analysis of the relationship between NOS understanding indicated that although the important assumptions were met by the data, no significant relationship existed between the dependent and independent variables.

Students of middle school NBCTs. Examination of the normality plot of expected cumulative probability vs. the observed cumulative probability was nearly linear along the diagonal, indicating the residuals were normally distributed. Partial regression plots were randomly scattered, which reflected lack of violation of the homogeneity assumption. Additionally, neither influential data points (values of Cook's D ranged from 0.00 to 0.060 ), nor multicollinearity (the Variance Inflation Factor displayed values from 1.101 to 2.330 ) were observed in the data (Stevens, 2002).

When the four classroom inquiry environment factors were entered into the equation relating classroom inquiry behaviors with student science reasoning skills, the significance of which was $p=0.693$. The adjusted $\mathrm{R}^{2}$-value was -0.017 indicating that about $2 \%$ of the variance in student scientific reasoning was explained by the four ESIS factors and student grade level. The standardized regression coefficients indicated that none of the factors entered were significant predictors of student scientific reasoning. Similar analysis of the relationship between NOS understanding indicated that although the important assumptions were met by the data, no significant relationship existed between the dependent and independent variables. 
Students of high school NBCTs. Examination of the normality plot of expected cumulative probability vs. the observed cumulative probability was nearly linear along the diagonal, indicating the residuals were normally distributed. Partial regression plots were randomly scattered, which reflected lack of violation of the homogeneity assumption. Additionally, neither influential data points (values of Cook's D ranged from 0.00 to 0.035 ), nor multicollinearity (the Variance Inflation Factor displayed values from 1.026 to 2.672) were observed in the data (Stevens, 2002).

When the four classroom inquiry environment factors were entered into the equation relating classroom inquiry behaviors with student science reasoning skills, the significance of which was $p=0.007$. The adjusted $R^{2}$-value was 0.045 indicating that about $5 \%$ of the variance in student scientific reasoning was explained by the four ESIS factors and student grade level. The standardized regression coefficients indicated that Use of Data was a significant predictor of student scientific reasoning. Similar analysis of the relationship between NOS understanding indicated that although the important assumptions were met by the data, no significant relationship existed between the dependent and independent variables.

\section{Summary}

Student perceptions of both high school NBCTs' and non-NBCTs' Use of Data in their classroom appeared to be related to their scientific reasoning skills. No other factor from the ESIS survey was significantly related to either science reasoning or understanding the nature of science. Two results were surprising and merit further investigation. Approach to Science may have been negatively correlated with science reasoning for students of middle school non-NBCTs, and grade was a significant 
predictor of science reasoning skills only for students of high school non-NBCTs. The persistence of patterns in student and teacher NOS understanding raise questions regarding the nature of NOS understanding and whether it is developmentally linked.

\section{Question 4 Findings:}

Factors Describing Teacher Background, School Environment were Important to the

\section{Existence of Classroom Inquiry Environments}

This analysis was completed correlating NBCT and non-NBCT responses to survey questions with the aggregate classroom science inquiry environment ratings provided by their students. For this reason the teacher data set was used. This limited the number of participants for analysis, preventing the use of the four-level NBCT statusmiddle/high school assignment variable for grouping responses. Instead, two parallel analyses were completed comparing middle and high school groups, and NBCT and nonNBCT groups.

\section{Middle and High School Groups}

Teacher characteristics. Table 4.13 displays the correlations of middle school teacher ratings of various experiential variables with the mean student responses to the four ESIS factor sub-scores described previously. Table 4.14 displays the same relationships for the high school teachers and their students. Significant relationships with students' perceptions of classroom inquiry environment were detected in the middle school teacher background data (Table 4.13), but not in the high school data (Table 4.14). Specifically, middle school teachers' Collaboration with Other Science Teachers was positively correlated with their students' perceptions of classroom Investigation activities, and activities related to Approach to Science. Attendance at science education workshops 
in the last five years seemed to be equally important, correlating significantly with students' perceptions of Science Dialogue and Approach to Science.

Table 4.13

Correlations of Middle School Teacher Characteristics with Classroom Science Inquiry Factors

\begin{tabular}{|c|c|c|c|c|}
\hline $\begin{array}{l}\text { Pearson Correlations } \\
\mathrm{N}=22\end{array}$ & Investigation & $\begin{array}{l}\text { Use of } \\
\text { Data }\end{array}$ & $\begin{array}{l}\text { Science } \\
\text { Dialogue }\end{array}$ & $\begin{array}{c}\text { Approach } \\
\text { to } \\
\text { Science }\end{array}$ \\
\hline $\begin{array}{l}\text { Collaboration with other science } \\
\text { teachers }\end{array}$ & $0.539 * *$ & $0.420^{\dagger}$ & 0.032 & $0.620 * *$ \\
\hline Number of graduate courses & 0.093 & 0.287 & -0.003 & 0.191 \\
\hline $\begin{array}{l}\text { College science course inquiry } \\
\text { emphasis }\end{array}$ & 0.271 & -0.040 & -0.074 & 0.278 \\
\hline Science ed. workshops last 5 years & 0.200 & $0.412^{\dagger}$ & $0.454 *$ & $0.462 *$ \\
\hline $\begin{array}{l}\text { Conducted or assisted scientific } \\
\text { research }\end{array}$ & 0.324 & 0.115 & 0.154 & $\mathbf{0 . 3 9 0 ^ { \dagger }}$ \\
\hline NSES familiarity & 0.198 & 0.054 & 0.320 & $0.471 *$ \\
\hline KPOS familiarity & -0.271 & -0.289 & $-0.434 *$ & -0.052 \\
\hline KY Core Content familiarity & -0.271 & -0.278 & -0.345 & -0.264 \\
\hline
\end{tabular}

$\mathrm{p}<0.10{ }^{*} \mathrm{p}<0.05$. $^{* *} \mathrm{p}<0.01$.

Table 4.14

Correlations of High School Teacher Characteristics with Classroom Science Inquiry Factors

\begin{tabular}{|c|c|c|c|c|}
\hline $\begin{array}{l}\text { Pearson Correlations } \\
\mathrm{N}=28\end{array}$ & Investigation & $\begin{array}{l}\text { Use of } \\
\text { Data }\end{array}$ & $\begin{array}{l}\text { Science } \\
\text { Dialogue }\end{array}$ & $\begin{array}{l}\text { Approach } \\
\text { to } \\
\text { Science }\end{array}$ \\
\hline $\begin{array}{l}\text { Collaboration with other science } \\
\text { teachers }\end{array}$ & 0.219 & 0.152 & 0.071 & 0.019 \\
\hline Number of graduate courses & 0.146 & 0.260 & 0.159 & 0.151 \\
\hline $\begin{array}{l}\text { College science course inquiry } \\
\text { emphasis }\end{array}$ & 0.107 & 0.032 & 0.007 & -0.028 \\
\hline Science ed. workshops last 5 years & 0.274 & 0.237 & 0.155 & 0.252 \\
\hline $\begin{array}{l}\text { Conducted or assisted scientific } \\
\text { research }\end{array}$ & -0.162 & 0.012 & -0.119 & -0.175 \\
\hline NSES familiarity & 0.099 & 0.093 & -0.057 & 0.197 \\
\hline KPOS familiarity & 0.080 & 0.027 & -0.026 & 0.149 \\
\hline KY Core Content familiarity & -0.260 & $-0.333^{\dagger}$ & -0.290 & -0.197 \\
\hline
\end{tabular}
$\mathrm{p}<0.10$.

Independent samples t-tests were performed comparing middle and high school teacher responses to these questions in order to evaluate the significance pattern 
differences between the two groups. These tests evaluated whether the actual frequencies of activities were different or if only their consequent effect on student perceptions was different. No significant differences were detected between the middle and high school teachers on these variables. These results indicated that these middle and high school teachers had similar responses to these questions describing their pertinent professional demographic variables. In spite of this similarity, teacher collaboration and participation in science education workshops were correlated with middle school student perceptions of science classroom inquiry environments but not with high school student perceptions. The presence of correlations for middle school teacher but not for high school teachers may indicate the presence of differences in these characteristics not measured by these questions, or it may indicate the interaction of these characteristics with other unmeasured school, teacher, or student characteristics.

Teaching contexts. Teaching contexts were operationalized on the ESIS teacher survey as items describing factors that determine curriculum and factors that inhibit classroom inquiry. Correlations between teacher responses to these items and student perceptions of classroom inquiry practice were evaluated to measure relationship between the constructs. Middle school students reported lower levels of classroom inquiry practice when teachers reported higher priority for state and national standards in determining their curriculum. These students also reported higher levels of inquiry when their teachers reported higher priority of the textbook in determining curriculum. Student perceptions of classroom science dialogue were positively correlated with teachers' perception that Nothing Limits Inquiry and negatively correlated to teachers' perceptions of Lack of Experience with Inquiry. Of the four factors describing middle school student perceptions 
of classroom inquiry environments, Science Dialogue was the most sensitive to teaching contexts (Table 4.15).

Table 4.15

Correlations of Middle School Teaching Context with Classroom Science Inquiry Factors

\begin{tabular}{lcccc}
\hline Polyserial Correlation & Inves- & Use of & Science & Approach to \\
$\mathrm{N}=22$ & tigation & Data & Dialogue & Science \\
\hline
\end{tabular}

\begin{tabular}{|c|c|c|c|c|}
\hline \multicolumn{5}{|c|}{ Factors Determining Curriculum } \\
\hline District curriculum & 0.124 & 0.040 & 0.08 & 0.406 \\
\hline State or national standards & $-0.279^{\dagger}$ & $-0.307^{\dagger}$ & $-0.334 *$ & $-\mathbf{0 . 3 5 1} 1^{\dagger}$ \\
\hline Textbook & $0.262 *$ & $0.323 *$ & $\mathbf{0 . 2 3 1}{ }^{\dagger}$ & 0.291 \\
\hline Available instructional materials & -0.089 & -0.111 & -0.089 & -0.116 \\
\hline Guidance from principal & -0.141 & -0.089 & -0.373 & $-0.474 \dagger$ \\
\hline Other teachers' input & 0.111 & 0.216 & 0.198 & -0.134 \\
\hline Other & -0.454 & -0.341 & -0.266 & -0.134 \\
\hline \multicolumn{5}{|c|}{ Factors Limiting Inquiry } \\
\hline Nothing limits & 0.05 & 0.504 & $0.436^{* *}$ & 0.173 \\
\hline Lack of experience /awareness & -0.668 & -0.595 & $-0.667 * *$ & -0.738 \\
\hline Reluctant to give up central role & -0.253 & -0.225 & -0.34 & -0.468 \\
\hline There isn't enough time & 0.582 & 0.542 & 0.409 & 0.399 \\
\hline Have tried it unsuccessfully & -0.331 & $-0.474^{\dagger}$ & -0.392 & -0.645 \\
\hline Doubt student inquiry capability & 0.230 & 0.213 & 0.263 & 0.240 \\
\hline Concern about discipline, etc. & -0.345 & -0.165 & -0.470 & -0.410 \\
\hline Insufficient time and support & 0.207 & 0.092 & 0.148 & 0.126 \\
\hline Doesn't have materials & -0.174 & -0.239 & -0.23 & -0.019 \\
\hline Lack of community support & -0.417 & -0.289 & -0.276 & -0.233 \\
\hline Other & -0.314 & -0.223 & $-0.414^{\dagger}$ & -0.304 \\
\hline
\end{tabular}

$\mathrm{p}<0.10 .^{*} \mathrm{p}<0.05 .{ }^{* *} \mathrm{p}<0.01$.

High school students displayed a different pattern of correlations with their teachers' perceptions of their teaching context. There was no significant correlation with state or national standards. Teachers' perception that their textbook was important in determining curriculum was negatively correlated with students' perceptions of Science Dialogue. Guidance from the principal in determining curriculum was negatively correlated with students' perceptions of Investigation. Teachers' responses classified as Other were positively correlated to student perceptions of approach to science. These responses are displayed in (Table 4.16). 
Table 4.16

Correlations of High School Teaching Context with Classroom Science Inquiry Factors

\begin{tabular}{|c|c|c|c|c|}
\hline $\begin{array}{l}\text { Polyserial Correlation } \\
\mathrm{N}=28\end{array}$ & $\begin{array}{l}\text { Inves- } \\
\text { tigation }\end{array}$ & $\begin{array}{l}\text { Use of } \\
\text { Data }\end{array}$ & $\begin{array}{c}\text { Science } \\
\text { Dialogue }\end{array}$ & $\begin{array}{l}\text { Approach to } \\
\text { Science }\end{array}$ \\
\hline \multicolumn{5}{|c|}{ Factors Determining Curriculum } \\
\hline District curriculum & 0.426 & -0.020 & 0.033 & 0.142 \\
\hline State or national standards & -0.28 & 0.314 & -0.052 & 0.31 \\
\hline Textbook & -0.61 & -0.525 & $-0.490 * *$ & -0.207 \\
\hline Available instructional materials & -0.095 & -0.035 & 0.269 & 0.533 \\
\hline Guidance from principal & $-0.531 * *$ & -0.500 & -0.048 & -0.432 \\
\hline Other teachers' input & 0.466 & 0.015 & 0.234 & -0.042 \\
\hline Other & 0.094 & 0.34 & 0.009 & $0.236 * *$ \\
\hline \multicolumn{5}{|c|}{ Factors Limiting Inquiry } \\
\hline Nothing limits & -0.244 & -0.252 & -0.012 & -0.082 \\
\hline Lack of experience /awareness $\mathfrak{E}$ & -0.178 & -0.267 & -0.308 & -0.63 \\
\hline There isn't enough time & 0.212 & 0.241 & 0.106 & 0.269 \\
\hline Doubt student inquiry capability & 0.248 & 0.719 & 0.211 & -0.215 \\
\hline Concern about discipline, etc. & 0.411 & 0.163 & 0.426 & $-0.089 * *$ \\
\hline Insufficient time and support & -0.002 & -0.275 & 0.010 & -0.426 \\
\hline Disagrees with inquiry necessity & 0.48 & 0.999 & 0.417 & $0.147 * *$ \\
\hline Doesn't have materials & -0.189 & -0.252 & 0.409 & 0.088 \\
\hline Lack of community support & -0.735 & -0.377 & -0.106 & -0.362 \\
\hline Other & -0.622 & -0.448 & 0.112 & -0.52 \\
\hline
\end{tabular}

$\mathrm{p}<0.10$. $^{*} \mathrm{p}<0.05 .{ }^{* *} \mathrm{p}<0.01$.

Of the nine teachers who offered "Other" responses, five cited personal

experience, and three cited college or AP curriculum requirements as factors determining curriculum (Table 4.17). Among the Factors Limiting Inquiry, Concern about Discipline was negatively correlated to Approach to Science while Disagreement with the Necessity of Inquiry Instruction was positively correlated with it (Table 4.16).

Table 4.17

Teacher Constructed Responses to the "Other" Category

\begin{tabular}{lll}
\hline School Level & N & Description \\
\hline & \multicolumn{2}{c}{ Middle School $(N=22)$} \\
Factors Impacting & 15 & Blank \\
Science Curriculum & 1 & Determined by curriculum specialist \\
& 1 & How core content is split among science teachers \\
& 1 & Kentucky Core Content \\
& 1 & School-wide curriculum map \\
& 1 & Foss Science Modules \\
& 2 & Student interest
\end{tabular}




\begin{tabular}{lll} 
Factors Impeding & 14 & Blank \\
Inquiry Instruction & 4 & High-stakes testing pressure for content coverage \\
& 1 & Lack of district support \\
& 1 & Monetary issues \\
& 1 & Student apathy \\
& 1 & Teacher professional judgment \\
\hline & & High School $(N=28)$ \\
Factors Impacting & 17 & Blank \\
Science Curriculum & 1 & AP College Board requirements \\
& 2 & Teacher research knowledge/experience \\
& 2 & What colleges want \\
& 3 & Teacher professional judgment \\
& 1 & Required student research project \\
Factors Impeding & 20 & Blank \\
Inquiry Instruction & 1 & Having to re-teach prior content \\
& 1 & Works to add inquiry to every unit \\
& 1 & Lack of space \\
& 1 & Lack of time to prepare for inquiry instruction \\
& 1 & Lack of money \\
& 2 & Not trained in content area being taught \\
& 1 & Required student research project \\
\hline
\end{tabular}

NBCTs and non-NBCTs.

Teacher characteristics. A different pattern emerged when responses were disaggregated according to NBPTS certification status. In order for a polyserial correlation to be calculated by Lisrel, its algorithm must create an asymptotic covariance matrix that converges. When the data were split according to NBPTS certification status, neither sub-set of data produced an asymptotic covariance matrix that converged. Because the Lisrel 8.80 (Jöreskog \& Sörbom, 2006) could not compute the polyserial correlations for the data split by NBPTS certification status, Pearson correlations were calculated instead. This was acceptable because Pearson correlations underestimate the size of the correlation between ordinal variables, and therefore are conservative estimators (Byrne, 1998). 
The perception of the students of non-NBCTs' regarding classroom investigation was positively correlated to their teacher's perception of frequent collaboration with other science teachers. Additionally, when teachers reported that Kentucky Core Content familiarity was important to their curriculum design, their students perceived a decrease in classroom Use of Data and Science Dialogue inquiry environment factors (Table 4.18).

Table 4.18

Correlations of non-NBCT Professional Characteristics with Classroom Science Inquiry Factors

\begin{tabular}{lcccc}
\hline $\begin{array}{l}\text { Pearson Correlations } \\
\mathrm{N}=31\end{array}$ & $\begin{array}{c}\text { Use of } \\
\text { Data }\end{array}$ & $\begin{array}{c}\text { Science } \\
\text { Dialogue }\end{array}$ & $\begin{array}{c}\text { Approach } \\
\text { to Science }\end{array}$ \\
\hline $\begin{array}{l}\text { Collaboration with other science } \\
\text { teachers }\end{array}$ & $\mathbf{0 . 4 3 0 * *}$ & 0.190 & 0.010 & 0.305 \\
$\begin{array}{l}\text { Number of graduate courses } \\
\text { College science course inquiry }\end{array}$ & 0.024 & 0.223 & -0.083 & 0.113 \\
$\begin{array}{l}\text { emphasis } \\
\text { Science ed. workshops last 5 years }\end{array}$ & 0.261 & 0.021 & -0.047 & 0.052 \\
$\begin{array}{l}\text { Conducted or assisted scientific } \\
\text { research }\end{array}$ & -0.053 & -0.058 & -0.077 & 0.024 \\
$\begin{array}{l}\text { NSES familiarity } \\
\text { KPOS familiarity }\end{array}$ & 0.031 & 0.060 & -0.047 & -0.008 \\
KY Core Content familiarity & 0.241 & 0.030 & 0.133 & 0.395 \\
\hline
\end{tabular}
$\mathrm{p}<0.10{ }^{*} \mathrm{p}<0.05$. ** $\mathrm{p}<0.01$.

When the responses of students of NBCTs were analyzed, a different pattern emerged (Table 4.19). A significant positive correlation existed for all classroom inquiry practice factors with teacher perception that their participation in science education workshops in the last five years significantly impacted their curriculum design. Teacher familiarity with Kentucky's Program of Studies and Core Content for Instruction was negatively correlated with student perceptions of classroom Use of Data $(\mathrm{p}<0.10)$ and Investigation inquiry environment factors $(\mathrm{p}<0.10)$ respectively. 
Table 4.19

Correlations of NBCT Professional Characteristics with Classroom Science Inquiry Factors

\begin{tabular}{|c|c|c|c|c|}
\hline $\begin{array}{l}\text { Pearson Correlations } \\
N=19\end{array}$ & Investigation & $\begin{array}{l}\text { Use of } \\
\text { Data }\end{array}$ & $\begin{array}{l}\text { Science } \\
\text { Dialogue }\end{array}$ & $\begin{array}{c}\text { Approach } \\
\text { to } \\
\text { Science }\end{array}$ \\
\hline $\begin{array}{l}\text { Collaboration with other science } \\
\text { teachers }\end{array}$ & 0.134 & 0.254 & 0.066 & 0.131 \\
\hline Number of graduate courses & 0.104 & 0.293 & 0.197 & 0.166 \\
\hline $\begin{array}{l}\text { College science course inquiry } \\
\text { emphasis }\end{array}$ & 0.207 & 0.136 & 0.253 & 0.225 \\
\hline Science ed. workshops last 5 years & $0.615 * *$ & $0.699 * *$ & $0.638 * *$ & $0.701 * *$ \\
\hline $\begin{array}{l}\text { Conducted or assisted scientific } \\
\text { research }\end{array}$ & -0.094 & 0.060 & -0.096 & -0.002 \\
\hline NSES familiarity & -0.244 & -0.052 & -0.313 & -0.001 \\
\hline KPOS familiarity & -0.367 & $-0.420^{\dagger}$ & -0.293 & -0.064 \\
\hline KY Core Content familiarity & $-0.401^{\dagger}$ & -0.321 & 0.240 & 0.485 \\
\hline
\end{tabular}

The distinct pattern that emerged related to the teacher characteristic Science Education Workshop Attended in the Last Five Years merited further investigation because it was significantly correlated with all four classroom inquiry environment factors. The Pearson Chi-square statistic $\chi^{2}(5)=4.422, p=0.490$, while the Likelihood ratio Chi-square statistic $\chi^{2}(5)=4.637, \mathrm{p}=0.462$, indicating that significant differences in response patterns did not exist between teacher groups defined by NBCT status. Therefore, while no significant differences existed between the frequency patterns describing NBCT and non-NBCT science education workshop attendance, NBCTs' workshop attendance was positively correlated with their students' perceptions of classroom inquiry environment, and non-NBCT workshop attendance was not. This result raises important questions about how science teachers incorporate the knowledge and skills they gain in professional development experiences into their classroom practice.

Teaching context. Students' perceptions of Science Dialogue and Approach to Science were most sensitive their non-NBCT's perceptions of the factors that determine 
their curriculum and the factors that limit inquiry. Non-NBCT perception that State or National Standards drives their curriculum was positively correlated with their students' perceptions of Investigation, Science Dialogue, and Approach to Science, while Guidance from the Principal was negatively correlated with their students' perceptions of Science Dialogue and Approach to Science. Non-NBCTs' sense that Lack of Experience limits inquiry is also significantly negatively correlated with their students' perceptions of classroom inquiry (Table 4.20).

Table 4.20

Correlations of non-NBCT Teaching Context with Classroom Science Inquiry Factors

\begin{tabular}{lcccc}
\hline Pearson Correlations & $\begin{array}{c}\text { Inves- } \\
\text { tigation }\end{array}$ & $\begin{array}{c}\text { Use of } \\
\text { Data }\end{array}$ & $\begin{array}{c}\text { Science } \\
\text { Dialogue }\end{array}$ & $\begin{array}{c}\text { Approach to } \\
\text { Science }\end{array}$ \\
\hline Factors Determining Curriculum & & & & \\
District curriculum & -0.053 & -0.002 & -0.081 & 0.052 \\
State or national standards & $\mathbf{0 . 4 3 8 *}$ & 0.218 & $\mathbf{0 . 3 6 9 *}$ & $\mathbf{0 . 3 2 7}^{\dagger}$ \\
Textbook & 0.006 & 0.053 & -0.129 & -0.066 \\
Available instructional materials & -0.145 & -0.259 & -0.087 & 0.010 \\
Guidance from principal & -0.204 & -0.123 & $\mathbf{- 0 . 3 1 7 ^ { \dagger }}$ & $\mathbf{- 0 . 3 7 6}^{*}$ \\
Other teachers' input & 0.096 & -0.260 & -0.073 & -0.233 \\
Other & -0.203 & 0.165 & -0.165 & -0.031 \\
\hline Factors Limiting Inquiry & & & & \\
Nothing limits & -0.121 & 0.126 & 0.087 & -0.112 \\
Lack of experience /awareness & -0.231 & -0.254 & $\mathbf{- 0 . 3 1 3}$ & $\mathbf{- 0 . 4 9 2 * *}$ \\
Reluctant to give up central role & -0.100 & -0.147 & $\mathbf{- 0 . 3 1 2}$ & -0.178 \\
There isn't enough time & $\mathbf{0 . 3 3 0}$ & $\mathbf{0 . 3 5 6 *}$ & 0.164 & 0.299 \\
Have tried it unsuccessfully & -0.200 & -0.074 & $\mathbf{- 0 . 4 0 4 *}$ & -0.294 \\
Doubt student inquiry capability & 0.032 & 0.144 & 0.000 & 0.091 \\
Concern about discipline, etc. & -0.033 & -0.037 & -0.186 & -0.243 \\
Insufficient time and support & 0.096 & -0.126 & -0.023 & -0.072 \\
Doesn't have materials & -0.174 & -0.239 & -0.23 & -0.019 \\
Insufficient background & -0.171 & -0.140 & -0.262 & -0.214 \\
Lack of community support & $\mathbf{- 0 . 4 0 2 *}$ & $\mathbf{- 0 . 3 6 6 *}$ & -0.216 & -0.269 \\
Lack of materials & -0.041 & -0.185 & 0.142 & 0.120 \\
Parental concerns re: text non-use & (a) & (a) & (a) & (a) \\
Disagrees with inquiry necessity & 0.064 & 0.242 & 0.063 & 0.077 \\
Other & -0.314 & -0.223 & $\mathbf{- 0 . 4 1 4}$ & -0.304 \\
\hline
\end{tabular}

(a) No variation in teacher response.

$\mathrm{p}<0.10,{ }^{*} \mathrm{p}<0.05, * * \mathrm{p}<0.01$ 
Students of NBCTs responded in different ways, however. The only teacher

response that was correlated with student perceptions was the perception of NBCTs that

Lack of Experience limits inquiry in their classrooms. This single response was

significantly negatively correlated with all four student classroom science inquiry factors

(Table 4.21).

Table 4.21

Correlations of NBCT Certified Teaching Context with Classroom Science Inquiry Factors

\begin{tabular}{lcccc}
\hline Pearson Correlations & Inves- & Use of & Science & Approach to \\
$\mathrm{N}=19$ & tigation & Data & Dialogue & Science \\
\hline
\end{tabular}

\section{Factors Determining Curriculum}

\begin{tabular}{lcccc} 
District curriculum & -0.214 & -0.327 & -0.312 & 0.038 \\
State or national standards & 0.133 & 0.118 & 0.121 & -0.081 \\
Textbook & -0.045 & 0.103 & 0.106 & 0.005 \\
Available instructional materials & 0.304 & 0.288 & 0.264 & 0.093 \\
Guidance from principal & -0.206 & -0.346 & -0.227 & -0.305 \\
Other teachers' input & 0.006 & 0.154 & 0.063 & -0.172 \\
Other & -0.305 & -0.337 & -0.259 & 0.061 \\
\hline
\end{tabular}

\begin{tabular}{lcccc}
\hline & Factors Limiting Inquiry & & \\
Nothing limits & 0.030 & -0.024 & 0.027 & 0.207 \\
Lack of experience /awareness & $\mathbf{- 0 . 5 7 3 * *}$ & $\mathbf{- 0 . 4 9 6 *}$ & $\mathbf{- 0 . 5 6 8 *}$ & $\mathbf{- 0 . 5 2 9 *}$ \\
Reluctant to give up central role & -0.020 & 0.028 & 0.030 & -0.070 \\
There isn't enough time & 0.349 & 0.324 & 0.322 & 0.147 \\
Have tried it unsuccessfully & -0.080 & -0.162 & -0.014 & -0.199 \\
Doubt student inquiry capability & 0.174 & 0.224 & 0.203 & -0.013 \\
Concern about discipline, etc. & -0.074 & 0.062 & -0.016 & -0.156 \\
Insufficient time and support & 0.111 & 0.198 & 0.192 & -0.016 \\
Insufficient background & $(\mathrm{a})$ & $(\mathrm{a})$ & $(\mathrm{a})$ & $(\mathrm{a})$ \\
Disagrees with inquiry necessity & 0.163 & 0.110 & 0.141 & -0.001 \\
Doesn't have materials & -0.090 & -0.005 & 0.057 & 0.005 \\
Lack of community support & 0.001 & 0.228 & 0.133 & 0.138 \\
Parental concerns re: text non-use & 0.001 & 0.228 & 0.133 & 0.138 \\
Other & -0.352 & -0.252 & -0.298 & $\mathbf{- 0 . 4 4 6}$ \\
\hline
\end{tabular}

(a) No variation in teacher response. $\mathrm{p}<0.10, * \mathrm{p}<0.05, * * \mathrm{p}<0.01$

This analysis indicated some relationships may have existed between teacher experiences and perceptions of scholastic environment and their students' perceptions of classroom inquiry environment. Generally, students of non-NBCTs expressed perceptions 
of classroom inquiry environment that were sensitive to their teachers' perceptions of the both the factors determining the curriculum, and the factors limiting inquiry. Additionally, a significant positive correlation existed between non-NBCTs' sense of the frequency of collaboration with other teachers and their students' perception of the investigation inquiry factor.

The perceptions of students of NBCTs, however, were much less correlated with their teacher's perceptions of factors impacting curriculum or limiting inquiry. The relative paucity of significant correlations for these teachers indicates that classrooms of NBCTs were more stable environments than those of non-NBCTs, meaning that students' perceptions of their classroom science inquiry environments did not depend on their teacher's professional experiences or perceptions of impacting curriculum. There was a significant positive correlation between the NBCTs attendance at science related workshops in the last five years and their students' perceptions of their classroom inquiry environment. Additionally, there was a significant negative correlation between NBCTs' sense that lack of awareness impacts their inquiry instruction and all four classroom inquiry environment factors describing their classrooms. This means that as one would expect, when lack of awareness of inquiry was less important to an NBCT, students reported higher levels of classroom inquiry environment.

\section{Summary}

Across the results of the four questions guiding this study both the presence of certain findings and the absence of others stands out as important. High school teachers and NBCTs displayed significantly more informed views of the role imagination and creativity play in science, and of the nature of science in general, than did middle school 
teachers or non-NBCTs. Several comparisons remained significant even under the conservative Bonferonni adjustment.

High school student perceptions of classroom inquiry environment were significantly related to the NBCT status of their teacher and the interaction of that status with their teacher's non-education degree and experience level. However, middle school student perceptions were only significantly related to the interaction between NBCT status and experience level for their teachers. The absence of observation of any middleschool teacher designed inquiry instruction confirmed this trend.

High school NBCTs appear to use data more effectively in their classrooms than any other teachers in this study. Student perception of Use of Data to Explain Results in the classrooms of high school NBCTs was significantly higher than the perception of students of any other teachers. Interestingly, Use of Data to Explain Results was also a significant predictor of science reasoning for these same students. Students of high school NBCTs also displayed significantly higher science reasoning than students of either high school non-NBCTs or students of any middle school teacher.

The perceptions of the classroom inquiry environment expressed by students of NBCTs were much less sensitive to their teacher's perception of their teaching context than were those of students of non-NBCT participants. NBCTs' perceptions of the importance of science education workshops and that lack of awareness limits inquiry were the only two factors that correlated with their students' perception of classroom inquiry environment. 


\section{DISCUSSION AND IMPLICATIONS}

\section{Discussion}

Discussion of the results is organized around the four research questions. Each section includes a summary of results, discussion and interpretation of the patterns the results display, a set of conclusions, and descriptions of the results' strength. An emerging narrative will develop through this discussion that leads to general conclusions based on convergent results from each of the research questions. The chapter closes with discussions of the implications for (a) classroom practice, (b) future research, (c) education policy, (d) teacher training, and (e) the NBPTS certification process. Question 1: How do National Board Certified science teachers' knowledge of the nature of science differ from that of their non-certified counterparts?

NBCTs displayed significantly more informed understanding of nature of science (NOS) than did their non-NBCT counterparts. By contrast, comparing teachers based on experience level or degree outside the education field showed no differential in NOS understanding. In addition to NBCTs' greater overall NOS understanding, their understanding of methodology in science was stronger than non-NBCTs. The Methodology in Science sub-scale consisted of three constructs. Understanding that: (a) Scientists do not use a single "scientific method," (b) method does not automatically produce true and accurate results, and (c) experimentation is not the only means of developing scientific knowledge (Liang, et al., 2006). Less significant differences were 
detected between NBCTs and non-NBCTs in the Imagination and Creativity in Science sub-score. The difference in this sub-score was particularly evident with high school NBCTs scoring higher than all other teachers.

Overall, teachers were most informed about Social and Cultural Influence in Science and Methodology in Science. Through all analyses however, (and for both teacher and students), the sub-scales corresponding to Scientific Laws vs. Theories, and Imagination and Creativity in Scientific Investigations continually displayed markedly lower scores than other sub-scales and were mostly negative, indicating uninformed views. Examination of the descriptive statistics for these sub-scales indicated that teachers' understanding of the Scientific Laws vs. Theories section was generally much lower than Imagination and Creativity in Scientific Investigations. Analysis of the individual questions on the assessment indicated that interpretation of the Scientific Laws $v$ s. Theories questions hinged solely on understanding the differences in the definitions of "theory" and "law" as applied to scientific knowledge. In the case of the Imagination and Creativity questions, the use of the key terms was much more commonplace. The discrepancies in results for these two sections of the test may have resulted because the true definitions of "imagination" and "creativity" were much more aligned with colloquial usage than were the definitions of "theory" and "law."

This common trend of NOS understanding among both teachers and students raises the question of whether teachers and students shared the same misconceptions because they shared the experience of the same broader culture which was providing the only definitions of these terms, or if students possessed these misconceptions because they were taught them by their teachers. Given the paucity of explicit NOS instruction in 
U.S. schools (Abd-El-Khalick, Bell, \& Lederman, 1998), the former explanation is more likely.

Because these were simple comparisons of scores between groups, these differences in NOS understanding may have arisen due to factors other than NBCT status. For example, a threat of history to the validity of these findings is present because the professional, educational and professional development experiences of participating teachers were not collected. This validity threat was addressed to some extent when experience level, pedagogical training as operationalized by certification rank, and noneducation degree were all eliminated as potential counterfactuals. The lack of relationship of these last three variables with teacher NOS understanding supports earlier research on the topic (Abd-El-Khalick \& Lederman, 2000). Abd-El-Khalick and Lederman (2000) found that implicit instruction, defined as providing experiences that involved participants in the processes of science without calling their attention to NOS concepts, did little to change the NOS conceptions of the teachers involved. Furthermore, explicit instruction, defined as providing experiences involving participants in the processes of science and providing opportunity for discussion and reflection on the meaning of those experiences, seemed to provide more consistently effective results.

A variety of interpretations are consistent with the data. In light of the lack of difference in NOS understanding associated with non-education degree and the lack of emphasis on NOS understanding in the NBPTS certification process, attention should be paid to other profession development experiences as the source of this knowledge differential. Perhaps the National Board Certification process sensitizes candidates to qualities of professional development experiences that effectively impact their practice. 
Earlier studies have identified qualities of NBCTs that may shed light on the NOS understanding differences observed in this study. Among other teacher qualities, NBCTs showed greater expertise in the areas of (a) use of knowledge, (b) pedagogical content knowledge, (c) problem solving strategies, (d) creating classroom climate, (e) testing of hypotheses, and (l) passion for teaching (Bond, Smith, Baker, \& Hattie, 2000). Possessing greater capacity in these areas may well provide a broader foundation from which NBCTs are able to display differentially greater NOS understanding than nonNBCTs.

A different hypothesis is that the certification process increases NBCT selfefficacy and personal agency, so that they feel greater capacity or responsibility to transform their practice in response to new knowledge and experiences. Self efficacy has been related to levels of teacher planning and organization (Allinder, 1994), and willingness to try new methods to meet the needs of students (Berman, McLaughlin, Pauly, \& Zellman, 1977). Additionally, greater self efficacy was positively related to teachers' enthusiasm for teaching (Allinder, 1994), and commitment to teaching (Coladarci, 1992). If middle and high school science NBCTs have greater self-efficacy, their increased enthusiasm and commitment to teaching may easily increase their interest in all areas of science including NOS concepts.

Evidence from teacher interview in this study also indicates that NBCTs may have greater personal agency than other teachers. Personal agency concerns how people exercise control over their lives (Goddard, Hoy, \& Woolfolk-Hoy, 2004). It extends self efficacy to include both self-efficacy beliefs and context beliefs which are evaluations of the responsiveness of one's environment (Ford, 1992). Self efficacy and personal agency 
beliefs have been shown to have important relationships to teaching practice. Haney, Lumpe, Czerniak, and Egan (2002) found that teachers with high personal agency beliefs were more likely to design instruction incorporating inquiry, attend to student prior knowledge, and use available resources. These teachers are also more likely to present science content that was appropriate for their students in an interesting and engaging way. Teachers who are engaged with their professional practice in this way may be more likely to conscientiously attend to the deeper patterns in science constituting nature of science concepts.

These studies just described indicate that there may be interesting relationships between NBCT self-efficacy and personal agency, and their higher levels of NOS understanding. Studies describing NBCT self-efficacy and personal agency may uncover reasons why NBCTs have been shown to be more effective at providing instruction. They may also begin to explain why the NBCTs in this study displayed greater NOS understanding in spite of their apparent similarity with other teachers on important professional characteristics. Studies investigating the capacity among NBCTs to learn NOS, and subsequently to design and deliver effective NOS instruction, may provide useful information for teacher preparation programs and professional development providers to enhance NOS professional development for all teachers.

Question 2: How do the frequencies of student science inquiry behaviors supported by the learning environments created by NBCTs differ from those created by their nonNBCT counterparts?

Students provided more reliable measures of middle and high school science classroom inquiry learning environments than teachers regardless of the extent of their 
teacher's experience, or NBCT status. The factors in the measurement of science classroom inquiry learning environment also tended to collapse into factors that mirrored those of the teachers who participated in the initial validation of the instrument (Dunbar, 2002). This lent credence to the original structure of the instrument and its applicability in this study. This result also confirms earlier research with younger students (Teddlie \& Stringfield, 1993). A possible explanation for this is science teacher perceptions of their classrooms were confounded by professional training, perceptions of professional working environment, hopes and intentions, and the distractions of actually teaching.

Primary analysis was disaggregated by middle/high school assignment because of potentially important differences between the two groups. Middle and high school science teachers tend to differ considerably on their content and pedagogical preparation, and instructional climate of middle and high schools also tend to differ in important ways. The classrooms of high school NBCTs were characterized by significantly more frequent science inquiry related behaviors than those of both middle and high school nonNBCTs. Additionally, the interaction of teacher NBCT status with experience and noneducation degree seemed to augment the benefit of high school NBCT status. This was evident because neither experience nor non-education degree were significant predictors of classroom inquiry environment by themselves, while their interactions with NBCT status were. This points to potentially interesting relationships suggesting NBCT status for high school science teachers may enable other factors to have significant impacts.

Middle school science classrooms presented a different pattern. There was no main effect of NBCT status, experience level, or non-education degree on frequency of science inquiry behaviors. However, interactions between middle school NBCT status 
with experience and non-education degree were both significantly related to classroom science inquiry environment. In particular, to a greater extent than with high school teachers, the interaction with NBCT status and experience seemed to be influential for middle school teachers.

Overall, these results are important because the nature of the interaction of National Board Certification with other teacher qualities has not been previously established. A potential explanation for the patterns emerging from this study is that National Board Certification status provides recipients a sense of personal agency with which they can better apply the practical and theoretical knowledge imbued by longer experience and higher levels of education. Personal agency has been defined as the belief in one's capability to regulate one's own behavior to attain goals (Zimmerman \& Cleary, 2006). A plausible hypothesis is that NBCTs might have greater personal agency because the NBPTS certification process is intensely analytic and reflective, and successful certification rests on being able to provide trustworthy evidence demonstrating the impact of instruction on student achievement. Cuban (1995) described schools as coping organizations because they do not have consensus on their desired product, cannot directly observe the process of production, and have difficulty describing how they make their products. Within a challenging environment such as this, differences in teachers' sense of personal agency might easily be reflected in their classrooms.

Interestingly, only the classroom technique Use of Audio-Visual Materials showed difference between groups defined by NBCT status, and Use of Other Materials was different between groups defined by experience level. None of the teacher general pedagogical practice variables describing teacher practice, such as amount of homework 
assigned each week or number of minutes spent in lab, was related to student perceptions of classroom inquiry environment. Ambiguity in the prompts or scales describing these behaviors may have limited the power of the instrument to detect differences in teacher practice. The lack of correlation between general pedagogical practice variables and science inquiry learning environments may also have originated from the same sort of confounding of teacher perception that affected participants' responses to the classroom inquiry environment instrument.

Field observations of a stratified random sample of participant teachers positively triangulated results from student perceptions of the classroom inquiry environment. In agreement with what was found for classroom science inquiry environment based on student responses, the field observations showed that high school science teachers were able to plan and enact a lesson the focus of which was inquiry or that used inquiry as the mode of instruction for science content. The high school NBCTs were able to select and enact lessons that engaged their entire class with inquiry behaviors that fit within a longterm plan of instructional goals. Although, the non-NBCT high school teacher was able to select and enact an inquiry lesson, it was less well implemented, with less purposeful use of equipment and materials to support the instruction and student learning.

Almost none of the middle school teachers selected an inquiry lesson for observation even though this was explicitly requested. A single middle school teacher did produce an inquiry lesson, but she was not at liberty to make a choice because her district dictated her curriculum. Additionally, her reason for liking the district mandated FOSS modules promoting inquiry instruction was that because they were kits provided by the district, she did not have to buy materials with her own money—not because they 
provided a particularly good inquiry curriculum. The single middle school NBCT observed recognized that his lesson was a poor example of inquiry, but in the severalweek time frame in which to select a lesson that lesson was the best he could produce. This indicates that possibly he did not use inquiry regularly in his science classroom. Additionally, one middle school teacher expressed her perspective that inquiry was not an appropriate instructional mode for middle school students learning science.

The field observations tended to confirm the differences in practice and knowledge uncovered in the NOS and classroom inquiry environment instrumentsNBCTs used more inquiry than non-NBCTs and high school teachers used more inquiry than middle school teachers. Although these were single observations and, therefore, were less reliable measures of teacher practice than observations of multiple class periods, confidence in this interpretation is enhanced because these qualitative results triangulated with the quantitative results from the larger number of teachers.

The evidence that no middle school science teachers responded with inquiry lessons to the request for observation of an inquiry-related lesson raises a question of whether this circumstance was idiosyncratic. That is, was it related only to the lesson on the date of the observation, or was it systematic, meaning lack of inquiry instructional focus was a general characteristic of these middle school teachers? The agreement of results from measurement of classroom inquiry environment data from field observations provides evidence for the merit of the second hypothesis.

Question 3: What is the relationship between the frequency of students' science inquiry behaviors and their science reasoning abilities and understanding of the nature of science? 
Student science reasoning. Student science reasoning was positively correlated with the Use of Data to Explain inquiry factor in both NBCT and non-NBCT high school classrooms. This pattern emerged when analysis was disaggregated according to the fourlevel variable defined by NBCT status and school level assignment. This disaggregation was chosen for exploration because patterns were seen in teacher and student data that indicated this interaction might be of some interest. The Use of Data to Explain factor predicted student science inquiry score only for high school students but not middle school students. This result may relate primarily to the nature of data use in high schools rather than its frequency. This hypothesis is based on the result that although the Use of Data to Explain factor was rated as occurring significantly more frequently in high school NBCT classrooms than high school non-NBCT classrooms, the relationship was significant for students of both NBCTs and non-NBCTs. The difference in significance levels ( $\mathrm{p}<0.05$ for high school NBCTs and $\mathrm{p}<0.10$ for high school non-NBCTs) may indicate an indirect effect of NBCT status on student science reasoning mediated through the classroom use of data to explain observations.

The Use of Data to Explain factor consists of five questions prompting the participant to rate the frequency of the following behaviors on a scale anchored by "never" and "almost exclusively."

1. Memorize scientific facts or information independently (negatively coded).

2. Use data to construct reasonable explanation.

3. Seek and recognize patterns (trends in the data or observations).

4. Detect sequences (the order of events in a science activity) (Dunbar, 2002). 
5. Follow a set series of steps to get to the right answer to a question (negatively coded).

These five inquiry-oriented activities have face-validity regarding their capacity to impact science reasoning skill. Additionally, Lawson and Bealer (1984) indicated that the LTSR was sensitive to the effects of instruction. Describing the frequency of science inquiry activities alone however, provides only limited information regarding the role these activities play in improving student science reasoning. These activities may be enacted in a variety of ways leading to greater or lesser change in student inquiry abilities. In this study the NBCTs may have enacted these activities more effectively for their students, resulting in the associated difference in student science reasoning skills. If future studies substantiate this hypothesis, this result would provide guidance to curriculum developers and to teachers describing which classroom practices may be most effective for enhancing student science reasoning skills.

The classroom inquiry environment Approach to Science factor was a significant negative predictor of student science reasoning for middle school students of nonNBCTs. This factor consisted of responses to four ESIS questions:

1. Use drawing, graphing, or charting to convey new information from a science activity?

2. Show reluctance to ask questions that might extend an activity? (negatively coded)

3. Assess their own or their group's work?

4. Use computers or calculators while conducting investigations? (Dunbar, 2002) 
The observed relationship means middle school students of non-NBCTs had science reasoning scores that were lower when these four inquiry behaviors were more frequent. One might think this puzzling relationship may have originated in differential grade-level assignment between middle school NBCT and non-NBCT participants. Non-NBCTs taught sixth, seventh, and eighth grade students, while NBCTs taught only seventh and eighth graders. This potential counterfactual was found to be irrelevant, however, because student grade level was not a significant predictor of science reasoning for students of any group of middle school teachers. The confluence of this result distinguishing classrooms of middle school science non-NBCTs with the significantly lower NOS understanding of their students shown in other analyses raises questions about the activities occurring in those classes. Are there identifiable practices in those classes that contribute to both decreased science reasoning and limited NOS understanding?

Student NOS understanding. Student NOS understanding was not sensitive to any classroom inquiry environment operationalized in this study. This supports earlier research suggesting that implicit NOS instruction is relatively unsuccessful (Abd-ElKhalick \& Lederman, 2000). In an earlier study, Abd-El-Khalick (1998) found that in spite of calls by the National Science Education Standards (NSES) (NRC, 1994), U.S. science classrooms remain mostly devoid of explicit NOS instruction. Furthermore, in a review of biology textbooks, Lumpe and Beck (1996) found that science facts were still the main focus of the texts, and that several major strands of NOS understanding were not integrated into the text. The disconnect present in this study between classroom inquiry environment and NOS understanding may indicate that none of the teachers participating in this study provided effective explicit NOS instruction. 
Differences were noted on first phase of assessment. Differences in science reasoning and NOS understanding between students' of NBCTs and non-NBCTs existed in October during the first phase of assessments. No significant changes in students' scores occurred between the first and second phases of assessment for students' of any group of teachers. This initial difference between students of NBCTs and non-NBCTs and lack of subsequent growth for either group potentially can be explained in several ways. A possible hypothesis is that the students of NBCTs began their school year with higher levels of science reasoning than students of non-NBCTs and that neither group of science teachers produced any change in student science reasoning ability. A different, but functionally equivalent state of student learning is that students started the year with a lower level of science reasoning ability that was already differentiated between NBCT and non-NBCT groups. In this model, during the first part of the year both groups of students did indeed learn science reasoning skills from their teachers, no differential change was created by instruction. By the time they were measured in phase I of this study, they had stopped developing new skills and remained constant for the remainder of the semester. These potential counterfactuals were addressed by examining the student demographic variables. No demographic differences were found between students of NBCTs and their non-NBCT counterparts, however, diminishing the potential likelihood of these counterfactuals.

A more likely situation—one that this study was not designed to address—was that the primary instruction affecting science reasoning occurred early in the year and had become a status variable by the date of the first phase of assessments. This hypothesis is viable because many science curricula provide primary instruction in science inquiry 
skills including control of variables, observation and inference, and experimental design at the beginning of the year. Understanding the viability of this hypothesis to explain potential changes in student science reasoning skills would require data collection from the beginning of the course rather than two months after its start as was the case in this study.

Question 4: What is the impact of teacher perceptions factors impacting curriculum and limiting inquiry on the existence of inquiry learning environments?

Student perceptions of classroom environment were correlated with teacher NBCT status. None of the factors determining curriculum was correlated with student perceptions of classroom inquiry environment. NBCT classrooms were less sensitive to teachers' prior experience or their perceptions of factors impacting curriculum and inquiry practice than the classrooms of non-NBCT participants. The apparent ability of NBCTs to control their classroom environment was corroborated by observation and interview during which both high school NBCTs volunteered their perceptions of the control they were able to exert in their classroom, limiting the impact school environmental factors had on their classroom environment. The only teacher perception correlated with student perceptions of classroom environment was Lack of Experience and Awareness of Inquiry. This factor was negatively correlated with all four classroom inquiry environment factors. The NBCTs' perception had a stronger relationship to student perceptions of classroom inquiry environment than any other teacher perception for either NBCTs or non-NBCTs.

Perceptions of classroom inquiry environment expressed by students of nonNBCTs on the other hand, were sensitive to their teachers' perceptions of (a) factors 
influencing curriculum, (b) factors limiting inquiry, and (c) frequency of collaboration with other teachers. Several teacher perceptions of factors impacting curriculum and factors limiting inquiry were correlated with their classroom inquiry environment. A possible explanation is that non-NBCTs were less able to create a controlled intentional learning environment in their classrooms, and factors other than their pedagogical intentions played important roles affecting their classroom environments.

This differential sensitivity of classroom inquiry environment to school environment and teacher inquiry efficacy perceptions may have originated in differential sense of personal agency among teachers. Haney et al. (2002, p. 172) describe personal agency as "evaluative beliefs comparing a person's goals with the consequences of their pursuit of those goals." Ford (1992) describes the importance of personal agency as being of greatest importance to challenging but attainable goals. The National Board Certification process may have made NBCTs aware of the importance of the inquiry learning occurring in their classrooms. The National Board Certification process may have also affirmed their capacity to create a learning environment in which their students could achieve at high levels. The learning environments the NBCTs created consequently may have been focused on their instructional goals and less dependent on environmental factors present in the school at large. Furthermore, experiences NBCTs gathered during professional development experiences seemed to be more consistently applied to their instruction, positively affecting their students' perceptions of their classroom inquiry environment.

An important result is that NBCTs' report of science workshop attendance in the last five years was significantly correlated with student perceptions of inquiry learning 
environment. However, the data suggested that non-NBCTs attended science workshops at about the same frequency as NBCTs, and yet non-NBCT students' perceptions of inquiry learning did not correlate with workshop attendance. The existence of this relationship for NBCTs but not others implies that NBCTs were more effectively able to convert those workshop experiences into science inquiry classroom climate more for their students.

An important limitation to the interpretation of these results is that these were teacher perceptions of factors influencing curriculum and limiting inquiry, not direct measures of those constructs. Teacher perception that a particular factor is important could mean either that it actually plays an important role, or that the teacher is particularly sensitive to the effect of that factor. The explicit focus on science inquiry in the NBPTS certification process may have made these NBCTs more critically aware of their level of competence, thus increasing the validity of their self-evaluation.

\section{Implications}

National Board Certification provides professional-level identity for teachers (NBPTS, 2006a). This study provides evidence that this certification process may significantly impact teacher practices and student knowledge. Having strived to obtain this level of certification, NBCTs conception of themselves as teachers may be different than that of other teachers. NBCTs may be more likely to purposefully consider what curriculum is important and how it should best be delivered.

Classrooms are situated within institutions possessing strong cultures affecting the experiences of all those who practice or learn in them (Cuban, 1995). Middle and high school students' lives encompass experiences, relationships and histories outside the 
control of their teacher but impacting learning nonetheless. For these reasons, regardless of the effectiveness of the teacher, only a limited relationship may exist between any specific teacher or student practice and particular student effects.

\section{Implications for Classroom Practice}

Science reasoning and NOS understanding were narrowly construed by the methodology and instrumentation used in this study. Both topics may be better evaluated with performance assessment and interview. This said, this study did produce results that may inform middle and high school science teacher practice. This study confirms that using data to explain observations and inferences positively affects students' science reasoning capacity. This study also confirms that implicit instruction covering nature of science concepts does not effectively change students' NOS understanding.

Perhaps as interesting as the relationship between classroom use of data and student science reasoning is the absence of such a relationship for the other classroom inquiry environment factors. Student behaviors such as asking questions, using science process skills, performing self-motivated experiments, and defending the adequacy or logic of statements were not related with student science reasoning. This lack of relationship may exist because the behaviors were not enacted with sufficient frequency in these classrooms, not enacted with sufficient effectiveness, or that they had no close relationship with science reasoning as it was operationalized on the science reasoning assessment. This study indicates that investigations into the relationship among these behaviors and student science reasoning may lead to important results informing inquiry teaching practice. 
Prior research has shown that teachers share many of the NOS misconceptions of their students (Anderson, Sheldon, \& Dubay, 1990; Sanders, 1993) and that curriculum materials are not particularly supportive of explicit NOS instruction (Hipkins, Barker, \& Bolstad, 2005). Special care should be taken by teachers to become more informed regarding NOS and to construct their own lessons explicitly addressing NOS-not relying on their texts to organize the material for them. This may include attending NOS oriented professional development or engaging in graduate-level coursework.

\section{Implications for Future Research}

Future research that focuses on the entire arc of instruction might shed brighter light on relationships between science teacher characteristics and teacher practice. Multiple classroom observations of the observed teachers would increase the validity of inferences drawn about typical instruction. Including interviews of teachers that reveal the process they follow during curriculum design and instructional planning may enhance inferences about what priorities affect teacher decisions at various points during the planning-execution cycle.

This study raises many questions of interest. Differences were detected between NBCTs and their non-NBCT counterparts' understanding of NOS. Is this difference simply correlated with NBCT status, or is it related in some way to the experience of attempting National Board Certification? What is the relationship between the National Board Certification process and teachers' sense of self-efficacy and personal agency? What are the nature and frequency of Use of Data to Explain activities that are related to student science understanding? Why did differences in teacher practice exist between teachers according to middle/high school assignment? 
More broadly, why is NOS understanding constructed as it is, with some areas being relatively strong and others relatively weak? Why do teachers and their students share the same areas of weakness in their NOS understanding? And perhaps most important, what experiences and resources would best enable teachers to construct units providing effective NOS instruction?

\section{Implications for Policy}

Several states have instituted policies supporting teachers as they attempt National Board certification, providing incentives for them to attain NBPTS certification, or both (NBPTS, 2007). This policy trend rests on the assumption that NBCTs are valuable professionals in classrooms. This study tends to confirm that NBCT status is an indicator of higher science teacher quality. If state governments view the certification process itself as inherently beneficial, as appears to be the case, evidence of its efficacy improving teacher quality should be established. This study does not provide evidence to inform these decisions because National Board Certification was a status variable for teachers in this study. This means that teachers' NBCT status was fixed before the beginning of the study and did not change during the study, therefore, the effect of pursuing National Board Certification on teachers' science inquiry instruction skills could not be assessed by this study.

This study supports the use of teacher incentives for NBCT status as a form of merit pay, however, as is a topic of discussion in Kentucky. NBCTs in this study were distinguished by their capacity to positively influence classroom inquiry environment and, in certain cases, to positively influence measures of student science reasoning. 
Furthermore, NBCTs seem to have a greater capacity to transform professional development experiences into positive classroom inquiry environments for their students. Implications for Teacher Education

Student NOS understanding, science inquiry, and science reasoning are explicit components of state and national standards (NRC, 1994, AAAS, 1993). Many of the study's participating teachers were poorly informed regarding NOS concepts. If, as appears to be the case, explicit instruction is the most effective method to change understanding and skills in these domains, then explicit attention on these understandings and skills should be a focus of middle and secondary science teacher preparation programs. Additionally, research should focus on the development of effective methods by which teachers can incorporate explicit NOS instruction into the content instruction mandated by states and administrations.

\section{Implications for the NBPTS Certification Process}

Both the NBPTS Early Adolescent (middle school) and Adolescent/Young Adult (high school) Science Standards refer explicitly to scientific habits of mind and the processes of active science inquiry as important subjects for teachers to know and teach to their students (NBPTS, 2003a, 2003b). These descriptions follow the NSES (NRC, 1994) closely. They do not, however, describe NOS concepts in the way conceptualized by Lederman (1992) and others. Earlier work by the author indicated that NBPTS Adolescent/Young Adult Science candidates attended very carefully to portfolio instructions and rubrics as they planned and constructed their responses (Saderholm, 2005). From this perspective a reasonable hypothesis is that if any cognitive changes due to the certification process had occurred in the NBCTs participating in this study, those 
changes would not be consonant with the conception of NOS espoused by Lederman (1992) and others, and upon which the SUSSI instrument was developed. Therefore, while NBCTs may possess understanding of scientific habits of mind and dispositions present in the NSES, they may still lack understanding of the nature of science. This lack of understanding may prevent NBCTs from being any better at providing explicit NOS instruction than other teachers.

Differences were detected between middle school and high school science NBCTs. High school science NBCTs were associated with classroom science inquiry environments that promoted the science reasoning skills of their students, however, no such relationship was detected for middle school science NBCTs. Further research is indicated to verify if this result is generalizable to the population of high school and middle school science NBCTs. If research indicates that these results are generalizable, further research may be warranted into what confluence of educational, experiential, and environmental factors contribute to the differences and what changes are suggested to ameliorate them.

\section{Conclusion}

This study was designed to identify differences between teachers regarding important science inquiry environmental factors and the consequent effect on their students reasoning and understanding. High school NBCTs implemented inquiry practice more frequently their non-NBCT counterparts, but no differences were detected between middle school NBCTs and non-NBCTs. NBCTs were better able to profit from experience and education than non-NBCTs. Furthermore, differences were detected that suggested that NBCTs were better able to create intentional learning environments, 
insulated their classrooms from outside influences. Due to limitations inherent in this design however, these differences should not be construed to be definitive but instead suggest directions for larger scale studies or studies using methodology designed to delve more deeply into the interactions among the various constructs.

Furthermore, unexpected differences between middle and high school teachers, the sensitivity of high school students to data use in their classroom while middle school students showed no such sensitivity, and the interesting emerging themes relating National Board Certification to teacher efficacy and personal agency provide many avenues for continued research.

At the high school level, the relationship between using data to explain results and science reasoning skills merits further investigation. Does this represent a particularly useful pedagogical strategy? Or was this result the consequence of how using data and science reasoning were operationalized in this study? Further, why wasn't this relationship evident at the middle school level?

The primary goal of this study was to discern if NBCTs had greater capacity to create science inquiry learning environments. Another important goal was to identify teacher behaviors and classroom environment factors that would lead to improved student learning. For these reasons, this study may be characterized as successful. It identified important differences between NBCTs and non-NBCTs and between middle and high school teachers, and highlighted interesting interactions for future research. 


\section{REFERENCES}

Abdal-Haqq, I. (1996). Making time for teacher professional development. (Report No. EDO-SP-95-4). Eric Digest. Washington, DC: Office of Educational Research and Development. (ERIC Document Reproduction Service No. ED400259)

Abd-El-Khalick, F., Bell, R., \& Lederman, N. (1998). The nature of science and instructional practice: Making the unnatural natural. Science Education, 82(4), 417-436.

Abd-El-Khalick, F., \& Lederman, N. (2000). Improving science teachers' conceptions of nature of science: A critical review of the literature. International Journal of Science Education, 22(7), 665-701.

Allinder, R.M. (1994). The relationship between efficacy and the instructional practices of special education teachers and consultants. Teacher Education and Special Education, 17(2), 86-95.

American Association for the Advancement of Science. (1990). Science for all Americans New York: Oxford University Press.

American Association for the Advancement of Science. (1993). Benchmarks for science literacy. New York: Oxford University Press.

Anderson, C.W., Sheldon, T.H., \& Dubay, J. (1990). The effects of instruction on college nonmajors' conceptions of respiration and photosynthesis. Journal of Research in Science Teaching, 27(8), 761-776.

Anderson, R.D. (2002). Reforming science teaching: What research says about inquiry. Journal of Science Teacher Education, 13(1), 1-12.

Appeldoorn, K.L. (2004). Developing and validating the Collaboratives for Excellence in Teacher Preparation (CETP) Core Evaluation Classroom Observation Protocol $(C O P)$. Unpublished Dissertation, University of Minnesota.

Armor, D., Conroy-Oseguera, P., Cox, M., King, N., McDonnell, L., Pascal, A., et al. (1976). Analysis of the school preferred reading program in selected Los Angeles minority schools (No. R-2007-LAUSD). Santa Monica, CA: Rand, Corp. (ERIC Document Reproduction Service No. ED130243) 
Baker, W.P., Lang, M., \& Lawson, A.E. (2002). Classroom management for successful student inquiry. The Clearing House, 75(5), 248-252.

Bandura, A. (1977). Self-efficacy: Toward a unifying theory of behavioral change. Psychological Review, 84(2), 191-215.

Bandura, A. (2006). Toward a psychology of human agency. Perspectives on Psychological Science, 1(2), 164-180.

Berman, P., McLaughlin, M., Bass, G., Pauly, E., \& Zellman, G. (1977). Federal programs supporting educational change, vol. VII: Factors affecting implementation and continuation (No. R-1589/7-HEW). Santa Monica, CA: Rand Corp.

Blumenfeld, P., Fishman, B.J., \& Krajcik, J. (2000). Creating usable innovations in systemic reform: Scaling up technology-embedded project-based science in urban schools. Educational Psychologist, 35(3), 149-164.

Bond, L., Smith, T., Baker, W., \& Hattie, J. (2000). The certification system of the National Board for Professional Teaching Standards: A construct and consequential validity study: National Board for Professional Teaching Standards.

Bredo, E. (2000). Reconsidering social constructivism: The relevance of George Herbert Meade's interactionism. In D. C. Philips (Ed.), Constructivism in education: Opinions and second opinions on controversial topics (pp. 127-158). Chicago: The University of Chicago Press.

Brown, S. L., \& Melear, C.T. (2006). Investigation of secondary science teachers' beliefs and practices after authentic inquiry-based experiences. Journal of Research in Science Teaching, 43(9), 938-962.

Bull, B., Beuchler, M., Didley, S., \& Krehbeil, L. (1994). Professional development and teacher time: Principles, guidelines, and policy options for Indiana. Indianapolis, IN: Indiana State Department of Education.

Burry-Stock, J.A., \& Oxford, R.L. (1993). Expert science teaching educational evaluation model (esteem) for measuring excellence in science teaching for professional development. Washington, DC: Office of Educational Research and Improvement.

Byrne, B. (1998). Structural equation modeling with Lisrel, Prelis, and Simplis: Basic concepts, applications, and programming. Mahwah, NJ: Lawrence Erlbaum Associates, Publishers.

Calabrese Barton, A., \& Yang, K. (2000). The culture of power and science education: Learning from Miguel. Journal of Research in Science Teaching, 37(8), 871-889. 
Carnegie Forum on Education and the Economy (1986). A nation prepared: Teachers for the 21 st century. The report of the task force on teaching as a profession. Washington, D.C.: The Task Force on Teaching as a Profession. (ERIC Document Reproduction Service No. ED 268 120)

Cannon, J. R., \& Scharmann, L.C. (1996). Influence of a cooperative early field experience on preservice elementary teachers' science self-efficacy. Science Education, 80(4), 419-436.

Cavalluzzo, L.C. (2004). Is National Board Certification an effective signal of teacher quality? The CNA Corporation.

Chaney, B. (1995). Student outcomes and the professional preparation of eighth-grade teachers in science and mathematics. NSF/NELS:88 teacher transcript analysis. National Science Foundation, Arlington, VA.

Chang, C., \& Mao, S. (1999). Comparisons of Taiwan science students' outcomes with inquiry-group versus traditional instruction. Journal of Educational Research, 92(6), 340-346.

Cohen, J. (1988). Statistical power analysis for the behavioral sciences (2 ed.). Hillsdale, NJ: Lawrence Erlbaum Associates, Publishers.

Coladarci, T. (1992). Teachers' sense of efficacy and commitment to teaching. Journal of Experimental Education, 60(4), 323-337.

Crawford, B.A., Krajcik, J.S., \& Marx, R.W. (1999). Elements of a community of learners in a middle school science classroom. Science Education, 83(6), 701-723.

Colburn, A. (2000). An inquiry primer. Science Scope, 23(6), 42-44.

Cook, T.D., \& Campbell, D.T. (1979). Quasi-experimentation: Design \& analysis issues for field settings. Boston: Houghton Mifflin.

Cromer, A. (1993). Uncommon sense: The heretical nature of science. Oxford: Oxford University Press.

Cuban, L. (1995). The hidden variable: How organizations influence teacher responses to secondary science curriculum reform. Theory into Practice, 34(1), 4-11.

Damnjanovic, A. (1999). Attitudes toward inquiry-based teaching: Differences between preservice and in-service teachers. School Science and Mathematics, 99(2), 71-76.

Darling-Hammond, L. (1999). America's future: Educating teachers. Education Digest, 64(9), 18-23.

DeBoer, G.E. (1991). A history of ideas in science education: Implications for practice. New York, NY: Teachers College Press. 
Denzin, N.K. (1994). The art and politics of interpretation. In N.K. Denzin \& Y.S. Lincoln, (Eds.), Handbook of qualitative research. Thousand Oaks, CA: Sage.

Desautels, J. (1998). Construction-in-action: Students examine their idea of science. In M. Larochelle, Bednarz, N., and Garrison, J. (Eds.), Constructivism in education (pp. 121-138). Cambridge: Cambridge University Press.

Dewey, J. (1910). Science as subject-matter and as method. Science, 31(787), 121-127.

Dewey, J. (1933). How we think: A restatement of the relation of reflective thinking to the educative process. Boston: D.C. Heath and Company.

Diamond, J. (1999). Guns, germs, and steel: The fates of human societies. New York, NY: W.W.Norton \& Company, Inc.

Dreyfus, H.L., Dreyfus, S.E., with Athanasiou, T. (1986). Mind over machine : The power of human intuition and expertise in the era of the computer New York: Free Press.

Dunbar, T.F. (2002). Development and use of an instrument to measure scientific inquiry and related factors. Unpublished Dissertation, The University of New Mexico, Albuquerque, NM.

Duveen, G. (1997). Psychological development as a social process. In L. Smith, Dockrell, J., and Tomlinson, P. (Ed.), Piaget, Vygotsky and beyond: Future issues for developmental psychology and educations. New York: Routledge.

Enochs, L. G., Scharmann, L.C., \& Riggs, I.M. (1995). The relationship of pupil control to preservice elementary science teacher self-efficacy and outcome expectancy. Science Education, 79(1), 63-75.

Ercikan, K., \& Roth, W-M. (2006). What good is polarizing research into qualitative and quantitative? Educational Researcher, 35(5), 14-23.

Fenstermacher, G.D., \& Richardson, V. (2005). On making determinations of quality in teaching. Teachers College Record, 107(1), 186-212.

Ferguson, E. (2003). Closing the achievement gap: Two views from current research. ERIC digest. Washington, DC: ERIC Clearinghouse on Urban Education. (ERIC Document Reproduction Service No. ED482919)

Flick, L.B. (1995). Complex instruction in complex classrooms: A synthesis of research on inquiry teaching methods and explicit teaching strategies. Paper presented at the annual meeting of the National Association for Research in Science Teaching in San Francisco, CA., April 22-25, 1995.

Ford, M.E. (1992). Motivating humans : Goals, emotions, and personal agency beliefs. Newbury Park, CA: Sage Publications. 
Geertz, C. (1973). The interpretation of cultures: Selected essays. New York: Basic Books.

Gibson, H.L., \& Chase, C. (2002). Longitudinal impact of an inquiry-based science program on middle school students' attitudes toward science. Science Education, 86(5), 693-705.

Giovannelli, M. (2003). Relationship between reflective disposition toward teaching and effective teaching. The Journal of Educational Research, 96(5), 293-309.

Glaser, B.G., \& Strauss, A.L. (1967). The discovery of grounded theory: Strategies fo qualitative research. Chicago: Aldine.

Goddard, R.D., Hoy, W.K., \& Woolfolk-Hoy, A. (2004). Collective efficacy beliefs: Theoreticai developments, empirical evidence, and future directions. Educational Researcher, 33(3), 3-13.

Goldhaber, D., \& Anthony, E. (2005). Can teacher quality be effectively assessed? National Board Certification as a signal of effective teaching: The Urban Institute. Retrieved on June 11, 2006 from http://www.urban.org/UploadedPDF/411271_teacher_quality.pdf.

Goldhaber, D., \& Brewer, D. (1998). When should we reward degrees for teachers? Phi Delta Kappan, 80(2), 134, 136-138.

Goldhaber, D., \& Brewer, D. (1996). Evaluating the effect of teacher degree level on educational performance. Rockville, MD: Westat, Inc. (ERIC Document Reproduction Service No. ED406400)

Griffard, P.B., \& Wandersee, J.H. (1999). Challenges to meaningful learning in AfricanAmerican females at an urban science high school. International Journal of Science Education, 21(6), 611-632.

Hammond, L. (2001). Notes from California: An anthropological approach to urban science education for language minority families. Journal of Research in Science Teaching, 38(9), 983-999.

Haney, J.J., Lumpe, A.T., \& Czerniak, C.M. (2002). From beliefs to actions: The beliefs and actions of teachers implementing change. Journal of Science Teacher Education, 13(3), 171-187.

Haury, D.L. (1993). Teaching science through inquiry. ERIC/CSMEE digest. (No. EDOSE934). Washington, DC.: Office of Educational Research and Improvement (ERIC Document Reproduction Service No. 359048)

Hausfather, S.J. (1996). Vygotsky and schooling: Creating a social context for learning. Action in Teacher Education, 18(2), 1-10. 
Hein, G.E., \& Lee, S. (2000). Assessment of science inquiry. In Foundations: A monograph for professionals in science, mathematics, and technology education (pp. 99-108). Washington, DC: National Science Foundation.

Hipkins, R., Barker, M., \& Bolstad, R. (2005). Teaching the "nature of science": Modest adaptations or radical reconceptions? International Journal of Science Education, 27(2), 243-254.

Hofstein, A., Navon, O., Kipnis, M., \& Mamlok-Naaman, R. (2005). Developing students' ability to ask more and better questions resulting from inquiry-type chemistry laboratories. Journal of Research in Science Teaching, 42(7), 791-806.

Hubble, E. (1954). The nature of science and other lectures. Wesport, CT: Greenwood Press, Publishers.

Irwin-Beck, D.J. (2002). National board certification: A descriptive study on its impact as a professional development activity. (Doctoral Dissertation, Boston College, 2002) Dissertation Abstracts International, 63, 444.

Jeanpierre, B., Oberhauser, K., \& Freeman, C. (2005). Characteristics of professional development that effect change in secondary science teachers' classroom practices. Journal of Research in Science Teaching, 42(6), 668-690.

Johnson, C.C. (2006). Effective professional development and change in practice: Barriers science teachers encounter and implications for reform. School Science \& Mathematics, 106(3), 150-161.

Johnson, M.A., \& Lawson, A.E. (1998). What are the relative effects of reasoning ability and prior knowledge on biology achievement in expository and inquiry classes? Journal of Research in Science Teaching, 35(1), 89-103.

Jöreskog, K., \& Sörbom, D. (2006). Lisrel (Version 8.80) [Computer Program]. Lincolnwood, IL: Scientific Software International.

Kahle, J.B., Meece, J., \& Scantlebury, K. (2000). Urban african-american middle school science students: Does standards-based teaching make a difference? Journal of Research in Science Teaching, 37(9), 1019-1041.

Kant, E. (1781/1998). Critique of pure reason (P. Guyer \& A.W. Wood, Trans.). Cambridge: Cambridge University Press.

Keedy, J. (1990). Traditional norms of schooling and the issue of organization change. Planning \& Changing, 21, 140-145.

Keedy, J., \& Robbins, A. (1993). Teacher collegial groups: A culture-building strategy for department chairs. Clearing House, 66(3), 185-188. 
Kelly, G.J., \& Chen, C. (1999). The sound of music: Constructing science as sociocultural practices through oral and written discourse. Journal of Research in Science Teaching, 36(8), 883-915.

Kitcher, P. (1995). The cognitive functions of scientific rhetoric. In H. Krips, McGuire, J.E., and Melia, T. (Ed.), Science, reason, and rhetoric. Pittsburgh: University of Pittsburgh Press.

Kuhn, D., Black, J., \& Keselman, A. (2000). The development of cognitive skills to support inquiry learning. Cognition and Instruction, 18(4), 495-523.

Kuhn, T.S. (1962/1996). The structure of scientific revolutions (3rd ed.). Chicago: The University of Chicago Press.

Larochelle, M., \& Bednarz, N. (1998). Constructivism and education: Beyond epistemological correctness. In Larochelle, M., Bednarz, N., and Garrison, J. (Eds.), Constructivism and education. (pp.3-22) Cambridge: Cambridge University Press.

Lawrenz, F., Huffman, D., \& Appleldoorn, K. (2002). CETP core evaluation classroom observation handbook. Minneapolis, MN: University of Minnesota.

Lawson, A.E. (1978). The development and validation of a classroom test of formal reasoning. Journal of Research in Science Teaching, 15(1), 11-24.

Lawson, A.E., \& Bealer, J.M. (1984). The acquisition of basic quantitative reasoning skills during adolescence: Learning or development? Journal of Research in Science Teaching, 21(4), 417-423.

Lawson, A.E., Costenson, K., \& Cisneros, R. (1986). A summary of research in science education--1984. Science Education, 70(6), 191-346.

Lawson, A.E., Lawson, D.I., \& Lawson, C.A. (1984). Proportional reasoning and the linguistic abilities required for hypothetico-deductive reasoning. Journal of Research in Science Teaching, 21(2), 119-131.

Lederman, N.G. (1992). Students' and teachers' conceptions of the nature of science: A review of the research. Journal of Research in Science Teaching, 29(4), 331-359.

Lederman, N.G. (1999). Teachers' understanding of the nature of science and classroom practice: Factors that facilitate or impede the relationship. Journal of Research in Science Teaching, 36(8), 916-929.

Lederman, N.G., Abd-El-Khalick, F., \& Bell, R.L. (2002). Views of nature of science questionnaire: Toward valid and meaningful assessment of learners' conceptions of nature of science. Journal of Research in Science Teaching, 39(6), 497-521. 
Leonard, W.H., \& Chandler, P.M. (2003). Where is the inquiry in biology textbooks? The American Biology Teacher, 65(7), 485-487.

Levin, R.A. (1991). The debate over schooling: Influences of Dewey and Thorndike. Childhood Education, 68 (2), 71-75.

Liang, L.L., Chen, S., Chen, X., Kaya, O.N., Adams, A.D., Macklin, M., et al. (2006). Student understanding of science and science inquiry (SUSSI): Revision and further validation of an assessment instrument, Annual Conference of the National Association for Research in Science Teaching (NARST). San Francisco, CA, April 3-6, 2006.

Loewenberg Ball, D., \& Bass, H. (2000). Making believe: The collective construction of public mathematical knowledge in the elementary classroom. In D.C. Philips (Ed.), Constructivism in education: Opinions and second opinions on controversial issues (pp. 193-224). Chicago: The University of Chicago Press.

Lortie, D.C. (1975). Schoolteacher. Chicago: The University of Chicago Press.

Loucks-Horsley, S., Hewson, P.W., Love, N., \& Stiles, K. (1998) Designing professional development for teachers of science and mathematics. Thousand Oaks: Corwin Press.

Lumpe, A.T., \& Beck, J. (1996). A profile of high school biology textbooks using scientific literacy recommendations. The American Biology Teacher, 58(3), 147153.

Lustick, D. (2002, April). National board certification as professional development: A study that identifies a framework and findings of teachers learning to manage complexity, uncertainty and community. Paper presented at the Annual Meeting of the American Educational Research Association. New Orleans, LA, April 1-5, 2002 .

Lustick, D., \& Sykes, G. (2006). National board certification as professional development: What are teachers learning? Education Policy Analysis Archives, $14(5), 1-43$.

Lyne, J. (1995). Rhetoric in the context of scientific rationality. In H. Krips, McGuire, J.E., and Melia, T. (Ed.), Science, reason, and rhetoric. Pittsburgh: University of Pittsburgh Press.

Marlow, M.P., \& Stevens, E. (1999, March). Science teachers attitudes about inquirybased science. Paper presented at the Annual Conference of the National Association for Research in Science Teaching. Boston, MA, March 28-31, 1999.

Marx, K. (1926). Capital; a critique of political economy. Chicago: C. H. Kerr \& Company. 
Mitchell, J., \& Williams, S.E. (1993, April). Expert/novice difference in teaching with technology. Paper presented at the Annual Meeting of the American Educational Research Association. Atlanta, GA, April 12-16, 1993. (ERIC Document Reproduction Service No. ED364192)

Monk, D., \& King-Rice, J. (1994). Multi-level teacher resource effects on pupil performance in secondary mathematics and science: The role of teacher subject matter preparation. In R. Ehrenberg (Ed.), Choices and consequences: Contemporary policy issues in education (pp. 29-58). Ithaca, NY: ILR Press.

Morf, A. (1998). An epistemology for didactics: Speculations on situating a concept. In M. Larochelle, Bednarz, N., and Garrison, J. (Ed.), Constructivism in education (pp. 29-42). Cambridge: Cambridge University Press.

National Board for Professional Teaching Standards. (2001). Current candidate survey, all candidates. Retrieved on July 12, 2006, from http://www.nbpts.org/pdf/cert_allcand_survey.pdf.

National Board for Professional Teaching Standards. (2002). What teachers should know and be able to do. Arlington, VA: Author.

National Board for Professional Teaching Standards. (2003a). Adolescence and young adult science portfolio instructions. Arlington, VA: Author.

National Board for Professional Teaching Standards. (2003b). Early adolescence science standards. Arlington, VA: Author.

National Board for Professional Teaching Standards. (2004a). Adolescence and young adult science portfolio instructions. Arlington, VA: Author.

National Board for Professional Teaching Standards. (2004b). Early adolescence science portfolio instructions. Arlington, VA: Author.

National Board for Professional Teaching Standards. (2006a). About NBPTS. Retrieved June 2, 2006, from http://www.nbpts.org/about/index.cfm.

National Board for Professional Teaching Standards. (2006). Standards development. Retrieved December 28, 2006 from http://www.nbpts.org/the_standards/standards_development.

National Board for Professional Teaching Standards. (2006c). Creation of NBPTS. Retrieved May 31, 2006, from http://www.nbpts.org/about/hist.cfm.

National Board for Professional Teaching Standards. (2006d). Summary of peer reviews of SAS study. Retrieved on June 13, 2006, from

http://www.nbpts.org/pdf/summary_of_peer-reviews_of_sanders_study.pdf. 
National Board for Professional Teaching Standards. (2007). State and local information. Retrieved July 8, 2007, from http://www.nbpts.org/resources/state_local_information.

National Commission on Excellence in Education. (1983). A nation at risk: The imperative for educational reform. Washington, D.C.: Author.

National Center for Educational Statistics. (1988). Teacher questionnaire NELS: 88 base year. In U. S. Deptartment of Education. Retrieved on July 23, 2006, from http://nces.ed.gov/surveys/nels88/questionnaires.asp.

National Research Council. (1996). National science education standards. Washington D.C.: National Academies Press.

National Research Council. (2000). How people learn : Brain, mind, experience, and school. Bransford, J.D., Brown, A.L. \& Cocking, R.R. (Eds.). Washington, D.C.: National Academy Press.

National Research Council. (2000). Inquiry and the national science inquiry standards. Washington, D.C.: National Academy Press.

National Research Council. (2001). Physics in a new era: An overview. Retrieved November 8, 2006, from http://fermat.nap.edu/books/0309073421/html/125.html.

National Research Council. (2006). America's lab report: Investigations in high school science. Washington, D.C.: The National Academy Press.

Nolen, S.B. (2003). Learning environment, motivation, and achievement in high school science. Journal of Research in Science Teaching, 40(4), 347-368.

O'Connor, E.A. \& Fish, M.C. (1997). Differences between the classrooms of expert and novice teachers on the dimensions of the "Classroom systems observation scale." Paper presented at the Annual Meeting of the New England Educational Research Organization. Portsmouth, NH, April 30-May 2, 1997.

O'Sullivan, R., Hudson, M., Orsini, M., Arter, J., Stiggins, R., \& Iovacchini, L. (2005). Student achievement and performance. Chapel Hill, NC: University of North Carolina at Chapel Hill.

Panofsky, C.P., John-Steiner, V., \& Blackwell, P.J. (1990). The development of scientific concepts and discourse. In L. C. Moll (Ed.), Vygotsky and education: Instructional implications and applications of sociohistorical psychology ( $\mathrm{pp}$. 251-267). Cambridge: Cambridge University Press.

Phillips, D.C. (2000). An opinionated account of the constructivist landscape. In D. C. Phillips (Ed.), Constructivism in education: Opinions and second opinions on controversial issues. Chicago: The University of Chicago Press. 
Piburn, M., \& Sawada, D., (2000). Reformed Teaching Observation Protocol (RTOP). ACEPT Technical Report IN00-3. Retrieved on August 4, 2005 from http://PhysicsEd.BuffaloState.Edu/AZTEC/rtop/RTOP_full/PDF/.

Plato (trans. 1980). The laws of Plato / translated with notes and an interpretive essay by Thomas L. Pangle. New York: Basic Books.

Popper, K. (1962). Conjectures and refutations: The growth of scientific knowledge. New York: Basic Books.

Popper, K. (1968). The logic of scientific discovery. New York: Harper \& Row.

Popper, K. (1983). Realism and the aim of science. Totowa, NJ: Roman and Littlefield.

Ramey-Gassert, L., Shroyer, M.G., \& Staver, J.R. (1996). A qualitative study of factors influencing science teaching self-efficacy of elementary level teachers. Science Education, 80(3), 283-315.

Raudenbush, S.W. \& Bryk, A.S. (2002). Hierarchical linear models: Applications and data analysis methods (2nd ed.). Thousand Oaks, CA: Sage Publications.

Riggs, I., \& Enochs, L. (1990). Towards the development of an elementary teacher's science teaching efficacy belief instrument. Science Education, 74(6), 625-637.

Roehrig, G., \& Luft, J. (2004). Constraints experienced by beginning secondary science teachers in implementing scientific inquiry lessons. International Journal of Science Education, 26 (1), p.3-24.

Rossman, G., \& Rallis, S. (2003). Learning in the field: An introduction to qualitative research. (2nd ed.). Thousand Oaks, CA: Sage Publications.

Rotter, J.B. (1966). Generalized expectancies for internal versus external control of reinforcement. Psychological Monographs: General \& Applied, 80(1), 1-28.

Ruiz-Primo, M., Li, M., \& Shavelson, R. (2002). Looking into Students' Science Notebooks: What Do Teachers Do with Them? CSE Technical Report. (No. CSETR-562). Washington, D.C.: Office of Educational Research and Improvement.

Russell, B. (1905). On denoting. Mind, 14(56), 479-493.

Russell, B. (1914). Our knowledge of the external world as a field for scientific method in philosophy. Chicago: The Open court publishing company.

Sanders, M. (1993). Erroneous ideas about respiration: The teacher factor. Journal of Research in Science Teaching, 30(8), 919-934. 
Sanders, W.L., Ashton, J.J., \& Wright, S.P. (2006). Comparison of the effects of NBPTS certified teachers with other teachers on the rate of student academic progress. Retrieved on June 12, 2006 from http://www.nbpts.org/pdf/sas_final_report.pdf.

Saderholm, J. (2006, April). National Board Certification as a change agent for science teachers. Paper presented at the annual meeting of the National Association for Research in Science Teaching, San Francisco, CA. April 3-6, 2006.

Schon, D.A. (1987). Educating the reflective practitioner. San Francisco: Jossey-Bass Publishers.

Schwab, J. (1962a). The teaching of science as enquiry. Cambridge, MA: Harvard University Press.

Schwab, J. (1962b). Biology teachers' handbook. New York: Wiley.

Schwartz, R.S., \& Lederman, N.G. (2002). "It's the nature of the beast": The influence of knowledge and intentions on learning and teaching nature of science. Journal of Research in Science Teaching, 39(3), 205-236.

Seife, C. (2000). Scientific priority: CERN's gamble shows perils, rewards of playing the odds. Science, 289(5488), 2260-2262.

Serafini Jr., F. (2001). A case study of a national board for professional teaching standards candidate's experience. Unpublished Dissertation, Arizona State University.

Shadish, W.R., Cook, T.D., \& Campbell, D.T. (2002). Experimental and quasiexperimental designs for generalized causal inference. Boston: Houghton Mifflin.

Smith, T.W., Gordon, B., Colby, S.A., \& Wang, J. (2005). An examination of the relationship between the depth of student learning and national board certification status. Retrieved on June 9, 2006 from http:/www.news.appstate.edu/releases/091905NBPTS\%20Manuscrip.pdf.

Spencer, H. (1864). Education: Intellectual, moral, and physical. New York: Appleton.

SPSS, Inc. (2005). SPSS for Windows. (Version 14.0). Chicago: SPSS, Inc.

Stevens, J.P. (2002). Applied multivariate statistics for the social sciences (Vol. 4). Mahwah, NJ: Lawrence Erlbaum, Inc.

Stone, J.E. (2002). The value-added achievement gains of NBPTS-certified teachers in Tennessee: A brief report. Johnson City, TN: College of Education, East Tennessee State University. 
Suits, J.P. (2005). Assessing investigative skill development in inquiry-based and traditional college science laboratory courses. School Science and Mathematics, 104(6), 248-257.

Supovitz, J.A., \& Turner, H.M. (2000). The effects of professional development on science teaching practices and classroom culture. Journal of Research in Science Teaching, 37(9), 963-980.

Supovitz, J.A., Mayer, D.P., \& Kahle, J.B. (2000). Promoting inquiry-based instructional practice: The longitudinal impact of professional development in the context of systemic reform. Educational Policy, 14(3), 331-356.

Tashakorri, A., \& Teddlie, C. (1998). Mixed methodology: Combining qualitative and quantitative approaches. Thousand Oaks, CA: Sage Publications.

Taylor, G. (2000). Teacher change and the national board for professional teaching standards: A case study of eleven Colorado teachers. Unpublished Dissertation, University of Colorado.

Thorndike, E.L. (1901). The influence of improvement in one mental function upon the efficiency of other functions (I). Psychological Review, 8, 247-261.

Thorndike, E.L. (1922). The psychology of arithmetic. New York: The MacMillan Company.

Tobin, K. (1998). Sociocultural perspectives on the teaching and learning of science. In Larochelle, M., Bednarz, N., and Garrison, J. (Ed.), Constructivism in education (pp. 195-212). Cambridge: Cambridge University Press.

Tobin, K.G., Roth, W-M., \& Zimmermann, A. (2001). Learning to teach science in urban schools. Journal of Research in Science Teaching, 38(8), 941-964.

Toulmin, S. (1995). Science and the many faces of rhetoric. In H. Krips, McGuire, J.E., and Melia, T. (Ed.), Science, reason, and rhetoric. Pittsburgh: University of Pittsburgh Press.

Tretter, T.R., \& Jones, M.G. (2003). Relationships between inquiry-based teaching and physical science standardized test scores. School Science and Mathematics, 103(7), 345-350.

Tretter, T.R., Jones, M.G., Andre, T., Negishi, A., \& Minogue, J. (2006). Conceptual boundaries and distances: Students' and experts' concepts of the scale of scientific phenomena. Journal of Research in Science Teaching, 43(3), 282-319.

Tudge, J. (1990). Vygotsky, the zone of proximal development, and peer collaboration: Implications for classroom practice. In L. C. Moll (Ed.), Vygotsky and education: Instructional implications and applications of sociohistorical psychology. Cambridge: University of Cambridge Press. 
Union of Concerned Scientists. (2006). FDA scientists pressured to exclude, alter findings; scientists fear retaliation for voicing safety concerns. Retrieved August 7, 2006, from http://www.ucsusa.org/news/press_release/fda-scientistspressured.html?

U.S. Census Bureau. (2000). About metropolitan and micropolitan statistical areas. Retrieved March 4, 2007, from http://www.census.gov/population/www/estimates/aboutmetro.html.

Vandevoort, L.G., Amrein-Beardsley, A., \& Berliner, D.C. (2004). National board certified teachers and their students' achievement, Education Policy Analysis Archives (Vol. 12): Arizona State University \& University of South Florida.

Van Driel, J.H., \& Verloop, N. (2002). Experienced teachers' knowledge of teaching and learning of models and modeling in science education. International Journal of Science Education, 24(12), 1255-1272.

Von Glaserfeld, E. (2005). Introduction: Aspects of constructivism. In C. Twomey Fosnot (Ed.), Constructivism: Theory, perspectives, and practice. New York: Teachers College Press.

Von Secker, C.E., \& Lissitz, R.W. (1999). Estimating the impact of instructional practices on student achievement in science. Journal of Research in Science Teaching, 36(10), 1110-1136.

Von Secker, C.E. (2002). Effects of inquiry-based teacher practices on science excellence and equity. The Journal of Educational Research, 95(3), 151-160.

Vygotsky, L.S. (1978). Mind in society: The development of higher psychological processes Cambridge: Harvard University Press.

Weber, M. (1958). The protestant ethic and the spirit of capitalism (T. Parsons, Trans.). New York: Scribner.

Wenning, C.J. (2005a). Minimizing resistance to inquiry-oriented science instruction: The importance of climate setting. Journal of Physics Teacher Education Online, $3(2), 10-15$.

Wenning, C.J. (2005b). Levels of inquiry: Hierarchies of pedagogical practice and inquiry processes. Journal of Physics Teacher Education Online, 2(3), 3-11.

Whitley, R. (2000). The intellectual and social organization of the sciences (2nd ed.). Oxford: Oxford University Press.

Wolpert, L. (1993). The unnatural nature of science. Cambridge, MA: Harvard University Press. 
Zimmerman, B.J., \& Cleary, T.J. (2006). Adolescents' development of personal agency: The role of self-efficacy beliefs and self-regulatory skill. In Tim Urdan and Frank Pajares (Eds.), Self-efficacy beliefs of adolescents (pp. 45-69). Greenwich, CT: Information Age Publishing. 
APPENDICES 
Appendix A

\begin{tabular}{|c|c|c|c|}
\hline \multicolumn{4}{|l|}{ Research Question-Data Source-Analytic Method Matrix } \\
\hline Question: & Data Source & Analytic Method & Research Question \\
\hline $\begin{array}{l}\text { Do NBCTs have a higher level of NOS understanding than their non- } \\
\text { NBCT counterparts? } \\
\text { Does experience explain differences in teacher NOS understanding? }\end{array}$ & $\begin{array}{l}\text { SUSSI-teacher data } \\
\text { Teacher Group }\end{array}$ & ANOVA & $\begin{array}{l}\text { 1) Main question } \\
\text { 1) Explains } \\
\text { counterfactual } \\
\end{array}$ \\
\hline $\begin{array}{l}\text { - Do NBCTs have a higher level of NOS understanding than their non- } \\
\text { NBCT counterparts? }\end{array}$ & SUSSI-teacher data & $\begin{array}{l}\text { Qualitative analysis } \\
\text { teacher responses }\end{array}$ & 1) Main question \\
\hline $\begin{array}{l}\text { Do NBCTs enact science inquiry pedagogical practice more frequently } \\
\text { than their non-NBCT counterparts? } \\
\text { Does experience explain differences in teacher science inquiry } \\
\text { pedagogical practice? }\end{array}$ & $\begin{array}{l}\text { ESIS-teacher data } \\
\text { Teacher group }\end{array}$ & MANOVA & $\begin{array}{l}\text { 2) Main question } \\
\text { 4) Explains } \\
\text { counterfactual } \\
\end{array}$ \\
\hline $\begin{array}{l}\text { - Do the students perceive the activity in their classroom in the same way } \\
\text { their teachers perceive it? }\end{array}$ & $\begin{array}{l}\text { ESIS-student data } \\
\text { ESIS-teacher data }\end{array}$ & $\begin{array}{l}\text { Correlation, Factor } \\
\text { Analysis }\end{array}$ & $\begin{array}{l}\text { Addresses self-report } \\
\text { bias }\end{array}$ \\
\hline $\begin{array}{l}\text { Do the students of NBCTs display greater change in scientific } \\
\text { reasoning skills than the students of their non-NBCT counterparts? }\end{array}$ & $\begin{array}{l}\text { LTSR-student data } \\
\text { Teacher group }\end{array}$ & MANOVA & 3) Main question \\
\hline $\begin{array}{l}\text { - Do the students of NBCTs display greater change in comprehension of } \\
\text { NOS concepts than the students of their non-NBCT counterparts? } \\
\text { Do the students of NBCTs display greater change in comprehension of } \\
\text { NOS concepts than the students of their non-NBCT counterparts? }\end{array}$ & $\begin{array}{l}\text { SUSSI-student data } \\
\text { Teacher group } \\
\text { SUSSI-student data }\end{array}$ & MANOVA & $\begin{array}{l}\text { 3) Main question } \\
\text { 3) Main question }\end{array}$ \\
\hline $\begin{array}{l}\text { Do course documents created by NBCTs support higher level } \\
\text { engagement of science inquiry concepts than those of their non-NBCT } \\
\text { counterparts? }\end{array}$ & Course documentation & $\begin{array}{l}\text { Documentary } \\
\text { analysis }\end{array}$ & Triangulation \\
\hline \multirow{2}{*}{$\begin{array}{l}\text { - Do classroom observations of NBCTs show a higher science inquiry } \\
\text { teaching practice than those of their non-NBCT counterparts? }\end{array}$} & $\begin{array}{l}\text { Observation using RTOP } \\
\text { classroom observation } \\
\text { protocols }\end{array}$ & Qualitative analysis & Triangulation \\
\hline & $\begin{array}{l}\text { Observation using CETP } \\
\text { classroom observation } \\
\text { protocols }\end{array}$ & Qualitative analysis & Triangulation \\
\hline
\end{tabular}


Appendix B

\begin{tabular}{|c|c|c|c|c|c|c|}
\hline $\begin{array}{l}\text { ESIS-B } \\
\text { Teacher Questionnaire }\end{array}$ & $\begin{array}{l}\text { Never } \\
\text { or not } \\
\text { at all }\end{array}$ & Rarely & & & $\begin{array}{l}\text { Freq- } \\
\text { uently }\end{array}$ & $\begin{array}{l}\text { Exclus } \\
\text { ively }\end{array}$ \\
\hline \multicolumn{7}{|c|}{$\begin{array}{l}\text { During science lessons, on average, to what extent does a typical individual student in your } \\
\text { classroom: }\end{array}$} \\
\hline $\begin{array}{l}\text { 1. memorize scientific facts or information } \\
\text { independently? }\end{array}$ & 0 & 0 & 0 & 0 & 0 & 0 \\
\hline $\begin{array}{l}\text { 2. use data to construct a reasonable } \\
\text { explanation? }\end{array}$ & 0 & 0 & 0 & 0 & 0 & 0 \\
\hline $\begin{array}{l}\text { 3. seek and recognize patterns (trends in the } \\
\text { data or observations)? }\end{array}$ & 0 & 0 & 0 & 0 & 0 & 0 \\
\hline $\begin{array}{l}\text { 4. detect sequences (the order of events in a } \\
\text { science activity)? }\end{array}$ & 0 & 0 & 0 & 0 & 0 & 0 \\
\hline $\begin{array}{l}\text { 5. follow a set series of steps to get the right } \\
\text { answer to a question? }\end{array}$ & 0 & 0 & 0 & 0 & 0 & 0 \\
\hline \multicolumn{7}{|c|}{$\begin{array}{l}\text { (Note: the word investigation in this survey refers to student activity: describing, classifying, } \\
\text { and experimenting with objects, events, and organisms.) }\end{array}$} \\
\hline & Never & $\begin{array}{c}\text { Once } \\
\text { per } \\
\text { week }\end{array}$ & $\begin{array}{c}\text { Twice } \\
\text { per } \\
\text { week }\end{array}$ & $\begin{array}{l}\text { 3x per } \\
\text { week }\end{array}$ & $\begin{array}{l}4 \times \text { per } \\
\text { week }\end{array}$ & $\begin{array}{c}5 \mathrm{x} \text { or } \\
\text { more } \\
\text { per } \\
\text { week }\end{array}$ \\
\hline $\begin{array}{l}\text { 6. ask questions during investigations that } \\
\text { lead to further ideas, questions and } \\
\text { investigations? }\end{array}$ & 0 & 0 & 0 & 0 & 0 & 0 \\
\hline $\begin{array}{l}\text { 7. wait to act until the teacher gives } \\
\text { instructions for the next step in the } \\
\text { investigation? }\end{array}$ & 0 & 0 & 0 & 0 & 0 & 0 \\
\hline $\begin{array}{l}\text { 8. use science process skills (e.g., observing, } \\
\text { classifying, recording data) connected to a } \\
\text { specific science topic? }\end{array}$ & 0 & 0 & 0 & 0 & 0 & 0 \\
\hline $\begin{array}{l}\text { 9. choose appropriate tools for an } \\
\text { investigation? }\end{array}$ & 0 & 0 & 0 & 0 & 0 & 0 \\
\hline $\begin{array}{l}\text { 10. wait for the teacher's explanation before } \\
\text { expressing an observation or conclusion? }\end{array}$ & 0 & 0 & 0 & 0 & 0 & 0 \\
\hline $\begin{array}{l}\text { 11. offer explanations from previous } \\
\text { experiences and from knowledge gained } \\
\text { during investigations? }\end{array}$ & 0 & 0 & 0 & 0 & 0 & 0 \\
\hline $\begin{array}{l}\text { 12. make connections to previously held ideas } \\
\text { (or revise previous } \\
\text { conceptions/assumptions)? }\end{array}$ & 0 & 0 & 0 & 0 & 0 & 0 \\
\hline $\begin{array}{l}\text { 13. communicate investigations and } \\
\text { explanations (purposes, procedures, } \\
\text { and/or results of investigations) to others? }\end{array}$ & 0 & 0 & 0 & 0 & 0 & 0 \\
\hline $\begin{array}{l}\text { 14. use investigation to satisfy their own } \\
\text { questions? }\end{array}$ & 0 & 0 & 0 & 0 & 0 & 0 \\
\hline $\begin{array}{l}\text { 15. listen carefully to peers as they discuss } \\
\text { scientific investigations? }\end{array}$ & 0 & 0 & 0 & 0 & 0 & 0 \\
\hline
\end{tabular}


Appendix B

\begin{tabular}{|c|c|c|c|c|c|c|}
\hline & Never & $\begin{array}{c}\text { Once } \\
\text { per } \\
\text { week }\end{array}$ & $\begin{array}{c}\text { Twice } \\
\text { per } \\
\text { week }\end{array}$ & $\begin{array}{c}3 \times \text { per } \\
\text { week }\end{array}$ & $\begin{array}{c}4 \times \text { per } \\
\text { week }\end{array}$ & $\begin{array}{l}5 \mathrm{x} \text { or } \\
\text { more per } \\
\text { week }\end{array}$ \\
\hline $\begin{array}{l}\text { 16. use drawing, graphing, or charting to } \\
\text { convey new information from a science } \\
\text { activity? }\end{array}$ & 0 & O & 0 & 0 & 0 & 0 \\
\hline $\begin{array}{l}\text { 17. show reluctance to ask questions that } \\
\text { might extend an activity? }\end{array}$ & 0 & O & 0 & O & 0 & 0 \\
\hline 18. assess their own or their group's work? & 0 & 0 & $\mathrm{O}$ & 0 & $\mathrm{O}$ & 0 \\
\hline $\begin{array}{l}\text { 19. use computers or calculators while } \\
\text { conducting investigations? }\end{array}$ & 0 & 0 & 0 & 0 & 0 & 0 \\
\hline \multicolumn{7}{|l|}{ To what extent do you: } \\
\hline $\begin{array}{l}\text { 20. use a textbook as the primary method for } \\
\text { studying science? }\end{array}$ & 0 & 0 & 0 & O & 0 & O \\
\hline $\begin{array}{l}\text { 21. use open-ended questions that encourage } \\
\text { observation, investigations, and scientific } \\
\text { thinking? }\end{array}$ & 0 & 0 & 0 & 0 & 0 & $\mathrm{O}$ \\
\hline $\begin{array}{l}\text { 22. identify questions that can be investigated } \\
\text { at varying levels of sophistication? }\end{array}$ & 0 & 0 & 0 & 0 & 0 & 0 \\
\hline $\begin{array}{l}\text { 23. encourage students to initiate further } \\
\text { investigation? }\end{array}$ & 0 & 0 & 0 & 0 & 0 & 0 \\
\hline $\begin{array}{l}\text { 24. ask a question or conduct an activity that } \\
\text { calls for a single correct answer? }\end{array}$ & 0 & 0 & O & 0 & 0 & 0 \\
\hline $\begin{array}{l}\text { 25. carefully listen to student ideas and } \\
\text { comments during science lessons? }\end{array}$ & 0 & 0 & O & 0 & 0 & 0 \\
\hline $\begin{array}{l}\text { 26. orchestrate and encourage student } \\
\text { dialogue about science? }\end{array}$ & 0 & 0 & 0 & 0 & 0 & 0 \\
\hline $\begin{array}{l}\text { 27. encourage students to defend the } \\
\text { adequacy or logic of statements and } \\
\text { finding? }\end{array}$ & 0 & 0 & O & 0 & 0 & 0 \\
\hline $\begin{array}{l}\text { 28. conclude an inquiry with the result of one } \\
\text { experiment? }\end{array}$ & 0 & 0 & 0 & O & 0 & $\mathrm{O}$ \\
\hline $\begin{array}{l}\text { 29. make readily available to students a wide } \\
\text { variety of resource materials for } \\
\text { investigations? }\end{array}$ & 0 & 0 & 0 & 0 & 0 & 0 \\
\hline
\end{tabular}

Teacher background questions

30. Please list your non-education college degrees and the subject in which they were awarded.

BA/BS:

O MA/MS:

O Ph.D/Ed.D:

31. Please check your teaching certification rank and list the subject area and grade levels you are certified to teach.

O Rank III:

Rank II:

O Rank: 


\section{Appendix B}

32. Are you NBPTS certified? If so, please list your certificate name (e.g. AYA/Science) and the year you received certification.

$\bigcirc$ Yes $O$ No Certificate: Year Certified:

33. How many years have you taught prior to this school year?

34. How many graduate science courses have you taken?

None or 2 courses 3 courses 4 courses 5 courses More than 5 courses
1 course

35. To what extent did your college science courses emphasize scientific inquiry (as inquiry is described in the above questions and in the vignette you read)? In other words, to what extent did you while a student conduct scientific inquiry in your college science courses? $\begin{array}{llllll}0 & 1 & 2 & 3 & 4 & 5\end{array}$

Not at all, in any

science course

Some inquiry, in some

All
courses
were
very
inquiry-
based

36. How many workshops on science education have you taken in the last five years?

$\begin{array}{ccccc}\text { None } & \begin{array}{c}\text { One } \\ \text { workshop }\end{array} & \begin{array}{c}\text { Two } \\ \text { workshops }\end{array} & \begin{array}{c}\text { Three } \\ \text { workshops }\end{array} & \begin{array}{c}\text { Four } \\ \text { workshops }\end{array}\end{array} \quad$ More than 4 workshops

37. Have you ever conducted or assisted with scientific research?

$\begin{array}{lllll}0 & 1 & 2 & 3 & 4\end{array}$

Never Some involvement

45

Yes, I have more than

once

38. How familiar are you with the 1996 National Science Education Standards?

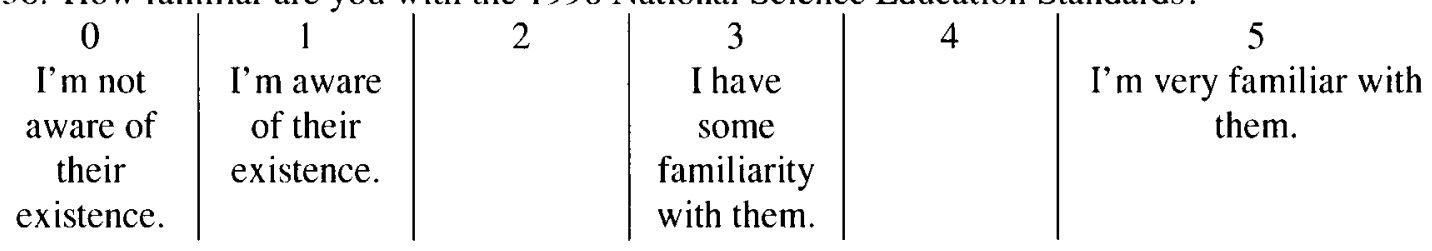

39. How familiar are you with the Kentucky Program of Studies?

\begin{tabular}{|c|c|c|c|c|c|}
\hline $\begin{array}{c}\text { o } \\
\text { I'm not } \\
\text { aware of its } \\
\text { existence. }\end{array}$ & $\begin{array}{l}1 \\
\text { I'm aware } \\
\text { of its } \\
\text { existence. }\end{array}$ & 2 & $\begin{array}{c}3 \\
\text { I have } \\
\text { some } \\
\text { familiarity } \\
\text { with it. }\end{array}$ & 4 & $\begin{array}{c}5 \\
\text { I'm very familiar with } \\
\text { it. }\end{array}$ \\
\hline
\end{tabular}


40. How familiar are you with the Kentucky Core Content for Assessment?

\begin{tabular}{|c|c|c|c|c|}
\hline $\begin{array}{c}0 \\
\text { I'm not } \\
\text { aware of its } \\
\text { existence. }\end{array}$ & $\begin{array}{l}1 \\
\text { I'm aware of } \\
\text { its existence. }\end{array}$ & 2 & $\begin{array}{c}3 \\
\text { I have some } \\
\text { familiarity } \\
\text { with it. }\end{array}$ & $\begin{array}{c}5 \\
\text { I'm very } \\
\text { familiar with } \\
\text { it. }\end{array}$ \\
\hline
\end{tabular}

40. Please rank the three most important factors in determining your science curriculum content (what science topics you actually teach)? Mark " 1 " next to the one that has the most influence, etc.

District curriculum

State or national standards

Textbook

Available instructional materials

Guidance from principal

Other teachers' input

Other (specify)

41. To what extent do you collaborate with other teachers in the planning of science lessons?

0

Not at all
2

Some collaboration
4

Regular collaboration

42. What are the factors that limit your use of the inquiry method of teaching science? Check all that apply.

There is nothing that limits the use of inquiry in my classroom

Lack of experience with inquiry, lack of awareness of inquiry

Reluctance to give up the role of primary source of classroom information

There isn't enough time

Have tried it and it wasn't successful

Doubts about students' capability to do inquiry

Concern about discipline, large class size, potential chaos, etc.

Insufficient time and support for implementation

Insufficient background in science

Disagreement with the notion that inquiry is necessary

Don't have the necessary materials

Lack of parent and community support

Parental concerns about nonuse of science textbooks

Other (please specify in the space below) 


\section{Appendix C}

Student Understanding of Science and Scientific Inquiry (SUSSI)

Please read EACH statement carefully, and then indicate the degree to which you agree or disagree with EACH statement by circling the appropriate letters to the right of each statement.

\section{Observations and Inferences}

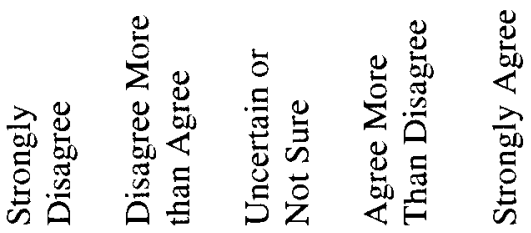
A. Scientists' observations of the same event may be different because the scientists' prior knowledge may
SD
D
$\mathrm{U}$
A $\quad$ SA affect their observations.
B. Scientists' observations of the same event will be the same because scientists are objective.

SD D U A SA
C. Scientists' observations of the same event will be the same because observations are facts.
SD $\quad \mathrm{D} \quad \mathrm{U} \quad \mathrm{A} \quad \mathrm{SA}$
D. Scientists' may make different interpretations based on the same observations.
SD $\quad$ D $\quad$ U $\quad$ A $\quad$ SA

With examples, explain why you think scientists' observations and interpretations are the same OR different.

\section{Change of Scientific Theories}

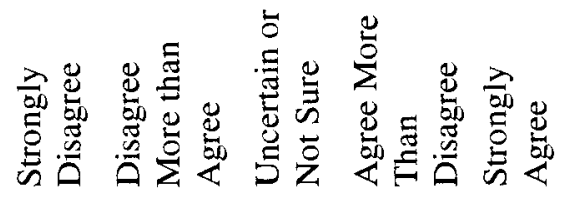
A. Scientific theories are subject to on-going testing and revision.
B. Scientific theories may be completely replaced by new theories in light of new evidence.
C. Scientific theories may be changed because scientists reinterpret existing observations.
D. Scientific theories based on accurate experimentation will not be changed.

$\begin{array}{lllll}\text { SD } & \text { D } & \text { U } & \text { A } & \text { SA } \\ \text { SD } & \text { D } & \text { U } & \text { A } & \text { SA } \\ \text { SD } & \text { D } & \text { U } & \text { A } & \text { SA } \\ \text { SD } & \text { D } & \text { U } & \text { A } & \text { SA }\end{array}$

With examples, explain why you think scientific theories do not change OR how (in what ways) scientific theories may be changed. 


\section{Appendix C}

Student Understanding of Science and Scientific Inquiry (SUSSI)

\section{Scientific Laws vs. Theories}

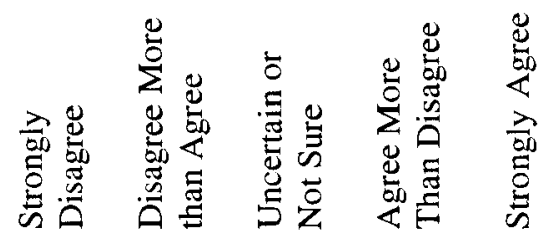
A. Scientific theories exist in the natural world and are
SD
D
$\mathrm{U}$
A SA
B. Unlike theories, scientific laws are not subject to change.
SD
D
$\mathrm{U}$
A SA
C. Scientific laws are theories that have been proven.
SD

D
$\mathrm{U}$
A SA
D. Scientific theories explain scientific laws.
SD
D
$\mathrm{U} \quad \mathrm{A}$
SA

With examples, explain the nature of and difference between scientific theories and scientific laws.

\section{Social and Cultural Influence of Science}

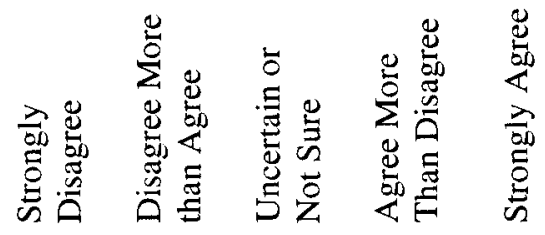

A. Scientific research is not influenced by society and culture because scientists are trained to conduct "pure", unbiased studies.

B. Cultural values and expectations determine what science is conducted and accepted.

$\begin{array}{lllll}\text { SD } & \text { D } & \text { U } & \text { A } & \text { SA } \\ \text { SD } & \text { D } & \text { U } & \text { A } & \text { SA } \\ \text { SD } & \text { D } & \text { U } & \text { A } & \text { SA } \\ \text { SD } & \text { D } & \text { U } & \text { A } & \text { SA }\end{array}$
and culture.

With examples, explain how society and culture affect OR do not affect scientific research. 


\section{Appendix C}

Student Understanding of Science and Scientific Inquiry (SUSSI)

\section{Imagination and Creativity in Scientific Investigations}

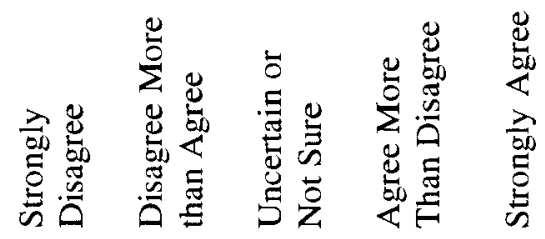
A. Scientists use their imagination and creativity when they collect data.
SD
D
U
A SA
B. Scientists use their imagination and creativity when they
SD
D
U
A SA
C. Scientists do not use their imagination and creativity because these conflict with their logical reasoning.
SD
D
U
A SA
D. Scientists do not use their imagination and creativity because these can interfere with objectivity.
SD

D U A SA

With examples, explain how and when scientists use imagination and creativity OR do not use imagination and creativity.

\section{Methodology of Scientific Investigation}

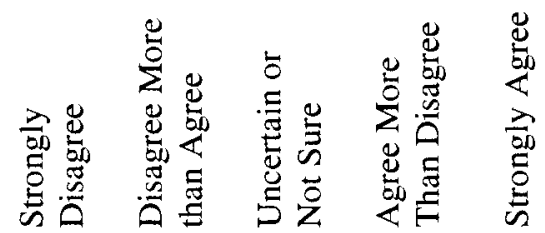

A. Scientists use different types of methods to conduct scientific investigations.

SD D

U

A SA

B. Scientists follow the same step-by-step scientific method.

SD

D

U

A SA

C. When scientists use the scientific method correctly, their SD

D

A

SA

D. Experiments are not the only means used in the development of scientific knowledge.

$\mathrm{SD}$

D

U

A

SA

With examples, explain whether scientists follow a single, universal scientific method $\mathbf{O R}$ use different types of methods. 


\section{CETP Classroom Observation Protocol}

Class Description and Purpose: Classroom Checklist

Fill in the types of instruction (not the instructor's actual activities, in case they are correcting papers or something non-instructional), student engagement, and cognitive activity use in each five-minute portion of this class in the boxes below. There may be one or more strategy used in each category during each interval. For example, SGD, HOA, and TIS often occur together in a five-minute period, but SGD and L do not.

\section{Type of Instruction}

\begin{tabular}{|c|c|c|c|}
\hline $\mathrm{L}$ & Lecture/presentation & $\mathrm{CL}$ & Cooperative learning (roles) \\
\hline PM & Problem modeling & $\mathrm{LC}$ & Learning center/station \\
\hline SP & Student presentation (formal) & TIS & Teacher interacting with student \\
\hline LWD & Lecture with demonstration & UT & Utilizing digital educational media \\
\hline $\mathrm{D}$ & Demonstration & & and/or technology \\
\hline $\mathrm{CD}$ & Class discussion & A & Assessment: please describe \\
\hline WW & Writing work (if in groups add SGD) & $\mathrm{AD}$ & Administrative tasks \\
\hline \multirow[t]{2}{*}{ RSW } & Reading seat work if in groups add SGD) & $\mathrm{OOC}$ & Out-of-class experience \\
\hline & & I & Interruption \\
\hline $\mathrm{HOA}$ & Hands-on activity/materials & OTH & Other: please describe \\
\hline SGD & Small group discussion (pairs count) & & \\
\hline
\end{tabular}

\section{Student Engagement}

LE low engagement, $80 \%$ or more of the students are off-task

$\mathrm{ME}$ mixed engagement

HE high engagement, $80 \%$ or more of the students are engaged

\section{Cognitive Activity}

1 Receipt of Knowledge (lectures, worksheets, questions, observing, homework)

2 Application of Procedural Knowledge (skill building, performance)

3 Knowledge Representation (organizing, describing, categorizing)

4 Knowledge Construction (higher order thinking, generating, inventing, solving problems, revising, etc.)

5 Other (e.g., classroom disruption)

\section{Time in Minutes}

\begin{tabular}{|l|l|l|l|l|l|l|l|l|l|l|l|l|}
\hline & $0-5$ & $5-10$ & $10-15$ & $15-20$ & $20-25$ & $25-30$ & $30-35$ & $35-40$ & $40-45$ & $45-50$ & $50-55$ & $55-60$ \\
\hline Instruction & & & & & & & & & & & & \\
& & & & & & & & & & & & \\
\hline Student & & & & & & & & & & & & \\
\hline Cognitive & & & & & & & & & & & & \\
\hline
\end{tabular}

\begin{tabular}{|l|l|l|l|l|l|l|l|l|l|l|l|l|}
\hline & $60-65$ & $65-70$ & $70-75$ & $75-80$ & $80-85$ & $85-90$ & $90-95$ & $95-100$ & $\begin{array}{c}100- \\
105\end{array}$ & $\begin{array}{c}105- \\
110\end{array}$ & $110-115$ & $115-120$ \\
\hline Instruction & & & & & & & & & & & & \\
\hline Student & & & & & & & & & & & & \\
\hline Cognitive & & & & & & & & & & & & \\
\hline
\end{tabular}


Appendix E

Reformed Teaching Observation Protocol (RTOP)

\author{
Daiyo Sawada \\ External Evaluator \\ Michael Piburn \\ Internal Evaluator \\ and
}

Kathleen Falconer, Jeff Turley, Russell Benford and Irene Bloom

Evaluation Facilitation Group (EFG)

Technical Report No. IN00-1

Arizona Collaborative for Excellence in the Preparation of Teachers

Arizona State University

\title{
I. BACKGROUND INFORMATION
}

Name of teacher:

Announced Observation?

explain)

(yes, no, or

Location of class:

(district, school, room)

Years of Teaching:

Teaching Certification:

(K-8 or 7-12)

Subject observed:

Grade level:

Observer:

Date of observation:

Start time:

End time:

\section{CONTEXTUAL BACKGROUND AND ACTIVITIES}

In the space provided below please give a brief description of the lesson observed, the classroom setting in which the lesson took place (space, seating arrangements, etc.), and any relevant details about the students (number, gender, ethnicity) and teacher that you think are important. Use diagrams if they seem appropriate. 
Appendix E

Record here events which may help in documenting the ratings.

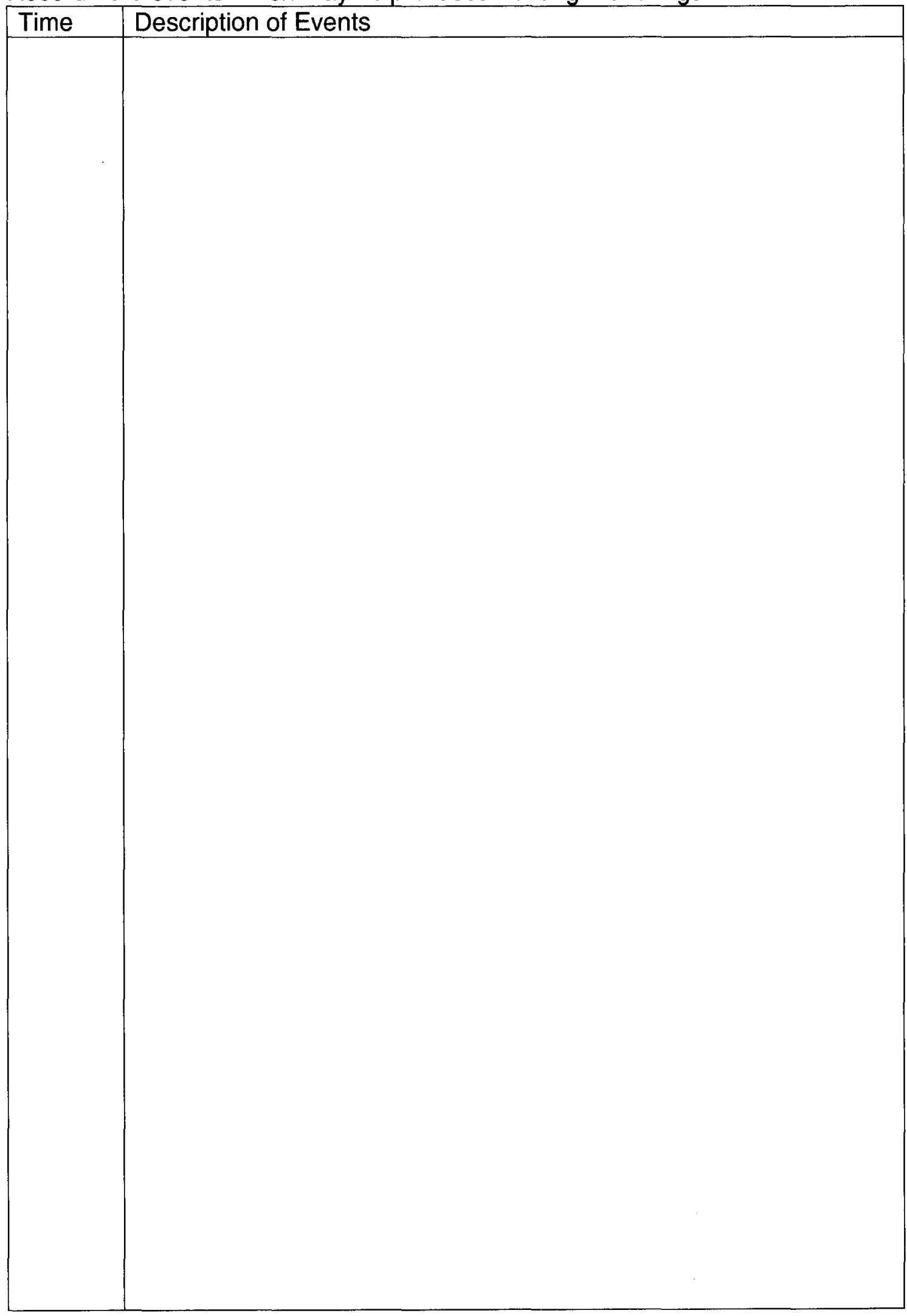


1) The instructional strategies and activities respected students' prior knowledge and the preconceptions inherent therein.

2) The lesson was designed to engage students as members of a learning community.

3) In this lesson, student exploration preceded formal presentation.

4) This lesson encouraged students to seek and value alternative modes of investigation or of problem solving.

5) The focus and direction of the lesson was often determined by ideas originating with students.

\section{CONTENT}

\section{Propositional knowledge}

6) The lesson involved fundamental concepts of the subject.

7) The lesson promoted strongly coherent conceptual understanding.
8) The teacher had a solid grasp of the subject matter content inherent in the lesson.

9) Elements of abstraction (i.e., symbolic representations, theory building) were encouraged when it was important to do so.

10) Connections with other content disciplines and/or real world phenomena were explored and valued.

\section{Procedural Knowledge}

11) Students used a variety of means (models, drawings, graphs, concrete materials, manipulatives, etc.) to represent phenomena.

12) Students made predictions, estimations and/or hypotheses and devised means for testing them.

13) Students were actively engaged in thought-provoking activity that often involved the critical assessment of procedures.

14) Students were reflective about their learning.

15) Intellectual rigor, constructive criticism, and the challenging of ideas were valued. 
Appendix E

Continue recording salient events here.

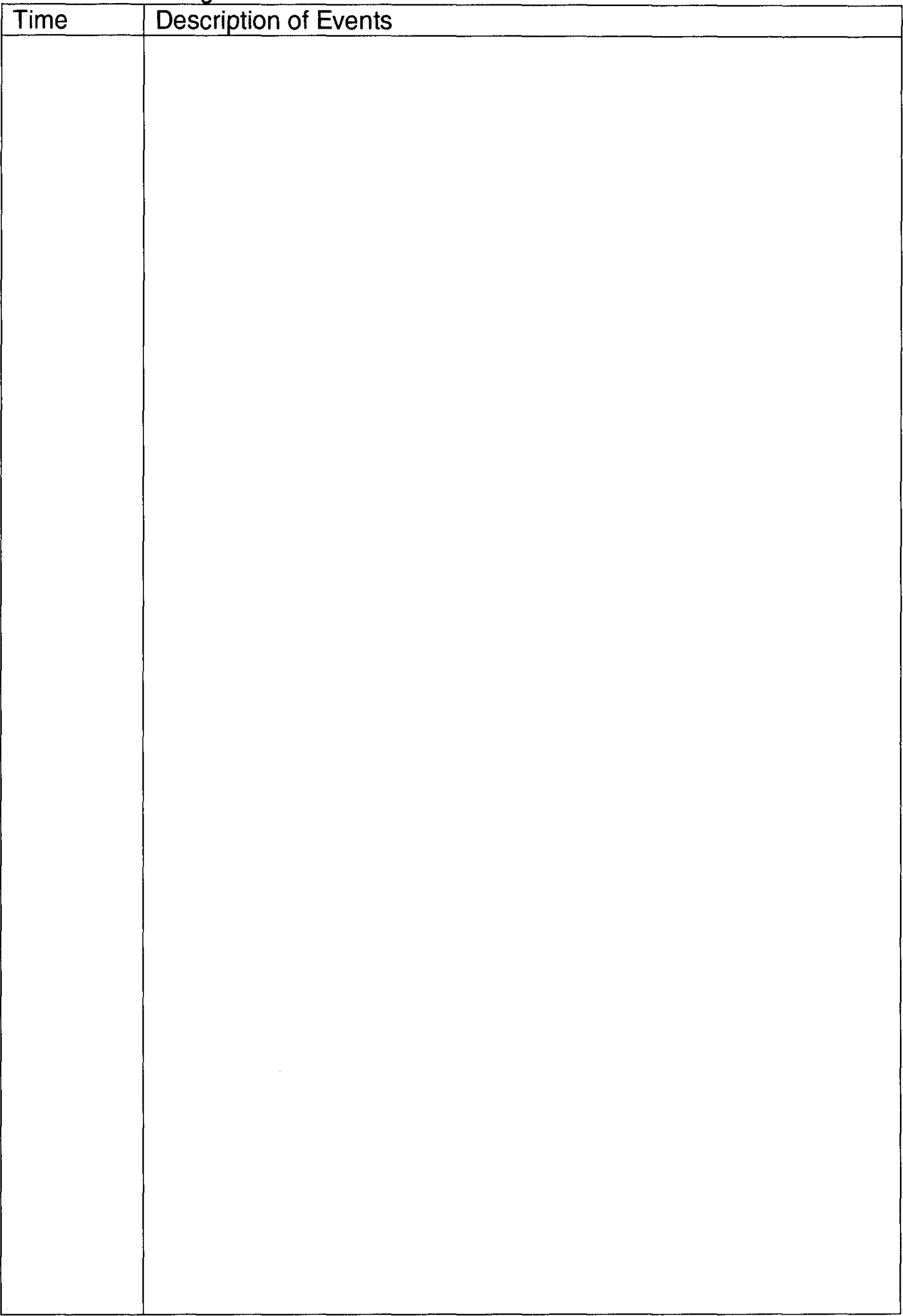


16) 1) The instructional strategies and activities respected students' prior knowledge and the preconceptions inherent therein.

17) 2) The lesson was designed to engage students as members of a learning community.

18) 3) In this lesson, student exploration preceded formal presentation.

19) 4) This lesson encouraged students to seek and value alternative modes of investigation or of problem solving.

20) 5) The focus and direction of the lesson was often determined by ideas originating with students.

\section{CONTENT}

\section{Communicative Interactions}

21) Students were involved in the communication of their ideas to others using a variety of means and media.

22) The teacher's questions triggered divergent modes of thinking.

23) There was a high proportion of student talk and a significant amount of it occurred between and among students.

24) Student questions and comments often determined the focus and direction of classroom discourse.

25) There was a climate of respect for what others had to say.

0

$$
0
$$

0

$\begin{array}{lll}2 & 3 & 4\end{array}$

0

0

$\begin{array}{lllll}0 & 1 & 2 & 3 & 4\end{array}$

$\begin{array}{lllll}0 & 1 & 2 & 3 & 4\end{array}$

\section{Student/Teacher Relationships}

26) Active participation of students was encouraged and valued.

27) Students were encouraged to generate conjectures, alternative solution strategies, and ways of interpreting evidence.

28) In general the teacher was patient with students.

29) The teacher acted as a resource person, working to support and enhance student investigations.

30) The metaphor "teacher as listener" was very characteristic $\begin{array}{llllll}0 & 1 & 2 & 3 & 4\end{array}$ of this classroom.

Additional comments you may wish to make about this lesson. 
Course Information

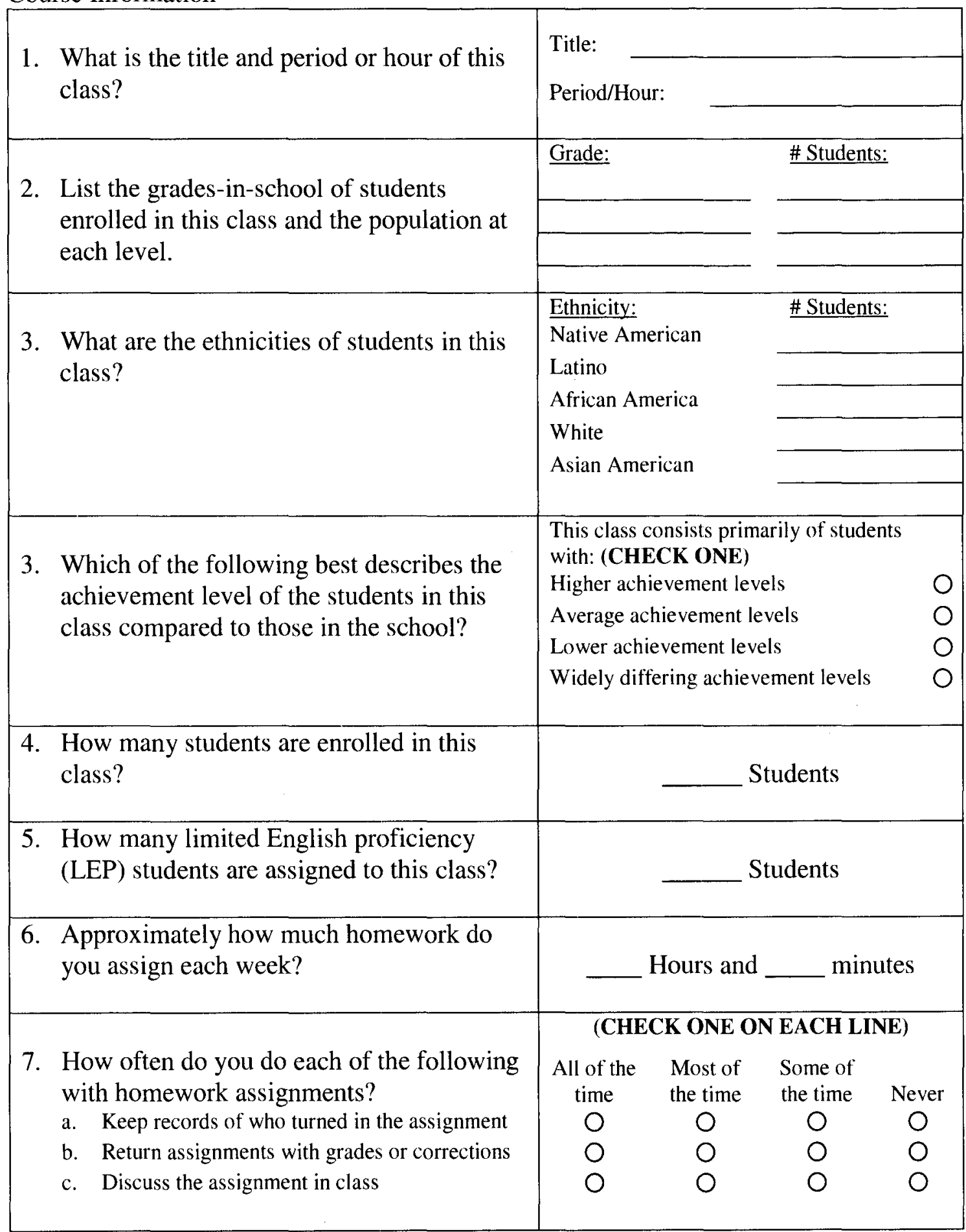


8. What use do you make of the following instructional

\section{(CHECK ONE ON EACH LINE)} materials?

Primary Secondary

resource resource

used used Rarely Not

frequently frequently used used
a. Textbooks
b. Reading materials other than textbooks
c. Audio-visual materials
d. Other

0

O

0

O
0

O

O
0

0

O

0

0

O

O

O

\section{(CHECK ONE)}

9. Approximately what percentage of this textbook/workbook will you cover in this course?

$\begin{array}{lllllll}0- & 50- & 60- & 70- & 80- & 90- & 100\end{array}$ $\begin{array}{lllllll}49 \% & 59 \% & 69 \% & 79 \% & 89 \% & 99 \% & \%\end{array}$

$\begin{array}{lllllll}0 & 0 & 0 & 0 & 0 & 0 & 0\end{array}$

10. Please give your opinion about each of the following statements relating to this textbook/workbook. This textbook:
a. is at a reading level which is too difficult for most of my students.
b. helps develop problem-solving skills.
c. explains concepts clearly.
d. provides good suggestions for homework assignments.
e. does a good job covering the subject area
f. is considered interesting by most students in the class.

11. How adequately prepared do you feel to teach the subject matter covered in this course?

Very well prepared

(CHECK ONE ON EACH LINE)

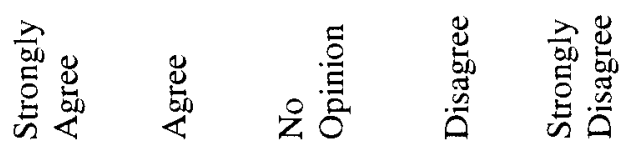

\begin{tabular}{|c|c|c|c|c|c|c|c|c|}
\hline \multicolumn{2}{|r|}{ suoject matter covered in tnis course? } & \multicolumn{7}{|c|}{$\begin{array}{l}\text { Well prepared } \\
\text { Adequately prepared } \\
\text { Somewhat prepared } \\
\text { Totally unprepared }\end{array}$} \\
\hline 12. & $\begin{array}{l}\text { Approximately how many hours per week does this } \\
\text { class meet regularly? }\end{array}$ & \multicolumn{4}{|c|}{ hours lecture } & \multicolumn{3}{|c|}{ hours lab } \\
\hline \multirow{2}{*}{\multicolumn{2}{|c|}{$\begin{array}{l}\text { 3. Indicate about how much time you spend each week } \\
\text { doing each of the following with this class. }\end{array}$}} & \multicolumn{7}{|c|}{ (CHECK ONE ON EACH LINE) } \\
\hline & & None & $<1 \mathrm{hr}$. & $1 \mathrm{hr}$. & $2 \mathrm{hr}$. & $3 \mathrm{hr}$. & & \\
\hline & a. Providing instruction to the class as a whole & & & & & 0 & & $\mathrm{O}$ \\
\hline & $\begin{array}{l}\text { b. Providing instruction to small groups of } \\
\text { students }\end{array}$ & & & & O & O & O & $\mathrm{O}$ \\
\hline & c. Providing instruction to individual students & O & $\mathrm{O}$ & $\mathrm{O}$ & O & 0 & O & 0 \\
\hline & d. Maintaining order/disciplining students & O & $\mathrm{O}$ & 0 & O & 0 & 0 & O \\
\hline & e. Administering tests or quizzes & 0 & & O & 0 & O & O & 0 \\
\hline & $\begin{array}{l}\text { f. Performing routine administrative tasks (e.g., } \\
\text { taking attendance, making announcements, etc.) }\end{array}$ & $\mathrm{O}$ & $\mathrm{O}$ & $\mathrm{O}$ & 0 & $\mathrm{O}$ & O & 0 \\
\hline & g. Conducting lab periods & 0 & 0 & 0 & 0 & 0 & 0 & $\mathrm{O}$ \\
\hline
\end{tabular}


Appendix F To what extent does each statement describe the student...

\begin{tabular}{|c|c|c|c|c|c|c|c|c|c|c|c|c|c|c|c|c|c|c|c|c|c|c|c|c|c|c|c|c|c|c|c|}
\hline \multirow{2}{*}{ 咅 } & \multirow{2}{*}{$\begin{array}{l} \\
\end{array}$} & \multicolumn{3}{|c|}{$\begin{array}{l}\text { Consistently } \\
\text { performs below } \\
\text { ability? }\end{array}$} & \multicolumn{3}{|c|}{$\begin{array}{c}\text { Rarely completes } \\
\text { homework? }\end{array}$} & \multicolumn{3}{|c|}{$\begin{array}{l}\text { Is frequently } \\
\text { absent? }\end{array}$} & \multicolumn{3}{|c|}{$\begin{array}{c}\text { Is frequently } \\
\text { tardy? }\end{array}$} & \multicolumn{3}{|c|}{$\begin{array}{l}\text { Is consistently } \\
\text { inattentive in } \\
\text { class? }\end{array}$} & \multicolumn{3}{|c|}{$\begin{array}{c}\text { Is excessively } \\
\text { passive or } \\
\text { withdrawn? }\end{array}$} & \multicolumn{3}{|c|}{$\begin{array}{c}\text { Is frequently } \\
\text { disruptive? }\end{array}$} & \multicolumn{3}{|c|}{$\begin{array}{l}\text { Has fallen behind } \\
\text { because of poor } \\
\text { health? }\end{array}$} & \multicolumn{3}{|c|}{$\begin{array}{c}\text { Has a handicap } \\
\text { affecting school } \\
\text { work }\end{array}$} & \multicolumn{3}{|c|}{$\begin{array}{l}\text { Is a limited } \\
\text { English } \\
\text { proficiency } \\
\text { student? }\end{array}$} \\
\hline & & $\mathbf{Y}$ & $\mathbf{N}$ & D & $\mathbf{Y}$ & $\mathbf{N}$ & D & $\mathbf{Y}$ & $\mathbf{N}$ & D & $\mathbf{Y}$ & $\mathbf{N}$ & D & $\mathbf{Y}$ & $\mathbf{N}$ & D & $\mathbf{Y}$ & $\mathbf{N}$ & D & $Y$ & $\mathbf{N}$ & D & $\mathbf{Y}$ & $\mathbf{N}$ & $\mathbf{D}$ & $\mathbf{Y}$ & $\mathbf{N}$ & D & $\mathbf{Y}$ & & \\
\hline 001 & & 0 & 0 & 0 & 0 & 0 & 0 & 0 & 0 & 0 & 0 & 0 & 0 & 0 & 0 & 0 & 0 & 0 & 0 & 0 & 0 & 0 & 0 & 0 & 0 & 0 & 0 & 0 & 0 & $C$ & O \\
\hline 002 & & 0 & 0 & 0 & 0 & 0 & 0 & 0 & 0 & 0 & 0 & 0 & 0 & 0 & 0 & 0 & 0 & 0 & 0 & 0 & 0 & 0 & 0 & 0 & 0 & 0 & 0 & 0 & 0 & 0 & 0 \\
\hline 003 & & 0 & 0 & 0 & $\mathrm{O}$ & 0 & 0 & 0 & 0 & 0 & 0 & 0 & 0 & 0 & 0 & 0 & 0 & 0 & 0 & 0 & 0 & 0 & 0 & 0 & 0 & 0 & 0 & 0 & 0 & $c$ & 0 \\
\hline 004 & & 0 & 0 & 0 & 0 & 0 & 0 & 0 & 0 & 0 & 0 & 0 & 0 & 0 & 0 & 0 & 0 & 0 & 0 & 0 & 0 & 0 & 0 & 0 & 0 & 0 & 0 & 0 & 0 & 0 & 0 \\
\hline 005 & & 0 & 0 & 0 & 0 & 0 & 0 & 0 & 0 & 0 & 0 & 0 & 0 & 0 & 0 & 0 & 0 & 0 & 0 & 0 & 0 & 0 & 0 & 0 & 0 & 0 & 0 & 0 & 0 & C & 0 \\
\hline 006 & & 0 & 0 & 0 & 0 & 0 & 0 & 0 & 0 & 0 & 0 & 0 & 0 & 0 & 0 & 0 & 0 & 0 & 0 & 0 & 0 & 0 & 0 & 0 & 0 & 0 & 0 & 0 & 0 & 0 & 0 \\
\hline 007 & & 0 & 0 & 0 & 0 & 0 & 0 & 0 & 0 & 0 & 0 & 0 & 0 & 0 & 0 & 0 & 0 & 0 & 0 & 0 & 0 & 0 & 0 & 0 & 0 & 0 & 0 & 0 & 0 & C & $\mathrm{O}$ \\
\hline 008 & & 0 & 0 & 0 & 0 & 0 & 0 & 0 & 0 & 0 & 0 & 0 & 0 & 0 & 0 & 0 & 0 & 0 & 0 & 0 & 0 & 0 & 0 & 0 & 0 & 0 & 0 & 0 & 0 & 0 & 0 \\
\hline 009 & & 0 & 0 & 0 & 0 & 0 & 0 & 0 & 0 & 0 & 0 & 0 & 0 & 0 & 0 & 0 & 0 & 0 & 0 & 0 & 0 & 0 & 0 & 0 & 0 & 0 & 0 & 0 & 0 & C & 0 \\
\hline 010 & & 0 & 0 & 0 & 0 & 0 & 0 & 0 & 0 & 0 & 0 & 0 & 0 & 0 & 0 & 0 & 0 & 0 & 0 & 0 & 0 & 0 & 0 & 0 & 0 & 0 & 0 & 0 & 0 & 0 & 0 \\
\hline 011 & & 0 & 0 & 0 & 0 & 0 & 0 & 0 & 0 & 0 & 0 & 0 & 0 & 0 & 0 & 0 & 0 & 0 & 0 & 0 & 0 & 0 & 0 & 0 & 0 & 0 & 0 & 0 & 0 & C & 0 \\
\hline 012 & & 0 & 0 & 0 & 0 & 0 & 0 & 0 & 0 & 0 & 0 & 0 & 0 & 0 & 0 & 0 & 0 & 0 & 0 & 0 & 0 & 0 & 0 & 0 & 0 & 0 & 0 & 0 & 0 & 0 & 0 \\
\hline 013 & & 0 & 0 & 0 & 0 & 0 & 0 & 0 & 0 & 0 & 0 & 0 & 0 & 0 & 0 & 0 & 0 & 0 & 0 & 0 & 0 & 0 & 0 & 0 & 0 & 0 & 0 & 0 & 0 & C & 0 \\
\hline 014 & & 0 & 0 & 0 & 0 & 0 & 0 & 0 & 0 & 0 & 0 & 0 & 0 & 0 & 0 & 0 & 0 & 0 & 0 & 0 & 0 & 0 & 0 & 0 & 0 & 0 & 0 & 0 & 0 & 0 & 0 \\
\hline 015 & & 0 & 0 & 0 & 0 & 0 & 0 & 0 & 0 & 0 & 0 & 0 & 0 & 0 & 0 & 0 & 0 & 0 & 0 & 0 & 0 & 0 & 0 & 0 & 0 & 0 & 0 & 0 & 0 & C & 0 \\
\hline 016 & & 0 & 0 & 0 & 0 & 0 & 0 & 0 & 0 & 0 & 0 & 0 & 0 & 0 & 0 & 0 & 0 & 0 & 0 & 0 & 0 & 0 & 0 & 0 & 0 & 0 & 0 & 0 & 0 & 0 & 0 \\
\hline 017 & & 0 & 0 & 0 & 0 & 0 & 0 & 0 & 0 & 0 & 0 & 0 & 0 & 0 & 0 & 0 & 0 & 0 & 0 & 0 & 0 & 0 & 0 & 0 & 0 & 0 & 0 & 0 & 0 & C & 0 \\
\hline 018 & & 0 & 0 & 0 & 0 & 0 & 0 & 0 & 0 & 0 & 0 & 0 & 0 & 0 & 0 & 0 & 0 & 0 & 0 & 0 & 0 & 0 & 0 & 0 & 0 & 0 & 0 & 0 & 0 & 0 & 0 \\
\hline 019 & & 0 & 0 & 0 & 0 & 0 & 0 & 0 & 0 & 0 & 0 & 0 & 0 & 0 & 0 & 0 & 0 & 0 & 0 & 0 & 0 & 0 & 0 & 0 & 0 & 0 & 0 & 0 & 0 & $C$ & 0 \\
\hline 020 & & 0 & 0 & 0 & 0 & 0 & 0 & 0 & 0 & 0 & 0 & 0 & 0 & 0 & 0 & 0 & 0 & 0 & 0 & 0 & 0 & 0 & 0 & 0 & 0 & 0 & 0 & 0 & 0 & 0 & 0 \\
\hline 021 & & 0 & 0 & 0 & 0 & 0 & 0 & 0 & 0 & 0 & 0 & 0 & 0 & 0 & 0 & 0 & 0 & 0 & 0 & 0 & 0 & 0 & 0 & 0 & 0 & 0 & 0 & 0 & 0 & C & 0 \\
\hline 022 & & 0 & 0 & 0 & 0 & 0 & 0 & 0 & 0 & 0 & 0 & 0 & 0 & 0 & 0 & 0 & 0 & 0 & 0 & 0 & 0 & 0 & 0 & 0 & 0 & 0 & 0 & 0 & 0 & 0 & 0 \\
\hline 023 & & 0 & 0 & 0 & 0 & 0 & 0 & 0 & 0 & 0 & 0 & 0 & 0 & 0 & 0 & 0 & 0 & 0 & 0 & 0 & 0 & 0 & & 0 & 0 & & 0 & 0 & 0 & C & 0 \\
\hline 024 & & 0 & 0 & $\mathrm{O}$ & 0 & 0 & 0 & 0 & 0 & 0 & 0 & 0 & 0 & 0 & 0 & 0 & 0 & 0 & 0 & 0 & 0 & 0 & 0 & 0 & 0 & 0 & 0 & 0 & 0 & 0 & 0 \\
\hline 025 & & 0 & 0 & 0 & 0 & 0 & 0 & 0 & 0 & 0 & 0 & 0 & 0 & 0 & 0 & 0 & 0 & 0 & 0 & 0 & 0 & 0 & 0 & 0 & 0 & 0 & 0 & 0 & 0 & C & 0 \\
\hline 026 & & 0 & 0 & 0 & 0 & 0 & 0 & 0 & 0 & 0 & 0 & 0 & 0 & 0 & 0 & 0 & 0 & 0 & 0 & 0 & 0 & 0 & 0 & 0 & 0 & 0 & 0 & 0 & 0 & 0 & 0 \\
\hline 027 & & 0 & 0 & 0 & 0 & 0 & 0 & 0 & 0 & 0 & 0 & 0 & 0 & 0 & 0 & 0 & 0 & 0 & 0 & 0 & 0 & 0 & 0 & 0 & 0 & 0 & 0 & 0 & 0 & C & 0 \\
\hline 028 & & 0 & 0 & 0 & 0 & 0 & 0 & 0 & 0 & 0 & 0 & 0 & 0 & 0 & 0 & 0 & 0 & 0 & 0 & 0 & 0 & 0 & 0 & 0 & 0 & 0 & 0 & 0 & 0 & 0 & 0 \\
\hline 029 & & 0 & 0 & 0 & 0 & 0 & 0 & 0 & 0 & 0 & 0 & 0 & 0 & 0 & 0 & 0 & 0 & 0 & 0 & 0 & 0 & 0 & 0 & 0 & 0 & 0 & 0 & 0 & 0 & C & 0 \\
\hline 030 & & 0 & 0 & 0 & 0 & 0 & 0 & 0 & 0 & 0 & 0 & 0 & 0 & 0 & 0 & 0 & 0 & 0 & 0 & 0 & 0 & 0 & 0 & 0 & 0 & 0 & 0 & 0 & 0 & 0 & 0 \\
\hline 031 & & 0 & 0 & 0 & 0 & 0 & 0 & 0 & 0 & 0 & 0 & 0 & 0 & 0 & 0 & 0 & 0 & 0 & 0 & 0 & 0 & 0 & 0 & 0 & 0 & 0 & 0 & 0 & 0 & C & 0 \\
\hline 032 & & 0 & 0 & 0 & 0 & 0 & 0 & 0 & 0 & 0 & 0 & 0 & 0 & 0 & 0 & 0 & 0 & 0 & 0 & 0 & 0 & 0 & 0 & 0 & 0 & 0 & 0 & 0 & 0 & 0 & 0 \\
\hline 033 & & 0 & 0 & 0 & 0 & 0 & 0 & 0 & 0 & 0 & 0 & 0 & 0 & 0 & 0 & 0 & 0 & 0 & 0 & 0 & 0 & 0 & 0 & 0 & 0 & 0 & 0 & 0 & 0 & C & 0 \\
\hline 034 & & 0 & 0 & 0 & 0 & 0 & 0 & 0 & 0 & 0 & 0 & 0 & 0 & 0 & 0 & 0 & 0 & 0 & 0 & 0 & 0 & 0 & 0 & 0 & 0 & 0 & 0 & 0 & 0 & 0 & 0 \\
\hline 035 & & 0 & 0 & 0 & 0 & 0 & 0 & 0 & 0 & 0 & 0 & 0 & 0 & 0 & 0 & 0 & 0 & 0 & 0 & 0 & 0 & 0 & 0 & 0 & 0 & 0 & 0 & 0 & 0 & C & 0 \\
\hline
\end{tabular}


Appendix G1 


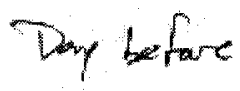

\section{MIAMNCLAB}

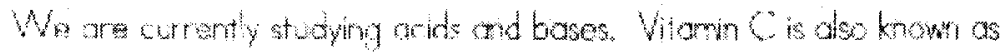

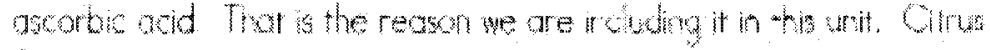

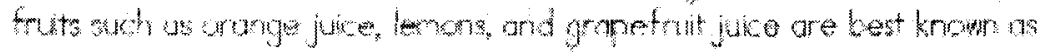
sources of osotbir and Nony other fruts and weythbles are do soreses of ancorto aid

Lwing cels we vitarins by hang them inte molecules that combine with encymes (corohsts) thot untrol terrical reactions in the body. Without

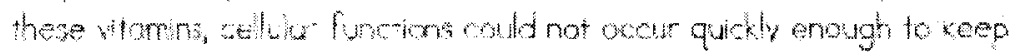
cols dive.

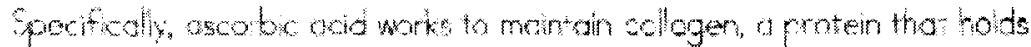
things together in the skin. ligurnewts, and bures. I is krown as port of

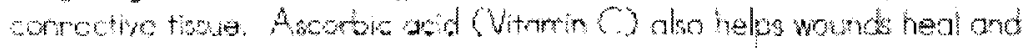
growth of instes, it allows red blood cels to form prevers tenomthaging. and helps font boctenal ntertions.

Bercuse your body doeg not moko or store uxrobic acd for long time perods. is s necessary that it be toden in "reguenty. The procasing. worng and mungement of food products affect the qualy of aromis

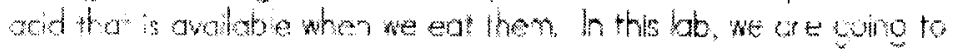

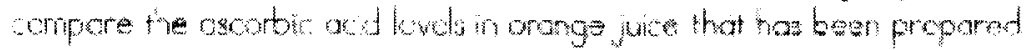
$n$ different ways

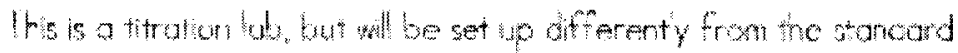

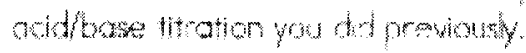

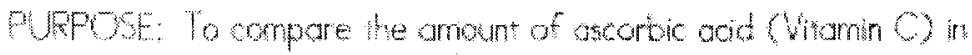
wrious preparat ons of orange juice products by ung smote tiration with icdineistarch salution 


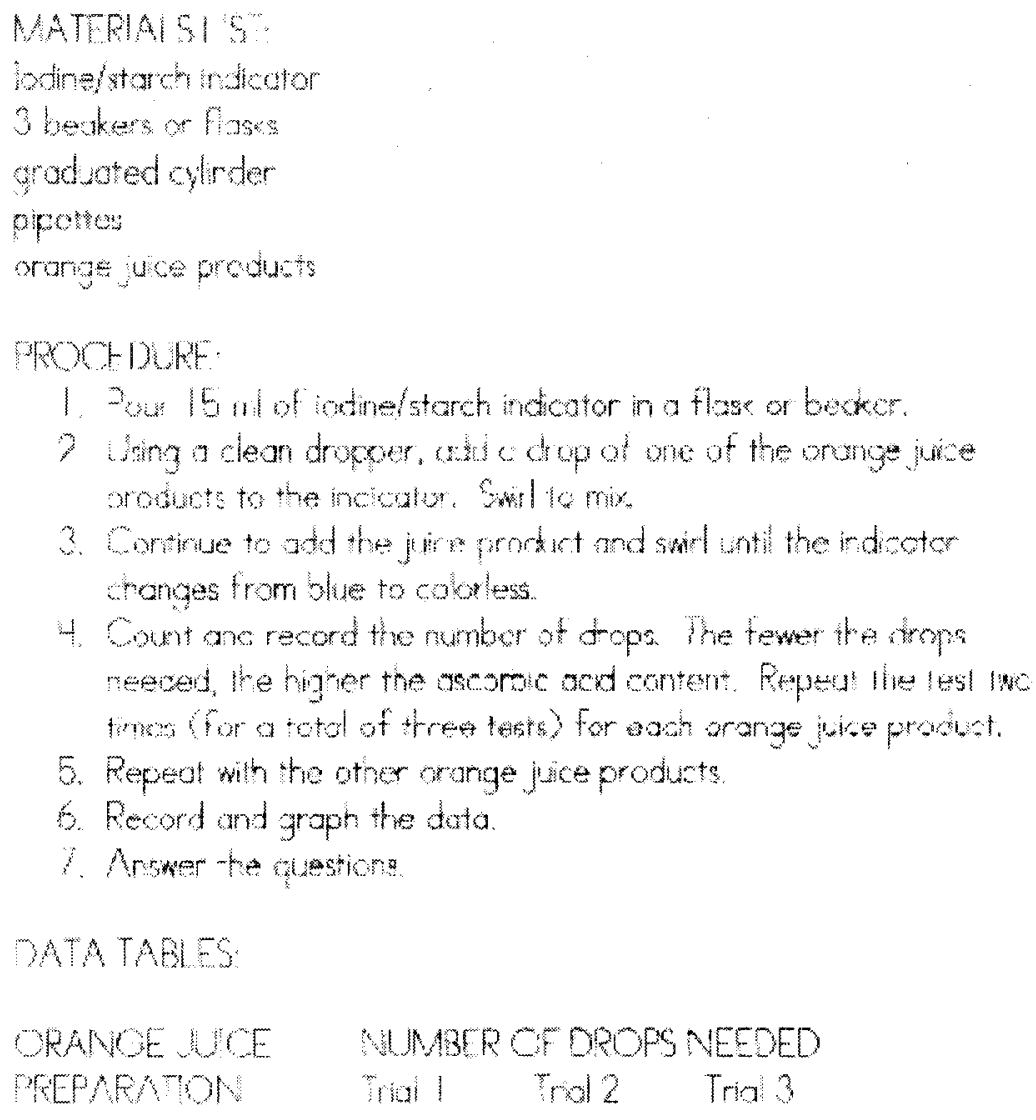


WAWMYLAB

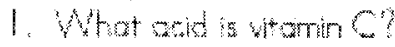

2. Lis 5 uses of womin C an body.

3. What conge juc preporotion hod the grectest ancunt of ascorbic ocid?

4 Mhat ald you do to extablish contros in this experment?

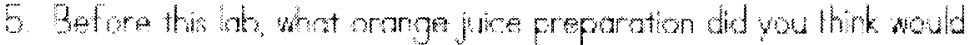
bethe bust ward o soure?

6 What are sore of the foctors that could have decreased the

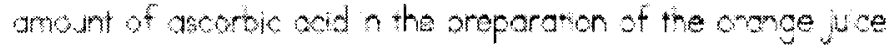
product?

7 How do you krow that he different orenge juines dd not contain

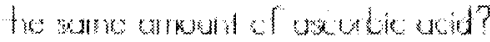

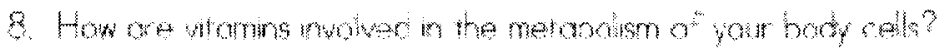

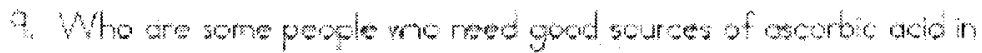
teir diet? 


\section{RUERC FOR ASCORBIC ACID PRO ECT}

1. A rimim of two ond preferbly thee products will be rested

2. Curcet potocol is followed in the prosedur

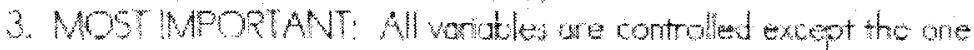
being tesied.

4. Writing is of port on qudity in the folowity format:

a. litroduction: State the purpote and any normeticat the rexts will reed to understord why you cre doing the ksperimert.

b. Expermentel Procadure. Clear sep-by step decripicn of haw to do the expormert. Soneone stould be ablo to cone in and do it from your instructions.

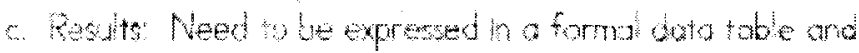
with a bor goph.

d Liscussion: Why do these resuls monter? To whom do hey matter? Reflect on the resilts. Is at whot you expenter to have hoppon?

Aithough you and yor partne- will be using the sume set of dota, roke

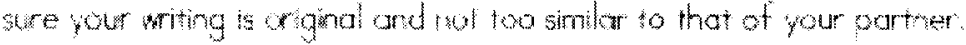


Appendix G2 


\section{Observation and Experiment Introduction to the Scientific Method}

\section{Introduction}

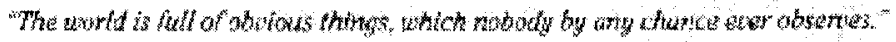

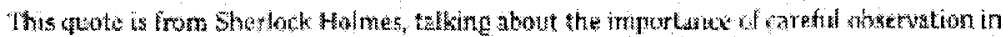

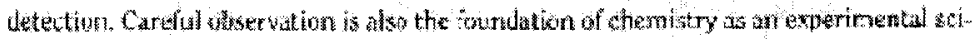

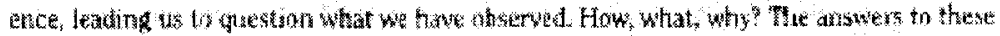

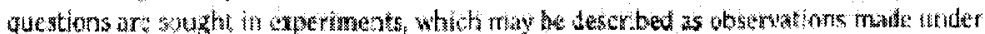

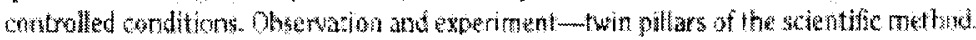

\section{Concepts}

$$
\begin{aligned}
& \text { - Sciendid methot . * Chemistry } \\
& \text { - Tharadist Foneriment }
\end{aligned}
$$

\section{Background}

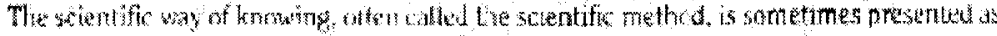

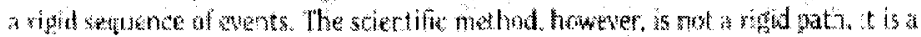

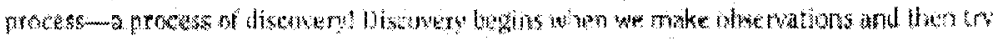

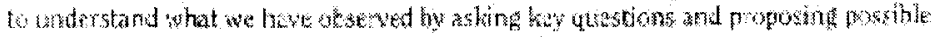

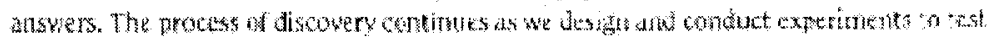

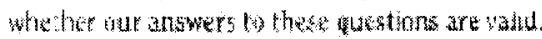

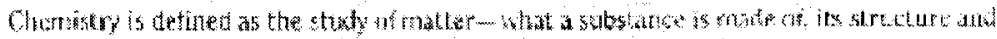

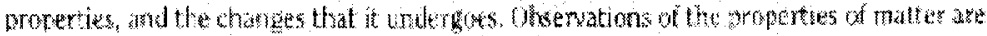

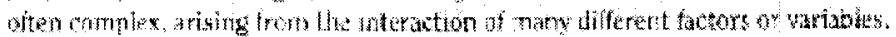

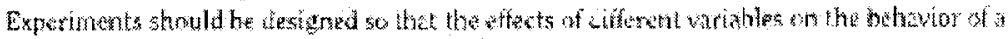

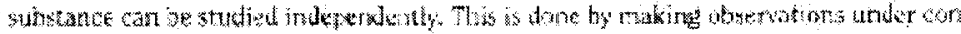

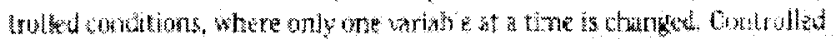

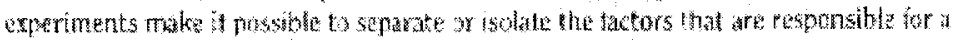

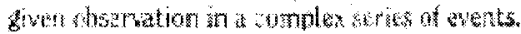

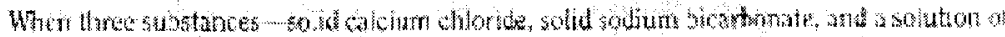

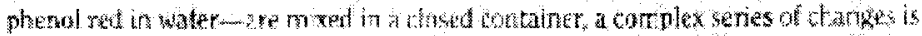

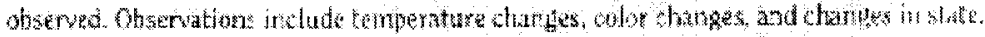

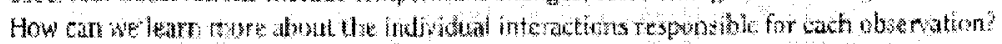

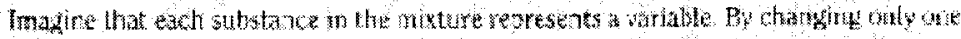

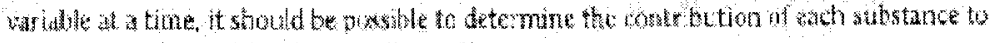

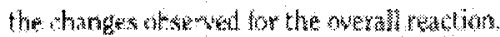




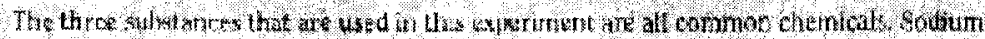

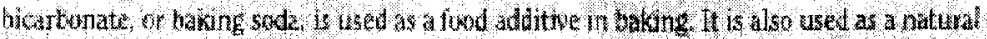

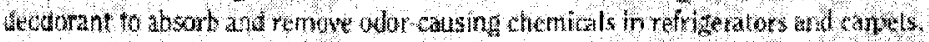

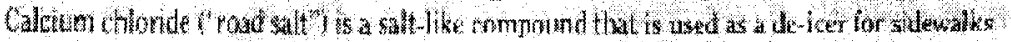

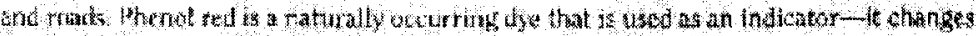
bolor under different conditions.

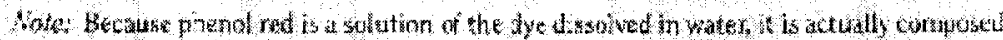

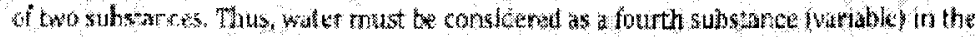
werall reurtion, and its afect should atso be wamind.

\section{Experiment Orerviow}

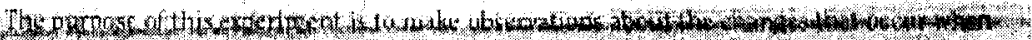

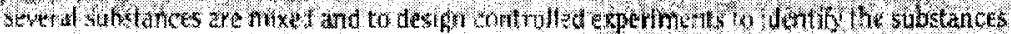

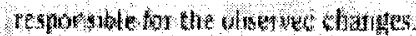

\section{Pre-Lab Questions}

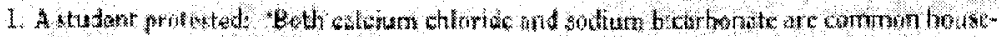

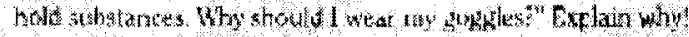

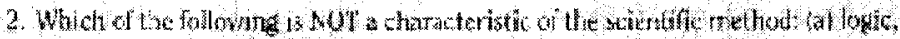

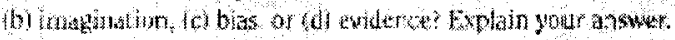

\section{Materials}

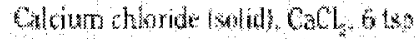

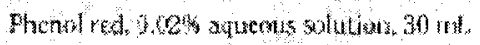

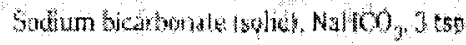

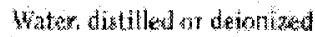

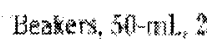

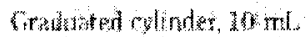

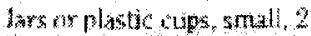

Masuring woons, tatanow and half $r$ easoon, 1 ad

Hentorlaberne

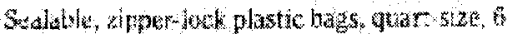

Whar torthe

\section{Safary Precaution:}

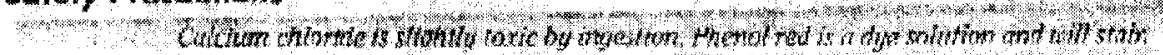

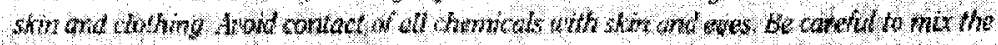

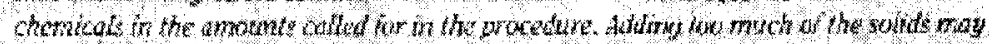

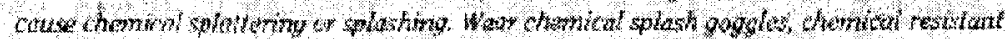

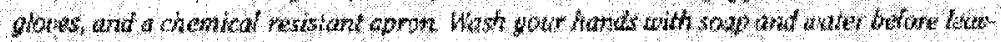
isut the daboratory.

\section{Procodure}

\section{Part A. The Overall Rnoction}

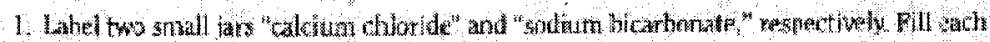

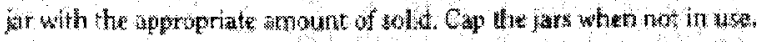




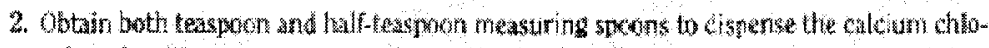

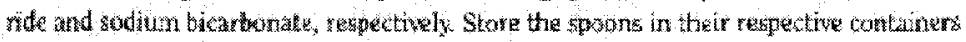

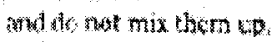

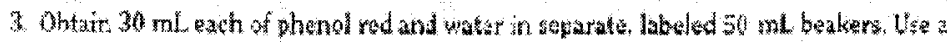

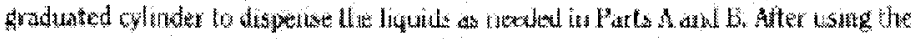

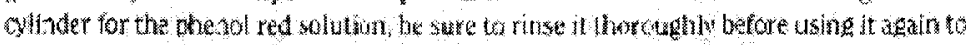
distense distlled water.

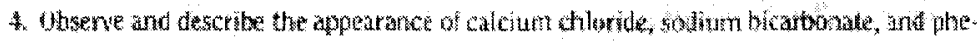
nol red solution, Record the whsewations in Data Table

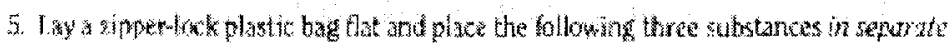
Jaturions in the bate

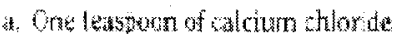

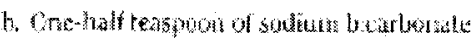

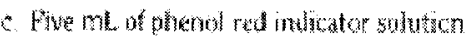

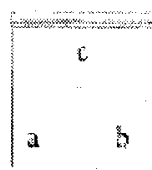

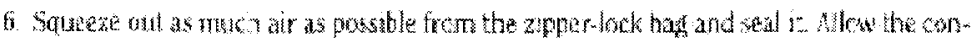
kents wath thorath

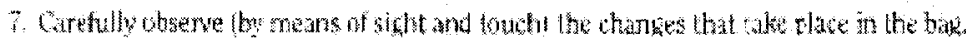

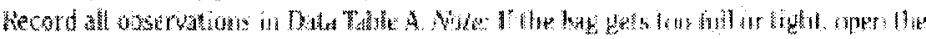

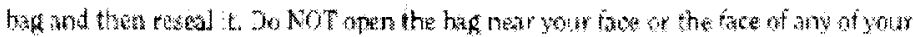
Lobrates.

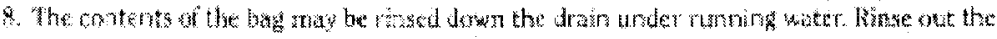

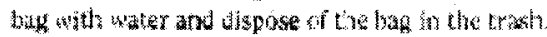

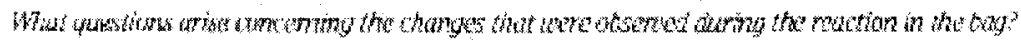

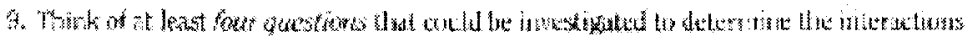

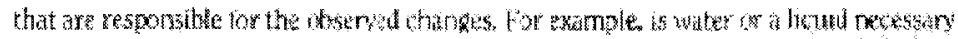

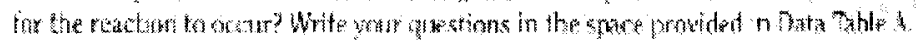

\section{Part B. Controtled Experimants}

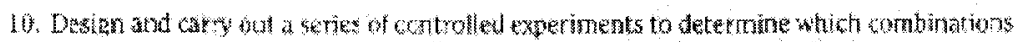

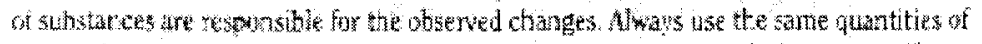

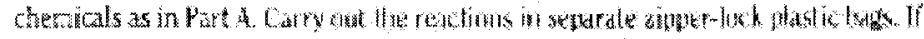

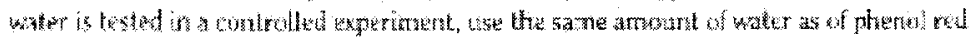
scltion in Fart

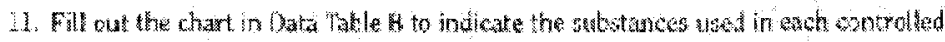

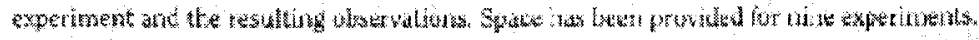

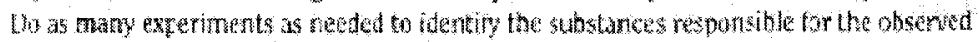

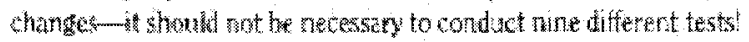


Nome:

Cragktab pribl:

\section{Observation and Experiment}

\section{Dato Table A. The Overell Rocction}

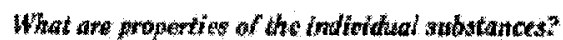

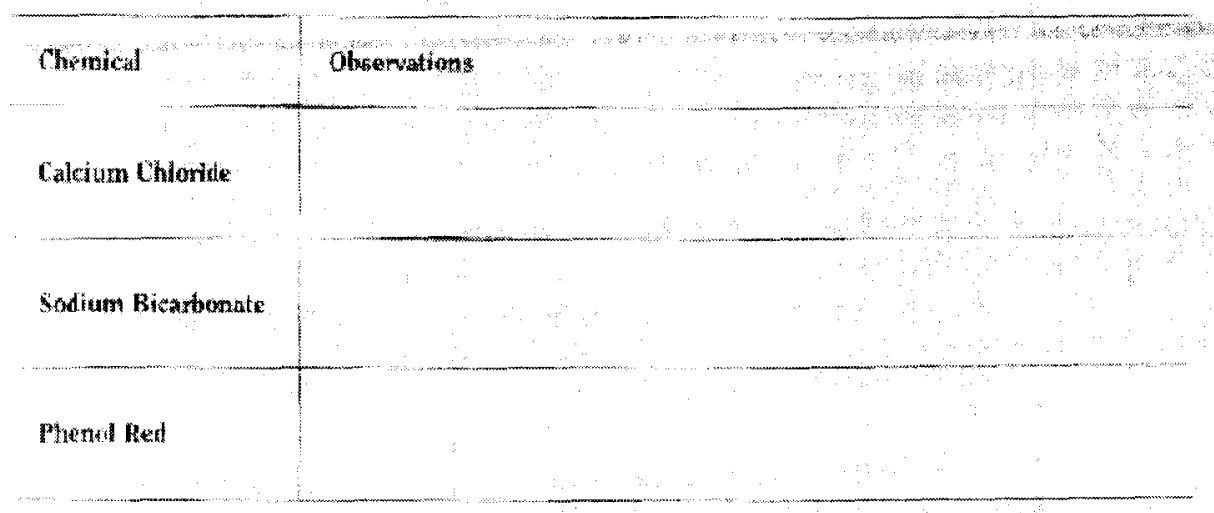

What hoppents when the substancer are mured togother?

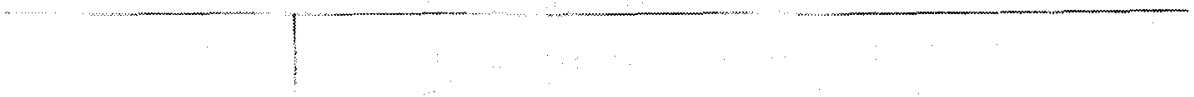

OAveratons

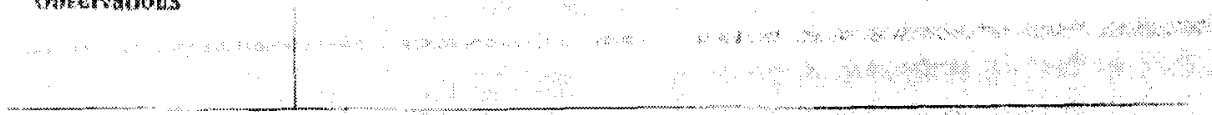

Write four guastions concemong the chinges that were observed

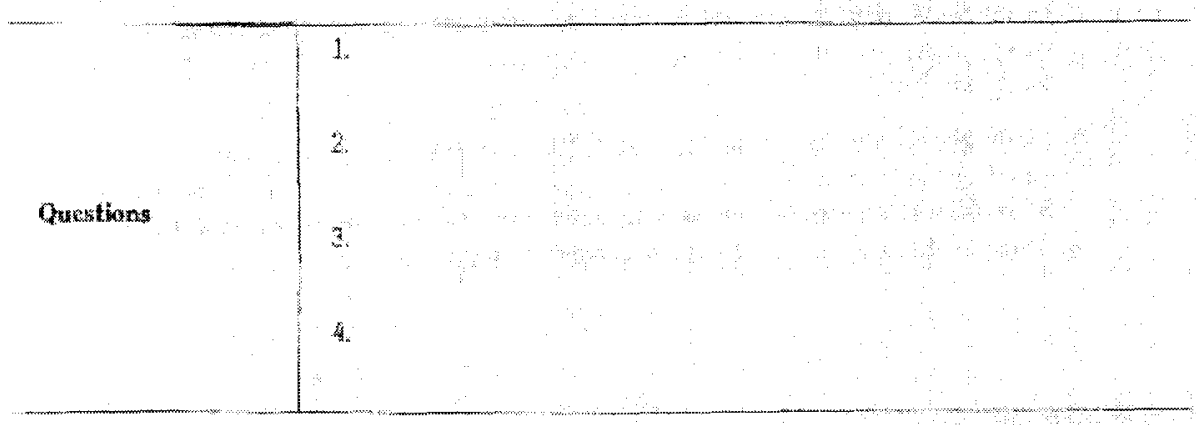

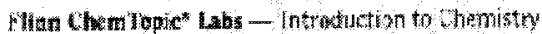


Data Table B. Controlled Experiments

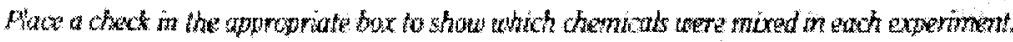

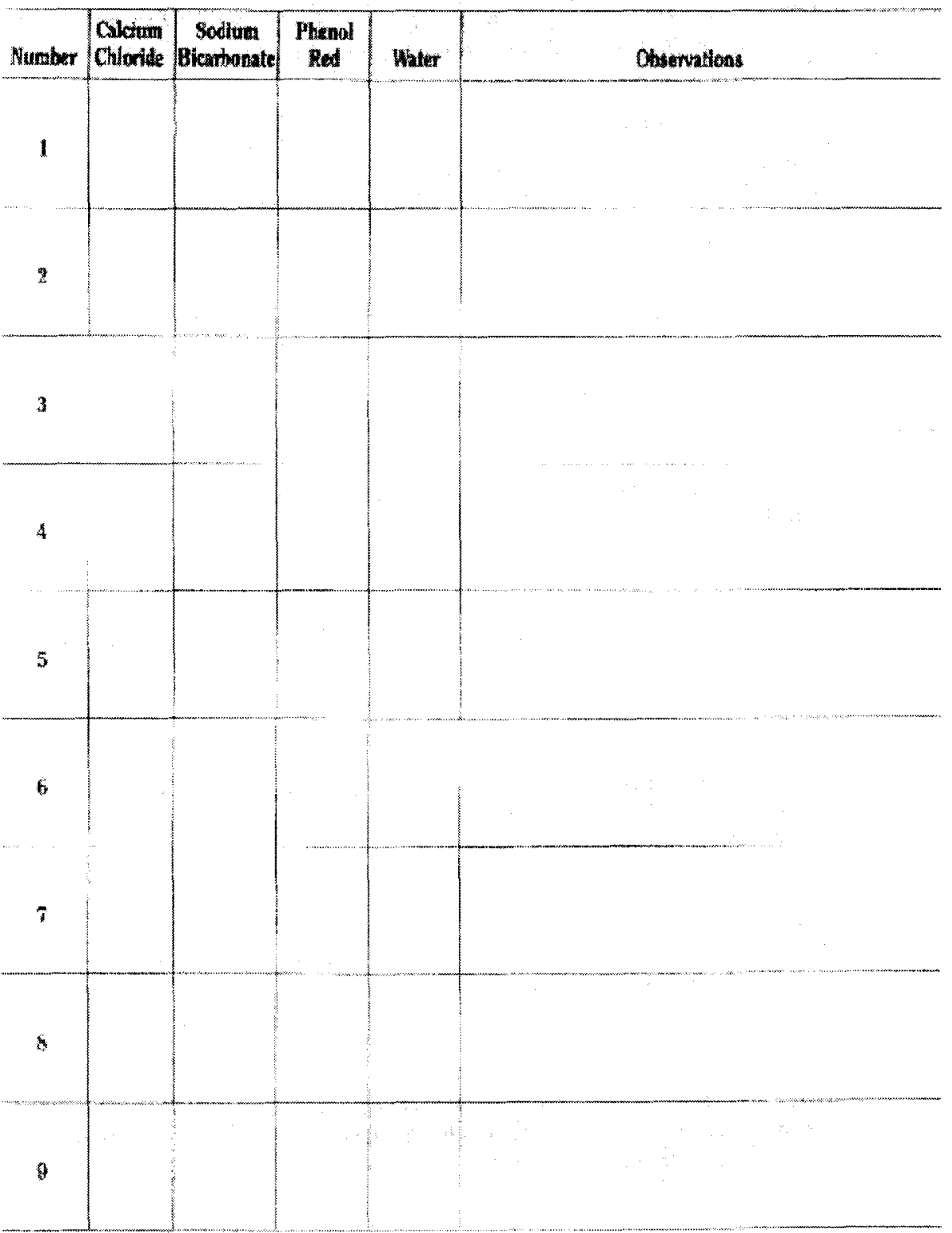

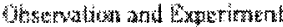




\section{Post-Lab Questions}

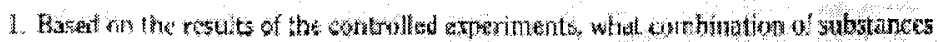

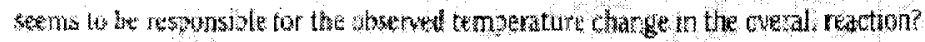

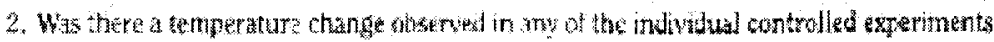

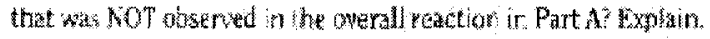

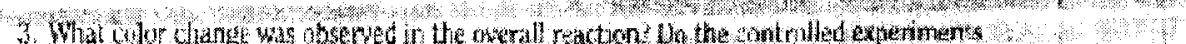

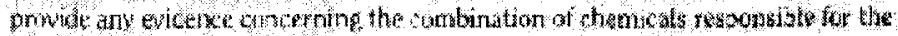
observed color dhasug?

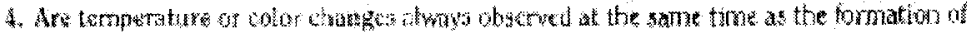
Rastubler? Explain.

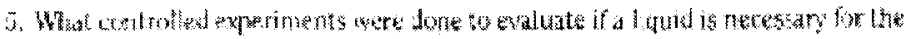

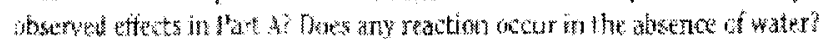

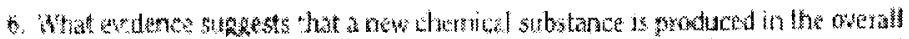

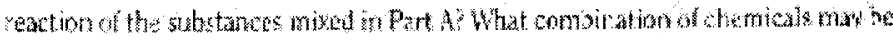

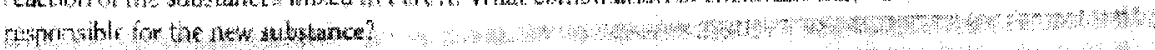

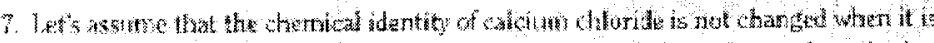

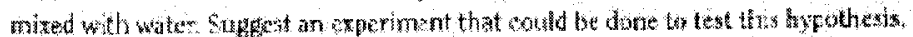

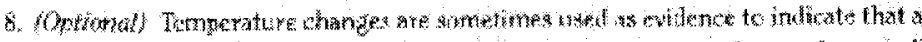

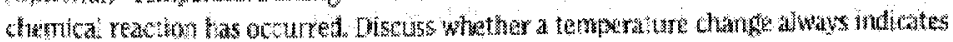

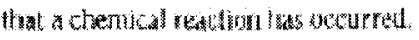

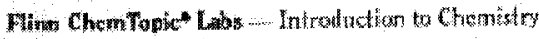


Appendix G3 
WARNING

- This ser omine chentod

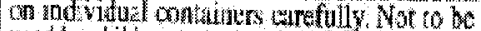

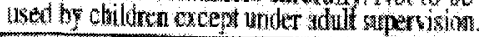

Name

Pention Dute

\section{MYSTERY-MIXTURE ANALYSIS}

\section{Materials}

1 Set of trine whte substanos

1 Vial of mystery mixture

2 Dropper hother of water

10. Minispoons, groen

2 Well trays

- Protective cyewear

\section{Challenge}

Find out shith wo stbstances are in the mystery mixture.

\section{Procedure}

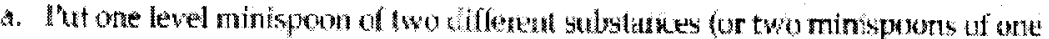
stibstance in a well. Nothe number of the well.

t. Add lodrops of water. Coserve and reord.

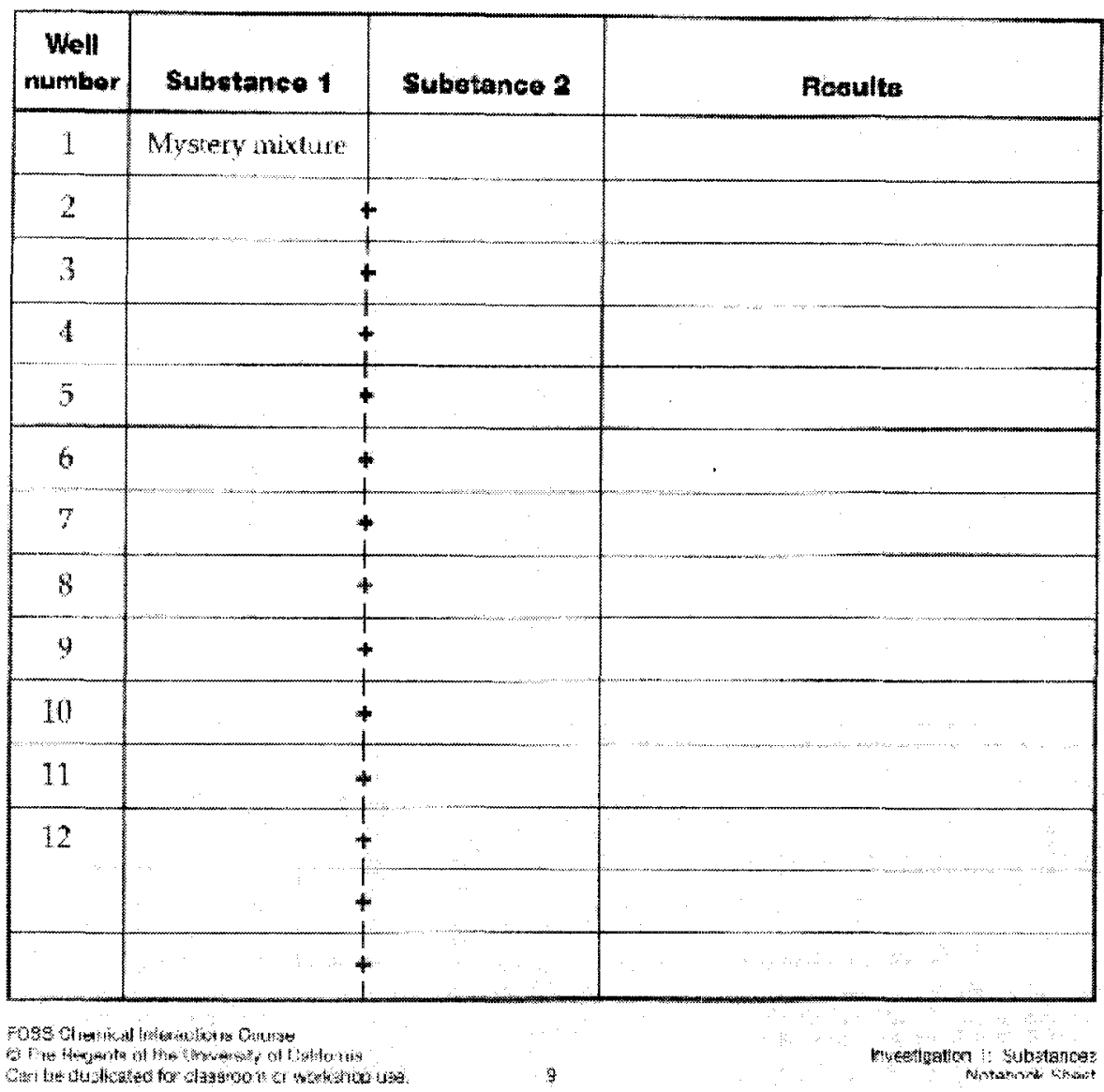



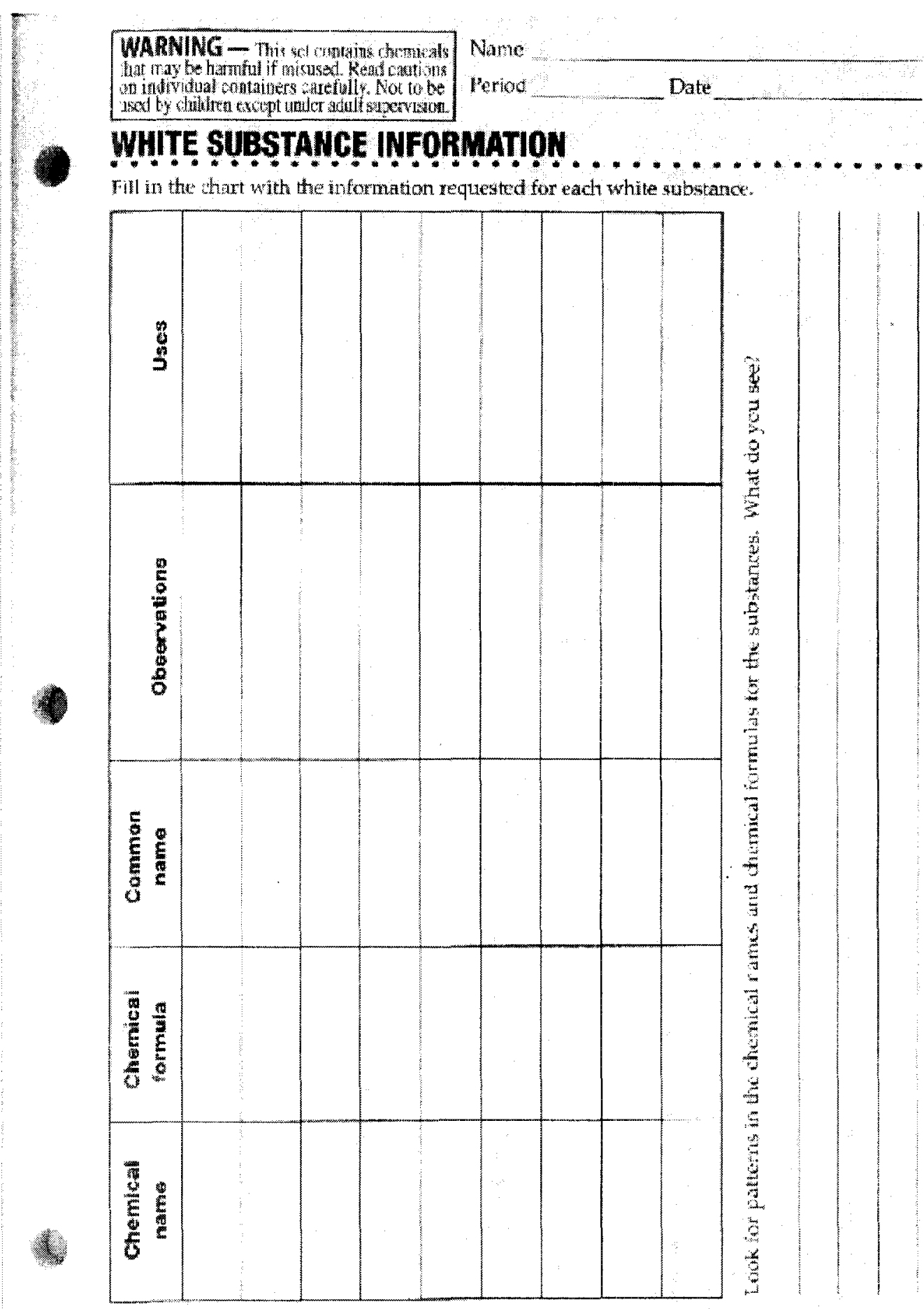

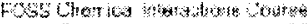

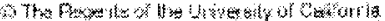

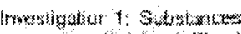

ras 34 -

7 Hothorestom? 


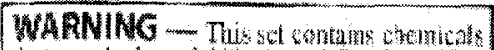

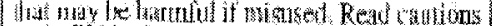

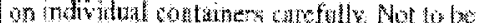

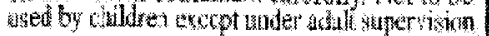

Nante

Period

Dits:

\section{MYSTERY-MIXTURE SUMMARY}

\begin{tabular}{|c|c|c|c|c|}
\hline Well & Shatanos & $\begin{array}{c}\text { Deseription } \\
\text { ol tixing }\end{array}$ & $\begin{array}{c}\text { Other } \\
\text { observations }\end{array}$ & $\begin{array}{l}\text { Large scalo } \\
\text { reactions }\end{array}$ \\
\hline 1 & 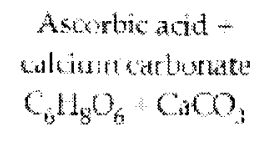 & & & . \\
\hline 2 & 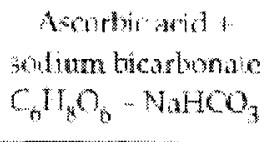 & & & \\
\hline 3 & 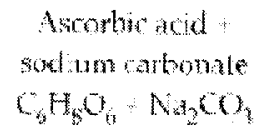 & & & \\
\hline 4 & 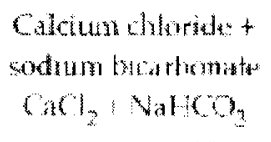 & & & \\
\hline 5 & $\begin{array}{l}\text { Curic acd } \\
\text { alcum carbonate } \\
\mathrm{CHO}_{3}+\mathrm{C}_{3} \mathrm{CO}_{3}\end{array}$ & & & \\
\hline 6 & 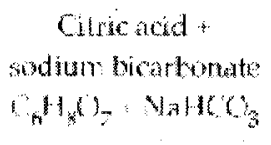 & & & \\
\hline 7 & $\begin{array}{c}\text { Cirricacid } \\
\text { sodum carlunate } \\
\mathrm{CH}_{3} \mathrm{O}_{3} \mid \mathrm{Na}_{2} \mathrm{CO}_{3}\end{array}$ & & & \\
\hline 8 & Mystery mixtuke & & & \\
\hline
\end{tabular}

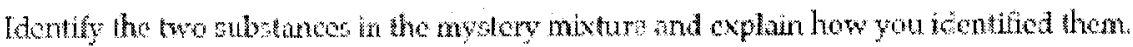

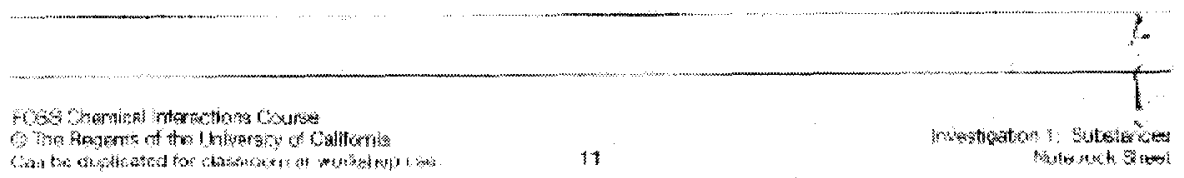


Appendix G4 
Teacher Name: Mr Lagdon Student Nanes:

\section{Smilev Face Family}

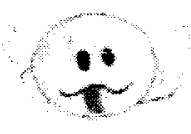

\begin{tabular}{|c|c|c|c|c|}
\hline CATEGORY & ( (2) points) & 3 (15 poituts: & 2 (10 poins) & 1 (sputsis) \\
\hline $\begin{array}{l}\text { Required } \\
\text { Elements }\end{array}$ & $\begin{array}{l}\text { The poster includes all } \\
\text { required elements. Fambly } \\
\text { members } 6 \text { aces, family } \\
\text { members genotypes and } \\
\text { phenotyoes. Punnett } \\
\text { Squares }\end{array}$ & $\begin{array}{l}\text { Pcster is missing one of } \\
\text { the recuired elements }\end{array}$ & $\begin{array}{l}\text { Poster is missing } 20^{*} \text { the } \\
\text { required elements }\end{array}$ & $\begin{array}{l}\text { Poster lacks } 3 \text { or more } \\
\text { required elements. }\end{array}$ \\
\hline Labels & $\begin{array}{l}\text { All items of importante } \\
\text { on the poster are cleary } \\
\text { labeled wth labels that } \\
\text { can be read from at least } \\
3 \text { f away. }\end{array}$ & $\begin{array}{l}\text { Almost all tems of } \\
\text { importance on the poster } \\
\text { are clearty labeled with } \\
\text { labels that can be reac } \\
\text { trom at least } 3 \mathrm{ft} \text { awey. }\end{array}$ & $\begin{array}{l}\text { Many tems of } \\
\text { mportance on the poster } \\
\text { are clearty labeled with } \\
\text { labels that can be read } \\
\text { from al least } 3 \text { t t away. }\end{array}$ & $\begin{array}{l}\text { Lebelis are too small to } \\
\text { view OR no important } \\
\text { items were labeled. }\end{array}$ \\
\hline Accuracy & $\begin{array}{c}\text { All science content } \\
\text { infomation is complete } \\
\text { and accurate }\end{array}$ & $\begin{array}{l}\text { 90\% of all science } \\
\text { content infometion is } \\
\text { complete and accurate }\end{array}$ & $\begin{array}{l}80 \% \text { of all science } \\
\text { content information is } \\
\text { complete and ascurate }\end{array}$ & $\begin{array}{l}70 \% \text { or }{ }^{2} \text { os of all science } \\
\text { content infomation is } \\
\text { complete and accurate }\end{array}$ \\
\hline Attractiveness & $\begin{array}{l}\text { The poster is } \\
\text { exceptionally attractive in } \\
\text { terns of design, layout } \\
\text { and neatneas. }\end{array}$ & $\begin{array}{l}\text { The poster is attractive in } \\
\text { terms ct design, leyout } \\
\text { and neatness. }\end{array}$ & $\begin{array}{l}\text { The poster is acceptably } \\
\text { attractive though it may } \\
\text { be a bit messy. }\end{array}$ & $\begin{array}{l}\text { The poster is distractingly } \\
\text { messy or very poorly } \\
\text { designed. It is not } \\
\text { attractive. }\end{array}$ \\
\hline Grammar & $\begin{array}{l}\text { Thare are no } \\
\text { grammaticalmechanical } \\
\text { mistakes on the poster. }\end{array}$ & $\begin{array}{l}\text { There are } 1-2 \\
\text { grammaticalmechanical } \\
\text { nistakes on the poster }\end{array}$ & $\begin{array}{l}\text { There are } 34 \\
\text { grammaticalmechanical } \\
\text { mistakes on the poste: }\end{array}$ & $\begin{array}{l}\text { I here are more than } 4 \\
\text { grammaticalmethancal } \\
\text { mistakes on he poster. }\end{array}$ \\
\hline
\end{tabular}


Genetics with a Smile

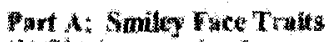

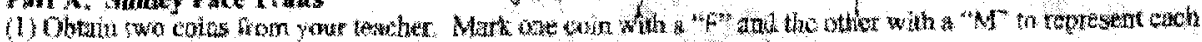

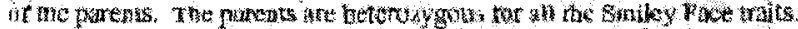

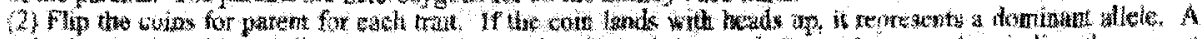

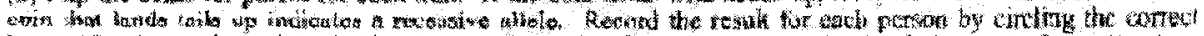

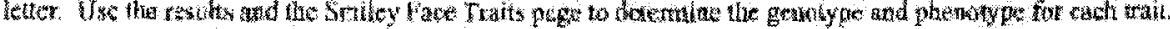

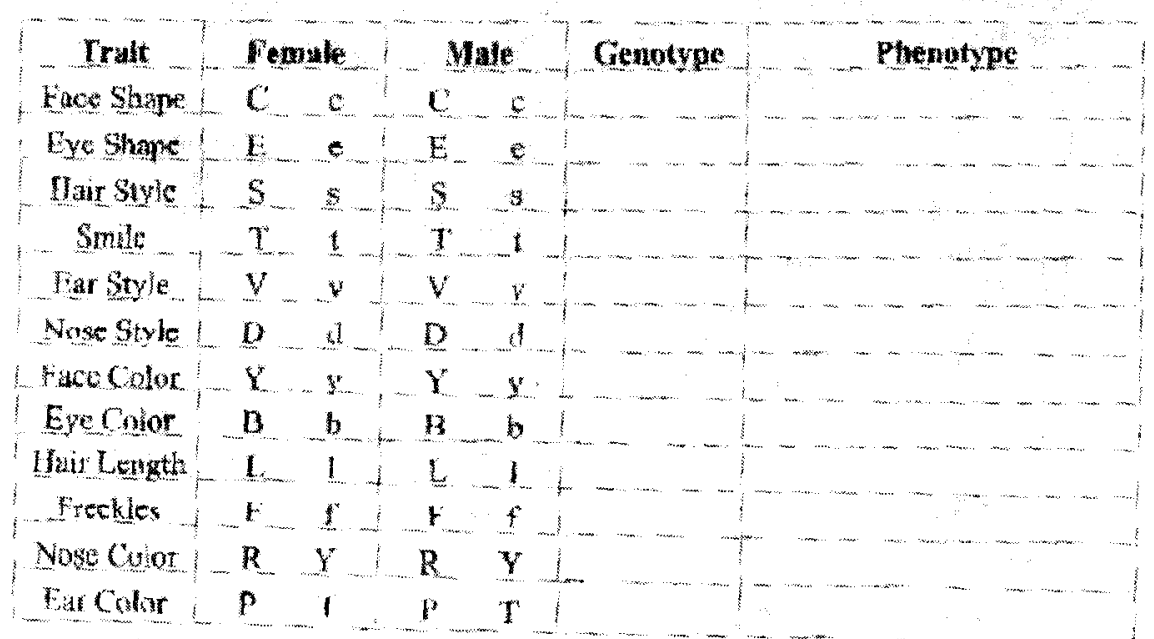

Fart A: Is it a hoy or piw?

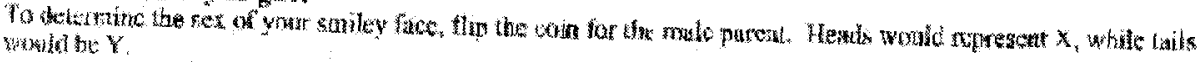

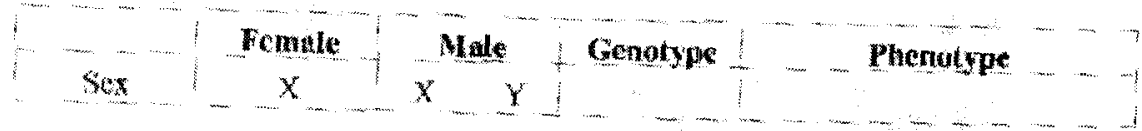

Part C: Crata Yuar Smilky Iowe?

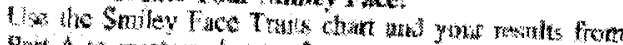

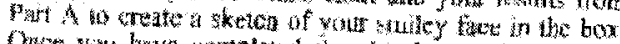

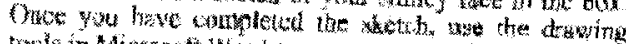

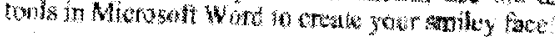

Twe thiags to reminthor

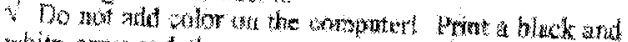

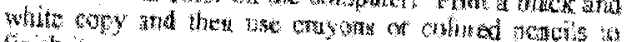
tisis it.

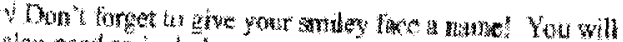

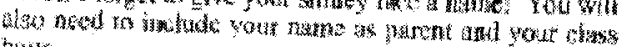
hwer:

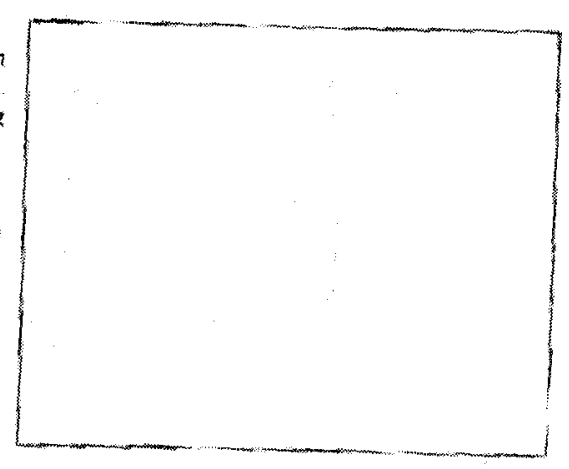

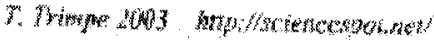




\section{Smiley Fuce Traits}

Face Shnpe

Circle (C)<smiles>C1CCCCCCCCCCC1</smiles>

Eye Stape Star (E)<smiles>C1CCCCCCCCCC1</smiles>

Hair Style

Siraight (S)

$\| 111$

Sinile

Thick (T)

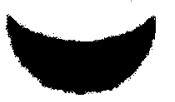

Ear Style

Curved (V)

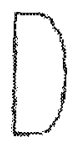

Thin (t)

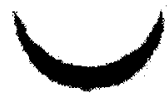

Pointed (w)

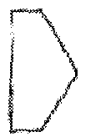

Nose Style

Down (D)

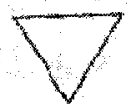

Face Color

Yellow $(Y)$

Oreen (y)

Fye Colo

Blue (B)

Red (b) $\begin{array}{ll}\text { Hair Luengh } & \text { Freekles } \\ \text { Long (L) } & \text { Present (F) } \\ \text { Short (l) } & \text { Absent (f) }\end{array}$

Nose Calor Ear Culor

Red (RR) Hot Pink (PP)

Orange (RY) Purple (PT)

Yellow (YY) Trai (T)

Sex

In determine the sex, ho lip the win for the male parrentio likuds kquals $\mathrm{X}$ and taits equally.

$X X$ - Fernale - Add pink tuw in huir $X Y$ - Male - Add blue bow in hair 
Appendix G5 
LREGE BUILONO PROTITT

IRIDGE CONSTRUCIION RULES

1. ALI BRDGES MUST HH WILT WTIT ONLY THE BAL.SA WOOD SUPPLIED IN YOLIR KIT

2. YOU MAY USE ANY TYPE OF GLUE YOU WISH ALL GLUE IS TO BE FLRNISHED EY YOUR TLAM JOINTS MAY NOT SHOW EXCFSSIVE GLUE-THAT IS, HHEY MAY NOT FE WRAPPED N GLUL NO FEOS OR PINS OF ANY TYPE MAY BE USED NO PAINT IS AL LOWED.

3, YOU MUST MARK THE CENTER POINT AND OUARTEK.POINTS OF YOUR BRDGE WHEN CONSTRUC TION IS FINISHED WITH THE BLACK MARKER PROVIDED IN CLASS

A ANY CROSS-SECTIONAL AREA MAY NOT EXCEED A COMBINED

MEASUREMFNT OF $1 / 2^{\circ}$ AT ANY ONE JOINING OF WOOB FY GLLE BRIDGE SPECIFICATTONS

YOUR BRIDGE MUST BF COMPI ETELY ASSEMBI ED WHEN SUIMTTIED FOR TTSTING IN AIODTTON IT MUST MEET THF FOLLOWLV SPECIFICATIONS

LISIED BELOW.

1. THE BKTOGE MUST BE $18^{\text {" LONO }}$

2. NOT TMLER THAN 6 ,

3. NOT WIDER THAN A",

4. THE FLOOR DOES NOT NFTE TO BE SOLID.

5. MUST DE IN THE IRLSS UESION, BUT DOES NOT HAVE TO FOLLOW ANY TWISS DESIGN PITTERNS.

TTME LNE

WEEK 1 . HTISH A MEASCRED PL.AN WTIH A TOP STDE, AND FRONT VILWS BY THE END OF THE WREK SHOW HOW MANY RCHES WTL BE NTEDED, L.AEEL YOUR DRAWTMS AT EACH SECTIUN UR PART BY TRCHES.

WEEK 2 AND 3 . FUTD THE RRTDCE

WEEK 4 TEST THE BRIDGE 


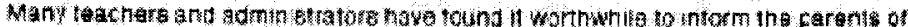

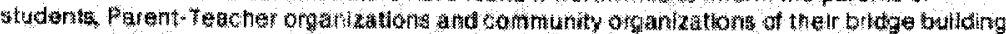

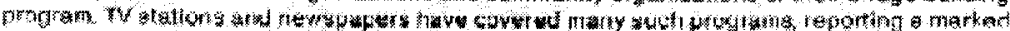

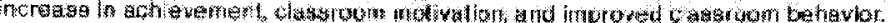

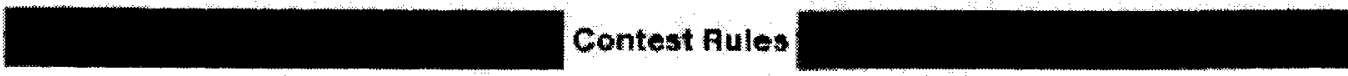

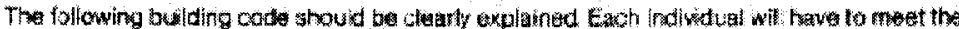
blowing jot soctication 10 sampet in she contes:

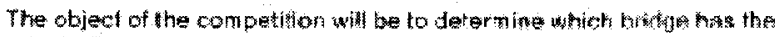
greatest EFFCIENCYY

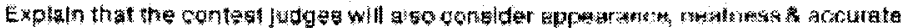

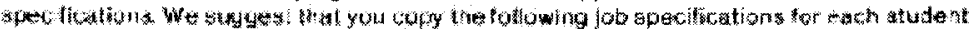

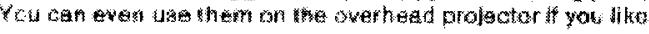

\section{Job Specifleatton}

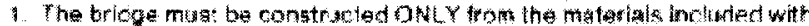

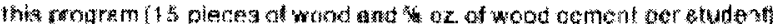
NO OTHEA MATEAIALS Trar be USEd

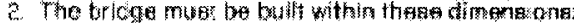

\begin{tabular}{|c|c|c|c|}
\hline & Mridate & Manditued & 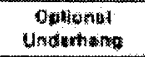 \\
\hline LGiglls & 200 ta 9190 stim & 200 a $300 \mathrm{oms}$ & 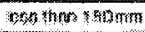 \\
\hline Wolk & 50 to 759 m & 50 le 75 mot & 30 to rom \\
\hline Hathe & Gen then Monn & 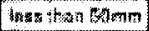 & bosthen $20 \mathrm{~nm}$ \\
\hline
\end{tabular}

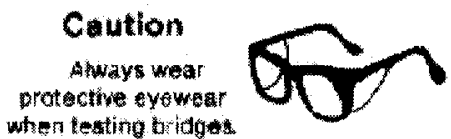

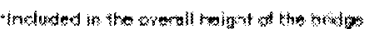

Holf: Then

4h

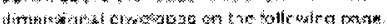

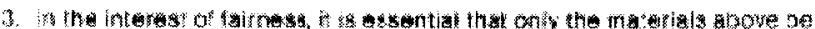

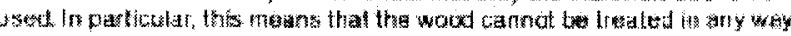

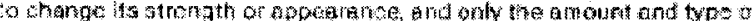

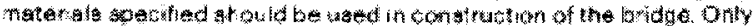

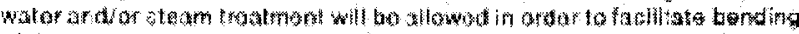

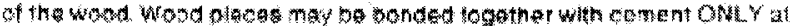

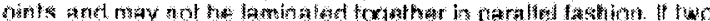

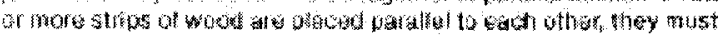

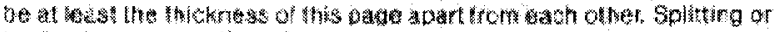

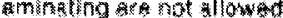
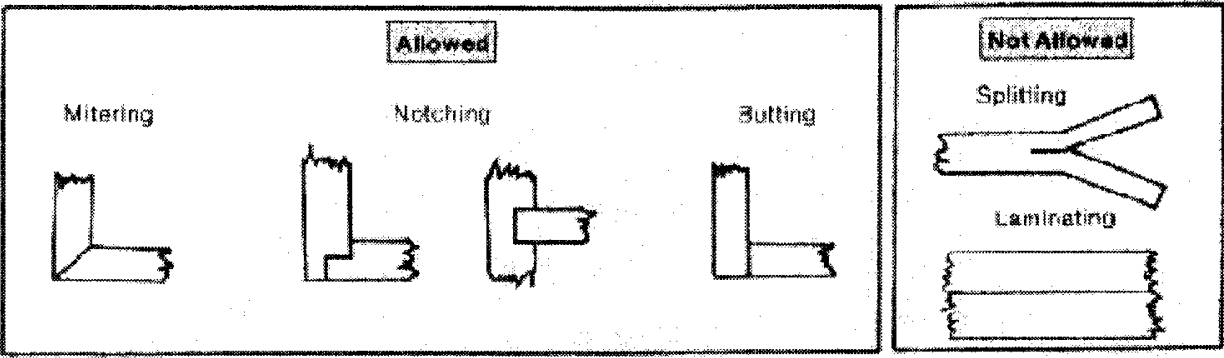

-21 MIDWEST 

Appendix G6 
SC-06-2.3.2 Students will explain cause and ef fect relationships in the Rock cyde.

Naterals found in the lithosphere ard mantle cre ckanged in a continuous probess called the rock cycle, which can be invertigated using a varlety of models. Cause and effect relationships should be explored in order to draw canclusions and nake evdence-bosed predictions of the conthuolly changing materlals boK?

Materiols
7 chocolate chips
7 white chocolate chips
7 butterscotch chips
2 knives
Woxed poper

\section{Procedure:}

\section{The Rock Cycle}

Aluminum foil 2 pairs of gloves 2 textbooks Aluminum pie plate Hot plate

1. Cover your work area with a sheet of waxed paper and place one of the types of chips on it.

2. Shave the chips into small pieces with o knife.

3. Repeat Step 1 for the other chips, making sure to keep the shavings in separate piles.

\section{Answer Question 1 , parts $A, B, \& C$ on your lab report.}

5. Fold the sheet of aluminum foil in half. Place the foll on your lab station and open it so that the crease is in the middle.

6. Sprinkle one type of chip shavings to ane side of the crense in the foil making a smail square. Pat the shavings to make an even layer and use your fingers to move the shavings in the shape of a square

7. Repeat step 6 with the other three types of shavings, making loyers of each flavor on top of the previous layer of shavings.

8. Fold the top half of the foil over the chip loyers.

9. Place the foil package between the two textbooks and apply light pressure for 2 seconds.

10. Demove the foil package from the books and open it

11. Answer Questions 2, 3, 4 in your lab repont.

12. Place the candy-chip "rock" bock in the foil and put the foil between the two books agnin.

This time, two students should press as hard os possible against the books for one minute.

13. Remove the package from between the books and open it.

14. Answer questions 5, 6. \& 7 in your lab report.

15. PUT ON YOUR GLOVES. Plug in the hol plate and turn it on.

16. Now place the aluminum pie plate on the hot plate. Then place the package with your "rock" in the pie plate and turn on the plate. Be sure the prackage is open slightly of the top so that you can observe what happens.

17. Answer questions $8 \& 9$ in your lab repart.

18. Allow your rock model to cool and harden completely.

19. Answer questions 9 and 10 in your leb report. 
Rock Cycle Lab Report

1. Identify what the following itens represent in our simulation.
A. The whole chips:
8. The knife
C. The thip stovings:

2. Oescribe what happened to the shavings

3. Tdentify what type af rack we just farmed in our simulation. Explain your answer.

4. Identify what pressing on the two textbooks represerted in the process.

5. Describe what happened to the layers of shavings this time.

6. Identify what type of rock just farmed in our simulation. Explain your answer.

7. Identify what pressing on the two lextlowes repesented in the process.

8. bescribe what is happening to the chips.

9. Identify what type of rock we just formed in cur sinulatior. Explain your answer.

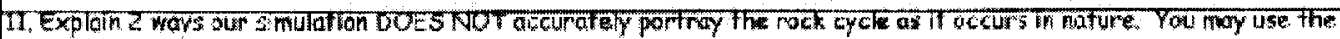
back of the page if riecessory. 


\section{CURRICULUM VITAE}

\section{Jon C. Saderholm, NBCT}

125 Goodloe Ave. Bloomfield, KY 40008

(502) 252-7817

jonsaderholm@bellsouth.net
Room 123 CEHD

University of Louisville

Louisville, KY 40292

(502) $852-2625$

jon.saderholm@louisville.edu

\section{Education}

Ph.D Curriculum and Instruction with concentration in Science Education. University of Louisville. Dissertation entitled: Science Inquiry Learning Environments Created by National Board Certified Teachers. Major Advisors: Tom Tretter and Robert Ronau. June 25, 2007

M.Ed Secondary Education with concentration in Mathematics. University of Louisville. 1999

B.A. Physics \& Mathematics with teaching certification. Berea College. 1987

\section{Current Certifications}

National Board for Professional Teaching Standards (NBPTS) Certification in Adolescent/Young (AYA) Adult Science. 1999-2009

Kentucky Provisional Teaching Certificate. Physical Science Grades 7-12.

\section{Post-Secondary Teaching Experience}

Instructor. Sue Bennett Junior College. London, KY. 1987 - 1989

I taught Physics, Physical Science and Arithmetic.

Teaching Assistant, Berea College Physics Department. 1982 - 1987

In this position I provided tutorial and lab sessions, ran astronomy observation sessions, presented planetarium shows, and ran observatory open houses among other duties.

\section{Secondary Teaching Experience}

Teacher. Spencer County High School. Taylorsville, KY. 1994 - 2004

During this time I taught Conceptual Physics, Physics, and AP Physics B, Earth Science, Geometry, Integrated Math II, Algebra II and AP Calculus. 
Teacher. Sandy Spring Friends School (SSFS). Sandy Spring, MD. 1989 - 1994

SSFS is a private Quaker boarding school north of Washington D.C. I taught Physics, Environmental Science, Earth Science, Astronomy, and Chemistry. In addition, for two years I functioned as clerk of the school's Meeting for Business-a role analogous to the chair of an institution's executive committee.

\section{Papers}

Saderholm, J.C., Ronau, R.N., Brown, E.T., \& Collins, G. (under review by the School Science and Mathematics Journal, April 2007) Validation of the Diagnostic Teacher Assessment of Mathematics and Science.

Saderholm, J.C., \& Tretter, T.R. (in press) Identification of the most critical content knowledge base for middle school science teachers.

\section{Presentations}

Tretter, T. R., Brown, S. L., Bush, W., Saderholm, J., \& Moore, B. (2007, April). Valid and reliable physical, life, and earth science content assessments for middle school teachers. Poster presented at the National Association for Research in Science Teaching (NARST) Annual Conference, New Orleans, LA.

Saderholm, J., Mitchell, N., \& Tretter, T. (2007, April). The critical thinking skills of expert teachers. Poster presented at the National Association for Research in Science Teaching (NARST) Annual Meeting, New Orleans, LA.

Tretter, T. R., Brown, S. L., Bush, W., Saderholm, J., \& Moore, B. (2007, April). Valid and reliable physical, life, and earth science content assessments for middle school teachers. Paper presented at the American Educational Research Association (AERA) Annual Meeting, Chicago, IL.

Saderholm, J.C. (2007, April) National Board Certified Teacher science inquiry learning environments. Paper presented at the American Education Research Association (AERA) Annual Meeting, Chicago, IL.

Tretter, T.R., Saderholm, J.C. \& Mitchell, N.G. (2007, January) Critical thinking skills of expert teachers. Paper presented at the Association for Science Teacher Education (ASTE) Annual Meeting, Clearwater, FL.

Saderholm, J.C. (2006, April) National Board Certification as a change agent for science teachers. Paper presented at the National Association for Research in Science Teaching (NARST) Annual Meeting, San Francisco, CA.

Tretter, T.R., Brown, S., Moore, B., Saderholm, J.C. \& Kemp, A. (2006, April) Valid and reliable physical, life, and earth science content assessments for middle school teachers. Paper presented at the National Association for Research in Science Teaching (NARST) Annual Meeting, San Francisco, CA.

Saderholm, J.C. \& Sweazy, R.A. (2006, March) National Board Certified Teachers: Leading Kentucky's teachers into the next millennium. Paper presented at the 
Kentucky Teaching and Learning Conference (KTLC), Louisville, KY.

Saderholm, J.C. \& Tretter, T.R. (2006, January) Analysis of middle school physical science content standards: What are four major standards documents really saying? Paper presented at the Association for Science Teacher Education (ASTE) Annual Meeting, Portland, OR.

Saderholm, J.C. (2005, October) National Board Certification as a change agent for science teachers. Paper presented at the Mid-Atlantic Association for Science Teacher Education Annual Meeting, Breaks Interstate Park, VA.

Saderholm, J.C. \& Tretter, T.R. (2005, October). Physical science content analysis of four influential documents: Consensus and tensions among content standards and assessment frameworks. Poster presented at the Mid-Atlantic Association for Science Teacher Education Annual Meeting, Breaks Interstate Park, VA.

Moore, B.D., Tretter, T.R., Brown, S.L. \& Saderholm, J.C. (2005, October). Development of content knowledge assessments for science teachers: Ensuring validity. Paper presented at the Mid-Atlantic Association for Science Teacher Education, Breaks Interstate Park Annual Meeting, VA.

Saderholm, J.C. \& Tretter, T.R. (2005, October) Diagnosing science teacher content knowledge. Paper presented at the Council for Post-secondary Education Teacher Quality Summit, Louisville, KY.

Saderholm, J.C., (2005, July) Creating a state association of National Board Certified Teachers. Poster presented at the National Board for Professional Teaching Standards (NBPTS) Bi-Annual Meeting, Washington D.C.

Tretter, T.R., Moore, B.D., Brown, S.L., Saderholm, J.C., Kemp, A.C. \& Bush, W.S. (2005, January) Structure and characteristics of physical science assessments designed for middle school teachers. Paper presented at the Association for the Education of Teachers of Science (ASTE) Annual Meeting, Colorado Springs, CO.

Tretter, T. R., Moore, B. D., Brown, S. L., Kemp, A. C., Saderholm, J.C. \& Bush, W. S. (2004, October) What middle school teachers need to know about physical science. Paper presented at the Mid-Atlantic Association for Science Teacher Education Annual Meeting, Roan Mountain, TN.

\section{Honors}

Completed the Future Faculty Program, University of Louisville. 2006

Recipient of the University Fellowship, University of Louisville. 2004

\section{Committee Memberships}

Emergent Technology Advisory and Research Committee. University of Louisville. 2006 - present

University of Louisville/University of Cincinnati/University of Kentucky Spring Research Conference Planning Committee. 2005 - 2006 
Academic Technology Committee, Instruction Subcommittee. University of Louisville. 2004 - present

Bloomfield Middle School Site Based Decision Making (SBDM) Council. 2001 - 2006

Spencer County High School SBDM Council. 1999 - 2004

Chair, Spencer County High School Planning and Technology Committee. 1999 - 2003

\section{Professional Roles \& Memberships}

Chair of the Board of Directors, Kentucky Association for National Board Certified Teachers (KANBCT). 2004 - 2006

Treasurer, KANBCT. 2003 - 2004

American Educational Research Association

Association for Science Teacher Education

National Science Teachers Association

American Association of Physics Teachers

\section{Other Roles}

Researcher for University of Louisville Center for Research in Mathematics and Science Teacher Development (CRMSTD) Diagnostic Teacher Assessment in Mathematics and Science (DTAMS) project. 2004 - present

In this role I wrote middle school mathematics and science teacher assessments, and performed test evaluations using structural equation modeling (SEM) and item response theory (IRT).

Reviewer for the National Association for Research in Science Teaching annual conference, New Orleans, LA. 2007

Reviewer for the American Education Research Association annual conference, Chicago, IL. 2007

Reviewer for the American Educational Research Journal. 2005 - present

Reviewer for the Association of Science Teacher Education annual conference, Portland, OR. 2006

Panelist on University of Louisville College of Education and Human Development Doctoral Program Open House. 2004

Regional Coordinator for the Kentucky Educational Professional Standards Board NBPTS Comprehensive Support System. 2004 - 2006

In this role I coordinated mentor services for the Ohio Valley Educational Cooperative (OVEC) region present along with presenting recruitment, pre-candidacy 
and leadership workshops in the northern region of the state.

Member of the Kentucky Educational Professional Standards Board NBPTS Advisory Workgroup. 2003 - 2006

Participant of the Kentucky Adequacy Study_-Western Kentucky Professional Judgment Panel Meeting. 2003

This professional judgment panel was convened by the Kentucky Department of Education to create a model for a "prototype school" to inform the department regarding the cost of providing an education that would guarantee proficiency for every student by 2014 .

Special Judge at the Intel International Science and Engineering Fair. Louisville, KY. 2002

I was on the three-member team representing the American Physical Society and the American Association of Physics Teachers that judged and awarded their special award at the International Science Fair.

National Board Certification Mentor. 2000 - 2006

During this time I worked with many NBPTS candidates at all grade levels and subject areas, attended the NBPTS "Facilitator 1" training, and worked with the OVEC NBPTS Collaborative.

School Technology Coordinator and Student Technology Leadership Program (STLP) coordinator. Spencer County High School. 1999 - 2004

In this role I supervised and maintained a network of in excess of 200 Windows 2000 computers. This role included supervision of student workers, creation and implementation of Internet access policy, and creation of long-term plans.

Southern Association of Colleges and Schools (SACS) School Improvement Plan (SIP) Peer Review team member for Owen County High School, Owen County Kentucky. 2002

SACS SIP Peer Review team member for Doss High School, Jefferson County Kentucky. 2001

Initiation and coordination of the study regarding, and subsequent change from standard schedule to block schedule at Spencer County High School. 1998 - 1999

Clerk of the Sandy Spring Friends School Meeting for Business. 1992 - 1994

Sandy Spring Friends School is a Quaker Boarding School and as such is governed by a Quaker Meeting for Business. The clerk of a Meeting for Business is analogous to the chairperson of a committee. 